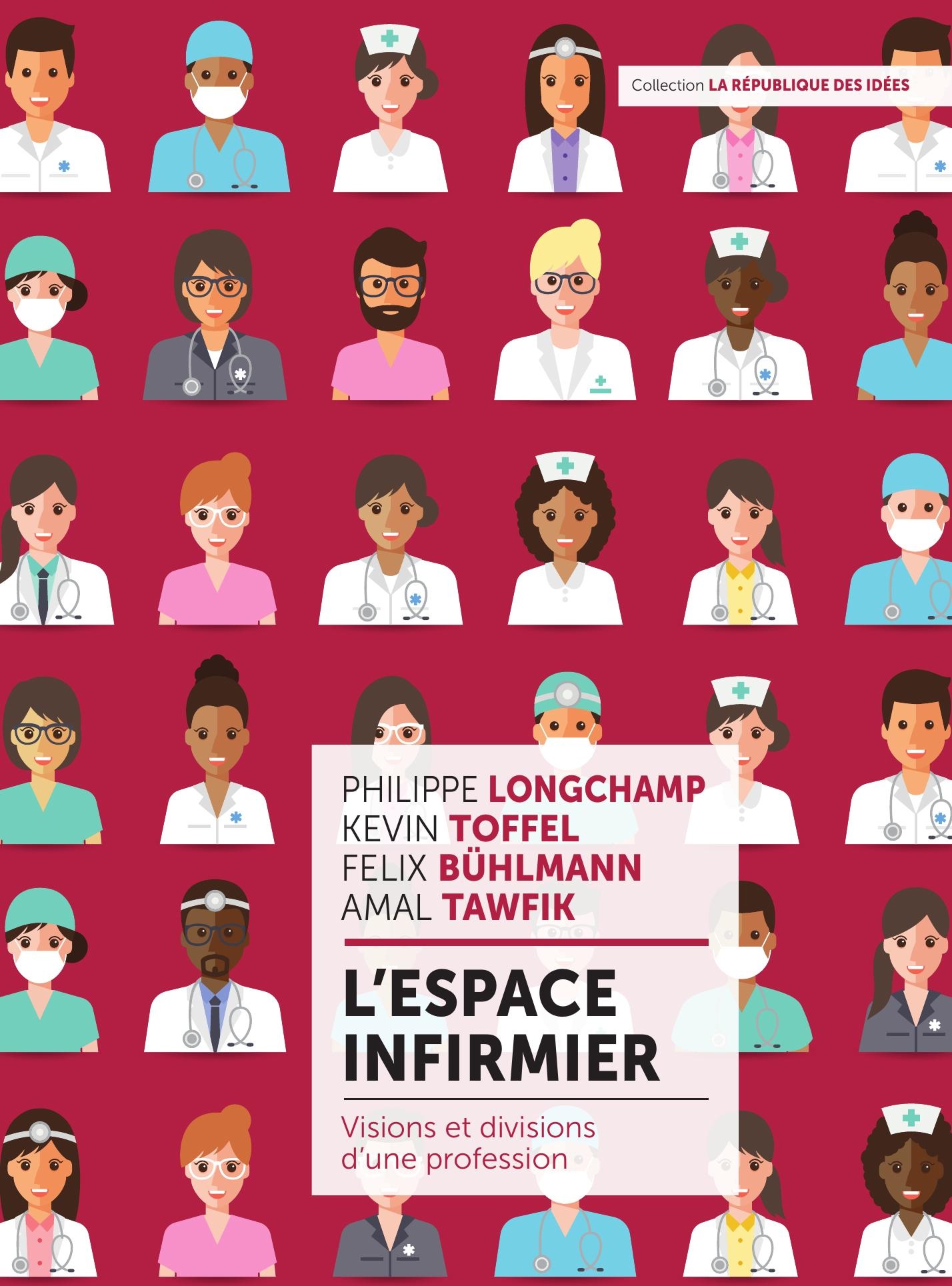





\section{L'ESPACE INFIRMIER}

VISIONS ET DIVISIONS D'UNE PROFESSION 

Philippe Longchamp, Kevin Toffel, Felix Bühlmann et Amal Tawfik

\section{L'ESPACE INFIRMIER}

VISIONS ET DIVISIONS D'UNE PROFESSION

ÉDITIONS LIVREO-ALPHIL 
(C) Éditions Livreo-Alphil, 2020

Case postale 5

2002 Neuchâtel 2

Suisse

www.alphil.ch

Alphil Diffusion

commande@alphil.ch

ISBN papier: 978-2-88950-051-2

ISBN PDF : 978-2-88950-052-9

ISBN EPUB : 978-2-88950-053-6

DOI : 10.33055/ALPHIL.01517

Publié avec le soutien du Fonds national suisse de la recherche scientifique.

Les Éditions Alphil bénéficient d'un soutien structurel de l'Office fédéral de la culture pour les années 2016-2020.

Illustration de couverture: composée à partir d'une image issue de Shutterstock

Couverture, maquette et réalisation: Nusbaumer-graphistes sàrl, www.nusbaumer.ch

Ce livre est sous licence:

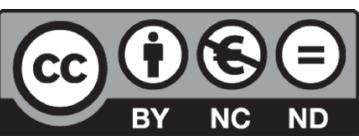

Ce texte est sous licence Creative Commons: elle vous oblige, si vous utilisez cet écrit, à en citer l'auteur, la source et l'éditeur original, sans modifications du texte ou de l'extrait et sans utilisation commerciale.

Responsables d'édition: Anne-Caroline Le Coultre, Marie Manzoni 

Le réel n'est jamais "ce qu'on pourrait croire» mais il est toujours ce qu'on aurait dîu penser.

Gaston Bachelard 


\section{Introduction ${ }^{1}$}

À en juger par leur nombre, les infirmières ${ }^{2}$ forment le pilier des systèmes de santé des pays occidentaux. Avec environ neuf infirmières pour 1000 habitants, les pays de l'OCDE comptent ainsi près de trois fois plus d'infirmières que de médecins (OCDE, 2017) ${ }^{3}$. Si la croissance continue des effectifs infirmiers depuis 1960 est étroitement liée à celle du secteur public (Euzinga, 1990), elle s'explique aussi par le vieillissement de la population qui entraîne une rapide progression de l'emploi dans le système de santé (OFS, 2007) .

Cet accroissement des effectifs s'accompagne d'un double mouvement de différenciation de la profession: horizontal d'une part, avec la multiplication des secteurs extrahospitaliers; vertical d'autre part, avec la mise en place d'une hiérarchie

\footnotetext{
${ }^{1}$ Les résultats présentés dans cet ouvrage ont pour partie été publiés sous différentes formes. Voir Longchamp \& Toffel (2016), Longchamp, Toffel, Bühlmann \& Tawfik (2016), Toffel \& Longchamp (2017), Longchamp, Toffel, Bühlmann \& Tawfik (2018), Toffel (2018), Toffel (2020).

${ }^{2}$ Afin d'alléger la formulation, et en tenant compte de la nette majorité féminine de la profession, le mot «infirmière» désigne également le masculin dans l'ensemble du texte.

${ }^{3}$ OCDE: Organisation de coopération et de développement économiques.

${ }^{4}$ OFS: Office fédéral de la statistique.
} 
soignante propre (FEroni \& Kober-Smith, 2005) et le processus d'académisation des formations aboutissant à de nouvelles spécialisations, tant dans le domaine clinique que dans celui de la recherche. Ce double mouvement de différenciation est particulièrement marqué en Suisse romande. Les six écoles de soins infirmiers ${ }^{5}$ qui y délivrent depuis 2002 un diplôme de niveau bachelor sont le fruit d'un long processus historique de laïcisation et d'académisation (Droux, 2000). Cette évolution s'est poursuivie avec l'ouverture, en 2009, de filières master et doctorat à l'Université de Lausanne 6 . À ces changements rapides qui touchent la formation s'ajoute un très fort mouvement d'externalisation de certaines prises en charge jusqu'ici assurées par l'hôpital, notamment en ce qui concerne le vieillissement et les maladies chroniques (Jaccard Ruedin, Weaver, Roth \& Widmer, 2009), ce qui tend à renforcer le processus de différenciation de la profession avec une multiplication du nombre de postes extrahospitaliers. Au total, la différenciation de la profession et le développement des activités de recherche par les infirmières sont ainsi plus marqués en Suisse romande que dans d'autres pays qui, comme la France, connaissent un développement académique de la formation moins avancé 7 .

\footnotetext{
${ }^{5}$ Haute École de Santé Vaud; Haute École de Santé La Source; Haute École de Santé Fribourg; Haute École de Santé Arc; Haute École de Santé Genève; Haute École de Santé Valais.

${ }^{6}$ L'académisation de la formation en soins infirmiers a été beaucoup plus précoce dans d'autres pays. L'Amérique du Nord a été pionnière, avec l'introduction d'une formation en soins à l'université dès 1907 aux ÉtatsUnis (Teachers College de l'Université de Columbia) et en 1919 au Canada (Université de Colombie-Britannique). L'Europe a suivi un demi-siècle plus tard, au Royaume-Uni d'abord (ouverture d'un diplôme de soins infirmiers en 1959 puis d'un bachelor en 1969 à l'Université de Manchester), en Europe du Nord ensuite (intégration des soins à l'université en 1977 en Suède) (Chapman, 1982; Elzinga, 1990; McPherson, 2003).

${ }^{7}$ Isabelle Feroni et Anémone Kober (1995) mentionnent, pour la France, la faiblesse des liens entre les instituts de formation en soins infirmiers et les milieux académiques.
} 
Ce double mouvement de différenciation entraîne une multiplication des différentes positions repérables au sein de la profession et, partant, est susceptible d'augmenter les tensions liées à l'identité soignante, tiraillée entre les trois rôles que sont la spécialisation/technique, la polyvalence/relation et la coordination/interprofessionnalité. On sait en effet que cette tension, qui peut engendrer un «malaise structurel» au niveau individuel (Dubet, 2002) ou des tensions entre soignantes au niveau des collectifs de travail (Acker, 1995; Feroni \& Kober, 1995; VÉGA, 1997), peut aussi s'incarner dans les différentes positions repérables au sein de la profession, avec notamment les oppositions entre secteurs hospitaliers et extrahospitaliers (De Montlibert, 1980), «lents» et «rapides» (Douguet \& Vilbrod, 2007) ou encore féminins et masculins (Picot, 2005). L'analyse de la variation des définitions de la pratique soignante en fonction des différentes positions occupées s'avère d'autant plus importante que ces positions sont en voie de multiplication et de distanciation les unes par rapport aux autres. C'est donc à la question générale de la relation entre les positions qu'occupent les infirmières au sein d'une profession hautement différenciée d'une part, et leurs prises de position (représentations et pratiques) du métier d'autre part, qu'entend répondre cette recherche. Bien qu'elle soit évoquée dans certains travaux portant sur la profession, cette question n'a pas encore fait l'objet d'une analyse systématique à notre connaissance.

La recherche d'une correspondance entre positions et prises de position implique une double exigence, théorique et méthodologique. Sur le plan théorique, elle nécessite un cadre conceptuel capable non seulement d'embrasser l'ensemble des secteurs de la profession infirmière, mais aussi d'en restituer les enjeux. Parce qu'elle permet de saisir de manière simultanée l'ensemble des positions d'une profession donnée, la théorie des champs de Pierre Bourdieu nous semble des plus pertinentes. En insistant sur les rapports sociaux qui relient et opposent les occupants des différentes positions, elle permet en effet de 
penser ensemble l'unité et la diversité, et ainsi d'analyser les relations structurales qui, tout en intégrant les interactions de face à face, règlent aussi les rapports qu'entretiennent des individus déterminés par les positions objectives qu'ils occupent les uns relativement aux autres au sein de la profession.

Sur le plan méthodologique, notre approche structurale exige d'analyser l'ensemble des positions repérables au sein de la profession à l'échelle d'une aire géographique donnée. C'est ce que nous avons réalisé en menant des entretiens avec des infirmières exerçant en Suisse romande, puis en distribuant un questionnaire à un échantillon représentatif de cette population. Les résultats montrent que la profession infirmière est segmentée en un ensemble de positions auxquelles sont associées des représentations et pratiques spécifiques s'agissant de la définition légitime des soins, de la nécessité de développer un savoir propre ou encore du positionnement à adopter face aux médecins.

Ce livre s'adresse aussi bien aux sociologues, aux infirmières, aux étudiantes et étudiants en soins qu'aux responsables politiques. Les lecteurs sociologues trouveront une originalité dans l'approche théorique, qui consiste à mobiliser la théorie des champs pour l'analyse d'un espace professionnel fortement féminisé et traditionnellement dominé. Cette option ne va pas de soi, notamment parce que, en tant que théorie du pouvoir, la théorie des champs est habituellement mobilisée pour l'étude de microcosmes fortement masculinisés situés dans les régions supérieures de l'espace social (LaHiRe, 2001). Les infirmières y trouveront une description fine des différentes facettes de leur métier ainsi qu'une approche alternative aux représentations souvent homogénéisantes de la profession. En mettant en évidence non seulement la diversité, mais aussi les inégalités qui la caractérisent, notre perspective permet de mieux identifier les enjeux actuels de la profession sur la base desquels des stratégies professionnelles visant davantage d'autonomie et de reconnaissance pourraient être formulées. Les étudiantes et étudiants en soins trouveront dans ce livre une véritable 
"photographie» de la profession qui leur permettra d'effectuer des choix de carrière mieux renseignés. Au-delà des informations relatives aux différents secteurs d'activité, la description et l'analyse des enjeux et des mécanismes de pouvoir leur permettront de développer un regard à la fois critique et réflexif sur leur future pratique. Quant aux responsables politiques, ils trouveront dans ce livre tant des données factuelles inédites sur la profession, que matière à réflexion concernant son avenir.

L'ouvrage est organisé autour d'une analyse de la structure de ce que nous appelons l' «espace infirmier», présenté dans la première partie. La description de cet espace est précédée d'un bref historique qui montre comment la profession, après avoir connu une domination religieuse jusqu'à la fin du XIX $^{\mathrm{e}}$ siècle, puis une domination médicale durant le $\mathrm{xx}^{\mathrm{e}}$ siècle, cherche aujourd'hui à s'émanciper notamment par le développement d'une discipline scientifique spécifique - les sciences infirmières - qui propose des connaissances produites par et pour les infirmières. Dans la deuxième partie, nous identifions quatre fractions d'infirmières: les "dominantes médicales», les "dominées médicales", les «élites infirmières» et les "hétérodoxes». Occupant des positions distinctes au sein de la profession, les infirmières de ces quatre fractions se caractérisent par des conceptions spécifiques de leur pratique. Dans la troisième partie enfin, nous proposons d'analyser les valeurs fondatrices de la profession, telles que les motivations de type humaniste ou encore l'opposition entre les dimensions «technique» et "relationnelle» des soins. Formant un véritable "ethos infirmier", ces valeurs sont analysées en regard de la diversité de la profession mise en évidence dans les deux premières parties. 



\section{Partie I}

\section{Une profession homogène?}

Alors que la profession infirmière jouit d'une forte visibilité dans l'espace public, elle demeure paradoxalement très mal connue; elle est par ailleurs perçue dans l'imaginaire collectif selon des catégories qui l'homogénéisent. À cette représentation relativement stable et uniforme de la profession sont associées des situations et des pratiques: elle serait composée de femmes le plus souvent vêtues de blanc et secondant les médecins dans la prise en charge de personnes en situation de maladie ou d'accident, généralement en milieu hospitalier. Une telle vision remplit aussi une fonction pour les porte-parole de la profession qui peuvent ainsi parler au nom d'un groupe fédéré, comme on a pu le constater en 2017 lors de la récolte de signatures dans le cadre du lancement de l'initiative populaire «Pour des soins infirmiers forts» ${ }^{8}$.

\footnotetext{
${ }^{8}$ Lancée par l'Association Suisse des Infirmières (ASI), cette initiative vise une revalorisation et une plus grande reconnaissance de la profession, notamment en définissant certains soins pouvant être directement pris en charge par l'assurance maladie sans être prescrits par les médecins. Celle-ci a été rejetée par le Conseil fédéral le 9 mars 2018. Le 24 janvier 2019, le Conseil national a proposé un contre-projet indirect intitulé "Pour un renforcement des soins infirmiers, une sécurité des patients accrue et une meilleure qualité des soins». La Commission de la sécurité sociale et de la
} 
Bien que reposant sur des éléments socialement et légalement fondés, cette vision ne permet de saisir ni la complexité sous-jacente à cette attribution ni les enjeux associés à cette vision homogène. Il revient précisément à l'analyse sociologique d'interroger ce que masque cette image unifiée. Dans cette première partie, nous montrons que considérer la profession infirmière comme un espace de luttes entre des individus occupant des positions spécifiques permet de saisir les enjeux propres à la structuration de la profession, enjeux qui sont étroitement liés à un développement historique marqué par les dominations.

santé publique du Conseil des États (CSSS-E) ayant donné son accord à cette initiative parlementaire, cette Commission travaille depuis à l'élaboration d'un contre-projet de loi. 


\section{Chapitre 1 \\ Éléments d'histoire de la profession infirmière ${ }^{9}$}

La compréhension des enjeux aujourd'hui spécifiques à la profession infirmière nécessite une rapide description des développements successifs qui l'ont caractérisée par le passé. Nous montrons dans ce chapitre que la question du genre est centrale dans la façon dont se sont construits les rapports qu'entretient cette profession féminine avec les instances religieuses d'abord, médicales ensuite. Cette lecture nous permet d'aborder le long processus de professionnalisation de la profession infirmière, jusqu'à en arriver à une discipline infirmière participant aujourd'hui pleinement de sa (re)définition.

\section{De la profession de foi à la profession}

En suivant Marie-Françoise Collière, on peut dire que «tout le dilemme de la profession infirmière repose [...] sur l'exigence de passer d'une profession de foi fondée sur un "credo" ayant pour finalité de servir, d'être au service de (des malades dans les temps plus anciens,

${ }^{9}$ Le contenu de ce chapitre est largement issu de Toffel (2018). 
des médecins par la suite), à une nécessaire clarification du service offert» (Collière, 1982, p. 235, souligné par l'auteure). C'est de cette façon qu'elle percevait la situation au début des années 1980. Près de 40 ans après, force est de constater que ce dilemme est encore largement perceptible, et ce malgré les nombreuses transformations que la profession a connues depuis. Les ressorts sous-jacents à cette aporie demeurent en effet présents et forment l'un des socles identitaires sur lequel s'est construite la profession.

On peut repérer au moins trois aspects qui sont au fondement du dilemme qu'évoque Collière et qui concourent historiquement à façonner l'établissement de la profession. Premièrement, pour comprendre la position qu'occupent les infirmières aujourd'hui dans le système de soins ainsi que leurs prises de position relatives aux conditions et au contenu de leur travail, il faut prendre en compte le caractère éminemment féminin de cette profession. Deuxièmement, il faut considérer son héritage religieux. Bien que l'on se doive de prendre quelque distance avec les représentations sociales encore communément véhiculées d'une profession infirmière perçue comme l'héritière de la vocation religieuse, il n'en reste pas moins que cet héritage a durablement marqué la profession - en particulier dans les représentations qui drapent la dimension «relationnelle» des soins. Enfin, il faut aborder le passage de la subordination des "soignantes religieuses», fondée sur une morale ecclésiale dont la dévotion aux malades était la clé, à la subordination des professionnelles des soins dans une logique essentiellement médicale, où l'allégeance est alors avant tout faite aux médecins. Ce passage de la domination de l'Église à celle du champ médical marque structurellement le processus de professionnalisation de la profession.

\section{Le mythe de la «femme soignante »}

L'origine du rôle assigné aux «femmes soignantes» (Collière, 1982) repose sur une division sexuelle du travail qui remonte très 
loin dans l'histoire du soin. À cette division du travail du point de vue de ceux qui l'accomplissent, s'ajoute la différenciation de ceux à qui ces soins sont prodigués. Comme le remarque André Petitat (1989), les soins que l'on peut qualifier de "curatifs» ont traditionnellement été pris en charge par la figure masculine $\mathrm{du}$ "garde malade», figure qui a pris des allures différentes au fil de l'histoire: "bandagistes»; "apprentis médecins»; «soldats» (p. 41-42). Ces soins, donnés dans des contextes spécifiques (la plupart du temps guerriers et militaires, ou plus communément lors d'accidents de chasse), et prodigués par des hommes à d'autres hommes, ne doivent pas être confondus avec le rôle de «la femme qui aide» (Collière, 1982, p. 16). Alors que la tâche des hommes relève strictement de soins d'urgence ponctuels, celle des femmes consiste essentiellement à dispenser des "[...] soins courants, c'està-dire tous les soins qui entretiennent la vie de tous les jours [...]" (p. 38); un type de soins prodigués aux femmes et aux nouveau-nés, aux enfants et aux mourants - autrement dit, aux populations «vulnérables»-que l'on qualifierait aujourd'hui de $\operatorname{care}^{10}$.

Cette «condition de femme» renvoyée à ses qualités «morales» lorsqu'il s'agit d'une religieuse, ou à ses aptitudes «naturellement maternelles», ne sera pas totalement remise en question par la professionnalisation qui apparaît avec le «tournant laïque» au XIx ${ }^{\mathrm{e}}$ siècle, loin s'en faut.

\section{Au service de l'Église}

Joëlle Droux (2000) note que «le premier (et le plus durable) des socles identitaires sur lesquels la profession s'est historiquement constituée, en Suisse comme ailleurs, c'est assurément le socle de

${ }^{10}$ Quoique polysémique, la notion de care renvoie généralement à des pratiques dont les compétences, notamment basées sur le prendre soin, l'empathie, l'écoute, la sensibilité, etc., reposent sur une "construction idéologique selon laquelle les femmes, [étant] considérées comme plus affectives que les hommes, [...] ont plus d'aptitudes pour le soin" (TRONTO, 2008: 255). 
la vocation religieuse» (p. 30). Cette identité est cependant relativement récente; tout au plus remonte-t-elle au Moyen Âge alors que, nous venons de le voir, le rôle et l'inscription des femmes dans la dispensation des soins sont bien plus anciens.

Actives dans les soins depuis toujours, les "soignantes" ont accumulé un grand nombre de connaissances et se sont transmis différents savoirs et savoir-faire (Collière, 1982). Mais alors qu'au Moyen Âge l'Église gagne considérablement de pouvoir en Europe, une lutte s'instaure au sujet de ces «femmes soignantes" qui sont considérées comme une menace pour l'ordre social que défend âprement cette puissante institution. S'ensuit une chasse aux sorcières qui participe d'un mouvement général de "réprobation des cultes païens" au tournant de la Renaissance. Parallèlement, la profession médicale qui voit le jour de façon institutionnalisée avec l'apparition d'un enseignement universitaire dès le XIII siècle en Europe débute lentement son processus d'émancipation vis-à-vis de l'Église. La médecine naissante se débarrasse des savoirs et savoir-faire hérités de la pratique des «femmes soignantes» - qui ont de surcroît développé de nombreux remèdes à l'efficacité éprouvée - et considère ceux-ci comme relevant de la sorcellerie (Ehrenreich \& English, 1976). Selon Barbara Ehrenreich et Deirdre English, c'est une «lutte doublement politique» qui se déroule donc depuis le Moyen Âge: une lutte des sexes où les hommes tendent à conserver le monopole sur le soigné, et une lutte des classes où les sciences médicales émergentes enseignées à l'élite d'alors s'opposent aux savoirs populaires largement transmis par les femmes soignantes. Attaquées par l'Église - dont elles ne dépendent pas - et remises en cause par l'apparition de la profession médicale, les «femmes soignantes» vont progressivement disparaître de la pratique légitime de soins.

C'est dans ce contexte que les religieuses prennent une place grandissante dans les soins, en particulier dans le cadre hospitalier. Car si les nonnes étaient bien présentes dans les 
hôpitaux dès le Haut Moyen Âge (Laget \& SAlaün, 2004), la transformation de cette institution va modifier leur rôle. On passe progressivement, au sortir du Moyen Âge, d'un "hôpital chrétien [qui] est pensé et organisé comme un lieu d'accueil plus que comme un centre de soins [...]", et dans lequel la prise en charge des miséreux est une tâche qui revient comme "naturellement" aux religieuses, à "l'institution de la clinique, à savoir le développement de pratiques d'observation et d'enseignement au lit du malade» (LAGET \& SAlaün, 2004, p. 21-24).

Plusieurs caractéristiques, telles que l'«éternel féminin», le «bénévolat» ou encore la "tâche missionnaire» concourent à l'établissement durable des sœurs dans cette sphère des soins (Droux, 2000). Cependant, et contrairement aux «femmes soignantes" évoquées jusqu'ici, l'essentiel de la prise en charge religieuse ne repose pas vraiment sur les soins aux corps jusqu'à la fin du XIX ${ }^{\mathrm{e}}$ siècle. Leur rôle est essentiellement moral et repose sur l'ascétisme, la charité et le don de soi qui se sont développés dans les communautés chrétiennes durant le Moyen Âge.

\section{L'avènement d'une professionnelle des soins}

On peut considérer que la transition de la «soignante religieuse" à l'infirmière professionnelle des soins s'effectue à l'orée du XIx ${ }^{\mathrm{e}}$ siècle, d'une part avec le mouvement de laïcisation des hôpitaux au cours du XviII ${ }^{\mathrm{e}}$ siècle, et d'autre part avec l'émergence du "champ de la médecine moderne» (Pinell, 2009). Ces deux mouvements - laïcisation et médicalisation - sont intimement liés et leurs effets sur la profession infirmière sont solidaires, en particulier sur les plans de sa professionnalisation et de son inscription au sein d'un champ médical dont la structure se solidifie au cours du $\mathrm{XIX}^{\mathrm{e}}$ siècle. 


\section{Un processus de laïcisation en demi-teinte}

Alors que l'un des mythes fondateurs de la profession repose sur la figure de la religieuse, il est intéressant de noter que, paradoxalement, c'est "la lä̈cisation des soins [qui] scelle [...] l'acte de naissance de la profession infirmière» (Knibiehler, 2008, p. 57). En fait, ceci n'est contradictoire qu'en apparence.

Largement influencée à la fois par l'évolution des techniques et des savoirs médicaux au XIX ${ }^{\mathrm{e}}$ siècle et par la transformation du rôle de l'hôpital, l'expansion rapide des savoirs médicaux ainsi que le processus de spécialisation du champ médical qui en découle (Pinell, 2005) accélèrent le développement des hôpitaux dans leur acception moderne. L'hôpital, qui avait jusqu'alors été dédié aux indigents et aux populations marginales, devient un "centre d'enseignement et de recherche»; c'est alors le lieu où «tout se passe»(Petitat, 1989). En lien avec l'avènement des assurances sociales ${ }^{11}$, la vocation des infirmières, et notamment sa dimension de charité chrétienne, s'amenuise petit à petit: "La vocation se meurt, la profession s'épanouit» (РетітAт, 1989, p. 59).

Si la professionnelle laïque finira par s'imposer, la dimension religieuse - repérable à l'idée de vocation - va, dès le tournant $\mathrm{du} \mathrm{Xx}^{\mathrm{e}}$ siècle, perdurer sous différents visages, notamment dans la dimension «relationnelle» des soins.

Le mouvement de laïcisation qui se déroule au cours du XIX ${ }^{\mathrm{e}}$ et durant la première moitié $\mathrm{du} \mathrm{Xx}^{\mathrm{e}}$ siècle est un long processus, durant lequel le pouvoir des religieuses au sein des institutions hospitalières ne décline que très lentement. Plusieurs raisons concourent à cela. Tout d'abord, la médicalisation de l'espace de santé requiert toujours plus de connaissances de la part des

\footnotetext{
${ }^{11}$ Pour une histoire comparée des assurances sociales en Europe, voir DREYFus (2009).
} 
infirmières pour accompagner les médecins dans la prise en charge des patients. Mesurant l'opportunité que représente l'hôpital pour entrer en contact avec les familles, les institutions religieuses encouragent les sœurs à se former, ces dernières devenant alors "parfaitement compétitives» (Petitat, 1989, p. 237) face aux laïques.

C'est donc au travers de la formation que la professionnalisation des infirmières se réalisera. À une tradition pratique et «artisanale» qu'elles apprenaient au lit du malade se substitue de plus en plus un apprentissage théorique enseigné dans des écoles d'infirmières (Petitat, 1989). Cette formation a une conséquence importante pour les représentations et pratiques d'un métier en pleine mutation: "Le soin de qualité est d'abord un soin techniquement irréprochable; les dispositions religieuses ou humanitaires passent au second plan" (Petitat, 1989, p. 46).

Pourtant, et bien qu'elles soient laïques, les écoles privées perpétuent une conception très conservatrice et peu propice à l'émancipation des infirmières. L'analyse menée par Droux (2000) sur l'école du Bon Secours à Genève est à ce titre tout à fait éclairante: "Basé sur une vision très orthodoxe du rôle de la fermme dans la société, le Bon Secours ne chercha pas à initier celle-ci à une nouvelle profession dotée d'un meilleur statut social et de perspectives matérielles attractives et épanouissantes: tout au contraire, l'éthique professionnelle inculquée aux élèves vise à réaffirmer l'ancrage caritatif et l'identité spirituelle d'une vocation dévorante impliquant de leur part le sacrifice de leur vie à une cause supérieure et transcendante» (p. 290).

Le passage de flambeau des «soignantes religieuses» aux infirmières professionnelles n'est donc pas simple. Bien que la formation se développe au sein d'écoles laïques, et que la pratique d'infirmière se professionnalise, cela ne veut pas dire que celle-ci s'autonomise, bien au contraire. Et comme on va le voir, ce processus n'est pas davantage synonyme d'autodétermination. 


\section{De la domination religieuse à la domination médicale}

Parallèlement au mouvement de laïcisation, un autre facteur est à prendre en compte: celui des transformations du champ médical qui, suite à l'avènement d'une médecine scientifique à la fin du $\mathrm{XIX}^{\mathrm{e}}$ siècle, tend à se techniciser et à se différencier (Faure, 1994; Pinell, 2009). Les médecins délèguent alors de plus en plus fréquemment des tâches aux infirmières, sur le modèle d'une "nouvelle division du travail» (SENOTIER, 1992). Les infirmières doivent être de mieux en mieux formées - et notamment dans des écoles bien souvent fondées et dirigées par des médecins (Droux, 2000; Senotier, 1992).

Alors que la profession naissante n'avait pour ainsi dire pas d'organisation indépendante jusqu'au début $\mathrm{du} \mathrm{xx}^{\mathrm{e}}$ siècle, la laïcisation, loin d'apporter une autonomie aux infirmières, les enferme sous une nouvelle tutelle. Bien sûr, les activités de soins se sont quelque peu spécialisées durant le $\mathrm{XIX}^{\mathrm{e}}$ siècle, et certaines tâches liées à des prises en charge médicales spécifiques donnent à des infirmières (que l'on dirait aujourd'hui "spécialisées») des compétences spécifiques. Mais tout se passe comme si la profession avait simplement changé de maître. C'est ainsi que l'on peut dire avec Marie-Françoise Collière (1982) que les infirmières sont aujourd'hui à la fois les "héritière $[\mathrm{s}] d u$ modèle religieux et [qu'elles sont] soumise[s] au modèle médical» (p. 85).

\section{L'avènement d'une discipline infirmière: un pas de plus vers l'autonomie de la profession?}

L'académisation de la profession, tardive en Suisse, commence outre-Atlantique, où les premières formations universitaires débutent au tournant $\mathrm{du} \mathrm{xx}^{\mathrm{e}}$ siècle (LAMBERT \& LAMBERT, 2005). Aux États-Unis, les premières recherches infirmières apparaissent ainsi dans les années 1920. Il faudra 
cependant attendre le début des années 1960 pour voir se former un langage spécifique aux soins infirmiers, allant de pair avec le développement et l'élaboration d'un savoir propre qui participe d'une affirmation disciplinaire. Les premières tentatives de théorisation et de formalisation d'une pensée spécifiquement infirmière seront portées dès ces années par des infirmières au profil académique telles que Hildgard Peplau ou Virginia Henderson (Gortner, 2000).

Les années 1980 marquent le passage d'une recherche infirmière, à savoir la conduite d'opérations de recherche et la mise en place de protocoles menés par des infirmières, à une véritable science infirmière, à savoir la constitution de recherches qui répondent à des questions et des hypothèses basées sur des cadres théoriques et des finalités infirmières (GORTNER, 2000). Issue des sciences humaines et destinée à élaborer un savoir sur le corps humain et son rapport à la maladie, la science infirmière a notamment pour objectif de proposer des modèles de soins.

Force est cependant de constater que les débats restent vifs jusqu'à ce jour sur la question de la définition d'une discipline infirmière; débats concernant tant la définition d'une (ou des) science(s) infirmière(s) que des rapports qu'entretiendrait la discipline infirmière à la profession infirmière. Alors que Afaf I. Meleis (1991) avance que l'une des caractéristiques de la discipline des sciences infirmières est de renforcer le pouvoir des infirmières (to empower nurses), Sue K. Donaldson (2003a) insiste au contraire sur une distinction nette qu'il s'agit d'établir entre la science infirmière et la profession infirmière: deux entités qui, selon elle, seraient liées mais distinctes. Si l'on peut penser que la profession s'appuie bien, dans la pratique des soins, sur les savoirs formulés par la discipline, les objectifs de ces deux entités diffèrent - l'un renvoyant aux enjeux d'une profession, l'autre à ceux d'une discipline scientifique - et se doivent selon Donaldson d'être distingués. Pour parfaire cette distinction, la chercheuse propose même d'oublier le terme de «science infirmière» (nursing science) pour lui substituer celui 
de «science de la santé humaine» (science of human health). En effet, selon elle, la question centrale est celle de développer des savoirs sur la santé humaine, et non de viser une «élévation $d u$ statut des soins infirmiers» (2003b, p. 180, traduit par nous). L'identité mais aussi l'objet des sciences infirmières demeurent ainsi à l'état de questions ouvertes.

On le voit, les controverses au sein de la discipline infirmière sont loin d'être dénouées. Il est sans doute encore trop tôt pour évaluer les effets de l'apparition de ces savoirs propres, et notamment pour juger s'ils peuvent réellement contribuer à l'émancipation de la profession. En tous les cas, l'apparition d'une discipline infirmière constitue sans doute le dernier événement marquant dans le développement de la profession. Aussi, et alors que Marie-Françoise Collière (1982) voyait juste lorsqu'elle parlait au début des années 1980 d'une polarisation de la fonction de "femme soignante" entre un «rôle moral» et un «rôle technique» (p. 16), cette polarisation tend aujourd'hui à se complexifier avec l'apparition d'une professionnelle des soins doublée d'une chercheuse entreprenant d'élaborer un arsenal théorique au service de la profession. Et comme nous le verrons, cette pluralisation des profils infirmiers contribue directement à la constitution d'un espace professionnel différencié. 


\section{Chapitre 2 \\ Quelques éléments descriptifs de la profession}

En raison de l'absence d'un registre statistique national dédié aux infirmières, les connaissances relatives à la composition de ce groupe professionnel demeurent limitées en Suisse. Les données récoltées dans le cadre de notre projet auprès d'un échantillon représentatif des infirmières de Suisse romande ( 2923 individus $)^{12}$ viennent partiellement pallier ce manque de connaissances. Avant d'entrer dans le vif de l'analyse sociologique, ce chapitre vise à livrer un bref aperçu de la composition de notre échantillon en termes de genre, d'âge, de formation, de nationalité, de revenu et d'origine sociale.

\section{Genre, âge et formation}

Nos données confirment le caractère très féminisé de la profession (voir tableau A1 en annexe 3): la part des hommes de notre échantillon s'élève à seulement $15,7 \%$, soit un taux un peu plus élevé que celui que l'on observe à l'échelle nationale, évalué à $11 \%$ (Jaccard Ruedin, Roth, Weaver \& Widmer, 2009). Ce

${ }_{12}$ Pour une description précise de la constitution de l'échantillon, voir annexe 1. 
sexe-ratio subit d'importantes variations en fonction des différents secteurs d'activité. Alors que certains - tels l'obstétrique, le pool de remplacement, la santé scolaire, l'oncologie, la gynécologie, la pédiatrie, les soins à domicile, l'entreprise et l'équipe mobile - sont composés quasiment exclusivement de femmes, d'autres - comme la psychiatrie, le bloc opératoire et les urgences - sont quant à eux composés d'un quart à presque un tiers d'hommes. Le secteur de l'anesthésie est quant à lui le seul à présenter une majorité d'hommes (58,8\%). Ceux-ci sont également surreprésentés dans les fonctions hiérarchiques, qu'il s'agisse des postes de cadre $(25,9 \%)$ ou de direction (31,8\%). La différence des taux d'activité n'est probablement pas étrangère à ces inégalités, les hommes affichant un taux moyen supérieur (92,6\%) à celui des femmes (78,1\%).

L'âge moyen est de 43 ans (voir tableau A2 en annexe 3). Un tiers des infirmières sont âgées de 50 ans et plus ${ }^{13}$. Ici encore, on enregistre d'importantes variations selon les secteurs d'activité. Alors que les infirmières exerçant dans des secteurs hospitaliers tels que les soins intensifs, les urgences, la pédiatrie et la médecine sont âgées de moins de 40 ans en moyenne, celles qui exercent dans des secteurs extrahospitaliers tels que les soins à domicile, les établissements médicaux sociaux (EMS) ${ }^{14}$, la santé scolaire et les établissements socio-éducatifs ont plus de 45 ans en moyenne.

Plus de $80 \%$ des infirmières ont suivi une formation complémentaire après leur formation de base ${ }^{15}$. Si certaines

\footnotetext{
${ }^{13}$ Dans son enquête à l'échelle nationale, l'Observatoire suisse de la santé (Jaccard Ruedin, Weaver, Roth \& Widmer, 2009) arrive aux mêmes résultats (30\% des infirmières sont âgées de 50 ans et plus). C'est donc un tiers du personnel infirmier qu'il faudra remplacer au cours des 15 prochaines années, auquel les auteurs du rapport ajoutent encore une hausse de 12\% des équivalents temps plein liée à l'évolution démographique.

${ }^{14} \mathrm{EMS}$ : institutions pour personnes âgées (équivalent aux $\mathrm{EHPAD}$ en France).

${ }^{15}$ Parmi les infirmières de notre échantillon, $17 \%$ ont un diplôme de base de type bachelor.
} 
(19\%) ont suivi des formations dont la durée n'excède pas quelques jours, la majorité $(56 \%)$ a suivi une formation de type Certificate of Advanced Studies (CAS). Quelques infirmières $(5 \%)$ ont suivi une formation de niveau bachelor après leur formation de base, et une petite minorité a poursuivi jusqu'au niveau master $(1,6 \%)$ et doctorat (3 individus).

\section{Une force de travail étrangère}

Les pays occidentaux connaissent une pénurie de personnel infirmier depuis plusieurs décennies. Les stratégies de recrutement que développent les pays européens pour faire face à cette pénurie sont fortement dépendantes de leur positionnement dans l'ordre politique régional et global. Dans une étude comparative, Angèle Mendy (2018) montre que le Royaume-Uni a longtemps recruté son personnel soignant au sein des pays du Commonwealth (et notamment l'Afrique du Sud), alors que la France a privilégié le recrutement d'infirmières dans les départements d'outre-mer (Réunion, Martinique et Guadeloupe). Quant à la Suisse, le recrutement d'infirmières étrangères a véritablement débuté au début des années 1990, puis a connu une nette accélération en 2002, suite à l'adoption de la loi sur la libre circulation des personnes. Cette augmentation du nombre d'infirmières étrangères est symptomatique des modifications structurelles ayant affecté la migration en Suisse, qui a vu une main-d'œuvre étrangère très qualifiée issue de pays limitrophes comme l'Allemagne et la France s'ajouter - et dans certains cas même remplacer - à la migration traditionnelle faiblement qualifiée issue des pays du Sud de l'Europe (Italie, Espagne, Portugal, pays de l'Ex-Yougoslavie) (Оesch, 2013).

Bien que la thématique des infirmières étrangères apparaisse régulièrement dans les médias, la distribution de cette main d'œuvre au sein de la profession infirmière demeure largement méconnue. Notre recherche permet d'en éclairer certains aspects. 
Plus du tiers (38,3\%) des infirmières qui exercent en Suisse romande sont de nationalité étrangère ${ }^{16}$, soit une proportion supérieure à celle des étrangers parmi la population active en Suisse durant la période de l'enquête $(30,1 \%$ en 2014 selon l'OFS). Les nationalités sont très diverses: pas moins de 38 pays sont représentés, allant des États-Unis à la Roumanie, en passant par le Maroc, la Syrie, l'Érythrée ou encore le Brésil. Mais cette diversité des nationalités doit cependant être relativisée, la grande majorité $(82,4 \%)$ des infirmières étrangères étant de nationalité française.

La distribution des infirmières étrangères n'est de loin pas homogène (voir tableau A3 en annexe 3). Sans surprise, les cantons ayant une frontière commune avec la France (Genève, Neuchâtel et Jura) se caractérisent par des taux d'infirmières étrangères plus élevés que les autres. Par ailleurs, alors que certains secteurs hospitaliers (anesthésie, bloc opératoire, soins intensifs) présentent une forte surreprésentation d'infirmières étrangères, certains secteurs extrahospitaliers (santé scolaire, enseignement et recherche, soins à domicile) apparaissent au contraire comme de véritables bastions privilégiant le recrutement d'infirmières de nationalité suisse.

On constate également que le taux d'infirmières étrangères est nettement plus élevé parmi les hommes (48,8\%) que parmi les femmes $(36,3 \%)$, et qu'il connaît un pic parmi celles qui sont âgées de 30 à 39 ans (46,4\%), pour redescendre régulièrement parmi les catégories d'âge supérieures. Cette surreprésentation de profils jeunes et masculins correspond à ceux que l'on retrouve dans les secteurs les plus médicalisés.

Parallèlement à l'augmentation du nombre de travailleurs de nationalité étrangère, la Suisse a connu une hausse du nombre de travailleurs frontaliers, leur nombre étant passé de

\footnotetext{
${ }^{16}$ Si l'on ajoute les infirmières de nationalité française qui détiennent aussi la nationalité suisse, cette proportion s'établit à $41,3 \%$.
} 
Tableau 1. Taux d'infirmières frontalières selon les cantons (\%)

\begin{tabular}{|l|c|}
\hline Genève & 64,9 \\
\hline Jura & 33,7 \\
\hline Neuchâtel & 27,8 \\
\hline Vaud & 14,0 \\
\hline Berne & 9,9 \\
\hline Valais & 9,0 \\
\hline Fribourg & 0,0 \\
\hline
\end{tabular}

198000 durant la période 2005-2007 à 308000 pour la période 2014-2016 (OFS, 2019). Parmi les infirmières qui exercent en Suisse romande, $27 \%$ résident en France. Ce taux est bien sûr particulièrement élevé dans les cantons frontaliers, et tout particulièrement à Genève où il s'élève à près de $65 \%$ (tableau 1).

Toutes les infirmières frontalières ne sont pas de nationalité étrangère: on y trouve en effet $14,5 \%$ d'infirmières suisses, proportion qui grimpe même à $18,3 \%$ dans le canton de Genève.

\section{Le revenu}

En comparaison européenne, la Suisse se distingue par des conditions de travail avantageuses, notamment au niveau des salaires. Selon nos données, le revenu moyen des infirmières représente $125 \%$ du revenu moyen de la population active, soit un écart plus important que celui observé parmi les pays de l'OCDE $(114 \%)^{17}$. Bien que les infirmières travaillant en Suisse

${ }^{17}$ Précisons qu'aucune donnée relative au revenu des infirmières suisses ne figure dans les rapports de l'OCDE. 
Tableau 2. Revenu net, revenu équivalent plein temps, taux d'activité

\begin{tabular}{|l|c|c|c|c|}
\cline { 2 - 5 } \multicolumn{1}{c|}{} & N & Moyenne & Écart-type & Médiane \\
\hline Revenu (en CHF) & 2421 & 5190 & 1694 & 5000 \\
\hline $\begin{array}{l}\text { Revenu équivalent } \\
\text { plein temps (en CHF) }\end{array}$ & 2420 & 6434 & 1423 & 6250 \\
\hline Taux d'activité (en \%) & 2914 & 80,4 & 19,2 & 80 \\
\hline
\end{tabular}

romande bénéficient d'une formation de base similaire, nous observons des disparités de revenu relativement importantes au sein de la profession infirmière. Le taux d'activité apparaît comme l'une des principales explications à ces disparités. D'autres facteurs tels que l'ancienneté, la formation suivie après le diplôme de base, le statut professionnel, le secteur ou le type d'établissement, et même le canton de travail sont également susceptibles de peser sur le niveau de revenu.

En 2014, le revenu mensuel net moyen des infirmières en Suisse romande s'élève à $5190 \mathrm{CHF}$, le revenu médian à $5000 \mathrm{CHF}$ et le taux d'activité moyen à $80,4 \%$ (tableau 2). Le revenu équivalent plein temps moyen monte à $6434 \mathrm{CHF}^{18}$.

Le tableau A4 (voir annexe 3) présente les différences de revenu selon de nombreuses caractéristiques. Un statut de cadre (+1 $248 \mathrm{CHF})$ et le fait d'avoir suivi une formation après le diplôme de base (+860 CHF) augmentent substantiellement la rémunération du travail. Être de nationalité suisse $(+481 \mathrm{CHF})$, résider en Suisse (+312 CHF), avoir obtenu son diplôme en

\footnotetext{
${ }^{18}$ Le revenu équivalent plein temps correspond à l'hypothèse que toutes les infirmières travaillent à $100 \%$ en 2014 . Il permet de comparer la rémunération mensuelle du travail salarié et d'évaluer les inégalités salariales au sein de la profession infirmière. Il ne prend pas en compte le taux d'activité qui affecte le revenu net.
} 
Suisse $(+215 \mathrm{CHF})$ et être un homme $(+169 \mathrm{CHF})$ renforcent également le revenu, mais dans une moindre mesure.

Les disparités cantonales en matière de revenu équivalent plein temps moyen découlent vraisemblablement à la fois du niveau de vie du canton et de la surreprésentation d'infirmières hautement qualifiées dans certains cantons. La palme de la rémunération des infirmières revient à Fribourg et Genève avec des revenus moyens équivalent plein temps de respectivement $7012 \mathrm{CHF}$ et $6682 \mathrm{CHF}$. Tous les autres cantons affichent un revenu moyen variant entre 6140 et $6415 \mathrm{CHF}$.

Des disparités de revenu s'observent selon le type d'établissement. Dans les hôpitaux universitaires cantonaux, le revenu moyen s'élève à $6427 \mathrm{CHF}$, alors qu'il est de $6106 \mathrm{CHF}$ dans les hôpitaux de zone ou régionaux, et de $5791 \mathrm{CHF}$ dans le privé (clinique privée, etc.). Enfin, la rémunération du travail augmente significativement avec l'ancienneté de l'infirmière (mesurée par le nombre d'années depuis l'obtention du diplôme) à raison de $73 \mathrm{CHF}$ par année d'ancienneté.

Le graphique 1 présente les revenus équivalents plein temps par secteur d'exercice. Les secteurs affichant les salaires mensuels les plus élevés sont, dans l'ordre, la direction (8 $611 \mathrm{CHF}$ ), ES/HES (8 $258 \mathrm{CHF}$ ) et Enseignement sur le terrain (8 $082 \mathrm{CHF}$ ). À l'inverse, les secteurs les moins rémunérateurs sont, dans l'ordre, la salle de réveil (5 $766 \mathrm{CHF})$, la gynécologie (5 $900 \mathrm{CHF}$ ) et la médecine générale (5944 CHF).

Certains secteurs considérés comme prestigieux, tels que les urgences ou la chirurgie (voir chapitre 4), sont moins rémunérateurs comparés à d'autres secteurs considérés comme beaucoup moins prestigieux. Cela s'explique en partie par des effets de composition. Certains secteurs relativement médicalisés (et donc prestigieux) comportent davantage d'infirmières ayant peu d'expérience, car ils constituent en quelque sorte un passage "obligé» ou du moins typique du début de carrière (voir chapitre 9). Dans ce cas, une part des 
Graphique 1. Revenu équivalent plein temps selon le secteur

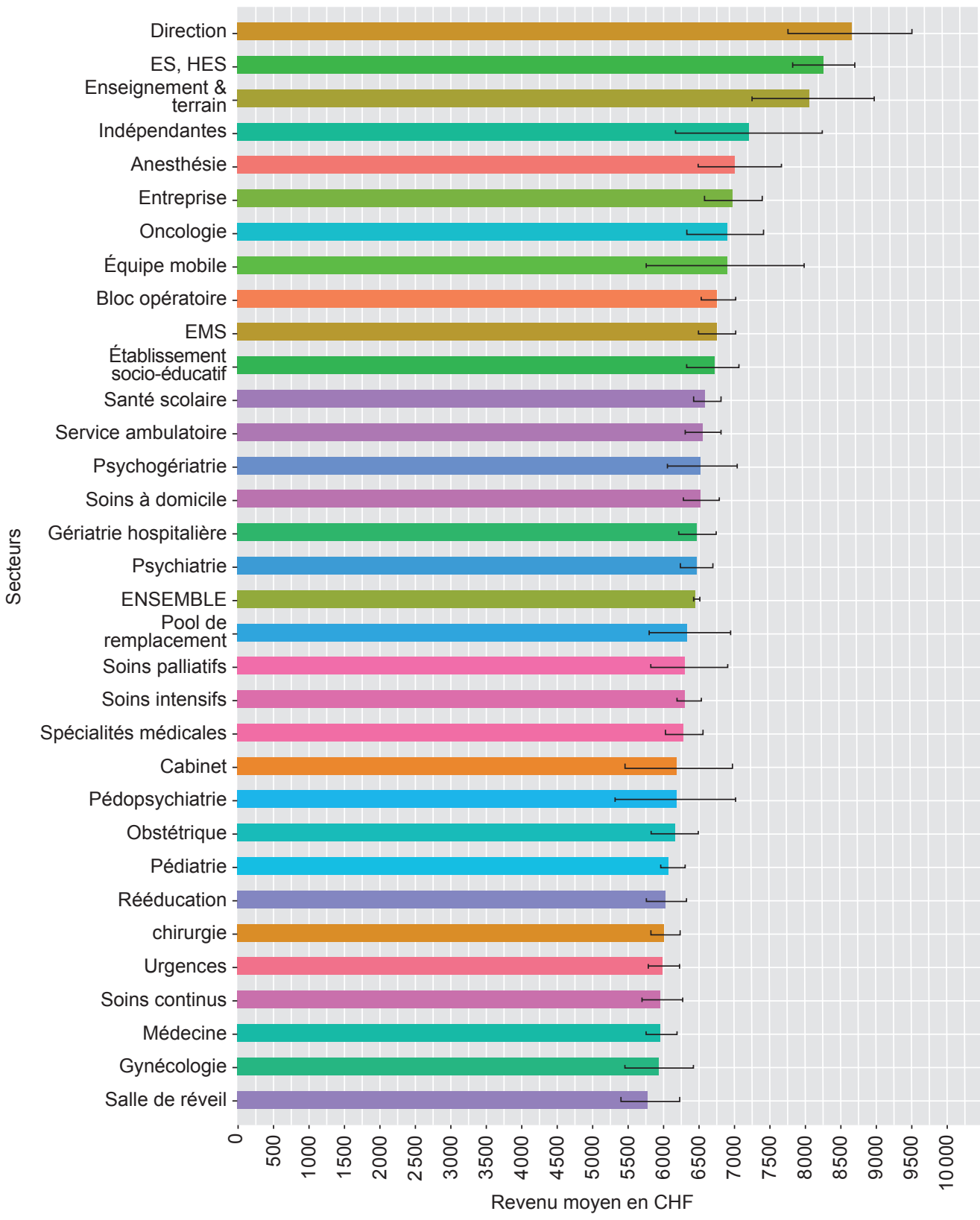

Note: Données agrégées par secteur; intervalle de confiance à 95\%. 
différences de salaire entre certains secteurs s'explique par des différences de profil des infirmières: les secteurs composés de beaucoup de jeunes infirmières rémunèrent moins celles-ci du fait de leur manque d'expérience professionnelle (l'ancienneté), alors que les secteurs composés d'infirmières disposant d'une grande expérience professionnelle les rémunèrent mieux. Ainsi, pour les secteurs employant de jeunes infirmières, la moyenne du revenu équivalent plein temps sera affectée par le déficit d'expérience professionnelle et le principe de rémunération de l'ancienneté - une faible expérience professionnelle justifiant, selon ce principe, une rémunération mensuelle inférieure.

En contrôlant les effets de composition tels que l'ancienneté moyenne différentielle entre les secteurs, une analyse de régression ${ }^{19}$ permet d'évaluer si un secteur est plus rémunérateur qu'un autre. En introduisant plusieurs variables explicatives, elle permet par exemple d'évaluer si le revenu moyen des urgences est inférieur à celui de l'anesthésie lorsque l'on compare des infirmières ayant le même profil (une expérience, un diplôme, une fonction, etc. identiques).

Dans notre modèle d'explication du revenu équivalent plein temps, toutes les variables explicatives sont intégrées simultanément (voir tableau A5 en annexe 3). Sous contrôle des autres variables, les cadres affichent un différentiel de revenu important (+867 CHF) avec les infirmières non-cadres. Un diplôme obtenu à l'étranger fait diminuer le revenu $(-226 \mathrm{CHF})$, tout

\footnotetext{
${ }^{19}$ L'analyse de régression renvoie à un ensemble de techniques statistiques de modélisation. La modélisation statistique cherche à expliquer ou à prédire une variable à partir d'une ou de plusieurs variables. Plus précisément, ces techniques statistiques permettent d'estimer les effets spécifiques (parfois appelés effets purs ou nets) de chaque variable explicative introduite dans le modèle, en neutralisant l'effet des autres variables explicatives sur la variable à expliquer. Le choix d'utiliser un modèle de régression linéaire ou un modèle de régression logistique multinomiale dépend du niveau de mesure de la variable à expliquer (quantitatif pour le premier et nominal pour le second).
} 
comme le fait de résider en France (-177 CHF). L'ancienneté agit positivement sur le revenu (66 CHF supplémentaires par année d'ancienneté), ainsi que le fait d'avoir suivi une formation après le diplôme de base (+209 CHF) et le fait d'être un homme $(+311 \mathrm{CHF})$, ce qui dénote l'existence d'une inégalité salariale entre femmes et hommes au sein de la profession infirmière. Le canton de travail exerce un effet sur le revenu: il est préférable de travailler à Genève (366 CHF de plus que dans le canton de Vaud, la catégorie de référence).

\section{L'origine sociale}

Un dernier enseignement des données recueillies concerne le recrutement social de la profession. Alors que certaines professions telles que celles de journaliste (LAFARGe \& MARCHETti, 2011) ou de pompier (Pudal, 2016) recrutent dans des régions spécifiques de l'espace social, la profession infirmière a, semblet-il, toujours recruté de façon relativement éparse, en France (Picot, 2005) comme au Canada par exemple (McPherson, 2003).

Si l'on compare nos résultats concernant les infirmières de Suisse romande avec ceux du volet suisse de l'enquête sociale européenne (ESS7) de 2014² (tableau 3), on constate que les infirmières ont plus souvent des parents ayant effectué des études longues que la population résidente en Suisse, qu'il s'agisse des études secondaires et universitaires (respectivement 14,8 vs 10,1\%) ou des formations professionnelles supérieures $(25,8 \%$ vs $16,9 \%)$. De manière cohérente, les infirmières sont nettement moins nombreuses à avoir des parents au bénéfice d'un CFC (36,8\%) que la population résidente en Suisse (46,7\%).

\footnotetext{
${ }^{20}$ Disponible sur http://www.europeansocialsurvey.org.
} 
Tableau 3. Niveau de formation des parents $(\%)^{21}$

\begin{tabular}{|l|r|r|c|c|}
\cline { 2 - 5 } \multicolumn{1}{c|}{} & EPRIS $^{\mathbf{a}}$ & ESS7 & Différence & p \\
\hline Obligatoire & 16,5 & 23,9 & $-7,4$ & $<0,001$ \\
\hline CFC & 36,8 & 46,7 & $-9,9$ & $<0,001$ \\
\hline Maturité & 6,1 & 2,4 & 3,7 & $<0,001$ \\
\hline $\begin{array}{l}\text { Formation professionnelle } \\
\text { supérieure }\end{array}$ & 25,8 & 16,9 & 8,9 & $<0,001$ \\
\hline Université & 14,8 & 10,1 & 4,7 & $<0,001$ \\
\hline
\end{tabular}

Source: EPRIS et ESS7.

a Enquête sur les pratiques et les représentations des infirmières en Suisse romande. ${ }^{b} \mathrm{HES}, \mathrm{ES}, \mathrm{ETS}$, brevet, maîtrise.

Tableau 4. Catégorie socioprofessionnelle des parents (\%) $)^{22}$

\begin{tabular}{|l|r|r|c|c|}
\cline { 2 - 5 } \multicolumn{1}{c|}{} & EPRIS & ESS7 & Différence & p \\
\hline $\begin{array}{l}\text { Professions libérales } \\
\text { et grands employeurs }\end{array}$ & 7,1 & 5,3 & 1,7 & 0,031 \\
\hline Petits indépendants & 20,0 & 26,1 & $-6,1$ & $<0,001$ \\
\hline Professions techniques & 12,2 & 8,5 & 3,7 & $<0,001$ \\
\hline Ouvriers & 19,0 & 28,2 & $-9,2$ & $<0,001$ \\
\hline Managers & 20,4 & 13,7 & 6,7 & $<0,001$ \\
\hline Employés & 5,2 & 5,7 & $-0,4$ & 0,557 \\
\hline Professions socioculturelles & 8,9 & 6,7 & 2,2 & 0,014 \\
\hline Travailleurs des services & 7,2 & 5,9 & 1,3 & 0,102 \\
\hline
\end{tabular}

Source: EPRIS et ESS7.

a Nomenclature selon le schéma de classe de Daniel Oesch (2006).

${ }^{21}$ Selon le plus haut niveau de formation du père ou de la mère.

${ }^{22}$ Selon la plus haute catégorie socioprofessionnelle du père ou de la mère. 
Le même constat est valable concernant la classe d'origine (tableau 4). Alors que 19\% des infirmières ont des parents ouvriers, ce taux s'élève à $28,2 \%$ pour la population suisse. À l'opposé de l'échelle sociale, la proportion d'infirmières ayant au moins un des deux parents occupant une position de manager s'élève à 20,4\%, contre $13,7 \%$ dans l'ensemble de la population. La profession infirmière a donc tendance à recruter dans des régions de l'espace social un peu plus élevées que la moyenne suisse. 


\section{Chapitre 3}

\section{L'espace infirmier $^{23}$}

La théorie des champs de Pierre Bourdieu nous a permis d'établir une rupture avec l'image d'une profession homogène dans laquelle tous les individus seraient tendus vers un même objectif: celui d'une émancipation de la profession ${ }^{24}$. Nos analyses permettent en effet de prendre la mesure des enjeux de luttes qui distinguent les individus. Se dessine ainsi un espace professionnel différencié dans lequel les individus occupent des positions tendanciellement associées à des prises de position concourantes ou divergentes, notamment en ce qui concerne la définition des pratiques légitimes du métier.

Les champs peuvent se définir "comme des espaces structurés de positions (ou de postes) dont les propriétés dépendent de leur position dans ces espaces et qui peuvent être analysées indépendamment des caractéristiques de leurs occupants (en partie déterminées par

${ }^{23}$ Ce chapitre est en partie tiré de Longchamp, Toffel, Bühlmann \& Tawfik (2018) et Toffel (2018).

${ }^{24} \mathrm{Si}$ la mobilisation de la théorie de champs ne va pas de soi pour étudier une profession, de nombreuses études ont pourtant démontré les gains heuristiques de ce cadre analytique appliqué à des groupes professionnels. Voir Gaspar (2012), Mathieu (2000), Pichonnaz \& Toffel (2018), Pinell (2009), Pinto (2007) et Toffel (2018). 
elles)»(Bourdieu, 1984b, p. 113). Les individus qui participent à un champ luttent pour la possession ou la transformation des différentes formes de «capital» (ou ressources) qui le caractérisent. En insistant sur les rapports conflictuels qui opposent les occupants des différentes positions, la théorie des champs permet ainsi de penser ensemble l'unité et la diversité et d'analyser les "relations structurales» qui, tout en intégrant les interactions de face-à-face, lient aussi entre eux des individus qui "peuvent ne jamais se rencontrer, voire s'ignorer méthodiquement, et rester profondément déterminés, dans leur pratique, par la relation d'opposition qui les unit» (Bourdieu, 1992, p. 303-304).

Un champ est un espace de luttes relativement spécifiques prenant place autour de ressources inégalement distribuées. Déterminé par la position qu'il occupe par rapport aux autres champs, son degré d'autonomie se mesure "à l'importance de l'effet de retraduction ou de réfraction que sa logique spécifique impose aux influences ou aux commandes externes" (BourdiEu, 1992, p. 306). Comme nous le verrons, les deux formes de capital que l'on peut identifier au sein de la profession infirmière ne suffisent pas à lui conférer l'autonomie suffisante permettant de parler d'un "champ infirmier" à part entière. Mais il n'en demeure pas moins que cette profession réunit au moins deux propriétés d'un champ. Premièrement, l'une de ses caractéristiques est précisément la lutte pour une autonomie à l'égard du champ médical par l'identification et la formation d'enjeux propres. Deuxièmement, si la profession infirmière apparaît comme très hétérogène en raison de ses nombreux secteurs d'activité, toutes les infirmières se trouvent néanmoins liées entre elles, non seulement par une définition institutionnellement sanctionnée par un diplôme reconnu par l'État, mais aussi par l'existence d'enjeux communs qui concernent directement la définition légitime de la pratique des soins. La profession infirmière est donc traversée par des luttes qui, sans être nécessairement le produit d'une conscience, présupposent néanmoins que les antagonistes soient d'accord 
sur leurs points de désaccord. L'addition de ces deux propriétés nous autorise à considérer cette profession comme un espace social présentant certaines propriétés du champ.

Il est ainsi possible de parler d'un espace infirmier, mais sans le qualifier de "champ" en raison d'une autonomie trop faible. Il s'avère dès lors nécessaire de saisir les principales forces dans lesquelles s'insère cet espace, et en particulier celles du champ médical.

\section{L'espace infirmier et le champ médical: un rapport de domination}

C'est sous l'angle de l'asymétrie des rapports qu'elle entretient avec la profession médicale que la profession infirmière a généralement été étudiée. Qu'ils soient historiques ou sociologiques, les travaux ont le plus souvent décrit un tandem médecin-infirmière dans lequel cette dernière est définie comme "un pur instrument au service du médecin» (Poisson, 2008, p. 56). Dans son ouvrage désormais classique, Everett Hughes (1996) soutient que la position dominée des infirmières repose essentiellement sur un modèle pratique de division du travail: "D'une certaine manière, le travail d'infirmière comprend tout ce qui doit être fait dans un hôpital, mais qui n'est pas fait par d'autres catégories de personnes» (p. 70). Attaché à saisir le pendant hiérarchique de cette division du travail, Eliot Freidson (2007) montre quant à lui que la domination des médecins repose sur la prérogative dont ces derniers détiennent le monopole légal et symbolique; celle de l'évaluation des maux dont souffrent les patients et de la décision des traitements à appliquer. Geneviève Picot (2005) rejoint ces observations: bien que la féminisation de la profession médicale et la (relative) masculinisation de la profession infirmière entraînent "de nouvelles modalités en termes de rapport de genre» (p. 98), les rapports sociaux entre médecins et infirmières restent 
marqués par la domination des premiers sur les secondes. Pour Isabelle Feroni et Anémone Kober (1995), la profession infirmière ne jouit que d'une "autonomie limitée» (p. 37) en raison du monopole médical sur le diagnostic, la prescription et l'incision des corps. De ce point de vue, les médecins ont l'ascendant sur l'ensemble des professions paramédicales, et notamment sur les infirmières puisque celles-ci ne peuvent avoir un accès direct - ou alors restreint à des "actes simples" - à leurs patients sans l'intermédiaire du médecin. Par conséquent, en suivant les travaux de Feroni et Kober, on peut dire que si les infirmières partagent avec les professions établies le monopole de leur titre, elles s'en distinguent cependant par une autonomie très limitée en raison des régulations externes dont elles sont l'objet, à la fois par la profession médicale et par l'État.

La description du champ médical représente donc un préalable nécessaire à celle de l'espace infirmier. Patrice Pinell (2009) identifie trois sous-espaces du champ médical: "l'espace de la médecine clinique", "l'espace de la médecine sociale" et "l'espace des sciences accessoires». Étant soumis à des demandes sociales différentes et entretenant des relations distinctes avec l'État, ces trois sous-espaces sont régis par des finalités particulières. De ce point de vue, l'«espace de la médecine clinique» apparaît non seulement comme le plus autonome, mais aussi comme le plus dominant au sein du champ médical. Il constitue en effet "l'espace de production le plus autonome de "biens de santé" à la fois parce que la caractérisation qui y est faite des maladies est proprement médicale (elle n'emprunte à aucun autre domaine de savoir) et parce que l'État, reconnaissant cette caractérisation comme la seule légitime, a octroyé aux médecins le monopole des pratiques médicales légitimes»(p. 342-343). C’est donc principalement ce sous-espace qui fait autorité et qui, comme nous le verrons, détermine une partie considérable des pratiques légitimes pour la profession infirmière.

Ainsi, et bien que les infirmières administrent des soins aux malades sur la base de savoirs et savoir-faire professionnels, les 
décisions afférentes à la prise en charge des patients au niveau de leurs affections - diagnostics et traitements - demeurent sous stricte prescription médicale. Aussi, et quand bien même la profession dispose d'une formation spécifique, de la reconnaissance d'un diplôme décerné par l'État, d'un rôle déterminé, et même d'une hiérarchie propre, les rapports professionnels qu'entretiennent les infirmières au sein de leur profession et avec les professions voisines restent marqués par la domination médicale; légalement mais aussi symboliquement. En fait, on peut poser qu'en termes de structuration, l'espace infirmier est largement déterminé par un champ médical qui continue de représenter un point de référence dans les possibilités d'envisager les pratiques les plus légitimes en matière de soins. L'espace infirmier reproduit en effet la plupart des oppositions structurant le champ médical: hiérarchie des secteurs d'activité et rapports aux savoirs de type biomédical notamment (voir le chapitre 4).

Considérer que l'espace infirmier se trouve tout entier inscrit dans le champ médical permet donc d'éclairer la nature des relations entre infirmières et médecins. Bien sûr, les champs étant entre autres caractérisés par des luttes permanentes - donc $\mathrm{du}$ changement - ces rapports ne sont pas immuables. À ce titre, on peut relever que le paysage politique et économique a, depuis les recherches citées plus haut, subi des transformations substantielles dont les effets sur les professions de santé sont importants. Bien que les relations entre médecins et infirmières restent marquées par un rapport social dans lequel les secondes se trouvent dominées tant légalement que symboliquement par les premiers, les transformations survenues depuis la fin des années 1980 dans l'espace de la santé - raccourcissement des séjours hospitaliers, "virage ambulatoire", etc.; toutes choses qui participent d'un processus de néolibéralisation de cet espace (Pierru, 2008) - ont des effets tant sur le champ médical que sur l'espace infirmier. 


\section{Deux formes de capital efficientes au sein de l'espace infirmier}

Un champ est un espace structuré selon un certain nombre de ressources, ou capitaux, qui, parce que relativement rares, font l'objet d'enjeux spécifiques. Deux catégories de capital peuvent être distinguées: les espèces fondamentales (ressources économiques, culturelles et sociales) d'une part, qui sont efficientes dans tous les champs; les espèces spécifiques d'autre part, qui n'exercent leur efficacité que dans le périmètre du champ par et pour lequel elles existent.

L'enquête par entretiens nous a permis d'identifier deux formes de capital particulièrement efficientes au sein de l'espace infirmier: le "capital médical» et le "capital infirmier». Nous définissons le capital médical comme l'ensemble des ressources acquises par la pratique autant que par la formation, et relatives à la physiopathologie, à la pharmacologie et à la maîtrise de gestes techniques impliquant le plus souvent la manipulation d'instruments ou de machines. Le capital infirmier désigne quant à lui l'ensemble des ressources issues des sciences humaines et sociales, des sciences infirmières ou des savoirs paramédicaux, acquises par la pratique autant que par la formation et dont la mise en œuvre permet non seulement une prise en charge des patients, mais aussi une meilleure autodétermination professionnelle ${ }^{25}$. Les deux formes de capital que nous identifions ne sont pas de même statut. Parce qu'issu du champ médical, le capital médical dispose certes de la légitimité que lui confèrent ses origines, mais n'est toutefois pas spécifique à l'espace infirmier. Quant au capital infirmier, il est à considérer comme un capital en voie de constitution, son accession au statut de capital spécifique étant conditionnée à l'existence d'un véritable

\footnotetext{
${ }^{25}$ Le lecteur trouvera en annexe 2 un exposé de la démarche suivie pour mesurer les capitaux médical et infirmier dans le questionnaire.
} 
"champ infirmier» qui, comme nous le montrerons, n'est pas encore d'actualité26.

En proposant deux formes originales de capital, nous n'entendons nullement contribuer à l'inflation conceptuelle qui entoure cette notion. Suivant en cela Erik Neveu (2013), nous considérons que les formes spécifiques de capital, bien qu'utiles pour caractériser les enjeux de certains espaces sociaux, ne sont cependant pas autre chose que des formes dérivées ou combinatoires des trois espèces fondamentales. Les capitaux médical et infirmier peuvent ainsi être considérés comme des formes spécifiques de capital culturel qui trouvent dans l'espace infirmier leur terrain d'efficience et contribuent à sa structuration.

\section{L'espace infirmier $^{27}$}

Une analyse de correspondances multiples (ACM) a permis de construire l'espace professionnel au sein duquel exercent les infirmières (graphique 2). Basée sur une logique de décomposition géométrique, l'ACM est une méthode statistique qui produit une image simplifiée des multiples relations entre les capitaux (ici les capitaux médial et infirmier) des individus (Le Roux \& Rounnet, 2010). En projetant les variables (qui permettent de mesurer les capitaux) ainsi que les individus (ici les infirmières qui ont répondu au questionnaire) sur un plan géométrique, cette méthode permet de visualiser

\footnotetext{
${ }^{26}$ Le capital infirmier étant en voie de constitution, la rigueur exigerait le recours systématique aux guillemets lorsqu'il est mentionné. Nous y avons cependant renoncé pour d'évidentes raisons de lisibilité. Pour un développement de la distinction entre champ et espace et ce qu'elle implique pour le concept de capital, voir Toffel (2018).

${ }^{27}$ Une première esquisse de l'espace infirmier a été proposée sur la base de l'analyse des entretiens (Longchamp \& Toffel, 2016). Ces résultats ont été confirmés et précisés dans Longchamp, Toffel, BüHLmann \& Tawfik (2018) et Toffel (2018).
} 
Graphique 2. L'espace infirmier (version simplifiée)

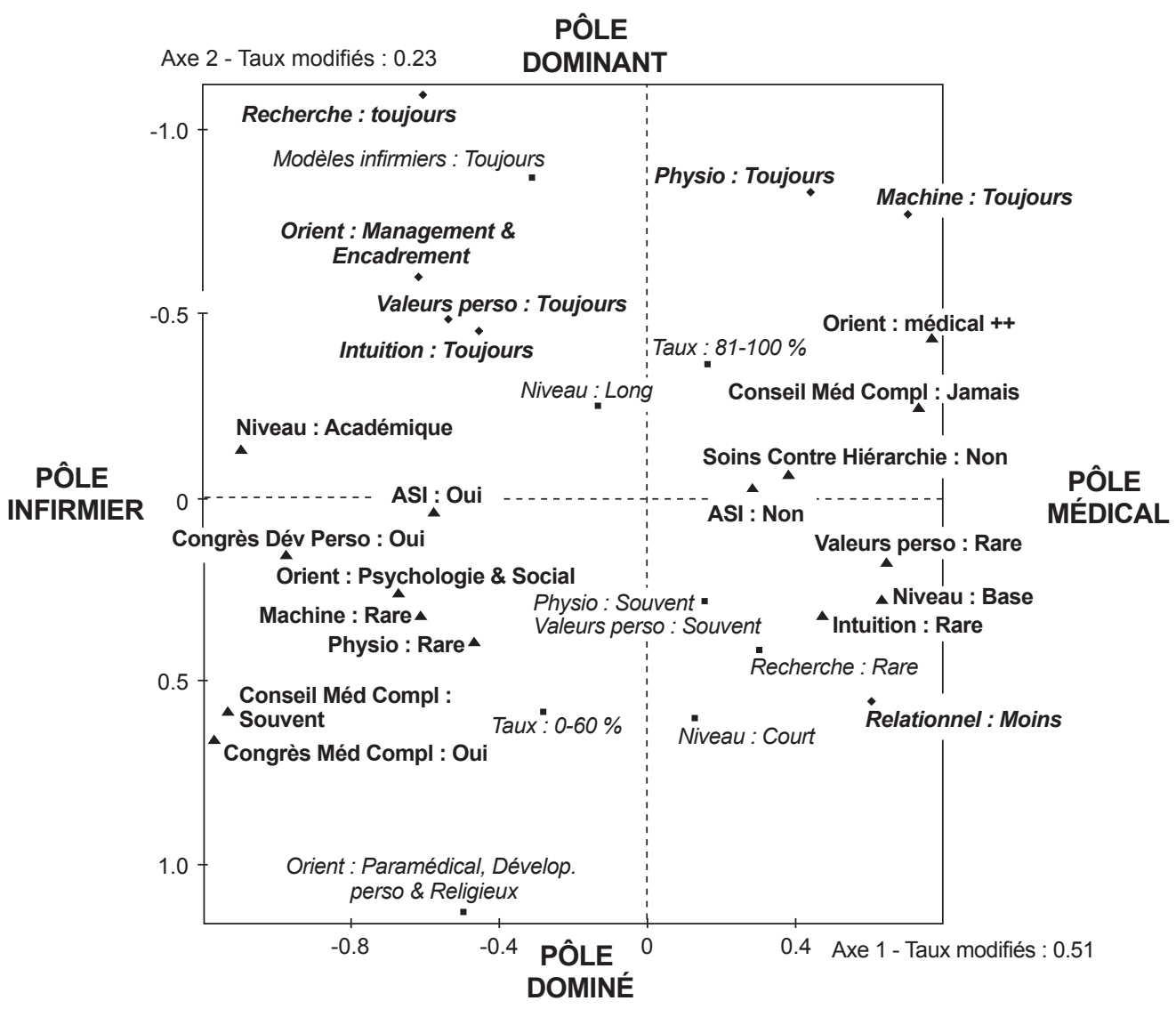

Source: Enquête 2014 sur les pratiques et les représentations des infirmières en Suisse romande (EPRIS).

Note: Notre échantillon est composé de 2923 individus. Seules figurent sur ce graphique les modalités retenues pour l'interprétation des axes, soit celles dont la contribution est égale ou supérieure à la contribution moyenne (pour la version complète, voir graphique A1 en annexe 4). Les modalités en gras (triangles) sont celles retenues pour l'interprétation du premier axe (horizontal). Les modalités en italique (carrés) sont celles retenues pour l'interprétation du deuxième axe (vertical). Les modalités en gras italique (losanges) sont celles retenues pour l'interprétation des deux axes. 
l'ensemble de la structure des positions de l'espace infirmier et d'en dégager les principales oppositions. L'ACM permet ainsi de révéler des contiguïtés, des proximités, des oppositions et des distances entre les infirmières caractérisées par leurs capitaux.

Seize indicateurs des capitaux médical et infirmier forment les variables actives de notre ACM qui totalisent 46 modalités de réponses ${ }^{28}$.

Le premier axe (horizontal) et le deuxième axe (vertical) de l'ACM renvoient respectivement à la structure et au volume des deux formes de capital efficientes au sein de l'espace infirmier.

Les infirmières situées à gauche du premier axe (graphique 2) présentent une structure de capital à dominante infirmière. Elles mobilisent toujours leurs connaissances en recherche, leurs valeurs personnelles et leur intuition, mais rarement leurs connaissances en physiopathologie et en utilisation de machines. Elles ont suivi une formation postgrade de niveau académique, à orientation management et encadrement ou psychologie et social. Membres de l'Association suisse des infirmiers et infirmières (ASI), leur inclination pour des pratiques hétérodoxes se manifeste notamment par le fait qu'elles assistent à des congrès de développement personnel et de médecine complémentaire, ou encore par le fait qu'elles n'hésitent pas à orienter leurs patients vers des spécialistes de médecine complémentaire.

À l'opposé, les infirmières situées à droite du premier axe présentent une structure inverse de capital, à dominante médicale. Elles mobilisent toujours leurs connaissances en physiopathologie et en utilisation de machines, mais rarement leurs valeurs personnelles, leur intuition ou leurs compétences relationnelles. Beaucoup n'ont pas suivi de formation après leur diplôme de base, mais celles qui en ont suivi ont choisi une orientation fortement

${ }^{28}$ Le tableau A11 en annexe 4 présente les contributions de ces variables et modalités aux deux premiers axes de l'ACM. 
médicale. Non membres de l'ASI, elles inscrivent leur pratique dans une certaine orthodoxie médicale, comme en atteste le fait qu'elles n’orientent jamais leurs patients vers des spécialistes de médecine complémentaire et qu'elles ne livrent pas de soins allant à l'encontre des consignes de leur employeur ou de leur hiérarchie. En opposant les infirmières bien dotées en "capital infirmier" mais faiblement dotées (relativement) en capital médical, aux infirmières présentant des caractéristiques inverses, ce premier axe révèle une structure en chiasme de l'espace infirmier qui nous autorise à parler d'un "pôle infirmier» (à gauche) et d'un "pôle médical» (à droite).

Le deuxième axe oppose les infirmières qui présentent un fort volume des deux formes de capital à celles qui en présentent un faible. On trouve en haut de l'espace les infirmières qui mobilisent toujours leurs valeurs personnelles, leur intuition, ou encore leurs connaissances en recherche, en modèles infirmiers, en physiopathologie et en utilisation de machines. Elles ont suivi des formations de niveau long et leur taux d'activité élevé agit comme une ressource favorisant l'accumulation de capital. À l'opposé, les infirmières situées en bas de l'espace, qui ont suivi une formation courte - souvent de type paramédical, développement personnel ou religieux - et ne présentent qu'un faible taux d'activité, ne mobilisent que rarement leurs compétences relationnelles ou leurs connaissances en recherche, et souvent leurs valeurs personnelles et leurs connaissances en physiopathologie. À la différence de ce que l'on observe pour le premier axe, le seul critère d'opposition est ici l'intensité de la mobilisation de capital, indépendamment du type de capital mobilisé. On peut par conséquent parler d'un "pôle dominant" (en haut) et d'un "pôle dominé» (en bas).

\section{Un espace de positions}

Les différentes positions de l'espace infirmier peuvent être matérialisées en ajoutant les 32 secteurs d'activité dans lesquels exercent les infirmières de notre échantillon (graphique 3). 
Graphique 3. L'espace des positions

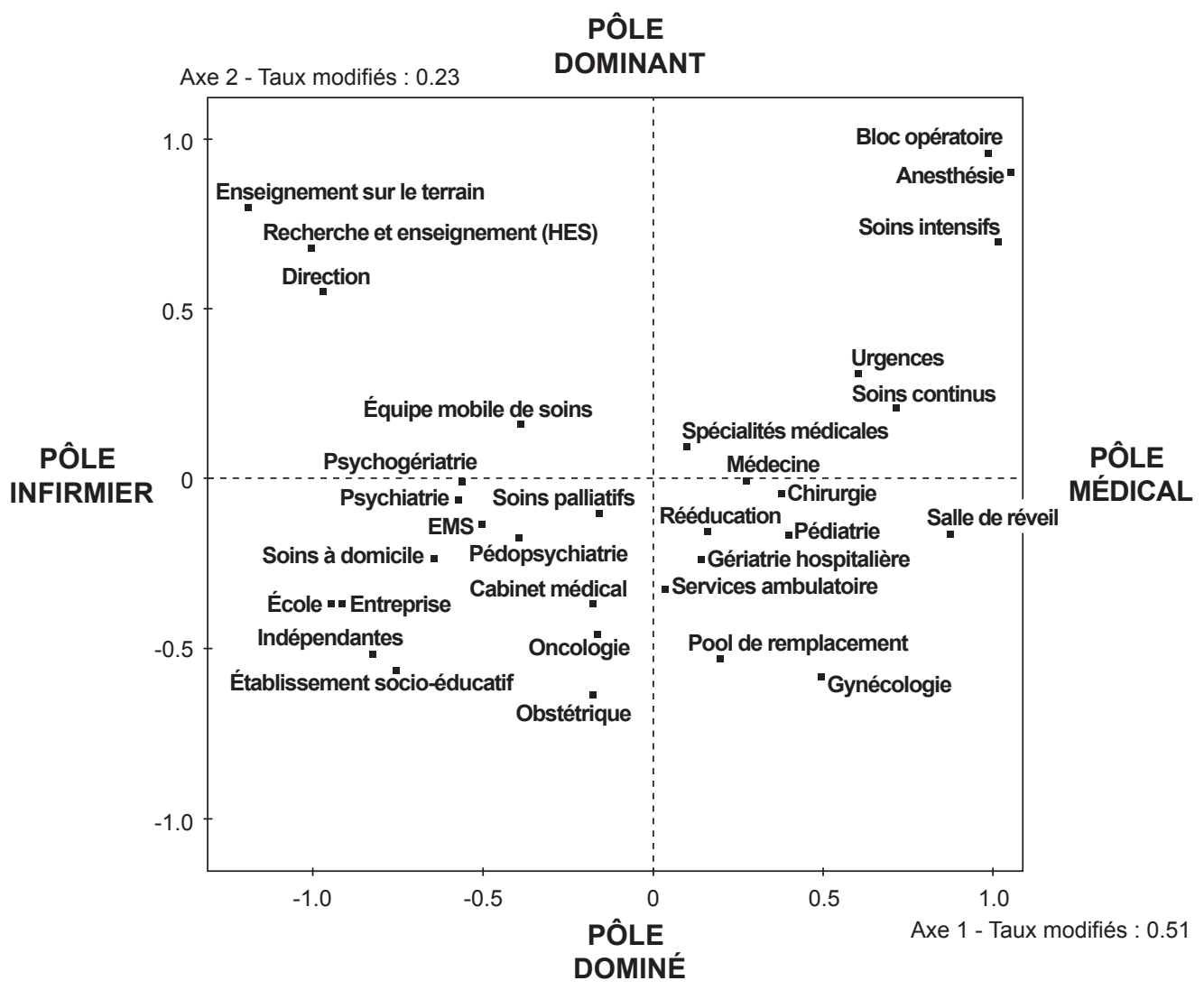

Source: EPRIS.

Note: Les secteurs d'activités ont été projetés en éléments supplémentaires. $\mathrm{N}=2923$.

La structure et le volume de capital sont fortement associés aux différents secteurs d'activité. Les services hospitaliers de soins aigus (bloc opératoire, anesthésie, soins intensifs, urgences) se situent du côté des infirmières présentant un volume élevé de capital à dominante médicale (en haut à droite); les services hospitaliers de soins aux personnes âgées (gériatrie hospitalière), 
aux femmes (gynécologie) ou aux enfants (pédiatrie) se situent du côté des infirmières présentant un volume faible à dominante médicale; les secteurs éloignés de la pratique clinique (recherche et enseignement, direction) se situent du côté des infirmières présentant un volume élevé à dominante infirmière; et les secteurs extrahospitaliers de soins aux personnes âgées (EMS, soins à domicile) et de santé communautaire (santé scolaire, entreprise, établissements socio-éducatifs) se situent du côté des infirmières présentant un volume faible à dominante infirmière.

Nos résultats nous permettent ainsi d'esquisser les contours d'un espace infirmier dans lequel chaque infirmière occupe une position spécifique suivant le volume et la structure des capitaux médical et infirmier en sa possession. Les différentes positions repérables sont étroitement associées aux secteurs d'activité qui composent la profession. Des secteurs qui, comme nous le verrons dans le chapitre suivant, sont hiérarchisés aux yeux mêmes des infirmières selon des critères proprement médicaux. 


\section{Chapitre 4}

\section{Le prestige des secteurs d'activité}

Nous venons de voir que chaque secteur d'activité occupe une position spécifique au sein de l'espace infirmier. Ces positions sont définies de manière différenciée, mais aussi hiérarchisées. Il s'agit à présent de se demander dans quelle mesure les infirmières perçoivent cette hiérarchie des secteurs, en retenant comme indicateur le prestige qu'elles attribuent à chacun d'entre eux.

Pour ce faire, une liste de 20 secteurs était proposée dans le questionnaire. Les infirmières étaient invitées à attribuer à chacun un score de prestige compris entre 0 (pas du tout prestigieux) et 6 (très prestigieux). Le score moyen récolté par chaque secteur fait apparaître d'importantes disparités (graphique 4).

Alors que les secteurs dits "aigus» (soins intensifs, urgences, anesthésie) occupent le sommet de la hiérarchie de prestige, les secteurs liés aux soins chroniques (EMS, psychogériatrie) et à la santé communautaire (santé scolaire, milieu socio-éducatif, milieu carcéral) sont relégués à sa base. Quant aux secteurs hospitaliers «semi-aigus» (chirurgie, obstétrique, médecine), ils occupent une position intermédiaire. 
Graphique 4. Niveau de prestige des secteurs ${ }^{29}$

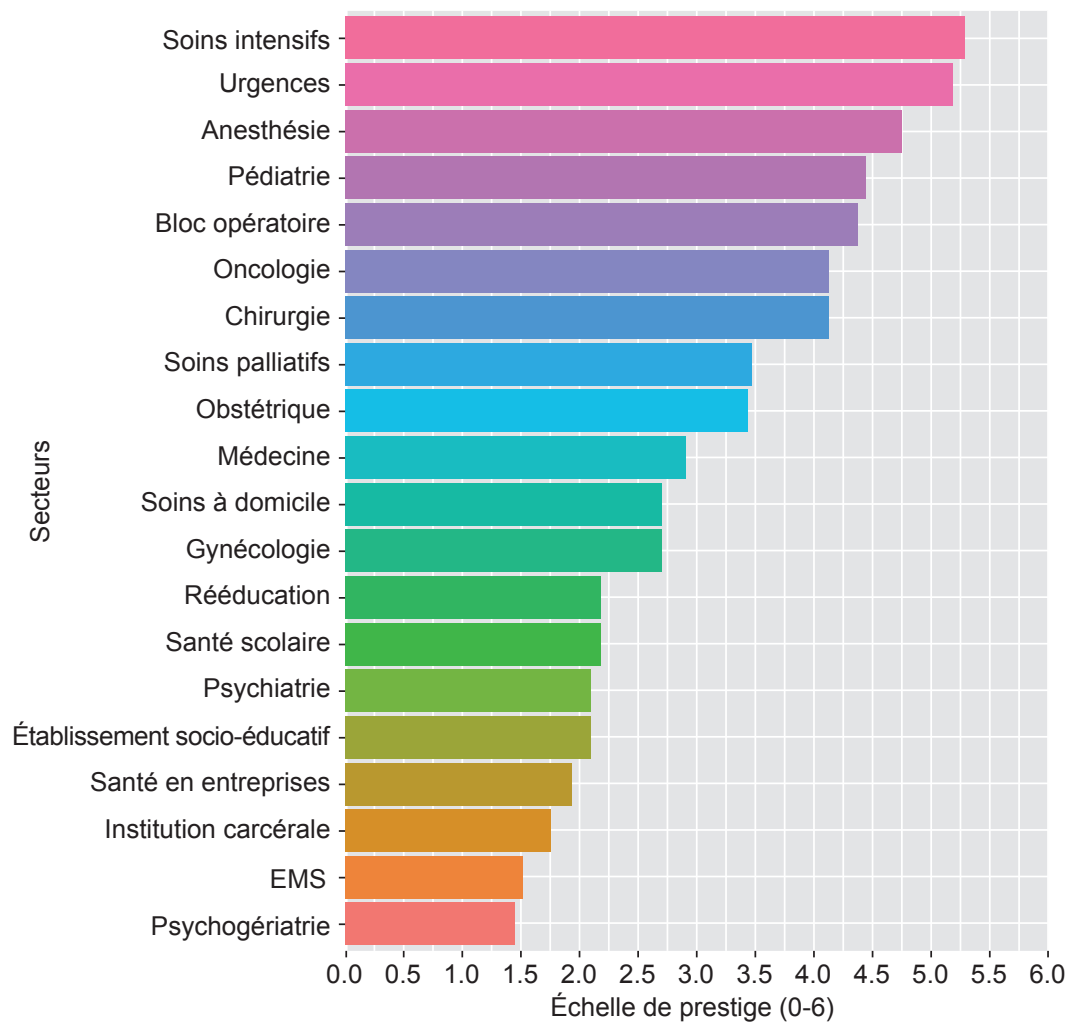

Source : EPRIS.

Note : Scores moyens sur l'échelle de prestige (0 à 6) attribués à 20 secteurs.

Cette échelle de prestige est fortement intériorisée par les infirmières des différentes fractions. Exerçant en soins intensifs, Madame Jacques ${ }^{30}$ estime qu'elle et ses collègues ne sont "pas meilleures que les autres, mais plus à la pointe que les autres». Infirmier

${ }^{29}$ Une première version de cette échelle de prestige a été publiée par LoNGCHAMP, Toffel, Bühlmann \& Tawfik (2016). Certains développements de l'analyse sont également présentés dans Toffel (2018).

${ }^{30}$ Afin de respecter l'anonymat des infirmières, les noms ont été modifiés. 
anesthésiste, Monsieur Bideau a le sentiment d'être mis «sur un piédestal» par les infirmières «des étages» (c'est-à-dire principalement des secteurs de médecine et de chirurgie) qui font appel à ses services:

"C'est idéalisé, parce que, par exemple, aux étages, quand ils arrivent pas à piquer, ils nous appellent nous. Alors on est... C'est vrai que nous... C'est une profession qui est euh... qui est assez mise sur un piédestal j'ai l'impression.»

Une relation peut être établie entre cette échelle de prestige et l'espace des positions que nous avons exposé précédemment (graphique 3). On constate en effet que les secteurs les plus prestigieux sont ceux où exercent les infirmières à fort volume de capital à composante médicale (en haut à droite), alors que les secteurs les moins prestigieux sont ceux où exercent les infirmières à faible volume de capital à composante infirmière (en bas à gauche). Les secteurs moyennement prestigieux étant ceux où exercent les infirmières à faible volume de capital à composante médicale (en bas à droite).

Deux questions se posent face au constat d'une telle échelle de prestige. Premièrement, quelle est la logique sous-jacente à ce classement? Plus précisément, retrouve-t-on ici le même raisonnement que celui que l'on observe dans les études portant sur le prestige des différentes professions? Deuxièmement, toutes les infirmières s'accordent-elles sur cette échelle de prestige? Ou observe-t-on au contraire des variations suivant leur âge, leur sexe ou encore le secteur dans lequel elles exercent?

\section{Le prestige des secteurs infirmiers selon certaines caractéristiques classiques}

La question du prestige des professions a été largement étudiée en sociologie. Dans son étude désormais classique, Donald Treiman (1977) montre que le prestige d'une profession est fortement 
corrélé à son revenu moyen ainsi qu'au niveau de formation de ses occupants. Ayant davantage d'indicateurs à disposition, mais pour la seule situation française, Christine Chambaz, Éric Maurin et Constance Torelli (1998) montrent que la situation contractuelle est la dimension la plus fortement associée au prestige d'une profession, qui varie en raison directe du salaire moyen et $\mathrm{du}$ taux d'emplois dans le secteur public. Les qualifications des individus jouent également un rôle, puisque les professions les plus prestigieuses sont celles où le niveau de formation moyen est le plus élevé. L'autonomie intervient aussi, le prestige d'une profession étant d'autant plus élevé que les consignes de travail y sont moins nombreuses et que la possibilité de choisir l'organisation et les méthodes de travail y est importante. Quant à l'autorité, elle joue un rôle négatif, une profession étant d'autant moins prestigieuse qu'elle implique des fortes responsabilités d'encadrement.

Afin de vérifier si les caractéristiques déterminant le prestige des professions produisent le même effet clivant sur les différents secteurs infirmiers, nous avons mené une analyse statistique comprenant les variables classiques mobilisées dans les études sur le prestige des professions (voir tableau A6 en annexe 3). Soit, pour chaque secteur d'activité, la stabilité de la situation contractuelle et du revenu (mesurée ici par l'ancienneté et le pourcentage de postes dans le service public, ainsi que par le revenu), les qualifications personnelles des individus (mesurées ici par la proportion d'individus ayant suivi une formation après la formation de base et leur âge), l'autonomie et l'indépendance dans le travail (mesurées ici par la part des tâches médicodéléguées) et, enfin, l'autorité dont jouissent les individus (mesurée ici par la proportion de cadres) ${ }^{31}$.

Les résultats montrent que la plupart de ces déterminants n'exercent pas d'effets significatifs sur le prestige des secteurs.

\footnotetext{
${ }^{31}$ Les résultats sont issus d'une régression linéaire menée sur les données agrégées, les 20 secteurs analysés ici constituant les «individus» de la régression.
} 
Ainsi, et alors que la situation contractuelle est la dimension la plus fortement associée au prestige des professions, il s'agit précisément de la dimension qui entretient le lien le plus faible avec le prestige des secteurs infirmiers: l'ancienneté, le taux de postes dans le secteur public et le revenu demeurent ainsi sans effet. S'agissant des qualifications personnelles, la proportion d'individus ayant suivi une formation après la formation de base n'est pas non plus significativement associée au prestige des secteurs. Concernant l'autorité, elle est significativement associée au prestige et indique une tendance semblable à ce qui s'observe dans les recherches portant sur les professions: les responsabilités d'encadrement sont négativement associées au prestige. La proportion de cadres est en effet particulièrement élevée parmi les secteurs les moins prestigieux (48\% en EMS, $43 \%$ en psychogériatrie), alors qu'elle est particulièrement basse parmi les secteurs les plus prestigieux (13\% aux urgences, $7 \%$ aux soins intensifs et en pédiatrie).

Parmi les dimensions habituellement retenues pour évaluer le prestige des professions, l'autonomie est finalement la seule qui soit significativement associée au prestige des secteurs. Mais dans un sens inverse à celui attendu : c'est en effet l'absence d'autonomie qui est associée au prestige des secteurs infirmiers. Les services les plus prestigieux sont ainsi ceux qui présentent l'indice de tâches médico-déléguées le plus élevé. Sur une échelle de 0 à 3 , on trouve des moyennes de 2,8 en anesthésie, 2,6 aux soins intensifs ainsi qu'aux urgences, et 2,3 en pédiatrie. À l'inverse, les moyennes les plus faibles se retrouvent parmi les secteurs les moins prestigieux: 1,9 en psychogériatrie et en EMS.

La logique qui sous-tend le prestige des secteurs infirmiers ne correspond donc pas à celle qui sous-tend le prestige des professions en général. La relation qui unit l'autonomie au prestige des secteurs est non seulement la seule significative de toutes celles observées jusqu'ici, mais aussi la plus opposée à celle que l'on observe parmi les professions en général. Les secteurs les plus prestigieux sont ainsi ceux où les tâches médico-déléguées sont les 
plus nombreuses - soit ceux où la possibilité de développer un «rôle propre» infirmier est la plus faible.

\section{Le prestige des secteurs infirmiers selon certaines caractéristiques spécifiques}

Afin de mieux cerner la logique sous-jacente au prestige des secteurs infirmiers, nous avons intégré dans l'analyse trois propriétés plus spécifiques à la profession infirmière (voir tableau A7 en annexe 3). Premièrement, un indice de soins relationnels, qui synthétise les réponses à 5 questions sur l'importance de la relation entre les infirmières et les patients. Un score élevé sur l'indice de soins relationnels renvoie à une forte autonomie professionnelle, cette dimension des soins ne faisant jamais l'objet d'une délégation médicale et étant, de ce fait, considérée comme partie intégrante $\mathrm{du}$ «rôle propre» infirmier. Deuxièmement, un indice de soins de confort et d'hygiène, qui synthétise la fréquence à laquelle les infirmières prodiguent des soins tels que déplacer le patient, l'aider à se mobiliser, l'accompagner aux toilettes, le laver, l'aider à s'alimenter, etc. Ces soins recouvrent une connotation très ambivalente dans la profession puisqu'ils sont à la fois considérés comme le «sale boulot» (Hughes, 1996) - qui peut être délégué aux subalternes - et comme le "cœur du métier» - le contact direct avec le corps du patient, moment privilégié de la "relation", étant ce qui marque la différence entre professions infirmière et médicale. Enfin et troisièmement, un indice médico-technique, qui synthétise la fréquence des contacts (directs, par téléphone, mail, fax, etc.) entre infirmières et médecins d'une part, et la fréquence d'utilisation des 8 accessoires les plus courants de la profession d'autre part (appareils de mesure, ordinateur, matériel d'aide à la respiration, défibrillateur, scope, ECG, seringue et matériel pour un pansement). Tout comme pour celui des tâches médico-déléguées, un score élevé sur cet indice renvoie à une faible autonomie professionnelle. 
Alors que l'indice des soins de confort et d'hygiène n'est associé au prestige dans aucun des modèles, l'indice de soins relationnels présente une association négative avec le prestige dans les deux premiers modèles, confirmant ainsi que la forte autonomie d'un secteur à l'égard du corps médical se traduit par son mauvais classement dans la hiérarchie de prestige. Mais c'est assurément l'indice médico-technique qui présente l'association la plus forte avec le prestige des secteurs, puisque son introduction dans le troisième modèle annule l'effet de l'indice de soins relationnels. Sur une échelle allant de 0 à 5, les secteurs les plus prestigieux se distinguent ainsi par des moyennes beaucoup plus élevées (3,8 en anesthésie, 3,5 aux urgences et aux soins intensifs, 3,3 au bloc opératoire) que les secteurs les moins prestigieux (0,9 en entreprise et en EMS, 0,8 en établissement socio-éducatif, 0,6 dans la santé scolaire). Un résultat qui confirme pleinement que la dimension médicotechnique - qui s'accompagne d'une faible autonomie des infirmières - constitue la caractéristique la plus fortement associée au prestige d'un secteur.

On retiendra de cette analyse que le prestige des secteurs infirmiers est avant tout déterminé par leur dimension médicotechnique. L'effet de cette dimension est tel que, contrairement à ce qui s'observe pour les professions en général, ça n'est pas l'autonomie, mais bien son absence qui est associée au prestige des secteurs de l'espace infirmier. Si bien que l'on peut qualifier la hiérarchie de prestige à laquelle adhèrent les infirmières de "prestige par procuration».

\section{L'échelle de prestige : un consensus absolu entre les infirmières}

Jusqu'à présent, nous avons fait comme si toutes les infirmières s'accordaient sur les classements de prestige des secteurs, quels que soient leur âge, leur sexe, leur nationalité 
ou encore leur secteur d'activité. Mais est-ce vraiment le cas? Afin de répondre à cette question, nous suivrons la procédure méthodologique classique initiée par Treiman (1977) et poursuivie par Chambaz et ses collègues (1998). Il s'agira donc de considérer les scores moyens de prestige que des groupes d'individus définis par certaines propriétés attribuent à chaque secteur. Nous retiendrons quatre propriétés individuelles: le sexe, l'âge, la nationalité et le secteur d'activité.

La corrélation entre les échelles de prestige respectives des femmes et des hommes s'élève à 0,995 , celle entre les échelles des infirmières les plus âgées et les plus jeunes à 0,992 et celle entre les échelles des infirmières suisses et étrangères à 0,991. Quant à la moyenne des corrélations entre les échelles des infirmières exerçant dans les 20 secteurs dont il est question ici, elle s'élève à 0,969 . C'est donc une seule et même échelle de prestige qui a été intériorisée par toutes les infirmières, et ce quels que soient leur sexe, leur âge, leur nationalité ou leur lieu d'exercice. Autrement dit, une infirmière de 25 ans, de nationalité étrangère et exerçant aux soins intensifs proposera la même échelle de prestige des secteurs qu'un homme de 50 ans, de nationalité suisse et exerçant en EMS. Ces résultats rejoignent ceux de Treiman (1977), qui a montré que les hiérarchies de prestige des professions sont à peu près invariantes à travers le temps et l'espace, et a par la suite confirmé ces résultats en incluant un nombre très élevé de professions dans ses analyses (GANZEBoom \& Treiman, 1996). Michael Hout et Thomas DiPrete (2006) parlent à ce propos de "constante de Treiman", et vont même jusqu'à considérer qu'il s'agit là du seul fait universel jamais découvert par la recherche sociologique.

Comme le montrent Clément Bastien et Olivia Rick (2009), la hiérarchisation des secteurs infirmiers est intériorisée dès les études en soins qui contribuent à situer les «soins aigus» au sommet de la hiérarchie tout en reléguant à sa base les secteurs de "médicalisation minimale» que sont par exemple les soins aux personnes âgées. Les entretiens que nous avons menés avec 
les infirmières montrent ainsi que l'ordre chronologique des stages durant les études peut être interprété comme une forme d'objectivation de cette hiérarchie:

"J'ai commencé par la gériatrie. C'était souvent les premiers stages où on débutait parce que c'était une approche, au niveau de la personne âgée, je pense que c'était plus simple que de commencer à demander un service un peu plus aigu." Mme Novak, urgences

À l'inverse, les soins intensifs jouissent d'une grande popularité auprès des étudiantes et étudiants. Se remémorant ses études, Madame Jacques déclare ainsi que "les soins intensifs, c'était LE stage que tout le monde voulait».

Alors que leur attirance pour les soins intensifs se renforce au cours de leur cursus de formation, les étudiantes et étudiants se montrent au contraire toujours plus réticents à travailler auprès de personnes âgées. Une réticence qui n'est pas à mettre sur le compte d'une méconnaissance de leur part, puisqu'elle se manifeste en particulier chez les étudiantes et étudiants qui ont effectué le plus de jours de stages auprès des personnes âgées (Stevens, 2011).

Le consensus qui unit les infirmières dès lors qu'il s'agit de classer les différents secteurs d'activité sur une échelle de prestige est convergent avec ce qui s'observe dans les études sur les professions. Nos résultats relèvent cependant une apparente contradiction: contrairement aux professions les plus prestigieuses, les secteurs les plus prestigieux ne se distinguent ni par l'ancienneté des infirmières qui y exercent, ni par leur formation, ni par leur revenu, ni par leur statut. Ces infirmières semblent même moins bien loties que celles qui exercent dans les secteurs les moins prestigieux, puisqu'elles bénéficient d'une moindre autonomie dans leur travail. S'il en va ainsi, c'est parce 
que la hiérarchie de prestige intériorisée par les infirmières ne correspond pas à une logique proprement infirmière, mais bien à une logique médicale: ce sont les secteurs les plus médicalisés et les plus technicisés qui sont considérés comme les plus prestigieux. Le prestige - bien réel - que retirent les infirmières qui y exercent peut donc être considéré comme un "prestige par procuration", ce qui est symptomatique de l'ascendant qu'exerce le champ médical sur l'espace infirmier. 


\section{Partie II \\ Visions et divisions}

En prenant appui sur la théorie des champs, nous avons présenté dans la partie précédente la profession infirmière comme un espace de positions différenciées. Il s'agit à présent d'approfondir l'analyse de la différenciation interne de la profession en montrant que les différentes positions que l'on y repère sont associées à des représentations et pratiques spécifiques.

On peut distinguer quatre fractions d'individus au sein de la profession infirmière: les "dominantes médicales", les "dominées médicales», les "élites infirmières» et les "hétérodoxes" (graphique 5) ${ }^{32}$. L'identification de ces quatre fractions résulte d'une analyse de classification, méthode qui suggère des regroupements d'individus en fonction de leur positionnement sur les deux axes factoriels retenus dans l'ACM présentée au chapitre 3, soit selon la structure et le volume de leurs capitaux médical et infirmier.

${ }^{32}$ La description de ces quatre fractions a fait l'objet de travaux antérieurs (Longchamp, Toffel, Bühlmann \& TaWfik, 2018; Toffel, 2018). 
Graphique 5. Quatre fractions d'infirmières ${ }^{33}$

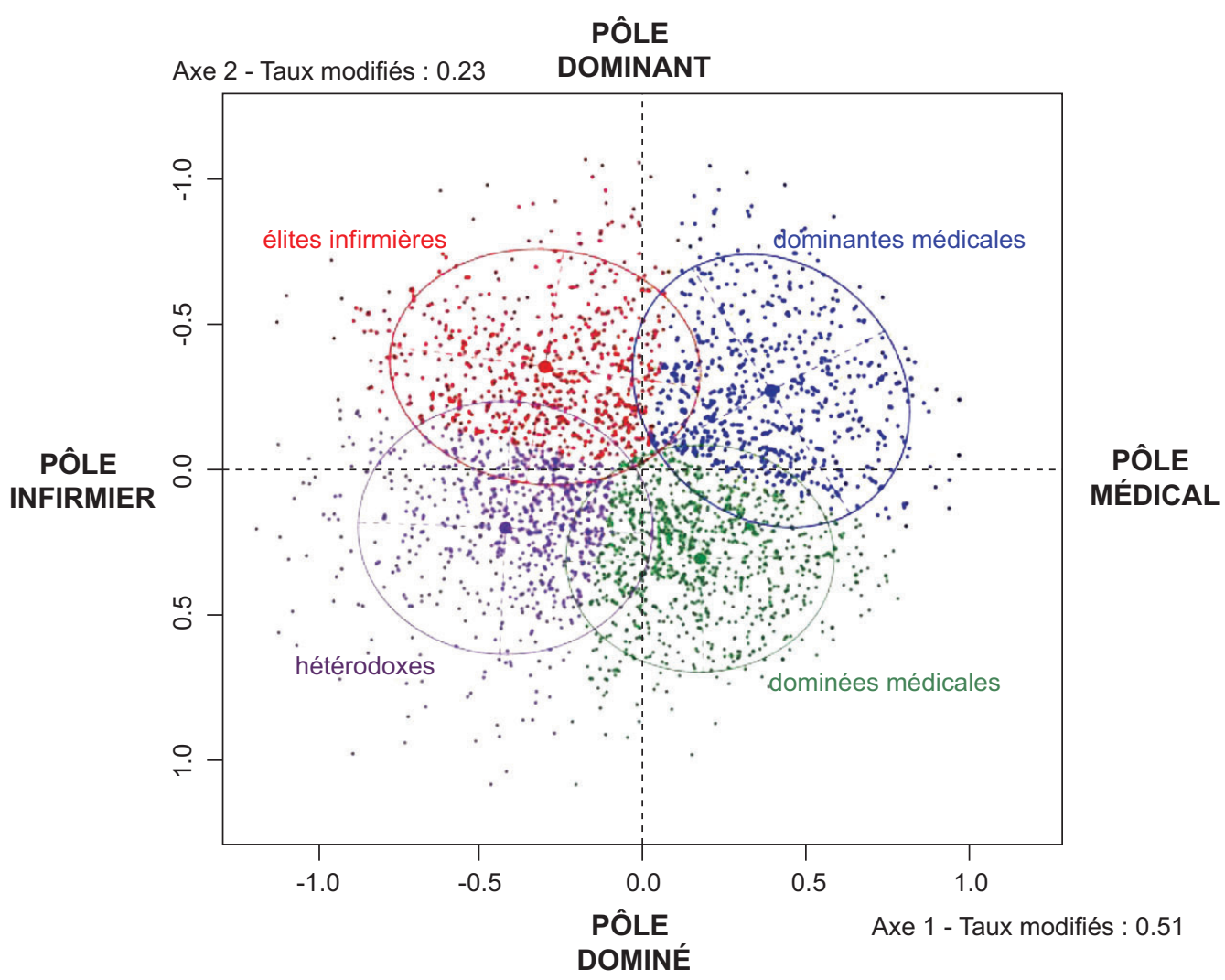

Source: EPRIS.

Note: Les effectifs des fractions dans notre échantillon sont les suivants: 654 dominantes médicales, 922 dominées médicales, 680 hétérodoxes, 667 élites infirmières. $\mathrm{N}=2923$.

${ }^{33}$ Partition issue d'une analyse de classification mixte sur le plan factoriel 1-2 de l'ACM. Les quatre fractions obtenues ont été caractérisées par les modalités actives, complétées par des modalités supplémentaires (voir tableaux A12 et A13 en annexe 4). Seules les modalités dont la valeur test est égale ou supérieure à 2 ont été retenues pour la caractérisation des classes. Chaque ellipse de concentration possède deux écarts-types sur chaque axe (les écarts-types n'étant pas les mêmes sur chacun des deux axes) et contient 86,5\% des individus de la fraction concernée (Le Roux \& Rouanet, 2010). 
Chacune de ces fractions se distingue par des prises de position spécifiques, tant en ce qui concerne la définition du métier qu'en ce qui concerne le type de relations entretenues avec les autres professionnels de santé et les patients. Observons que, en dépit de l'impression visuelle offerte par les ellipses du graphique, les frontières entre les fractions ne sont pas nettes: comme le montre le nuage des individus, c'est au contraire à un continuum que nous avons affaire, une part importante des infirmières se situant à la frontière entre les différentes fractions. C'est ainsi, tout en gardant à l'esprit que ces résultats révèlent des tendances et non des réalités figées et opposées, que l'on peut décrire les caractéristiques typiques de ces quatre fractions infirmières.

Si la "photographie» est une métaphore utile pour rendre compte du concept d'espace social, ce dernier ne doit toutefois pas être considéré comme une réalité figée. Parce qu'il est un espace de luttes, un espace social est nécessairement dynamique. Les quatre fractions qui composent l'espace infirmier se caractérisent ainsi non seulement par des positions spécifiques, mais aussi par leur dynamique croissante ou décroissante. Nous verrons ainsi que les deux fractions qui se trouvent au pôle infirmier de l'espace (les «élites infirmières» et les "hétérodoxes») se trouvent à la croisée des chemins, la première étant en pleine croissance, alors que la seconde semble plutôt sur le déclin. À cette dynamique liée à des destins historiques et collectifs s'ajoute une seconde dynamique, liée à des trajectoires individuelles: loin de n'occuper qu'une seule position au cours de leur carrière, les infirmières présentent au contraire une forte propension à se déplacer au sein de l'espace infirmier. S’il existe une trajectoire modale allant du pôle médical (en première partie de carrière) au pôle infirmier (en seconde partie de carrière), d'autres trajectoires sont possibles, chacune étant associée à des caractéristiques individuelles spécifiques (chapitre 9). 



\section{Chapitre 5}

\section{Les dominantes médicales}

Intéressons-nous plus en détail à ces quatre fractions d'infirmières qui composent l'espace infirmier. La première se situe en haut à droite de l'espace (graphique 5). Ces infirmières sont largement surreprésentées aux soins intensifs (elles y représentent $17,4 \%$ des répondantes, contre $5,7 \%$ de l'échantillon complet), au bloc opératoire ( 9,8 vs $2,9 \%)$, aux urgences $(9,3$ vs $4,8 \%$ ) et aux soins continus ( 5,2 vs $2,4 \%$ ), soit les secteurs où la médicalisation et l'appareillage sont les plus élevés. La hiérarchisation des soins et des soignantes étant organisée "en fonction de leur proximité réelle ou supposée avec des soins médicaux» (VÉGA, 2000, p. 15), ces secteurs sont par ailleurs unanimement considérés au sein de la profession comme étant les plus prestigieux (voir chapitre 4). Ces infirmières se caractérisent par une très forte mobilisation de leurs connaissances en physiopathologie $(73,3 \text { vs } 30,1 \%)^{34}$ et en utilisation de

\footnotetext{
${ }^{34}$ Sauf autre précision, les deux chiffres entre parenthèses renvoient respectivement au taux de réponse de la fraction considérée sur la modalité la plus favorable, comparé au taux de réponse de l'ensemble de l'échantillon sur cette même modalité. Dans le cas présent, les infirmières de la fraction "dominantes médicales» sont $73,3 \%$ à déclarer mobiliser "toujours" leurs connaissances en physiopathologie, contre $30,1 \%$ des infirmières de l'ensemble de l'échantillon. Avantageux car synthétique, ce mode de présentation ne rend cependant pas compte des contrastes beaucoup plus
} 
machines (77,2 vs 27,5\%), par un taux d'activité élevé (62,6\% exercent à plus de $80 \%$, contre $44,5 \%$ de l'échantillon total) et par des formations longues $(59,2$ vs $55,9 \%)$ à orientation très médicale $(65,5$ vs $24,6 \%)$. Elles mobilisent en revanche moins fréquemment leurs valeurs personnelles (27,9 vs 36,4\%), leurs compétences relationnelles $(69,0$ vs $74,2 \%)$ et leurs connaissances en modèles infirmiers $(50,6 \%$ d'entre elles les mobilisent rarement, contre $43,3 \%$ de l'échantillon). Elles se distinguent, enfin, par un rejet des caractéristiques typiquement associées au capital infirmier, telles que l'assistance à des congrès de soins (34,1 vs $41 \%)$, de développement personnel $(2,6$ vs $13,4 \%)$ ou de médecine complémentaire $(0,3$ vs $5,4 \%)$, l'affiliation à l'ASI (16,8 vs $33,3 \%)$ ou encore l'ouverture à des soins non conventionnels (0,3 vs 7,5\%). Cela témoigne non seulement d'un capital infirmier relativement faible, mais aussi d'une forte orthodoxie médicale.

En se référant aux deux dimensions de l'espace infirmier présentées au chapitre 3, l'on constate que ces infirmières associent un volume élevé de capital à une structure à dominante médicale. Le prestige dont elles jouissent au sein de l'espace infirmier est typiquement un prestige par procuration (cf. chapitre 4) qui leur est en grande partie conféré par leurs compétences médicotechniques et leur proximité avec la profession médicale. Les infirmières appartenant à cette fraction peuvent ainsi être qualifiées de «dominantes médicales».

\section{Des secteurs de référence}

Les «soins aigus» occupent une place à part dans l'espace infirmier. Tout se passe comme si ces services représentaient la référence par rapport à laquelle les professionnelles tendent

importants qui distinguent les quatre fractions d'infirmières. Pour se faire une idée plus précise, le lecteur pourra se référer aux résultats complets qui figurent en annexe 4 dans les tableaux A12 (pour les modalités actives) et A13 (pour les modalités supplémentaires). 
toujours à se situer. Et le fait que cette reconnaissance se manifeste dans l'ensemble de l'espace infirmier constitue un indice $\mathrm{du}$ caractère dominant du capital médical. Cette domination peut encore se repérer sur la base de multiples indices, à commencer par l'acquisition d'une certaine expérience qui donne accès aux secteurs les plus médicalisés (soins intensifs, anesthésie et urgences), condition d'entrée parfois assortie d'une expérience professionnelle demandée dans ces services:

"- Pour nous, pour les soins anesthésistes, c'est deux ans [de pratique] [...] on demande deux ans de soins aigus.

- De soins aigus, carrément?

- Pour nous, soins aigus, c'est la médecine à l'hôpital, par exemple la chirurgie; mais contrairement à la gériatrie, ou bien les soins palliatifs ou le... ou dans un $C M S^{35}$; il faut un peu de technique quoi.» M. Bideau, anesthésie

Une fois entrées dans ces services, les infirmières suivent souvent des formations assez longues (environ deux ans) en cours d'emploi, afin d'acquérir un diplôme assorti de compétences spécifiques à ces secteurs d'activité. Si ces formations ne sont pas toujours obligatoires, elles sont souvent souhaitées par les institutions. Formations "certifiantes", elles permettent aux infirmières qui en bénéficient d'accéder à un nouveau statut généralement sanctionné par une petite hausse salariale. Cette hausse demeure cependant modeste, et le revenu équivalent plein temps des dominantes médicales, qui s'élève à 6314 francs, les situe légèrement en dessous de la moyenne des revenus $(6401 \text { francs })^{36}$.

Si la formation spécialisée est très importante, c'est surtout l'expérience que confère l'exercice dans un secteur de soins aigus

\footnotetext{
35 Centre Médico-Social.

${ }^{36}$ Les revenus mentionnés ici sont valables pour une femme âgée de 43 ans (âge moyen de notre échantillon). Pour les revenus moyens des différentes fractions non contrôlés par d'autres variables, voir tableau A4 en annexe 3.
} 
qui semble faire de l'infirmière une «bonne professionnelle», et ce quelle que soit la suite de sa carrière. C'est que le passage par l'hôpital a une forte influence sur les schèmes de perception. Les discours que tiennent les infirmières les plus fortement dotées de capital médical sur leurs trajectoires professionnelles sont ainsi ponctués d'expressions telles que "progresser» ou «aller plus loin», autant de termes qui révèlent la présence d'une forte disposition promotionnelle. Infirmier anesthésiste, Monsieur Bideau se présente ainsi comme ayant toujours eu "un plan de carrière assez bien défini». Dans la même veine, Madame Jacques, infirmière en soins intensifs, présente sa trajectoire comme une ascension: "Ça suit un degré de complexité en fait."

Une telle verticalisation des trajectoires se traduit par un climat très compétitif dans lequel le sentiment que les collègues souhaitent progresser hiérarchiquement est plus répandu qu'ailleurs $(6,8$ vs $4,6 \%)$. De fait, cette hiérarchisation se manifeste d'abord à l'intérieur des secteurs, où elle apparaît comme principe de distinction entre les pairs. Le cumul de plusieurs années de pratique à temps plein dans des services «aigus » apparaît comme une garantie d'accumulation de capital médical permettant aux infirmières concernées de marquer leur différence à l'égard de celles qui, moins expérimentées, n'exercent de surcroît qu'à temps partiel. La hiérarchisation se révèle encore dans l'énumération des critères qui président à l'attribution des patients lors d'une prise en charge. Les patients étant situés sur une échelle allant du simple au complexe, ils sont attribués aux infirmières en fonction de leur degré d'accumulation de capital médical, comme le révèle Madame Jacques, infirmière aux soins intensifs :

"On a des jeunes collègues qui viennent d'arriver, ça fait peut-être six mois, une année. On essaie de leur donner des patients pas trop complexes."

Mais si cette hiérarchisation se manifeste à l'intérieur des différents secteurs, elle se manifeste aussi, et peut-être surtout, 
à l'échelle de l'espace infirmier dans son ensemble. Il se dégage en effet, dans les discours des dominantes médicales, une nette hiérarchisation des différents secteurs d'activité. Évoquant le souvenir de sa perception des infirmières de soins intensifs alors qu'elle était étudiante, Madame Jacques se remémore «des infirmières... pas meilleures que les autres, mais plus à la pointe que les autres». Ayant désormais intégré cette "élite professionnelle", c'est ce même regard venu des autres services qu'elle semble percevoir:

"On a un peu la réputation qu'on se la pète, qu'on se sent meilleures que les autres parce qu'on travaille dans un service plus à la pointe. Donc qu'on se croit meilleures, plus performantes.»

Réelle ou imaginaire, une telle situation ne manque pas de susciter un sentiment d'élection voire d'exceptionnalité professionnelle. Seul étudiant de sa volée à avoir accédé à un service d'urgences comme premier poste après ses études, Monsieur Ropars n’hésite pas à associer cela à ses qualités personnelles: «Mon côté battant et volontaire, motivé, a fait que j'ai pu m'en sortir aux urgences.» Ces dispositions dominantes se manifestent par un très fort sentiment d'employabilité (17,8 vs $12,9 \%$ ) qui, ignorant la diversité des compétences attendues dans les différents secteurs d'activité de la profession, tend à universaliser la valeur des compétences associées au seul capital médical. Monsieur Ropars estime ainsi que son potentiel d'employabilité lui permettrait de postuler dans n'importe quel service: "Je peux aller postuler en médecine, en chir., où je veux!" Â l'inverse, il relègue les infirmières issues du secteur gériatrique au plus bas de l'échelle d'employabilité.

\section{La volatilité du capital médical}

Mais à ce sentiment d'exceptionnalité répond une certaine crainte de perdre les acquis. Il apparaît en effet que le capital médical se caractérise par une certaine volatilité qui peut entrâner un sentiment d'insécurité lié à sa perte potentielle et 
au déclassement professionnel qui s'en suivrait. Cette volatilité se révèle notamment chez les infirmières qui, exerçant dans un secteur peu médicalisé, se voient ponctuellement sollicitées pour intervenir dans un secteur très médicalisé. Exerçant en néonatologie, Madame Ortiz appréhende les moments où elle est appelée aux soins intensifs:

"Ça fait six ans que je ne suis pas retournée aux soins intensifs. Je vais aider ponctuellement quand même, mais c'est un gros stress [...]. Les vieux disent que c'est comme le vélo: ça revient. Mais non, c'est pas vrai. [...] J'ai beaucoup de collègues qui sont ici et qui craignent de devoir retourner de l'autre côté pour de l'aide. [...] On appréhende un peu tous.»

La crainte face à la volatilité du capital médical se retrouve également parmi les infirmières qui exercent à temps partiel. On retrouve même parfois ici un processus d'auto-exclusion, comme dans le cas de Madame Ruffenach travaillant dans des secteurs de chirurgie et soins continus et qui, suite à la réduction de son taux d'activité consécutive à la naissance de son premier enfant, a préféré renoncer à son poste de responsable des lits de soins continus :

"J'ai fondé une famille. Donc j'ai décidé de baisser mon temps de travail et de quitter ce poste-là. Parce que je trouvais pas judicieux, en travaillant à un petit pourcentage, de garder ce poste-là.»

On le voit, l'enjeu consiste ici à rester en contact étroit avec les secteurs les plus «aigus», et ce pour deux raisons. Exercer et maintenir ce que l'on pourrait qualifier de "tour de main» d'une part, se tenir informée des rapides évolutions technologiques d'autre part $^{37}$. C'est dire que la crainte d'une perte de capital

${ }^{37}$ Les évolutions technologiques expliquent l'abandon, par certains hôpitaux, du statut de veilleuse de nuit. Échappant aux formations dispensées durant 
médical ne s'exerce que très inégalement selon le sexe. Alors que les femmes y sont très exposées sitôt la naissance de leur premier enfant, et se voient souvent contraintes de quitter l'hôpital, soit pour exercer - souvent à taux partiel - dans d'autres secteurs, soit pour délaisser la profession, les hommes demeurent largement épargnés. Pour ces derniers, la sortie de l'hôpital peut être plus facilement repoussée en "fin de carrière» comme le dit Monsieur Pichoud:

"L'extra hospitalier? [...] Je me suis dit: "Ben, c'est quelque chose que je garde volontiers pour [...] la fin de carrière". Parce que c'est toujours cette peur, enfin peur entre guillemets; je pense que celui qui veut vraiment revenir à l'hôpital, qui fait ses preuves, il trouve du travail. Mais il y a quand même... Je sais pas par quoi c'est vébiculé cette crainte de: "Si tu vas faire des soins à domicile, ça va être difficile un jour de reprendre à l'hôpital."”

Et le maintien d'une activité durable et à un taux élevé dans les services les plus "aigus " favorise sans doute l'accès aux postes de cadres et aux spécialités cliniques les plus masculinisées, telle l'anesthésie (Lindsay, 2007; Рicot, 2005).

\section{Une relative indifférenciation des tâches entre infirmières et médecins}

Ces dispositions promotionnelles s'accommodent sans peine de dispositions plutôt conservatrices dès lors qu'est abordée la question des relations entretenues avec les médecins. Relevons d'abord que les dominantes médicales sont de loin les infirmières

la journée, les infirmières exclusivement affectées aux horaires nocturnes sont en effet susceptibles de se trouver en difficulté lors de l'utilisation de nouveaux appareils. La dimension "technique» de certains services explique ainsi, pour partie au moins, la flexibilité des horaires à laquelle sont soumises les infirmières qui y exercent. 
qui entretiennent les interactions les plus fréquentes avec les médecins. Ainsi, et alors que la fréquence de ces interactions est de l'ordre de moins d'une fois par semaine en EMS et de quelques fois par jour en médecine, on passe à une fréquence d'une fois par heure en moyenne aux soins intensifs ${ }^{38}$. $\hat{A}$ cette caractéristique quantitative de la relation entre infirmières et médecins s'ajoute une caractéristique qualitative: les dominantes médicales se distinguent en effet par une forte adhésion à la proposition suivant laquelle l'infirmière est le «bras droit du médecin" (18,3 vs 13,5\%). À l'encontre d'une vision émancipatrice de la profession que l'on retrouve fréquemment dans les prises de position politiques des infirmières de l'ASI ou dans d'autres régions de l'espace infirmier, certaines dominantes médicales s'accommodent ainsi très bien d'une claire hiérarchisation des rôles dans laquelle l'infirmière est au service du médecin:

"Pour moi, j’ai toujours dit, le médecin c'est un peu le cerveau pensant, et l'infirmier c'est un peu les mains, celui qui va faire ce que lui demande le médecin sans avoir un rôle éthiquement d'exécutant, mais plus... Il va plus être manuel, plus faire, plus... que le médecin qui va être plutôt la tête pensante." M. Ropars, urgences

À cette vision hiérarchique que d'aucuns qualifieront de passéiste, d'autres préfèrent une vision se référant à la complémentarité des rôles. Et il s'avère en effet que la frontière nette entre le rôle des infirmières et celui des médecins - que l'on observe dans les régions les moins médicalisées de l'espace - tend à s'estomper à mesure que l'on se rapproche du pôle médical, et notamment des secteurs les plus aigus où l'on assiste à un tandem infirmière-médecin. Infirmier anesthésiste,

\footnotetext{
${ }^{38}$ La proposition était: "Dans le cadre de votre travail, à quelle fréquence avez-vous des contacts (directs, par téléphone, mail, fax, etc.) avec les professionnel(le)s suivant(e)s?» L'échelle de réponse était comprise entre 1 «jamais» et 6 « $+10 x /$ jour», recodée de 0 «jamais» à 5 «+10x/jour».
} 
Monsieur Bideau va même jusqu'à évoquer une division des tâches "implicite» où tout n'est "pas vraiment défini» et où "on s'empiète un peu sur les plate-bandes":

"Effectivement, c'est pas vraiment défini; donc des fois on s'empiète un peu sur les plate-bandes. [...] Alors c'est vrai que quand on a des gens en formation, si on en a de nombreux, par exemple quand les médecins-assistants arrivent, au début, [...] enfin ils doivent apprendre un peu des techniques et tout ça; alors ça fait qu'ils le font à notre place, si on veut. Donc nous, si on a deux patients, pis ben on dit: "Moi je fais la première voie veineuse, toi la deuxième." Ben là quand ils sont en formation, ils font les deux. Mais, je dirais que c'est plutôt... c'est implicite.»

Cette relative indifférenciation peut même aller jusqu'à une forme d'anticipation du diagnostic médical par les infirmières. On retrouve ici Monsieur Ropars, pour qui cet aspect du travail constitue l'un des principaux attraits de son activité:

"L'intérêt de la prise en charge aux urgences, c'est d'anticiper le médecin. C'est là que nous, entre guillemets, en tant qu'infirmier, on progresse... Parce que si, entre guillemets, on arrive nous à se faire une idée du diagnostic avant le médecin, on améliore notre prise en charge. Et c'est tout gagnant pour le patient. C'est pour ça qu'ils ne prennent pas de jeunes diplômés pour les urgences. Qu'ils prennent de la bouteille... [sourire] Il sera quand même meilleur en diagnostic primaire infirmier. "

Le «diagnostic primaire infirmier» dont parle ici Monsieur Ropars ne doit pas être confondu avec le «diagnostic infirmier» entendu comme rôle spécifique reposant sur un stock de connaissances et de compétences propres à la profession. Car c'est bien ici à un domaine de compétences cliniques de type médical que se réfère Monsieur Ropars. Et l'on comprend alors que, de ce point de vue, le travail de l'infirmière consiste pour partie à faire ce que ferait le médecin si l'infirmière n'était pas là. 
Cette relative indifférenciation des rôles renvoie typiquement à la situation décrite par Elisa Giulia Liberati (2017) concernant les secteurs très médicalisés. Et contrairement à ce que soutient Mark Radcliffe (2000), cette perméabilité des frontières interprofessionnelles ne relève pas d'une "erreur fondamentale» consistant à "confondre égalité et uniformité» (p. 1085), mais bien d'un contexte de soins où "la rapidité des événements et la nécessaire rapidité des actions rendent pratiquement impossible aux infirmières et aux médecins de maintenir des juridictions clairement séparées" (Liberati, 2017, p. 140). Relevons toutefois que, comme le montre Anne Paillet (2007), cette double proximité physique et symbolique entre infirmières et médecins que l'on observe dans les secteurs très médicalisés ne suffit pas à effacer les cultures distinctes qui caractérisent les deux professions, notamment concernant les questions éthiques liées à la fin de vie des patients.

\section{Une autonomie spécifique}

Alors que la proximité avec le corps médical offre quelques avantages, elle comporte aussi un coût: celui d'une certaine limitation de l'autonomie professionnelle. Les dominantes médicales apparaissent en effet comme celles dont la pratique est le plus fortement marquée par les tâches médico-déléguées $(53,2$ vs $40,3 \%)$, et dont le sentiment d'autonomie est faible (17,9 vs 25,9\%). "Grandes infirmières» au sein du champ médical, elles courent ainsi toujours le risque d'apparaître comme de "petits médecins" dès lors qu'elles se trouvent resituées dans l'espace infirmier.

Il serait cependant erroné de ne voir dans ces réponses au questionnaire que la manifestation d'une absence d'autonomie. Rappelons d'abord que c'est parmi les secteurs les plus médicalisés que les tâches médico-déléguées sont les plus valorisées par les infirmières (Feroni \& Kober, 1995). Car si ces tâches 
constituent bel et bien un cadre relativement contraignant, il n'en reste pas moins que les infirmières peuvent les vivre comme des espaces à l'intérieur desquels elles jouissent d'une forme d'autonomie spécifique. Pour Madame Jacques, les tâches médico-déléguées contribuent clairement à l'attrait des soins intensifs en permettant aux infirmières qui y exercent d'être «autonomes sur délégation médicale», alors que les infirmières des secteurs hospitaliers moins médicalisés disposent selon elle de "moins de marge de manouvre»:

"Il y a des choses parfois où on est un peu autonomes sur délégation médicale. Ils [les médecins] nous donnent des cibles, un peu un cadre, et puis on manage certains médicaments dans ce cadre-là. [...] Dans tous les soins intensifs, ça se passe comme ça. À l'étage, je pense qu'il y a des choses où elles [les infirmières] ont moins de marge de mancuvre, je pense. [...] Je pense qu'on n'a pas la même... On a une formation différente et on gère pas les mêmes choses. Je veux dire de mon patient, je connais tout, hein, je veux dire, je connais ses pathologies, ses antécédents, je connais toute la fonction cardiaque, respiratoire, comment il urine, comment on l'alimente, je connais son laboratoire. Donc je peux manager les médicaments, parce que je sais. [...] Une infirmière en division, elle a pas le temps. Elle, elle a, je sais pas, dix patients ou peut-être même plus. Elle, elle ne peut pas savoir, elle n'a pas la même... euh... Elle ne peut pas décider de donner un médicament parce qu'elle ne connaît pas tout le reste. Nous, il y a des choses qu'on se dit: "On va mettre en route ça, mais on va arrêter ça." Et on en parle après à notre médecin. Et puis il dit: "Ben, t’as bien fait, ou pas bien fait."”

Comme nous le montrerons, cette valorisation du rôle médico-délégué ne se retrouve pas chez les infirmières situées au pôle infirmier de l'espace. Au contraire, et à l'instar de la position défendue par l'association britannique des infirmières, ces 
professionnelles perçoivent cette organisation du travail «comme un moyen de renforcement de la subordination traditionnelle des infirmières aux médecins» (FERONI \& Kober, 1995, p. 49). De fait, la délégation médicale non seulement suppose que les infirmières "doivent s'adapter au médecin, à sa pratique de travail, à ses habitudes, à sa disponibilité notamment pour la détermination du temps de la visite, espace-temps où le médecin effectue ses prescriptions", mais limite aussi tout développement d'un "rôle propre", "étant donné qu'un champ de compétence du travail infirmier n'est même pas défini" (Рісот, 2005, p. 87-88). En valorisant une telle organisation, les infirmières situées au pôle médical participent ainsi d'un certain conservatisme relatif au contenu de leur travail.

\section{Une distance avec les subalternes et les patients}

À la proximité relative que les dominantes médicales entretiennent avec les médecins fait écho la distance symbolique qu'elles établissent vis-à-vis des subalternes autant que des patients. Envers les premiers, une claire division du travail liée à des compétences spécifiques favorise la distinction entre les tâches qui leur reviennent et celles pour lesquelles elles se sentent surqualifiées et qui, partant, devraient être effectuées par les subalternes ${ }^{39}$. Concernant les patients, la proximité physique qu'impliquent les nombreux soins de confort et d'hygiène se voit compensée par une certaine mise à distance symbolique dont on sait qu'elle contribue au maintien de l'engagement professionnel (CASTRA, 2004) autant qu'à la préservation de l'identité professionnelle (VÉGA, 1997). Dotées d'un capital médical hautement légitime, les dominantes médicales tendent ainsi à reléguer les patients au rang de profanes dès lors qu'il

\footnotetext{
39 À la proposition "Je dois effectuer des tâches pour lesquelles je m'estime surqualifiée", les dominantes médicales sont $4,8 \%$ à répondre "toujours", contre 3,4\% de l'échantillon.
} 
s'agit d'évaluer leur état de santét ${ }^{40}$. La possession de ce savoir spécifique fait des secteurs les plus médicalisés des lieux dans lesquels la logique médicale impose sa légitimité, y compris face à des patients qui opposent des logiques hétérogènes. Évoquant le refus des Témoins de Jéhovah de subir des transfusions sanguines ou encore la réticence de certaines femmes de confession musulmane d'être prises en charge par des hommes, Monsieur Ropars écarte ces revendications en invoquant l'impératif de prise en charge médicalisée:

"S'il n'y a pas le choix, on explique et pis voilà; en général ils comprennent [...] ils comprennent que c'est un service de soins; que c'est comme ça et pis qu'ils sont soignés et pis que c'est comme ça [...]. "On voudrait bien pouvoir rentrer dans votre truc, mais on peut pas. Donc si vous ne voulez pas, on fera rien. Mais sinon, c'est ça." [...] En général, quand la vie est en jeu, on laisse les valeurs de côté."

Comme on le voit dans cet exemple, la dépendance des patients à l'égard du personnel hospitalier ("la vie est en jeu») contribue à l'imposition de la logique médicale. À la possession d'un fort capital médical s'ajoute donc un deuxième facteur de distance entre infirmières et patients, qui tient à la très forte médicalisation de ces derniers qui réduit fortement leurs possibilités d'intervention dans le travail infirmier. Que l'on pense seulement à la «situation standard» des soins intensifs telle que décrite par Terraneo, Seferdjeli et Diby (2010, p. 341), dans laquelle le patient est intubé, ventilé, sédaté, et est au minimum équipé d'un scope, d'un tube orotrachéal, d'un ventilateur, d'une sonde naso-gastrique, de cathéters veineux et artériel et d'une sonde vésicale. Dans de telles situations, la place pour des logiques autres que médicales est mince, voire nulle

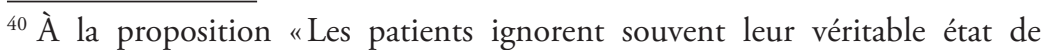
santé», les dominantes médicales sont $38,3 \%$ à choisir la modalité la plus favorable («tout à fait d'accord»), contre 34,4\% de l'échantillon.
} 
lorsque le comportement du patient est susceptible de péjorer son pronostic vital:

"Il y a des gens, ils se réveillent, mais ils sont franc fous, ils pourraient tout arracher! Et puis là, on n'a pas le temps. [...] Alors on leur dit vite: "Vous êtes aux soins intensiff, vous avez un tube dans la gorge", mais en même temps on les endort déjà parce que c'est trop de risques. "Mme Jacques, soins intensifs

Si la distance relative que les infirmières maintiennent à l'égard des patients s'explique par leur possession d'un fort capital médical et de la situation de grande vulnérabilité des patients, elle s'explique aussi par la standardisation des prises en charge. Les dominantes médicales sont ainsi celles qui adhèrent le moins à la proposition "chaque infirmière a la possibilité de développer sa propre manière de donner les soins" (9,7 vs $14,8 \%)$. Tout se passe comme si l'insensibilité caractéristique de la médecine aux variations contextuelles (et notamment nationales) mise en évidence par Vassy (1999) avait tendance à se diffuser à la profession infirmière au pôle médical, qui présente une standardisation que l'on ne retrouve pas - ou que dans une bien moindre mesure - au pôle infirmier. La manière dont Monsieur Bideau, qui exerce en anesthésie, use du terme de "cas" pour désigner la prise en charge des patients est à cet égard révélatrice:

"J'aime bien justement quand on peut commencer un cas et pis finir un cas. [...] Nous, en anesthésie, on a encore la chance d'avoir un travail où on commence et pis on finit, pis ben ce qui était avant... ben, c'était avant et pis après, ben, ça nous concerne plus vraiment, c'est... On a passé le relais et pis c'est terminé. Alors j'aime pas quand... quand on peut pas finir un cas."

On le voit, le "cas» est ici entendu comme une prise en charge étroitement protocolée dont les bornes de début et de 
fin sont définies selon des critères exclusivement médicaux. Étant toujours le produit d'une sélection médicale en amont et appelé à une nouvelle prise en charge en aval, il suppose une très forte division du travail, et donc une spécialisation dont on sait qu'elle dispense largement les professionnels de la prise en compte d'éléments étrangers à la logique médicale (FrEIDSON, 1963). La notion de "cas» s'oppose en cela frontalement à celle de "personne", si souvent employée par les infirmières situées au pôle infirmier, et qui renvoie à une prise en charge "globale» excluant d'emblée l'exclusivité de la logique médicale.

\section{Encadré 1 : Sandrine Jacques, une «dominante médicale » ${ }^{41}$}

Madame Jacques a 41 ans. Fille d'une assistante dentaire de formation et d'un père maître d'apprentissage, elle a grandi dans une ville importante de la région où elle vit aujourd'hui en couple avec un fonctionnaire de police. Elle a une petite sœur qui exerce comme secrétaire. Sa maturité fédérale en poche à la fin des années 1980, elle s'oriente directement vers les soins; un choix du «milieu de la santé » qu'elle présente comme assez évident:

"-En fait, je crois que j'ai toujours su que je voulais faire quelque chose dans le milieu de la santé, mais après je ne sais pas exactement quel métier j'ai voulu faire [...] Je savais pas exactement quoi. Je pensais physiothérapeute, je pensais peut-être infirmière... Enfin, j'avais pas vraiment de trucs très définis.

- Vous savez d'où venait cette envie?

- Depuis toute petite, je crois, parce qu'on m'a toujours dit que j'aimais bien prendre soin des autres et pis que je m'occupais des autres; et déjà à l'école, je m'occupais des plus petits et tout ça quand même. Mais c'est quelque chose qu'on m'a raconté, je m'en souviens pas vraiment en fait.»

\section{"Là je me dis que je veux être physio"}

Si Madame Jacques présente l'envie de "prendre soin des autres» comme une sorte de prédisposition allant de soi, on peut également

${ }^{41}$ Ce portrait est issu de Toffel (2018). 
rattacher ce choix à l'influence d'un oncle perfusionniste du côté paternel: «J'ai toujours aussi été un peu imprégnée de ce qu'il pouvait nous raconter.»

Son premier contact avec le monde des soins sera un stage effectué en tant qu'aide-soignante au CHUV - en service de rhumatologie expérience qui confirmera son attirance pour la physiothérapie:

"Je fais un stage; et pis là je me dis que c'est vraiment... J'aime bien être au contact des gens, des patients, tout ça, c'est pas un milieu qui m'est pénible d'être à l'hôpital; pis là je me dis que je veux être physio.»

Après ce stage, elle fait donc le choix de s'orienter vers la physiothérapie, une ambition que l'on peut saisir à l'aune de deux modalités: son habitus de classe qui, alors même que son diplôme gymnasial lui aurait ouvert les portes de l'université ${ }^{42}$ - et de la faculté de médecine par exemple -, l'oriente vers une formation non académique, et le choix d'une profession de santé très technique lié au contact de son oncle. Cette affinité pour les soins techniques se retrouvera dans la suite de son évolution professionnelle.

\section{Des dimensions somatique et corporelle plutôt que "relationnelles»}

Suite à un échec aux examens d'entrée en physiothérapie, Madame Jacques prolonge son stage en rhumatologie. Elle opte alors pour une formation en soins infirmiers qu'elle entreprend à la rentrée scolaire suivante. Sorte de nécessité faite vertu, ce choix lui apparaît comme le résultat d'une meilleure connaissance du métier acquise au travers du stage en rhumatologie:

"Je me suis orientée plutôt vers infirmière parce que du coup j'ai aussi découvert ce métier, ce qu'on faisait; et puis je me suis dit que c'était plus... ça me semblait plus varié que physiothérapeute.»

L'intérêt pour les dimensions somatique et corporelle des soins semble être une récurrence dans les choix qu'elle opère dans sa carrière, et ce dès les stages effectués durant la formation. Ainsi, les stages qu'elle a le moins aimés sont ceux de la psychiatrie ou

\footnotetext{
${ }^{42}$ Rappelons que Madame Jacques débute la formation en soins infirmiers à la fin des années 1980, soit plus de 10 ans avant que celle-ci devienne académique avec l'introduction de la filière de formation bachelor en Suisse romande (2002) et près de 20 ans avant l'ouverture d'une filière master à l'université (2009).
} 
de l'EMS; ce dernier service qu'elle oppose directement aux soins aigus:

"Je savais que je voulais rester dans le milieu vraiment hospitalier et pis plutôt soins, de soins aigus, pas aller ni en EMS ni en convalescence, en tout cas pour les premières années de travail.»

Madame Jacques est diplômée en soins généraux en 1994. Si le choix de l'hôpital et "des soins aigus» est cohérent avec les affinités qu'elle entretient avec les soins techniques, le rejet qu'elle manifeste envers l'EMS ou « la convalescence» rappelle une opposition qui traverse l'ensemble de l'espace infirmier et qui semble présente dès le départ de la formation. La présentation de ce choix est typique de ces "goûts [qui] sont sans doute avant tout des dégoûts» (Bourdieu, 1979a, p. 60). II s'avère en effet que la structure de l'espace infirmier différencié et hiérarchisé est présente dès la formation en soins, notamment par le biais des bruits de couloirs colportés par les étudiantes des années supérieures ${ }^{43}$. Revenant des stages, celles-ci contribuent à façonner chez les nouvelles entrantes des représentations véhiculées dans l'ensemble de la profession comme une division entre des soins aigus hautement valorisés et valorisants, et des soins relationnels relativement peu intéressants.

\section{Toujours plus de soins aigus}

Madame Jacques débute sa carrière en hôpital orthopédique. Elle s'oriente là, dit-elle, "parce qu'il y avait une place, un poste et puis je me disais: "Voilà tu commences comme jeune infirmière" [...] tout était bon à prendre en fait hein.» $\mathrm{Si}$ «tout est bon à prendre», on comprend implicitement dans ses propos que le champ des possibles envisagés doit rester inscrit dans le milieu hospitalier. Bien qu'elle commence par travailler trois ans en orthopédie, son mouvement vers les soins aigus est entamé au bout d'une année seulement. Travaillant d'abord «en division» (dans les chambres), Madame Jacques poursuit avec deux années en salle de réveil et aux soins continus: "parce que déjà, j'avais l'envie d'aller faire quelque chose d'un peu plus vraiment aigu.» La trajectoire de Madame Jacques est donc très clairement

\footnotetext{
${ }^{43}$ Et probablement aussi par le truchement du discours d'enseignants ayant incorporé cette hiérarchisation de l'espace professionnel alors qu'ils exerçaient eux-mêmes au chevet du patient.
} 
orientée vers le technique et l'aigu, notamment dans une optique de complexification des prises en charge:

"Ça suit un degré de complexité en fait si vous voulez; parce que par exemple de passer de division dans des chambres aux soins intensifs, ça fait un gros... un gros saut en fait. »

Parce que Madame Jacques envisage assez rapidement une évolution professionnelle, elle entame ensuite une formation post-diplôme "pour un peu évoluer» et s'oriente alors vers les soins intensifs; service dans lequel elle exerce toujours.

On peut saisir le désir d'«évoluer» de Madame Jacques tant par ses dispositions que par un état de la structure de l'espace infirmier. Bien que la profession offre une large panoplie de secteurs d'activité, les possibilités de progressions professionnelles y sont en fait relativement limitées. Outre les fonctions de cadres et de cliniciennes spécialisées qui éloignent tant soit peu des patients, et les (récentes) formations universitaires qui nécessitent un appui institutionnel et des conditions familiales qui rendent possible d'entreprendre un retour aux études ${ }^{44}$, la progression passe souvent, dans les secteurs médicalisés, par l'acquisition et l'accroissement du capital médical. Or, seuls les services les plus médicalisés (urgences, soins intensifs, anesthésie, soins continus) offrent des formations «certifiantes». Aussi, lorsque Madame Jacques ressent "l'envie d'aller un peu plus loin», c'est tout logiquement - en raison d'une structure et d'une offre de formation de l'espace infirmier d'alors - qu'elle s'oriente vers les soins intensifs:

«C'est vrai qu'on en parlait et on était trois [en orthopédie] à avoir la même envie d'aller un peu... un peu plus loin aussi et pis ben de continuer à faire une formation, pis de gratter un peu ce côté soins techniques, et pis à apprendre un peu plus au niveau des pathologies, et tout ça.»

Si le choix d'un service aigu et très technique est, on le voit, tout à fait cohérent avec une trajectoire débutée très tôt, Madame Jacques ne manque pourtant pas de relever la dimension relationnelle des soins prodigués dans ce service:

"Ben, je m'étais dit: "Ouais les soins intensifs, ça devrait bien me plaire, ça devrait aller; il y a un côté technique et pis un côté quand même avec un lien avec le patient". »

\footnotetext{
${ }^{44}$ Formations conduisant à exercer en tant que cliniciennes spécialisées, postes qui éloignent également de la pratique clinique.
} 
Alors que la trajectoire de Madame Jacques semble guidée par l'acquisition de compétences techniques, il est intéressant de relever la façon dont elle s'empresse de mettre en avant la dimension relationnelle de son activité. On peut donc considérer que le «technique pur» fait figure d'image repoussoir de la profession.

"On aime ça quand même, sinon on viendrait pas aux soins intensifs» $\mathrm{Si}$, comme on l'a dit, le prestige dont les services aigus sont auréolés est conféré par la technicité et la proximité avec les médecins, il faut ajouter à cela le fait qu'au sein de ces services, les infirmières ne s'occupent que d'un ou deux patients, dont elles ont la complète responsabilité. Une responsabilité d'autant plus grande que seuls les cas graves - pronostic vital souvent engagé - sont pris en charge dans ces services:

«Parce que ça a un côté quand même assez... ben il y a des choses qui sont stressantes, mais en même temps, y a des trucs ben voilà... quand ça va pas, on a de l'adrénaline pis on aime quand même ces situations un peu d'urgence où il faut aller vite, il faut... il faut pas se louper... il faut repérer les trucs, il faut anticiper, tout ça. On aime ça quand même, sinon on viendrait pas aux soins intensifs; et puis après quand les gens s'en sortent et pis que ça va bien, et pis que... voilà, qu'on a gagné, on est quand même content. »

Ici, la dimension vitale trouve un prestige tout particulier. Le fait qu'il ne faut "pas se louper», et que "les gens s'en sortent», "qu'on a gagné», est solidaire de la perspective de "guérison» qui a cours dans ces services; ceci à l'opposé d'une conception de l'accompagnement du patient qui prévaut en particulier dans les secteurs situés au pôle infirmier de l'espace:

"Alors très souvent c'est des gens qui sont restés assez longtemps qui reviennent, avec qui on a eu le temps de nouer plus une grande relation et tout ça. Parce que quand on voit des gens qui... qui ont eu des gros accidents de voiture ou de moto et pis qui sont partis qui étaient encore un peu comateux comme ça, pis qui viennent vous revoir sur leurs deux jambes, pis qui vous disent qu'ils se sont mariés, tout ça, c'est super chouette parce qu'on se dit: "Ben, ça valait la peine de se battre."» 
Cette satisfaction d'avoir pu agir, et d'en voir les résultats, est une source directe de satisfaction dans ces secteurs où la vie des patients est souvent en jeu.

\section{"On est un peu autonomes sur délégation médicale»}

L'une des caractéristiques des dominantes médicales - et qui les rend particulièrement emblématiques d'une vision que d'aucuns, notamment dans la formation, considèrent comme surannée repose sur l'acceptation d'une position subalterne aux médecins; ce que nous avons présenté avec les réponses portant sur le qualificatif de l'infirmière comme «bras droit du médecin». Madame Jacques adhère à cette conception, bien qu'elle y ajoute une nuance de taille, lorsqu'elle affirme que les infirmières qui exercent aux soins intensifs sont "un peu autonomes sur délégation médicale». Cette expression n'est contradictoire qu'en apparence. En fait, tout se passe comme si le travail délégué était une dimension de son travail qu'elle avait complètement intégrée (il n'existe pas d'alternative, pas de "rôle propre»). Dès lors, ce qui distingue les infirmières n'est pas tant la dimension plus ou moins autonome (au sens de rôle propre), mais la plus ou moins forte marge de manœuvre à l'intérieur d'un rôle médico-délégué délimité. Cette aisance se manifeste au travers du rapport que Madame Jacques entretient avec les aide-soignantes. Alors qu'on peut observer une certaine indifférenciation des rôles entre les aide-soignantes - a fortiori les assistantes en soins et santé communautaire (ASSC) - et les infirmières dans les services les moins techniques, Madame Jacques mentionne une claire division du travail dans son service:

"Les aide-soignantes, elles nous aident dans les soins de base, c'està-dire les toilettes [...] Et puis chez les patients intubés, parfois, par exemple elles commencent à faire la toilette, pis nous on est là en train de donner des médicaments; donc on est avec elles et puis... voilà après on les aide aussi pour tourner, pour refaire le lit tout. Sinon, après elles ont aussi les tâches un peu ménagères, on va dire. Nettoyage du box, remplissage de matériel et tout ça; et puis aussi discuter avec les gens avec certains... justement quand elles ont leur ménage là, ben avec les patients avec qui elles peuvent parler, ben... voilà c'est aussi un côté relationnel aussi... important.»

Plus les services sont aigus, plus la division des tâches, reposant sur des compétences et des connaissances spécifiques 
- physiopathologiques ou pharmacologiques -, est clairement visible. D'un côté, il y a les soins d'hygiène et de confort qui reviennent aux aide-soignantes tandis que de l'autre, les infirmières sont «là en train de donner des médicaments». Par ailleurs, et bien que Madame Jacques revendique - on l'a vu - un aspect relationnel des soins même dans cette région des plus médicalisée de l'espace, on remarque qu'elle attribue clairement aux aide-soignantes le rôle de «discuter avec les gens», autrement dit: le «côté relationnel».

Par une trajectoire ascendante marquée par les soins hospitaliers, et en particulier dans les soins aigus, la dominante médicale apparaît avant tout comme celle qui, exerçant dans les régions les plus prestigieuses de l'espace infirmier, incarne la «technicienne» par excellence. Proche des médecins et ne remettant pas frontalement en cause une hiérarchie hospitalière, ses prises de position sont finalement très conservatrices concernant les rapports qu'entretient la profession à ses collègues médecins. 



\section{Chapitre 6}

\section{Les dominées médicales}

La fraction située en bas à droite de l'espace est celle qui rassemble le plus d'individus (graphique 5). Ces infirmières exercent prioritairement en pédiatrie (12,7 vs $9,3 \%)$, chirurgie (11,4 vs $8,3 \%)$, rééducation $(5,4$ vs $4 \%$ ) et médecine ( 4,4 vs $3,2 \%$ ), soit des secteurs qui occupent une position intermédiaire sur l'échelle de prestige de la profession.

Le faible volume de capital de ces infirmières se manifeste en de multiples indices, que ce soit la mobilisation relativement faible des diverses compétences et savoirs (infirmiers comme médicaux), l'absence relative de formations post-diplômes (32,3\% ne disposent que d'un diplôme de base, contre 18,6\% de l'échantillon) ou la limitation à des formations courtes (29 vs $18,8 \%$ ), ou encore un taux d'activité peu élevé (seules 34,6\% exercent à plus de $80 \%$, contre 44,5\% de l'échantillon). Ce faible volume est associé à une structure à dominante médicale, comme en atteste le fait que ces infirmières suivent des formations à orientation moyennement médicale (30,5 vs 18,6\%), mobilisent plutôt leurs connaissances en physiopathologie $(63,2 \%$ répondent "souvent» contre 46,8\% de l'échantillon) et en machine (48,1\% de «souvent» contre $28,6 \%$ de l'échantillon) et ne s'appuient que rarement sur leurs compétences relationnelles $(55,5$ vs $74,2 \%)$, 
leur intuition (11,9 vs $29,5 \%)$ ou encore leurs connaissances en modèles infirmiers (3,3 vs $17,8 \%)$.

À la différence des trois autres fractions qui, nous le verrons, luttent pour une définition spécifique des pratiques légitimes, on ne repère pas de véritable "projet émancipateur" chez ces infirmières. Ainsi, et bien qu'elles soient les plus nombreuses, elles ne peuvent être décrites qu'en "creux» dès lors qu'elles sont comparées aux trois autres fractions, et notamment aux dominantes médicales dont elles présentent la même structure de capital avec toutefois un volume moins élevé. Raison pour laquelle nous les qualifions de "dominées médicales» ${ }^{45}$.

\section{Une « misère de position »}

Les secteurs dans lesquels exercent les dominées médicales occupent une position intermédiaire sur l'échelle de prestige, mais c'est pourtant ici - et non parmi les secteurs situés au plus bas de cette échelle - que cette position dominée est ressentie de la manière la plus forte. Exerçant en médecine, Monsieur Pichoud déclare ainsi que "de façon assez générale, les gens qui travaillent aux soins intensifs, anesthésie, ils ont un statut plus élevé que nous». Cette reconnaissance participe d'un processus de domination symbolique qui intervient toutes les fois que les pensées et perceptions des individus «sont structurées

\footnotetext{
45 Contre toute dérive essentialiste, il convient de préciser que la description «en creux» que nous proposons ici est à considérer en regard de notre échelle d'analyse d'une part (nous nous situons à l'échelle de l'espace infirmier dans son ensemble, et non à celle de secteurs particuliers), de la théorie des champs que nous mobilisons d'autre part, théorie dont on sait qu'elle privilégie une posture légitimiste plutôt que relativiste (Grignon \& Passeron, 1989). Parce qu'ils privilégient une autre échelle d'analyse et reposent souvent sur d'autres cadres théoriques, de nombreux travaux monographiques permettent de saisir toute la richesse et la complexité de l'activité des infirmières appartenant à la fraction des "dominées médicales» (Acker, 2005; Lanza, Longchamp, SEFERdJEli \& Müller, 2004; VÉGA, 1997, 2000).
} 
conformément aux structures mêmes de la relation de domination qui leur est imposée» (Bourdieu, 1998, p. 27). Lorsqu'il lui est demandé comment est perçu son secteur d'activité dans la profession, Madame Dunod, qui travaille également en médecine, établit immédiatement la comparaison avec les infirmières des soins intensifs:

"Par exemple, si on regarde la relation qu'on a avec les soins intensifs, ils pensent qu'on fait rien du tout, que eux c'est tellement technique que ce qu'on fait c'est vraiment... [...] On le sent... Quand on va chercher des patients qui peuvent venir en médecine, [...] c'est vrai qu'ils nous causent comme si on comprenait pas ce qu'étaient les médicaments ou comme ça. Alors qu'on les utilise aussi souvent qu'eux. Peut-être aux urgences c'est différent, je sais pas. Mais soins intensifs, c'est un milieu très fermé quoi [...] Pour eux, notre service, c'est comme si c'était se rabaisser à venir nous aider à faire des toilettes quoi.»

À la différence des infirmières qui exercent dans les secteurs situés au plus bas de l'échelle de prestige, les dominées médicales se retrouvent majoritairement dans des secteurs hospitaliers et sont de ce fait au contact direct des infirmières exerçant dans les secteurs les plus prestigieux. Elles vivent ainsi ce que Pierre Bourdieu (1993) qualifie de misère de position, soit une misère relative qui peut affecter ceux qui, tel le contrebassiste au sein d'un orchestre symphonique, occupent une position inférieure au sein d'un univers prestigieux «auquel ils participent juste assez pour éprouver leur abaissement relatif " (p. 11).

À cette misère de position s'ajoute pour les dominées médicales un faible sentiment d'employabilité (8,1 vs 12,9\%) - qui les distingue notamment des dominantes médicales - ainsi qu'un revenu plus faible que parmi les autres fractions ${ }^{46}$. Par

${ }^{46}$ Le revenu équivalent plein temps d'une femme de 43 ans (âge moyen de notre échantillon) appartenant à cette fraction s'élève à 6167 francs, soit 
ailleurs, et bien que les tâches médico-déléguées soient moins omniprésentes que chez ces dernières, les dominées médicales présentent le sentiment d'autonomie le plus faible de toutes les fractions $(17,4$ vs 25,9$)$. Un sentiment qui se manifeste aussi dans la conception qu'elles se font de la profession, puisqu'elles sont celles qui adhèrent le moins à l'idée suivant laquelle les soins infirmiers devraient développer un savoir propre (13,9 vs 23,6).

\section{Une position d'intermédiaire entre patients et médecins}

Nous avons vu dans le chapitre précédent que la relation qu'entretiennent les dominantes médicales avec les médecins se caractérise par une relative indifférenciation des tâches. Une telle relation ne se retrouve pas parmi les dominées médicales qui semblent maintenues à plus grande distance par les médecins. Plus précisément, ces infirmières occupent une position d'intermédiaire entre patients et médecins. C'est en effet au sein de cette fraction que l'on trouve la tendance la plus forte des infirmières à jouer le rôle de "répétitrices et traductrices des informations médicales" (ACKER, 2005, p. 167). Exerçant en médecine, Monsieur Pichoud décrit cette fonction de l'infirmière:

"Nous on parle des problèmes qu'on a constatés [...] ou que le patient nous a décrits. Et puis, il [le médecin] regarde le laboratoire, donc les prises de sang qui ont été faites [...] et pis on complète s'il $y$ a des choses qui sont pas dites par le patient. Parce que souvent les patients avec nous, ils ont beaucoup de demandes... Enfin, ils disent: "Il faut que je

234 francs de moins que la moyenne de l'échantillon. Pour les revenus moyens des différentes fractions non contrôlés par d'autres variables, voir tableau A4 en annexe 3. 
dise au médecin ça, ça, ça"; alors nous on prend des notes; et puis [...] quand ils voient le médecin, des fois il y a soit la peur du médecin ou [...] ils savent qu'ils ont peu de temps, donc ils oublient leur question. Alors on dit: "Ah ils nous ont parlé de ça ce matin" ou souvent moi je dis aux gens: "Écrivez une petite liste, vos problèmes, comme ça, vous y pensez à la visite médicale."”

La spécificité du rôle infirmier repose notamment ici sur une présence continue auprès des patients d'une part, et une disponibilité vis-à-vis des médecins d'autre part. La relation entre infirmières et médecins se rapporte donc à un "modèle de séparation» (Liberati, 2017, p. 138) qui se distingue de la relative indifférenciation des rôles qui a cours dans les secteurs les plus médicalisés en raison de la célérité que nécessitent les prises en charge.

Cette position d'intermédiaire entre patients et médecins peut laisser penser que les dominées médicales constituent une sorte d'archétype de l'infirmière attachée à une "santé globale» tenant compte des désirs des patients et des familles. Mais cette vision largement congruente avec la représentation commune du rôle infirmier ne résiste pas à l'analyse sociologique menée ici: interrogées sur le degré auquel les soins qu'elles prodiguent intègrent les désirs des patients et de leurs familles, les dominées médicales ne se distinguent pas du reste de l'échantillon, et y adhèrent significativement moins que les élites infirmières et les hétérodoxes (voir chapitres 7 et 8$)^{47}$.

Au final, les dominées médicales apparaissent comme les plus dominées de l'espace infirmier. Si leur position leur assure une

\footnotetext{
${ }^{47}$ Les deux propositions étaient: "dans mon secteur d'activité, les soins intègrent les désirs des patients" et "dans mon secteur d'activité, les soins intègrent les désirs des proches/familles des patients ». Les dominées médicales sont respectivement 84,1 et $73,5 \%$ à répondre "s'applique partiellement " ou «s'applique parfaitement», soit un taux équivalent à celui de l'échantillon total.
} 
certaine proximité physique avec les médecins, la faiblesse de leur volume de capital empêche la mise à distance des subalternes et des patients, ce qui entraîne une moindre assise identitaire. $\mathrm{Ne}$ demeure alors que l'absence d'autonomie, que ces infirmières ne peuvent contrebalancer en raison de leur relative dépossession de capital infirmier. 


\section{Chapitre 7}

\section{Les élites infirmières}

Situées en haut à gauche de l'espace (graphique 5), les infirmières de la troisième fraction se distinguent par des formations postgrades de niveau long $(74,3$ vs 55,9$)$, voire académique $(11,8$ vs 6,7$)$, ainsi que par un taux d'activité élevé $(58,8 \%$ d'entre elles exercent à plus de $80 \%$, contre $44,5 \%$ de l'échantillon). Ces infirmières exercent dans les secteurs de l'enseignement et de la recherche $(10,5$ vs 4,0$)$ et de la psychiatrie $(10,5$ vs 7,8$)$, ou occupent des fonctions de cadre $(37,4$ vs 17,3$)$. La forte mobilisation des compétences relationnelles $(94,4$ vs 74,2$)$, des valeurs personnelles $(71,3$ vs $36,4)$ et de l'intuition $(52,1$ vs 29,5$)$, l'assistance à des congrès de soins infirmiers $(59,5$ vs 41,0$)$ et l'affiliation à l'ASI $(42,0$ vs 33,3 ) attestent d'une structure de capital à dominante infirmière, avec une très forte composante scientifique manifestée par la mobilisation de connaissances en recherche $(39,4$ vs 14,0$)$ et en modèles théoriques infirmiers (43,0 vs 17,8$)$, ou encore par des formations orientées vers la recherche et l'enseignement $(21,7$ vs 11,7$)$. Ces infirmières présentent donc un volume de capital élevé, ce qui les rapproche des dominantes médicales. Mais à la différence de ces dernières, elles se caractérisent par une structure de capital à dominante infirmière qui les situe au pôle opposé de l'espace infirmier. Participant activement à 
la lutte pour la constitution d'un savoir scientifique spécifique et disposant d'un accès privilégié à la formation initiale des futures professionnelles, elles exercent une influence décisive sur l'ensemble de la profession. Dans la continuité des travaux d'André Petitat (1989) qui parle d'«élite» pour désigner les cliniciennes au Canada (p. 74), nous qualifierons donc ces infirmières $\mathrm{d}^{\prime}$ «élites infirmières ${ }^{48}$.

\section{En lutte pour une autodétermination professionnelle}

Il convient de relever que la possession d'un fort capital infirmier n'est pas ici associée à des pratiques hétérodoxes: les élites infirmières ne se caractérisent pas par des formations paramédicales ou de développement personnel $(1,6$ vs 9,2) et, surtout, elles affichent la même réticence que leurs collègues du pôle médical face aux congrès de médecine complémentaire ${ }^{49}$. Cette absence d'hétérodoxie n'est certainement pas étrangère à la présence d'une forte composante managériale qu'atteste la surreprésentation des formations en management et encadrement parmi cette fraction $(35,6$ vs 16,5$)$. Mais cette caractéristique des élites infirmières ne doit pas non plus être confondue avec

\footnotetext{
${ }^{48}$ C'est parce que l'influence qu'exercent ces infirmières sur la profession est plus déterminante que celle exercée par les dominantes médicales que nous les qualifions d' "élites infirmières", et non de "dominantes infirmières". Alors que la notion d'élite est généralement mobilisée pour désigner «les groupes sociaux qui, en raison de leur position ou de leurs ressources, sont en mesure d'influencer l'évolution de la société en participant aux décisions importantes de celle-ci» (Hartmann, 2007: 17), elle nous semble particulièrement adaptée pour qualifier la fraction d'infirmières dont il est question ici.

${ }^{49}$ Une écrasante majorité d'entre elles $(96,4 \%)$ n'a assisté à aucun congrès de médecine complémentaire durant l'année précédant l'enquête, soit une proportion semblable à celles observées parmi les dominantes médicales et les dominées médicales (respectivement 99,7 et 97,4\%), mais sensiblement plus élevée que celle observée parmi les hétérodoxes (84,0\%).
} 
l'orthodoxie médicale observée chez les dominantes médicales. Car ces infirmières participent activement à la lutte pour une autonomisation spécifique de la profession ${ }^{50}$.

Ainsi, les élites infirmières adhèrent plus que les autres fractions à la proposition suivant laquelle la profession infirmière devrait développer un savoir qui lui soit propre $(35,9$ vs 23,6$)$, tout en rejetant l'image traditionnelle de l'infirmière comme «bras droit» du médecin ${ }^{51}$. Sans doute favorisées par la faible médicalisation de la région de l'espace qu'elles occupent, ces prises de position correspondent néanmoins à des dispositions profondes, comme en témoigne le fort sentiment d'autonomie de ces infirmières (35,3 vs 25,9$)$, qui les distingue encore des fractions du pôle médical.

Symbole de leur autonomie, le «savoir propre» que tentent de développer les élites infirmières est aussi brandi comme arme permettant de dépasser l'insatisfaction et la souffrance exprimées par certaines collègues. Ce qu'illustrent les propos de Madame Fabre, infirmière clinicienne spécialisée en médecine:

"Le côté très plaintif qu'ont parfois ou souvent les infirmières à se lamenter sur leur sort au lieu de pouvoir faire quelque chose; on perd du temps à se plaindre, mais on pourrait très bien faire quelque chose de bénéfique au lieu de se plaindre dans le même temps. Moi je pense qu'il faut être très bien formé et pis même si on a peu de temps, ben, si on a des compétences développées ben on peut les mettre en application auprès des patients, et pis avoir des résultats."

\footnotetext{
${ }^{50}$ Cette autonomisation passe entre autres par le fait de minimiser la hiérarchie avec les médecins. Les élites infirmières voient ainsi d'un bon œil la disparition des anciens médecins-chefs qui étaient "là tout le temps" et leur remplacement par de nouveaux profils médicaux face auxquels elles pensent pouvoir accroître leur autonomie.

${ }^{51}$ Elles sont $42,3 \%$ à s'opposer à cette proposition (contre 35,9\% de l'échantillon).
} 
En présentant la formation comme un moyen de faire face à l'accélération des séjours hospitaliers, Madame Fabre se distingue de la posture que l'on observe habituellement au sein de la profession, et qui consiste à considérer cette accélération comme un obstacle au rôle d'écoute et d'accompagnement des patients (ACKER, 2005). Ainsi, et à la différence des infirmières hétérodoxes qui, nous le verrons, présentent de fortes dispositions subversives, les élites scientifiques apparaissent davantage comme des agents d'accompagnement des réformes managériales en cours dans le système de santé ${ }^{52}$.

Un capital infirmier à composante scientifique et managériale éloigne les élites infirmières de la pratique clinique $(35,2 \%$ d'entre elles n'ont jamais affaire à des patients, contre 13,6\% de l'échantillon) et donc de la confrontation directe avec les médecins. Reposant pour partie sur un savoir propre à la profession, ce capital assure à ces infirmières une certaine assise identitaire, ainsi qu'un revenu élevé ${ }^{3}$. Infirmière clinicienne spécialisée en médecine, Madame Genoud relève ainsi que, contrairement à celles qu'elle qualifie d'"infirmière[s] de terrain " ${ }^{54}$, celles qui ont suivi la même formation qu'elle (un

\footnotetext{
${ }^{52}$ Il existe certes un courant critique au sein des sciences infirmières, incarné par les «savoirs émancipatoires" (Chinn \& Kramer, 2008). Ce courant demeure cependant marginal dans la discipline. Aant Elzinga (1990) relève le fait que les sciences infirmières, fortement influencées par la perspective théorique systémique, demeurent largement insensibles à l'analyse des relations de pouvoir et s'accommodent aisément du processus de «rationalisation» des soins. Dans la même veine, Annette J. Browne (2001) montre que la plupart des théories de sciences infirmières, ignorant les déterminismes de race, de classe et de genre, sont sous-tendues par l'idéologie libérale d'un individu rationnel et libre de ses choix.

${ }^{53}$ Le revenu équivalent plein temps d'une femme de 43 ans (âge moyen de notre échantillon) appartenant à cette fraction s'élève à 6787 francs, soit 386 francs de plus que la moyenne de l'échantillon. Pour les revenus moyens des différentes fractions non contrôlés par d'autres variables, voir tableau A4 en annexe 3.

${ }^{54}$ Dans les discours des infirmières titulaires du master, l'expression «infirmières de terrain» désigne les infirmières qui, non titulaires de ce
} 
master en sciences infirmières) ont une capacité à "être à l'aise avec leur discipline».

Les élites infirmières participent à la lutte pour une définition spécifique de la profession. En cherchant à rompre avec l'identité classique des infirmières exclusivement définie par la relation directe au patient, elles tentent de réhabiliter le «travail d'organisation » qui, bien que formant une part très importante de l'activité infirmière, est le plus souvent considéré comme le «sale boulot»(Allen, 2014).

\section{Les infirmières titulaires d'un master: entre émancipation et tensions professionnelles ${ }^{55}$}

S'inscrivant dans un mouvement d'académisation généralisé de la formation en soins en Europe (Lahtinen, Leino-Kilpi \& Salminen, 2014), l'Université de Lausanne a ouvert en 2009 une filière master en sciences infirmières à l'Institut universitaire de formation et de recherche en soins (IUFRS). Parmi les infirmières qui ont répondu à notre questionnaire, 44 sont titulaires d'un tel master ${ }^{56}$. Bien que peu nombreuses en raison de l'introduction récente de cette formation, elles symbolisent l'académisation et un certain avenir de la profession. On sait en effet l'importance de la scientifisation ${ }^{57}$ des savoirs pour l'autonomisation des aires de savoirs professionnels.

master, prodiguent directement des soins aux patients. Elles sont distinguées des infirmières «cadres» qui ne s'occupent plus directement des patients.

${ }^{55}$ Les résultats présentés dans cette section ont été publiés dans ToffeL (2020).

${ }^{56}$ Il s'agit là d'un chiffre élevé, compte tenu du fait que l'Université de Lausanne n'avait délivré que 60 diplômes de master au moment de l'enquête. ${ }^{57} \mathrm{La}$ scientifisation est entendue comme le tournant qui permet la légitimation d'une profession par la reconnaissance des savoirs et techniques qui la fondent. Voir Аввотт (1988: 195 et suiv.). 
Seules trois des quarante-quatre titulaires du master de l'IUFRS se situent au pôle médical de l'espace (deux parmi les dominantes médicales, et une parmi les dominées médicales). Parmi les quarante et une restantes, douze se situent parmi les hétérodoxes, et vingt-neuf - soit une écrasante majorité - parmi les élites infirmières. On peut ainsi considérer que ces infirmières forment une sous-catégorie des élites infirmières.

Les infirmières titulaires $\mathrm{du}$ master sont sensiblement plus jeunes que la moyenne de l'échantillon (38,5 vs 43 ans) et présentent une proportion d'hommes plus élevée (20 vs 15,5\%). Elles ont plus souvent un père au bénéfice d'un diplôme du secondaire professionnel supérieur (37,2 vs $27,9 \%)$ ou d'une formation universitaire (21,2 vs $13,0 \%)$, ce qui semble faire écho aux considérations d'Aant Elzinga (1990) suivant lesquelles l'académisation de la formation en soins est susceptible de faire advenir une «petite bourgeoisie» au sein de la profession infirmière.

Comme en témoigne le fait qu'elles sont deux fois plus enclines que leurs collègues $(81,8$ vs $41,8 \%)$ à considérer que la profession devrait développer des savoirs scientifiques propres, ces infirmières entendent incarner un renouvellement de la profession. Pour ce faire, elles occupent souvent des postes d'enseignement et de recherche au sein des Hautes écoles spécialisées (29,5\%), et plus souvent encore le statut d'infirmières cliniciennes spécialisées (ICLS) dans des hôpitaux universitaires (50,0\%). Si l'on peut considérer, avec Dubet (2002), que les infirmières "ont le sentiment de toujours courir entre plusieurs définitions de leur métier, et dont aucune ne les satisfait pleinement» (p. 195), il semble que les infirmières titulaires du master soient en passe de mettre un terme à cette course effrénée en proposant une définition spécifique du métier qui repose sur une expertise scientifique des soins. C'est ainsi que Madame Demaya, enseignante dans une HES, voit dans la formation master une manière de "valoriser» la profession:

"Quand ma collègue elle m'a dit: "ben [le master], c'est l'opportunité finalement de valoriser notre profession, pis de 
la mettre en avant. Et de montrer finalement qu'on peut aussi être experte des situations qu'on rencontre", je pense que c'est ça aussi qui m'a motivée."

Formées afin d'"optimiser la qualité des soins, la sécurité des patientes et patients" et de "collaborer aux changements organisationnels et aux évolutions nécessaires du système de santé $"^{58}$, les infirmières titulaires du master sont amenées à encadrer les équipes soignantes dans des situations de soins dites complexes. Qualifié de "pratique infirmière avancée", ce nouveau rôle ne va pas sans susciter certaines tensions: si ces infirmières bénéficient d'une certaine reconnaissance de la part des médecins, elles se heurtent souvent à une incompréhension de la part de celles qu'elles qualifient d'"infirmières de terrain", et se trouvent dans une relation de concurrence avec les infirmières cadres.

Interrogées sur leurs relations avec les médecins, les titulaires du master évoquent un sentiment de reconnaissance:

"On était toujours quand même sur un niveau un peu inférieur [...] Et là, j'ai l'impression que c'est plus le cas, qu'on peut régater au même niveau. Enfin voilà, ces masters, c'est quand même ce que ça nous apporte aussi à quelque part. C'est quand même une reconnaissance au niveau académique. » Mme Nicole, médecine

Ce sentiment de pouvoir s'ériger en interlocutrices de valeur égale à celle des médecins repose notamment sur un langage qui se manifeste d'abord par une manière légitime de parler:

"On sait quel langage adopter pour être dans la cour des grands j'ai envie de dire. On n'est plus la petite infirmière qui arrive pas à exprimer sa pensée, quoi.» Mme Nicole

${ }^{58}$ https://www.hes-so.ch/data/documents/masters-sciences-infirmieres- 422. pdf, consulté le 7 août 2018 . 
Ce langage se manifeste ensuite par le savoir-faire auquel il renvoie, à savoir la démarche de recherche scientifique qui produit un véritable effet de légitimité. Plus enclines que leurs collègues à déclarer mobiliser "toujours" leurs connaissances en recherche (47,7 vs $13,5 \%)$, les titulaires d'un master se réfèrent à des démarches scientifiques dans leurs discours, ce qui leur confère une meilleure assise face aux médecins:

"C'est comme ça que j'ai pu convaincre les médecins. Typiquement, faut pas rêver, ce sont quand même des scientifiques finalement, et quand on arrive avec des arguments, en disant "ben voilà, trois méta-analyses disent ça, trois machins disent ça, les études randomisées...", et ben ils vous écoutent aussi différemment. " Mme Bourg, EMS

Si ce sentiment d'une reconnaissance par les médecins est unanimement partagé par les infirmières interrogées, la question d'une véritable transformation des rapports sociaux demeure ouverte. D'abord parce que, compte tenu des savoirs mobilisés par ces infirmières, il semble que l'on assiste davantage à "une conversion au paradigme biomédical» (Holmes \& Perron, 2008, p. 407) qu'à la valorisation de savoirs propres reposant sur les théories infirmières (Dallaire, 2015). Ensuite parce que les études de cas d'académisation antérieurs - que ce soit en Italie (SENA, 2017) ou aux États-Unis (JuDD, 2010) - tendent à montrer que les rapports sociaux entre infirmières et médecins continuent d'être marqués par la domination.

Si la possession de ce master confère à ces infirmières un sentiment de plus grande proximité et de meilleure égalité face aux médecins, il institue symétriquement une distance avec les «infirmières de terrain», parfois décrites en des termes condescendants :

"Je trouve que les infirmières sont vraiment dans un truc, nez dans le guidon, avec de la peine à prendre du recul et dire: "Il faut changer pis on y va pis on change". Pour le bien de tout le monde. "Mme Demaya, enseignante HES 
Elles ont aussi le sentiment de se heurter à une certaine incompréhension de la part de leurs collègues, qui peinent selon elles à percevoir l'apport des nouvelles connaissances liées à leur formation:

"Moi j'ai une collègue qui est infirmière qui m'a dit une fois: "Pourquoi tu fais le master, dans ce cas-là, fais médecine!" J'ai trouvé complètement à côté de la plaque qu'une infirmière dise ça.» Mme Demaya

À la meilleure reconnaissance des médecins et à cette distance relative vis-à-vis des infirmières de terrain s'ajoute, pour les infirmières titulaires du master de l'IUFRS, une certaine concurrence avec les cadres ${ }^{59}$, qui voient dans le nouveau statut de clinicienne spécialisée une potentielle remise en cause de leur pouvoir. Les infirmières titulaires du master partagent avec leurs collègues de terrain un regard critique sur les cadres, perçues comme des "auxiliaires de l'administration" (DubET, 2002, p. 223) incarnant des logiques gestionnaires opposées aux valeurs professionnelles (Resenterra, Siggen \& Giauque, 2013). Adoptant un point de vue largement partagé au sein de la profession, Monsieur Sari (oncologie) considère que les cadres ne font plus véritablement partie de la profession du fait de leur éloignement des patients: "T'es pas forcément encore infirmier si tu... oui, t'es infirmier dans le sens que tu gères des infirmières, mais en fait tu donnes pas de soins, donc..." Souvent, les titulaires du master mentionnent ainsi des tensions avec ce groupe, tensions qui semblent résulter de positions mal définies: "par rapport aux cadres [...], globalement l'impression que ça nous donne c'est qu'ils se sentent menacés» (Mme Genoud, médecine). Les enjeux de ces tensions semblent se cristalliser autour de la gestion du temps de travail et de la distribution des tâches dès lors que la prise en charge des patients est en jeu. Monsieur Décoster (psychiatrie) déplore ainsi ce qu’il

\footnotetext{
${ }^{59}$ Nous nous référons ici aux cadres dites «intermédiaires» ou encore «de proximité» : infirmières-cheffes d'unité de soins (ICUS) et, dans une moindre mesure, infirmières-cheffes de service (ICS).
} 
qualifie d' "inféodation" aux cadres, et milite pour la mise en place d'une double hiérarchie, gestionnaire et clinique:

"Le clinicien spécialisé est directement inféodé à l'infirmier chef de service, ce qui à mon sens est une aberration. [...] Les cliniciens spécialisés et les chefs de service devraient avoir une interface qui est décisionnelle pour l'organisation des soins. Et les responsables des soins, la clinique ça doit être les cliniciens. Et les responsables de la gestion des ressources et autres ça doit être les gestionnaires."

Mais le caractère conflictuel de ces relations est toutefois minimisé dans les discours, comme si le temps allait œuvrer dans le sens d'une conjonction de toutes vers l'acceptation des bienfaits de ce master et des ressources qu'il confère à la profession.

En raison de la valeur symbolique de leur diplôme, les infirmières titulaires du master de l'IUFRS incarnent un certain avenir pour la profession. Fortes de leurs savoirs scientifiques, elles ambitionnent de participer activement à la redéfinition de la profession en agissant sur les pratiques et le rôle infirmier, revalorisant ainsi une légitimité professionnelle historiquement déficitaire. Cependant, tant le projet professionnel que les positions institutionnelles de ces infirmières demeurent pour l'heure fragiles.

\section{Encadré 2: Claire Fabre, une "élite infirmière " ${ }^{60}$}

Madame Fabre a 28 ans. Se définissant elle-même comme "studieuse», elle entreprend des études de soins infirmiers directement après sa scolarité et en sort diplômée quatre ans plus tard, à 22 ans. Après seulement deux ans d'activité professionnelle,

${ }^{60}$ Le portrait de Madame Fabre a été publié pour la première fois dans la revue Anthropologie \& Santé (Toffel \& Longchamp, 2017). 
elle commence une formation de praticienne formatrice ${ }^{61}$ avant d'entreprendre un master en sciences infirmières en 2009 à l'université. En attendant de terminer son master et d'exercer comme infirmière clinicienne spécialisée ${ }^{62}$, elle travaille à temps partiel dans un service de médecine d'un hôpital universitaire, où son statut de future détentrice d'un diplôme universitaire lui permet de participer à une recherche.

La trajectoire ascensionnelle de Madame Fabre peut s'expliquer par différents éléments qui renvoient tant à sa trajectoire qu'à un état historique de l'espace infirmier. Son origine sociale d'abord: fille d'un père employé de banque et d'une mère enseignante, Madame Fabre est issue des classes moyennes et a ainsi pu compter sur un soutien familial durant ses études. Sa situation géographique ensuite: résidant dans un centre urbain, le coût d'accès à la formation s'en est trouvé réduit. Son appartenance générationnelle enfin, qui lui offre l'opportunité d'embrasser une formation de niveau académique. Madame Fabre fait ainsi une entrée dans l'espace infirmier par la "grande porte», ce qui contribue sans doute à la rapidité de sa trajectoire professionnelle ${ }^{63}$.

\section{Dispositions dominantes et ambition réformatrice}

Madame Fabre est issue de la filière supérieure des études secondaires. Ainsi, son diplôme de Maturité fédérale lui aurait offert la possibilité de suivre des études de médecine. Ce qu'elle ne manque d'ailleurs pas de relever: "J'ai de toute façon pas essayé de faire médecine alors que j'aurais pu m'y inscrire.» Cette entrée dans l'espace infirmier par la "grande porte» contribue à déterminer ses prises de position, puisqu'elle lui permet de vivre son choix de la profession comme le produit d'une véritable élection. Et, loin de se contenter de vouloir être "proche du patient», comme elle le dit, Madame Fabre semble avoir été motivée dès son entrée dans le métier par un projet réformateur visant à rectifier «l'image de l'infirmière dans la société»:

\footnotetext{
${ }^{61}$ Les praticiens formateurs sont des professionnels de terrain responsables des étudiantes et étudiants durant leurs périodes de formation pratique.

${ }^{62}$ Le rôle des infirmières cliniciennes spécialisées consiste principalement à s'assurer de la qualité des soins prodigués dans un service, notamment concernant la sécurité des patients et l'utilisation efficace des ressources.

${ }^{63}$ Sur l'opposition entre "petite porte" et "grande porte» d'entrée dans un espace social, voir Bourdieu (1989).
} 
"Ce qui me dérangeait, c'était l'image de l'infirmière dans la société, qui est souvent plutôt négative. [...] Je trouve que c'est assez contrasté avec l'image qu'on a du médecin, qui est presque un dieu! Je trouvais que c'était assez extrême les deux images. »

Alors que nombre d'infirmières pâtissent de cette «image négative ${ }^{64}$, Madame Fabre a très vite pour ambition d'agir sur la profession, et ce d'une manière bien précise. Issue des classes moyennes et de la filière supérieure de l'enseignement secondaire, elle ambitionne ainsi de reproduire une position dominante dans l'espace infirmier. Ça n'est d'ailleurs qu'une fois avoir été rassurée sur les possibilités d'évolution professionnelle qu'elle se décide à entreprendre ses études en soins infirmiers:

"Je me suis inscrite dans la première volée HES; donc c'était en 2002. Donc c'était le début. Il n'y avait pas encore de master ou de doctorat en Suisse, on en parlait pas encore et je n'étais pas du tout consciente de qu'est-ce que c'était un Bachelor, un master, un doctorat. Quand je me suis posé ces questions lors de l'entretien de candidature, l'enseignante m'a dit: "Ce n'est pas parce que vous êtes infirmière que vous ne pourrez pas continuer à vous spécialiser, à progresser dans votre profession [...]". Et là, j'ai été plus convaincue, parce que j'ai eu un moment d'hésitation.»

Au final, si elle a choisi la profession d'infirmière en «renonçant» à celle de médecin, Madame Fabre s'est auparavant assurée qu'elle pourrait y occuper une position dominante lui offrant la possibilité de participer au projet réformateur auquel elle aspire.

\section{Capital légitime et stratégies d'inversion de la domination symbolique}

Le capital infirmier en possession des «élites infirmières » bénéficie d'une légitimité qui permet à ses détentrices d'en tirer profit jusque dans les régions les plus médicalisées de l'espace. En témoigne le fait que Madame Fabre n'envisage aucunement d'exercer sa profession à l'extérieur de l'hôpital:

"C'est pas que ça m'attire pas, mais c'est vrai que pour le moment je suis bien dans l'hospitalier. [...] C'est assez effervescent, je veux dire, ça va souvent assez vite, j'aime bien.»

${ }^{64}$ Voir notamment Chaves (2005) et LeRT (1996). 
Dans la lutte visant à inverser la domination symbolique de l'espace infirmier, les "élites infirmières» sont en effet les mieux armées, notamment en raison de la dimension théorique de leur capital qui, si elle peut produire certaines réticences parmi les anciennes générations, tourne cependant très vite en faveur de celles qui possèdent ledit capital. Ainsi, et bien qu'elle déclare que ses collègues ne l'ont pas tout de suite perçue d'«un très bon œil» en raison de sa formation, Madame Fabre n'hésite pas à revendiquer la dimension théorique comme l'un de ses «points forts»:

"Je sais que les premiers mois [...], mes collègues expérimentées, elles me voyaient sûrement pas d'un très bon œil parce qu'il me fallait beaucoup de temps pour faire les choses et pis pour être efficace. Et pis peut-être... peut-être qu'elles disaient que c'était parce que j'étais HES. C'est possible que ce soit le cas, parce que c'est sûr qu'on avait moins de formation pratique; on était plus théorique. Et c'était aussi un de mes points forts d'être théorique.»

La valorisation du «capital infirmier» passe en effet par une entreprise d'inversion de la hiérarchie symbolique, visant à arracher la profession à son statut de mise en œuvre pratique pour la faire accéder au rang d'activité noble sous-tendue par un arsenal conceptuel. Ce qui se manifeste d'abord chez Madame Fabre par un refus de limiter la description de l'activité des infirmières à une succession de «tâches»:

"On pourrait décrire une journée en termes de tâches, mais aussi en termes de processus, de qu'est-ce qu'on met en place; ça dépend... Voilà. Souvent on se limite à décrire la profession infirmière en disant des tâches, et justement c'est quelque chose qui pourrait être réducteur.»

En associant explicitement sa manière d'appréhender la profession à sa formation, Madame Fabre laisse entrevoir un certain clivage générationnel avec les infirmières plus âgées qui, ne disposant pas des «mots» pour décrire le "rôle infirmier», ne peuvent véritablement participer à la lutte de positionnement dans laquelle elle se trouve elle-même engagée:

"[...] le rôle infirmier, tout de ce qui est de l'évaluation de la situation, de déterminer les objectifs avec la personne, de l'accompagner par rapport à ça, et pis de favoriser des résultats positifs, ben ça, personne d'autre [que l'infirmière] peut le faire. [...] Mais souvent, l'infirmière il 
lui manque des mots pour dire ça, déjà. Donc si elle n'a pas de bonnes bases théoriques déjà dans sa formation de Bachelor, elle ne pourra pas dire ça, elle ne pourra pas se positionner.»

L'entreprise d'inversion de la domination symbolique se manifeste encore par la propension de Madame Fabre à se positionner comme alter ego des médecins. On peut remarquer cette conception de leurs rapports dans le regard qu'elle pose sur eux, partant de certaines formations qu'elle et les médecins ont en commun:

"Là, je suis en train de faire un cours sur l'éducation thérapeutique dans le cadre du master, mais je le fais avec des étudiants en médecine. Ben je suis étonnée d'entendre leur discours qui ressemble énormément [...] On pourrait dire que c'est différent; on pourrait dire que souvent l'infirmière a comme objectif d'accompagner, et le médecin c'est souvent de poser un diagnostic et après de soigner, guérir souvent dans le terme guérir. Mais après, quand on discute de manière plus individuelle avec les médecins [...] quand on parle vraiment des motivations profondes, ils ont souvent les mêmes. »

Ramenant les médecins sur «son» terrain - l'éducation thérapeutique étant une formation typiquement infirmière -, elle participe d'une véritable stratégie d'inversion de la domination symbolique, échappant ainsi aux tactiques auxquelles se trouvent souvent réduites les infirmières plus âgées ${ }^{65}$. Elle se distingue par la même occasion des infirmières situées au pôle médical de l'espace, plus enclines à valoriser leur position en situant leurs pratiques sur le même terrain que celui des médecins.

\section{Une réformiste accommodante}

Si l'émergence de la fraction des élites infirmières peut être rapportée aux changements structurels qui caractérisent l'espace infirmier, ces changements doivent à leur tour être inscrits dans

\footnotetext{
${ }^{65}$ Nous reprenons ici la distinction établie par De Certeau (1980) entre stratégies et tactiques: "J'appelle "stratégie" le calcul des rapports de force qui devient possible à partir du moment où un sujet de vouloir et de pouvoir [...] est isolable d'un environnement. Elle postule un lieu susceptible d'être circonscrit comme un propre et donc de servir de base à une gestion de ses relations avec une extériorité distincte [...] J'appelle au contraire "tactique" un calcul qui ne peut pas compter sur un propre, ni donc sur une frontière qui distingue l'autre comme une totalité visible. La tactique n'a pour lieu que celui de l'autre» (p. 21).
} 
l'évolution du champ médical. Nombre d'auteurs s'accordent à penser que la très forte autonomie qu'avait atteinte ce champ durant les Trente Glorieuses tend à s'affaiblir depuis quelques décennies suite à l'intervention de logiques exogènes (ORFÀLI, 2002; PINeLL, 2008). Cette perte relative d'autonomie du champ médical participe sans nul doute à la valorisation du capital infirmier. On sait en effet que la prise de distance face à l'objectif thérapeutique, d'une part suite à l'augmentation des maladies chroniques (Orfàl, 2002), d'autre part liée à la prise en compte de logiques profanes (et notamment de l'avis des familles des patients), fait partie intégrante de ce qu'Anne Paillet (2007) qualifie de «mission» de la profession infirmière. Si Madame Fabre peut ramener les médecins sur son propre terrain, ça n'est donc pas seulement en raison de la position qu'elle occupe, mais aussi parce que ces derniers s'y aventurent plus volontiers que par le passé.

L'apparition des élites infirmières peut donc aussi se lire comme un indice de la relative perte d'hégémonie du pouvoir médical. II convient toutefois de ne pas livrer un bilan unilatéral de ce processus, en rappelant que le pouvoir de réfraction du champ médical est loin d'être épuisé. Ainsi, la formation des élites infirmières n'échappe pas au contrôle du champ médical, puisqu'elle se déroule en son sein ${ }^{66}$. Dans la même veine, il convient de préciser, avec Patrice Pinell (2008), que l'intervention de logiques exogènes au champ médical ne remet pas fondamentalement en question le pouvoir de ses élites, certaines y voyant même l'opportunité de renforcer leur position. De fait, «la question des ajustements nécessaires à "l'évolution de la société" devenue un enjeu dans les luttes de concurrence au sein des élites hospitalières a favorisé l'accession aux positions de pouvoir de nouvelles fractions "réformatrices" qui vont contrôler l'adaptation aux normes imposées de l'extérieur» (p. 63). Et l'on peut se demander si la fraction des élites infirmières n'est pas en passe de se mettre au service, au moins partiellement, de cette nouvelle fraction de l'élite médicale. Alors que les logiques managériales de la nouvelle gestion publique implémentées dans le secteur de la santé entraînent une forte pression sur les soignants (BELORGEY, 2010; GIAUQUe, 2014), on sait par ailleurs que l'accélération de la prise en charge qui en découle est traditionnellement vécue par les infirmières comme une atteinte

\footnotetext{
${ }^{66}$ Les deux Instituts de sciences infirmières des Universités de Bâle et de Lausanne sont rattachés à la faculté de médecine pour le premier et de biologie et médecine pour le second.
} 
à la norme professionnelle d'écoute et d'accompagnement des patients (ACKER, 2005). Or, Madame Fabre fait au contraire preuve d'une certaine complaisance à l'égard de ces changements. Elle présente ainsi les nouvelles formations académiques offertes aux infirmières non pas comme des outils permettant de contester l'ordre établi, mais au contraire comme le meilleur moyen de s'y adapter.

Au final, les élites infirmières apparaissent peut-être comme l'incarnation de l'ambivalence des rapports sociaux entre soins infirmiers et médecine. L'émergence de cette fraction semble résulter à la fois d'une évolution de la structure de l'espace infirmier, de la perte relative d'autonomie du champ médical et de l'effort de ce dernier pour déployer de nouvelles formes d'assujettissement, moins visibles. Si les élites infirmières apparaissent bel et bien comme des réformatrices, ces réformes s'accommodent toutefois pleinement des évolutions en cours du champ médical ${ }^{67}$.

\footnotetext{
${ }^{67}$ Sur les rapports ambivalents qu'entretiennent certaines fractions de la profession avec les réformes du new public management, voir Toffel \& TAWFIK (à paraître).
} 


\section{Chapitre 8}

\section{Les hétérodoxes}

La quatrième et dernière fraction est située en bas à gauche de l'espace (graphique 5). Ces infirmières exercent prioritairement en santé scolaire $(13,5$ vs 4,6$)$, en psychiatrie $(12,4$ vs 7,8$)$, aux soins à domicile (11,8 vs 6,0), en EMS (9,4 vs 5,5) et en établissements socio-éducatifs (5,1 vs 2,29$)$, soit des secteurs le plus souvent extrahospitaliers, et très peu médicalisés et appareillés. Bien que situés au plus bas de l'échelle de prestige, ces secteurs sont néanmoins susceptibles d'offrir de nouvelles opportunités d'autonomie à ces infirmières (A. Hughes, 2010), que ce soit sous la forme du rôle propre fondé sur la relation (LERT, 1996) ou sous celle d'une "régulation offensive" consistant à s'approprier certaines activités jusque-là monopolisées par les médecins (Feroni \& Kober, 1995).

L'examen des indicateurs relatifs au capital mobilisé montre que ces infirmières disposent d'un faible volume de capital à dominante infirmière: rareté de la modalité "toujours» d'une part, faible mobilisation des connaissances en physiopathologie $(9,3$ vs 30,1$)$ et utilisation de machine $(2,9$ vs 27,5$)$ associée à une forte mobilisation des compétences relationnelles (84,9 vs 74,2), des valeurs personnelles (43,0 vs 36,4) et de l'intuition d'autre part (34,6 vs 29,5). Cependant, et à la différence des dominées 
médicales qui peuvent être décrites comme des «dominantes médicales en petit», ces infirmières ne peuvent être considérées comme "démunies». Elles bénéficient en effet d'un fort capital institutionnalisé, avec notamment des formations postgrades académiques $(14,1$ vs 6,7$)$ à orientation psychologie et social $(35,1$ vs 19,4$)$, ou paramédicale et développement personnel $(17,4 \text { vs } 9,2)^{68}$. Dans la même veine, ces infirmières se distinguent par une forte adhésion aux soins non conventionnels, qu'il s'agisse d'assister à des congrès de médecine complémentaire (16,0 vs $5,4)$ et de développement personnel $(32,1$ vs 13,4$)$ ou de délivrer des conseils de médecine complémentaire à leurs patients $(24,0$ vs 7,5 ), conseils qui peuvent à l'occasion aller à l'encontre des consignes de leur employeur $(54,7$ vs 33,0$)$.

Cette série d'indicateurs permet d'esquisser les contours d'une lutte pour une forme de légitimité et d'autonomie professionnelle qui, tout en s'opposant à l'orthodoxie médicale incarnée par les dominantes médicales, se distingue aussi de la lutte menée par les élites infirmières. De fait, ces infirmières constituent sans doute la fraction qui, par sa politisation et sa contestation de l'ordre médical, s'éloigne le plus de la «mobilisation consensuelle» que l'on observe habituellement au sein de la profession infirmière (Sainsaulieu, 2012). Elles peuvent de ce fait être qualifiées d' "hétérodoxes».

\section{Capital importé et mises en œuvre clandestines}

On le voit, le capital infirmier des hétérodoxes présente une forte composante contestataire directement destinée à une prise en charge non médicale des patients et à la dénonciation

\footnotetext{
${ }^{68}$ Précisons également que le revenu équivalent plein temps des hétérodoxes est très proche du revenu moyen (6 417 vs 6401 francs pour une femme de 43 ans). Pour les revenus moyens des différentes fractions non contrôlés par d'autres variables, voir tableau A4 en annexe 3.
} 
des rapports de pouvoir ${ }^{69}$. Cette fraction est ainsi celle qui se distingue le plus fortement des deux fractions du pôle médical, et en particulier des dominantes médicales. C'est peut-être aussi au regard de leur posture critique qu'il faut comprendre la relative indifférence des hétérodoxes à l'égard de la dimension scientifique de la profession ${ }^{70}$, dimension dont on sait qu'elle peut être perçue comme une stratégie servant "d'alibi pour l'inaction dès lors qu'il s'agit véritablement de modifier les relations de pouvoir»(ElzINGA, 1990, p. 172, notre traduction).

Alors que les élites infirmières luttent pour l'autonomie de la profession en développant un capital infirmier à composante scientifique et managériale qui les éloigne de la pratique clinique, et donc de la confrontation directe avec les médecins - ce qui leur permet d'en tirer profit jusque dans les régions les plus médicalisées de l'espace ${ }^{71}$-, les hétérodoxes luttent pour une forme alternative d'autonomie. Pour ce faire, elles mobilisent en particulier des savoirs et savoir-faire exogènes à l'espace infirmier. Exerçant en psychiatrie, Madame Emery a ainsi suivi des cours d'analyse transactionnelle durant quatre ans. Quant à Madame Vexier (oncologie), elle s'est formée à l'homéopathie et au drainage lymphatique. Pour les hétérodoxes, cette forme de capital infirmier manifeste clairement des dispositions subversives relatives au contenu de leur pratique.

\footnotetext{
${ }^{69}$ La dimension politisée du capital infirmier des hétérodoxes se manifeste aussi par leur adhésion massive à l'ASI ( 58,2 vs 33,3\%), association syndicale autant que professionnelle.

${ }^{70}$ Les hétérodoxes ne présentent pas d'adhésion significativement plus forte que l'échantillon à la proposition «La profession infirmière devrait davantage développer un savoir scientifique qui lui soit propre».

${ }^{71}$ Comme en témoigne leur score significativement plus élevé sur l'échelle de médicalisation $(2,07$ contre 1,46), les élites infirmières sont plus souvent en contact avec les médecins que les hétérodoxes. On relèvera par ailleurs qu'elles sont largement mieux représentées que ces dernières dans les secteurs les plus médicalisés de la profession : 12,0 vs $1,2 \%$ en soins intensifs, $11,4 \%$ vs $5,7 \%$ en soins continus, $15,6 \%$ vs $5,0 \%$ aux urgences et $15,1 \%$ vs $1,2 \%$ au bloc opératoire.
} 
Un tel capital est dès lors susceptible de se transformer en une forme de "capital négatif" pouvant à l'occasion se retourner contre celles qui le mobiliseraient de manière trop explicite. Raison pour laquelle ces infirmières optent plus souvent que les autres pour une mise en ouvre clandestine de certains de leurs savoirs et savoir-faire. Plus de la moitié d'entre elles $(54,7 \%$, contre 33,0\% de l'échantillon) déclarent ainsi proposer à leurs patients des soins qui ne respectent pas les consignes de leur employeur ou de leur hiérarchie. Une pratique que Madame Vexier assume pleinement:

"- Ça m'arrive de suggérer à un patient ou à une maman: "Vous devriez amener un peu d'homéopathie. Allez à la pharmacie!"

- Légalement, vous avez le droit de faire ça?

- Absolument pas. Absolument pas.»

Quant à Madame Emery, les mises en garde de son employeur ne l'ont pas découragée à mettre en œuvre ses compétences en analyse transactionnelle:

"Ils m'ont pris en me disant que je ne pouvais pas faire de l'analyse transactionnelle. Ce qui m'a bien fait rigoler parce qu'en fait, l'analyse transactionnelle est un langage: quand c'est intégré, c'est intégré!"

On le voit, les infirmières qui importent des pratiques exogènes afin de constituer un capital spécifiquement infirmier doivent payer de leur personne, que ce soit sur le plan économique (elles financent souvent elles-mêmes leurs formations) ou sur celui de la mise en ouvre de leurs savoirs et savoir-faire qui suppose tout un travail d'invisibilisation. Aux formations "certifiantes" repérées au pôle médical s'opposent donc ici des formations potentiellement "disqualifiantes», au moins aux yeux du pouvoir médical. 


\section{Une hétérogénéité des pratiques}

Les hétérodoxes sont les infirmières qui entretiennent le moins de contacts avec les médecins ${ }^{72}$. Ce qui leur permet d'évoluer dans un environnement peu compétitif: la progression hiérarchique ne fait pas partie des ambitions perçues chez les collègues ${ }^{73}$, et le sentiment d'effectuer des tâches qui devraient normalement revenir à des subalternes est ici moins répandu qu'ailleurs (1,3 vs 3,4). Mais c'est surtout la forte hétérogénéité des pratiques qui frappe: chacune a la possibilité de développer sa propre manière de donner les soins (24,5 vs 14,8), et l'expérience de vie est considérée comme au moins aussi importante que la formation (20,7 vs 14,3$)$. Cette hétérogénéité des pratiques se révèle dans les propos de Madame Maystre qui, exerçant aux soins à domicile, décrit les interventions de ses collègues comme étant fortement marquées par les idiosyncrasies personnelles:

"Moi je sais que j'ai une collègue qui ouvre tout le temps les frigos. Elle c'est son truc, elle va tout le temps penser à ça. Il y a une collègue qui va plutôt regarder les pieds parce que c'est un truc auquel elle pense, et c'est vrai que pour plein de choses comme ça, on a un regard différent."

Cette hétérogénéité se révèle encore dans les critères d'attribution des patients. S'ils suivent des protocoles stricts au pôle médical, ils laissent davantage de place à certaines affinités électives au pôle infirmier. Et notamment en milieu psychiatrique, où Madame Emery, tout en déclarant ignorer les critères mis en œuvre par ses collègues, peut choisir ses patients en fonction de références personnelles:

"Mes collègues, je ne sais pas comment ils font. Mais moi, je sais que je choisis en fonction [...] de ce que je peux faire

\footnotetext{
72 Seules 6,6\% d'entre elles se situent au maximum de notre indice de contacts directs et indirects entre infirmières et médecins (contre 22,4\% pour l'échantillon). ${ }^{73}$ Elles sont $21,7 \%$ à ne "pas du tout" percevoir d'ambition hiérarchique chez leurs collègues (contre $15,6 \%$ pour l'échantillon).
} 
avec ces gens. Parce que si, par exemple, ce sont des dépressifs, et bien je sais que je vais pouvoir faire un monstre travail en analyse transactionnelle. Ça, bon, je choisis, mais je ne le raconte pas."

Un secteur d'activité offre d'autant plus de possibilités de développer un capital spécifiquement infirmier que la concentration de médecins et d'actes médicaux y est moins forte. Mais si cette moindre présence médicale favorise le développement d'un "rôle propre" fondé "sur une approche globale de la personne dans le soin" (LERT, 1996, p. 103), cette voie suppose aussi de renoncer, pour partie au moins, à certains des avantages dont jouissent les infirmières situées au pôle médical. À commencer par la standardisation des pratiques, dont on a vu qu'elle favorisait une certaine assise identitaire.

\section{Une proximité avec les subalternes et les patients}

Parce qu'elles exercent dans des secteurs peu médicalisés et qu'elles visent à développer et mettre en ouvre un capital spécifique, les infirmières du pôle infirmier se caractérisent par un double éloignement, physique et symbolique, à l'égard des médecins. À la différence de leurs homologues du pôle médical, elles ne bénéficient donc que peu de l'autonomie du champ médical, et entretiennent dès lors une certaine proximité avec les subalternes autant qu'avec les patients. Proximité revendiquée, certes, mais qui les expose également à une mise en concurrence, tant sur le plan de la division du travail que sur celui de la définition même de la prise en charge des patients. Pas moins de $28,2 \%$ d'entre elles (contre 16,2\% de l'échantillon) déclarent devoir rappeler souvent ou toujours aux autres professionnels la teneur de leur rôle. C'est ce qu'exprime Madame Gorlier, infirmière à 
domicile, qui se voit contrainte de réexpliquer son "job» à chaque nouvelle aide-soignante:

"Ce qui est difficile à domicile c'est la communication. Parce que moi je vais expliquer bien mon job à une aide, et pis si y en a cinq qui passent chez ce patient, il faudra bien que je le fasse cinq fois quoi. Donc après, il y a des infos qui se perdent.»

Cette exigence de rappel s'explique sans doute, pour partie, par la prise en charge "globale» qui est particulièrement défendue dans cette région de l'espace infirmier, prise en charge qui entraîne une certaine superposition des rôles entre infirmières et aide-soignantes (VASSY, 1999). Exerçant en EMS, Madame Pouli estime ainsi que la toilette des patients ne devrait pas être entièrement déléguée aux aide-soignantes:

"C'est tout à fait légitime que ce soit du personnel infirmier qui fasse la toilette [...] Comme ça, on a vraiment la vision globale du résident, que ce soit de la mobilisation, pour l'alimentation aussi, et puis comment il est capable aussi au niveau cognitif [...] Et on remarque dans l'évaluation d'une aide-soignante, elle n'aura pas des questions aussi pertinentes qu'une infirmière."

Mais alors que, comme nous l'avons montré pour les dominantes médicales, les zones d'indifférenciation peuvent être vécues comme sources de valorisation lorsqu'elles concernent des tâches médicales, les hétérodoxes courent toujours le risque de vivre cette indifférenciation comme une déqualification dès lors qu'elle concerne des "soins de base» habituellement pris en charge par les aide-soignantes. Car dans ces zones de chevauchement, la frontière entre le travail "honorable» et le «sale boulot» peut vite être franchie.

Et l'on comprend que l'apparition de la nouvelle profession d'assistantes en soins et santé communautaire (ASSC) inquiète 
davantage les infirmières du pôle infirmier que celles du pôle médical. D'abord parce que les secteurs les moins médicalisés sont ceux qui ont été les plus prompts à engager ces professionnelles, mais aussi parce que l'arrivée de cette nouvelle catégorie réinterroge la frontière entre les infirmières et leurs subalternes. Madame Pollier précise que les ASSC qui exercent dans son service sont parfois amenées à effectuer certaines tâches traditionnellement réservées aux infirmières, telles que la responsabilité de jour ou l'accompagnement du médecin durant la visite médicale. Des tâches qui ne leur sont pas attribuées dans tous les secteurs: "Ailleurs, dans certains milieux, elles ne sont pas amenées à faire ça, c'est vraiment l'infirmière [qui le fait]». En définitive, la loi de l'espace infirmier veut que les infirmières les moins "protégées" par la possession d'un fort capital médical et d'une forte institutionnalisation de la division du travail soient aussi celles qui côtoient les subalternes les plus qualifiées, et donc les plus «menaçantes» ${ }^{74}$.

À cette proximité avec les subalternes s'ajoute celle avec les patients, en partie revendiquée. Les infirmières hétérodoxes se montrent ainsi les plus ouvertes à intégrer les désirs des patients dans les soins qu'elles prodiguent (46,4 vs 33,1$)$. Une caractéristique d'autant plus révélatrice que leurs patients souffrent le plus souvent de maladies chroniques qui, à la différence des maladies aigües, entraînent "les acteurs de la maladie au-delà du monde médical, dans toutes les sphères de la vie sociale, pour une période de temps indéterminée, mettant en interaction des acteurs de plus en plus nombreux" (BASZANGER, 1986, p. 4). Cette perméabilité à des logiques étrangères au champ médical se manifeste en particulier dans la conception

\footnotetext{
${ }^{74}$ Précisons que cette situation semble être une spécificité helvétique. En France par exemple, le secteur gériatrique est caractérisé par une sous-qualification des aide-soignantes (BAstien \& Rick, 2009). Ce qui n'empêche pas que la distance entre infirmières et aide-soignantes s'y réduise fortement, "de sorte qu'il arrive souvent que les "compétences" de chacun, clairement délimitées dans un service de chirurgie par exemple, soient de plus en plus confuses en gériatrie» (BASTIEN \& Rick, 2009, p. 10).
} 
de la santé à laquelle adhèrent ces infirmières. Interrogées sur les critères qu'elles retiennent pour considérer qu'un patient est en «bonne» ou en "mauvaise» santé, elles tendent à écarter d'emblée toute définition reposant exclusivement sur l'état physiologique, pour lui préférer une définition dans laquelle l'état subjectif apparaît comme le premier des critères:

"La bonne santé, c'est quelqu'un qui arrive à se lever tous les jours avec le sourire, même que vous pouvez être dans une chaise roulante, vous pouvez quand même avoir le sourire [...] Vous avez pu vous recycler, vous avez un métier et vous pouvez quand même faire des choses. Vous pouvez quand même vivre votre vie même que vous êtes en chaise roulante; vous pouvez quand même être heureux. La bonne santé, pour moi, c'est fonctionner... correctement dans la mesure du possible. Vous pouvez avoir des problèmes d'alcool, mais quand même fonctionner correctement parce que vous avez pris conscience qu'il ne faut pas taper vos gosses, qu'il faut vous lever, faire à manger correctement pour vos gamins; vous voyez comment? Il n'y a personne qui, dans la vie, n'a pas de problème, qui n'a rien, qui ne fait face à rien du tout; mais malgré tout on peut quand même être en bonne santé, on peut quand même fonctionner correctement." Mme Emery, psychiatrie

C'est avant tout le fait de "fonctionner correctement», autrement dit de transcender la maladie - et non pas l'absence (au sens clinique) de pathologie - qui fonde ici la «bonne» santé. Et l'on comprend alors que les hétérodoxes soient aussi les infirmières qui se montrent les plus réticentes face à la proposition suivant laquelle les patients ignorent souvent leur véritable état de santé 75 .

\footnotetext{
${ }^{75}$ Près de la moitié (48,7\%, contre 44,1\% de l'échantillon) des hétérodoxes désapprouvent cette proposition.
} 
On le voit, une telle définition écarte d'emblée toute possibilité d'établir un "diagnostic» comme le font les dominantes médicales sur la base d'un savoir dont une catégorie professionnelle détiendrait, si ce n'est le monopole, du moins l'usage. Et l'on est donc bien loin de cette attitude, constatée chez certaines infirmières du pôle médical, consistant à contredire un patient se déclarant en bonne santé. La démarche vise ici tout au contraire à "accompagner» le patient en évitant de lui imposer des définitions étrangères à sa propre perception. Ce que relève Madame Maystre: "On accompagne, on n'est pas une instance supérieure qui vient et puis qui sait tout et puis se la ramène sur tout!»

Une telle conception de la santé et de l'accompagnement des patients n'est bien sûr pas étrangère aux types de pathologie qu'ont à prendre en charge les hétérodoxes, soit le plus souvent des maladies chroniques qui excluent toute possibilité de "guérison". Dès lors, l'une des fonctions du rôle d' "accompagnement» de l'infirmière consiste à opérer chez le patient une sorte de conversion dispositionnelle afin qu'il accepte l'évolution de son état organique. Ce qu'exprime très bien Madame Emery lorsqu'elle considère que l'infirmière est un "agent du mieux-être [...] qui fait que la personne va changer". Et l'on comprend qu'un tel travail d'inculcation suppose un très fort investissement dans la dimension «relationnelle» du soin.

\section{Encadré 3 : Monique Emery, une « hétérodoxe » ${ }^{76}$}

Madame Emery a 55 ans. Fille d'une mère souffrant de troubles psychiques et d'un père inconnu, elle est placée chez ses grandsparents quelque temps après sa naissance. C'est principalement sa grand-mère, couturière de profession, qui s'occupera de son éducation. Infirmier en psychiatrie, son grand-père n'a pas suivi

\footnotetext{
${ }^{76}$ Le portrait de Madame Emery a été publié pour la première fois dans la revue Anthropologie \& Santé (Toffel \& Longchamp, 2017).
} 
de formation particulière: "Il a fait ça à une époque où il n'y avait pas d'école, où en fait on prenait les infirmiers parce qu'ils étaient baraqués.» Madame Emery termine sa scolarité obligatoire en 1976 et entreprend alors une formation d'infirmière-assistante. Plusieurs éléments contribuent à cette orientation. II y a d'abord la pression qu'exerce sur elle le désir d'ascension sociale de sa grand-mère qui coupe court à son souhait initial de devenir courtepointière: "Elle a dit: "C'est exclu, tu ne feras jamais ça!" Ma grand-mère voulait que j'aille à l'université [...] On était un peu en conflit perpétuel.» La formation d'infirmière-assistante, qui ne dure que 18 mois, peut ainsi être considérée comme un moyen de souscrire à moindres frais à cette pression: "Je me suis dit: "Vite fait, bien fait, dix-huit mois, et hop on va faire ça! [...] après j'aurai la paix!"» II faut deuxièmement considérer les antécédents familiaux de Madame Emery qui la mettent en contact direct avec le milieu des soins. Sa mère d'abord qui, souffrant de troubles psychiques, fait des séjours réguliers en milieu psychiatrique; son grand-père ensuite, infirmier en psychiatrie, qu'elle considère comme un "exemple»: "Mon grandpère, c'était un exemple. Mais je pense que ce n'est jamais un hasard si on fait telle ou telle profession [...]. Les chats ne font pas des chiens.»

\section{Une évolution lente}

Dès l'obtention de son diplôme d'infirmière-assistante, Madame Emery éprouve une vive attirance pour la profession: "Ça m'a passionnée; c'est un métier qui m'a passionnée!» Cependant, et malgré cet enthousiasme, son évolution professionnelle est particulièrement lente: elle travaille durant dix-huit ans dans un service de médecine comme infirmière-assistante avant d'obtenir un diplôme d'infirmière. Pour comprendre la lenteur de ce parcours, il faut d'abord considérer que le statut d'infirmière-assistante représente la «petite porte» d'entrée dans l'espace infirmier, ce qui contribue dès le départ à ralentir sa progression. II faut ensuite prendre en compte son lieu d'habitation, une zone périphérique dans laquelle les possibilités pour les infirmières-assistantes d'entreprendre une formation d'infirmière diplômée ne seront mises en place que tardivement. Or, comme Madame Emery élève seule ses trois enfants durant cette période, elle ne dispose ni du temps ni des ressources financières pour effectuer les déplacements qu'aurait exigés une formation située loin de son domicile.

Ce n'est qu'en 2000, soit 24 ans après son entrée dans la profession, qu'interviendra une progression au niveau institutionnel avec un 
diplôme obtenu en soins infirmiers. Elle quitte alors le service de médecine où elle avait exercé jusqu'alors pour se diriger vers un service de chirurgie, soit vers le pôle médical de l'espace infirmier. Elle n'y travaille que quelques années: "Au bout de trois, quatre ans, je me suis dit: "Ou bien je change, je fais quelque chose d'autre, ou bien je reste là jusqu'à la retraite».» Elle décide alors de quitter la chirurgie et hésite entre la pédiatrie et la psychiatrie:

«lly avait deux choses qui m'intéressaient, la pédiatrie et la psychiatrie. J'avais fait un stage de pédiatrie. J'aimais énormément la pédiatrie, mais c'est hyper méticuleux; tous les soins qui sont faits sont vraiment très, très précis à la goutte près; et avec l'âge, la vue qui se taille, ça me faisait peur. Je me suis dit: "Il faut pas se tromper..." Enfin, bref. Ce qui fait que j'ai choisi la psychiatrie parce que ça me passionnait depuis longtemps.»

L'hésitation de Madame Emery entre deux domaines relativement opposés est révélatrice d'un certain clivage dispositionnel: l'attirance pour la technique et le concret liée à ses origines populaires d'une part ${ }^{77}$; l'attirance pour le relationnel, et la psychiatrie en particulier, liée à son héritage familial d'autre part. Son choix, qui se porte finalement sur la psychiatrie, s'explique ainsi par une véritable attirance qui s'est manifestée dès ses premières années comme infirmière-assistante durant lesquelles elle avait déjà entrepris des formations dans ce domaine: "J'ai fait énormément de cours au niveau psy, parce que j'aimais bien le développer. J'ai fait beaucoup de cours de communication, de choses comme ça.» La psychiatrie lui apparaît dès lors comme "le bon plan pour exploiter un peu ça». Mais ce choix relève sans doute aussi d'une nécessité faite vertu qui renvoie encore aux origines populaires de Madame Emery et à ses conditions de vie actuelles. Élevant seule ses trois enfants, elle doit en effet composer avec une double contrainte, celle de diminuer son taux d'activité sans renoncer à son activité professionnelle. Une double contrainte qui, on le sait, n'est que difficilement compatible avec les services hospitaliers, et qui pousse nombre d'infirmières ne pouvant ni continuer à taux plein ni cesser leur activité professionnelle à se diriger vers les secteurs extrahospitaliers.

\footnotetext{
${ }^{77}$ Concernant le lien entre le rapport à la santé et l'appartenance sociale, voir LONGCHAMP (2009).
} 
Au final, on s'aperçoit que les origines populaires de Madame Emery ne sont pas étrangères à sa longévité dans la profession infirmière. Elle observe d'ailleurs elle-même que cette longévité est assez rare:

"Ben là, dans mon unité, il y a Jules, il y avait Françoise; oui, on est deux-trois rescapés de la Grande Ours, mais pas tellement plus. Et à [l'hôpital régional], je pense qu'il y en une dizaine sur deux cents. »

\section{Des formations exogènes}

Présentant une trajectoire semblable à ces oblats "qui, voués dès l'enfance à l'institution scolaire, lui sont totalement dévoués» (BOURDIEU, 1984a, p. 305), Madame Emery accorde une importance primordiale à la formation qui apparaît à ses yeux comme déterminante à tous les niveaux de soins. Cette soif de connaissances, qu'elle a assouvie en suivant tout au long de sa carrière de nombreuses formations en cours d'emploi, s'est cependant souvent heurtée à une offre limitée dans l'espace infirmier et à une absence de reconnaissance de la part de ses employeurs. Durant ses premières années d'exercice comme infirmière-assistante, Madame Emery se voit ainsi contrainte de payer elle-même ses formations: "À cette époque-là, la formation continue, souvent on la payait soi-même. On allait à droite, à gauche faire des cours. " Tout se passe comme si cette situation de relative errance formative révélait un certain décalage entre l'envie de formation de Madame Emery d'une part, et l'état historique d'un espace infirmier qui n'offrait pas encore de formations longues de type académique d'autre part. Ce décalage entre ses aspirations et l'offre de formation, qui renvoie à une appartenance générationnelle, forme l'un des schèmes générateurs des représentations et pratiques de I'«hétérodoxe», puisqu'il pousse ces infirmières à se diriger vers des formations externes à l'espace infirmier. Sitôt son diplôme de soins infirmiers en poche, Madame Emery se lance ainsi dans une formation de quatre ans en analyse transactionnelle, formation qu'elle finance elle-même et qu'elle mène parallèlement à son activité professionnelle.

\section{Dispositions subversives et capital potentiellement disqualifiant}

L'offre limitée de formations au sein de l'espace infirmier d'alors explique en partie le fait que Madame Emery se tourne vers des formations externes à la profession. En partie seulement. Car cette démarche est également sous-tendue par la présence de dispositions subversives qui renvoient, là encore, à l'appartenance 
générationnelle. Parvenue à l'âge adulte durant les années 1970, Madame Emery appartient en effet à une génération sociale marquée par une profonde remise en question du système institutionnel. Tout comme ces «médecins des ouvriers » qui, le plus souvent issus des classes populaires et occupant les positions les plus dominées du champ médical (médecine générale et médecine du travail), défendaient durant les années 1970 une conception militante de leur pratique (Marichalar \& PITTI, 2013), Madame Emery fait montre de fortes dispositions subversives qui s'expriment au travers d'un regard sévère porté sur le système économique et les inégalités qu'il produit.

C'est par exemple par le biais d'une critique radicale du rôle que jouent les assurances maladie que Madame Emery exprime son insatisfaction du système:

"Les assurances, je les porte pas vraiment dans mon cour. Je pense que c'est eux qui nous dirigent maintenant, et c'est pour ça que c'est le bordel absolu quoi. [...] J'ai vu le début de la prise de pouvoir des assurances. [...] Le début, finalement peut-être, de la médecine à deux vitesses, ces choses-là quoi. Clairement une ingérence.»

Et c'est précisément pour lutter contre cette «médecine à deux vitesses» qu'elle a recours à l'analyse transactionnelle fondée par le psychiatre américain Eric Berne. Cette approche lui semble en effet davantage accessible aux patients issus des classes populaires, et ceci dans une double perspective. Sociale d'abord: en tant que vulgarisation de l'analyse freudienne, elle rend abordable une approche conçue par et pour les classes supérieures:

"Si vous analysez les gens qui font de l'analyse [freudienne], ce sont la moitié du temps des profs, des gens qui ont de l'argent [...] Je veux dire, une mère de famille, elle ne pourra jamais faire de l'analyse. II ne faut pas se leurrer. Et ça, Berne, il avait compris ça rapidement [...] [L'analyse transactionnelle] repose sur une philosophie en fait humaniste où il y a un concept d'égalité. Et moi, c'est ça que j'aime. »

À cette accessibilité financière s'ajoute une accessibilité cognitive, rendue possible par des "concepts vraiment très clairs» dont la mise en œuvre, contrairement à celle de l'analyse psychanalytique, produit des effets rapides et visibles: "Quasiment n'importe qui emploie ça [les techniques de l'analyse transactionnelle]. Vous expliquez ça à un patient, en trois secondes il a saisi la combine!» 
Cette mise en œuvre de savoirs exogènes a cependant un coût non négligeable. De fait, ces savoirs ne sont pas nécessairement reconnus par les employeurs, et peuvent même être frappés d'interdiction. Lors de son embauche dans un hôpital psychiatrique, Madame Emery s'est ainsi vu signifier par son employeur qu'il ne souhaitait pas qu'elle ait recours à l'analyse transactionnelle avec les patients. Mais, à l'instar des "médecins des ouvriers» qui effectuaient des contrôles médicaux dans les usines à l'insu des patrons, Madame Emery n'hésite pas à braver cette interdiction, optant pour une mise en œuvre clandestine de cette approche dans laquelle elle a énormément investi ${ }^{78}$.

«Tactique» plus que «stratégique», cette mise en œuvre clandestine révèle le fait que le capital infirmier des hétérodoxes peut fonctionner comme capital potentiellement disqualifiant. Les formations exogènes que suivent ces infirmières s'opposent ainsi à celles qui, nombreuses au pôle médical de l'espace infirmier, sont la plupart du temps financées et reconnues par l'employeur.

\section{Une technicisation du relationnel}

Si les modalités par lesquelles Madame Emery investit sa position professionnelle peuvent s'expliquer pour partie par son appartenance générationnelle, elles semblent également s'expliquer par son origine populaire. Bien qu'elle ait occupé des positions situées aux deux pôles de l'espace infirmier, tout semble indiquer que Madame Emery ait toujours eu une propension à privilégier les dimensions somatiques et techniques plutôt que les dimensions psychiques et relationnelles. C'est ainsi que, décrivant la fonction d'infirmière-assistante qu'elle a exercée dans un service de médecine, elle l'aborde exclusivement sous sa dimension technique:

"C'est une place un peu... bien définie par ce qu'on pouvait faire; on avait le droit de distribuer les médicaments, on avait le droit de faire les injections IM [intramusculaires] et sous-cutanées [...]. Tout ce qui était prise de sang, voie veineuse, tout ça, on n'avait pas le droit de le faire. La définition était claire.»

\footnotetext{
${ }^{78}$ Les entretiens ont révélé d'autres pratiques faisant l'objet d'une invisibilisation par les infirmières, tel le recours à l'homéopathie ou à l'intervention de "guérisseurs".
} 
Plus révélateur encore, Madame Emery semble investir le secteur de la psychiatrie dans lequel elle exerce aujourd'hui avec ces mêmes schèmes techniques. Si elle croit particulièrement en l'efficacité de l'analyse transactionnelle, c'est parce qu'il s'agit selon elle d'une approche très concrète qu'elle compare à une «boîte à outils»:

«Par exemple, vous avez un conflit avec quelqu'un, la plupart du temps, le conflit vient parce que l'autre il se situe en parent, et il vous prend pour un enfant; c'est le même genre de rapport qu'on a à la maison. Papa qui gueule et l'enfant qui dit: "Oui papa." [...] Simplement, en se mettant les deux dans la position d'adulte, le conflit s'enlève. Mais comment se met-on dans la position d'adulte? C'est ce genre de techniques qu'on apprend.»

«Boîte à outils», ensemble de «techniques» permettant de faire changer "très rapidement les choses», l'analyse transactionnelle telle que présentée par Madame Emery semble ainsi répondre à l'une des conditions de la pleine reconnaissance des interventions des professionnels de santé par les membres des classes populaires: le résultat immédiat et visible qui, ramenant «brutalement du côté de la santé et de la vie active» (Boltanskl, 1969, p. 98), permet de constater qu'on en a pour son argent.

Au final, la psychanalyse de Freud et l'analyse transactionnelle de Berne cristallisent une série d'oppositions homologues dans le discours de Madame Emery: Freud/Berne, inaccessible/accessible, lent/rapide, abstrait/concret, toutes les oppositions secondaires pouvant être rapportées à l'opposition fondamentale entre classes supérieures et classes populaires. 


\section{Chapitre 9}

\section{La dynamique de l'espace infirmier}

L'espace infirmier que nous venons de décrire peut être considéré comme une "photographie» de la profession, soit comme une image saisie à un moment donné. Mais cet instantané ne doit pas nous faire oublier la dynamique de cet espace professionnel: la plupart des infirmières s'y déplacent, en exerçant dans plusieurs secteurs tout au long de leur carrière. Il s'agira donc dans ce chapitre de dérouler le «film» de l'espace infirmier, en décrivant les trajectoires professionnelles typiques des infirmières et en identifiant les principales caractéristiques (âge, sexe, nationalité, etc.) qui y sont associées.

Une carrière peut être définie comme le "parcours suivi par une personne au cours de sa vie, et plus précisément au cours de la période de sa vie pendant laquelle elle travaille» (Hughes, 1996, p. 175). En ce qui nous concerne, nous nous concentrons sur les différentes étapes professionnelles des infirmières, comprises entre leur entrée et leur sortie du métier.

Parler de carrière des infirmières suppose de s'intéresser à leur durée. En Suisse, les infirmières qui quittent la profession le font généralement assez tôt dans leur carrière (ADDOR, SCHWENDimanN, Gauthier, Wernli, Jäckel \& Paignon, 2016) et une part importante d'entre elles se reconvertit dans d'autres professions de la santé ou dans des professions du social, de l'éducation, de 
l'enseignement et de l'administration publique (SCHWEndimann, Jäckel, Paignon, Gauthier, Wernli \& Addor, 2016). En France, la durée des carrières des infirmières s'élève à 30 ans dans les hôpitaux publics, 33 ans dans le secteur privé et 35 ans parmi les infirmières libérales (BARLET \& CAVILLON, 2011). Ces résultats remettent en question lidée fortement répandue suivant laquelle les infirmières effectueraient des carrières courtes. La courbe d'âge de notre échantillon semble d'ailleurs confirmer cette longévité des carrières, puisque ce n'est qu'à partir de 57 ans que l'on assiste à une baisse significative des effectifs ${ }^{79}$. On peut donc considérer que, mis à part les quelques départs précoces, les carrières des infirmières sont relativement longues.

Parallèlement à la durée des carrières, il faut s'intéresser aux différents types de trajectoire. Compte tenu de la diversité des secteurs (rappelons que nous en distinguons trente-deux), quels sont les chemins empruntés par les infirmières au cours de leur carrière et quels en sont les déterminants? Afin de répondre à cette question, commençons par considérer l'espace des positions présenté dans le chapitre 3 .

Le graphique 6 présente la position qu'occupe chacun des trente-deux secteurs au sein de l'espace infirmier. Afin de réduire la complexité des trajectoires professionnelles, nous avons regroupé ces secteurs en quatre régions, selon qu'ils sont situés dans le cadran en haut à gauche (quatre secteurs), en haut à droite (six secteurs), en bas à gauche (treize secteurs) ou en bas à droite (neuf secteurs).

Sans surprise, les quatre fractions d'infirmières identifiées dans les chapitres précédents ne se distribuent pas de manière homogène au sein de ces quatre régions, chacune étant surreprésentée dans celle qui correspond à sa position (tableau 5). Aussi, et afin de

\footnotetext{
${ }^{79}$ L'âge moyen d'obtention du diplôme de base s'élevant à 24 ans parmi les infirmières de notre échantillon, la durée moyenne des carrières serait donc de 33 ans en Suisse romande, soit une durée comparable à celle que l'on observe en France. Il faudrait toutefois y retrancher les années d'interruptions professionnelles liées, par exemple, à l'éducation des enfants.
} 
Graphique 6. L'espace des positions et ses différentes régions

Axe 2 - Taux modifiés : 0.23

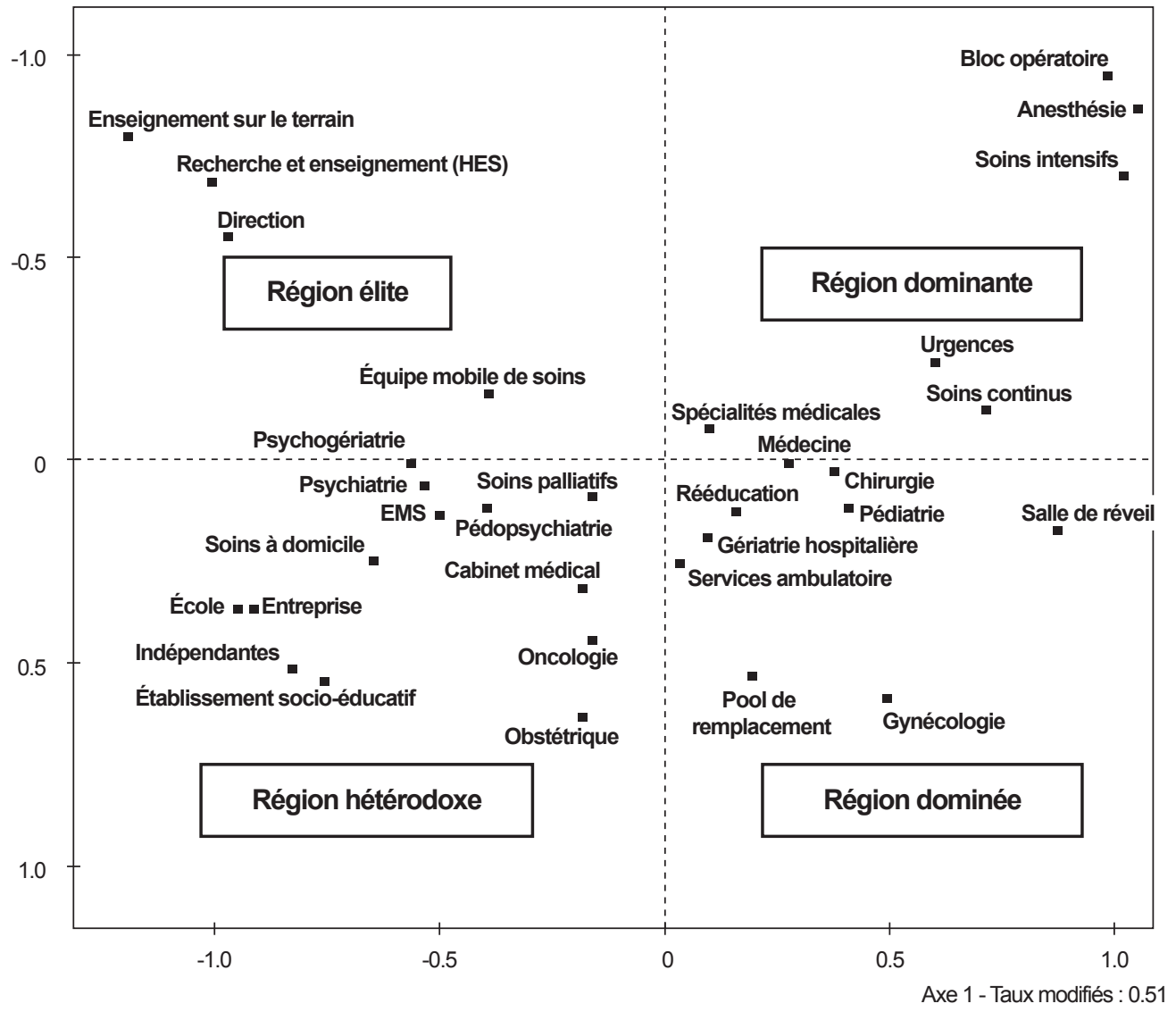

Source: EPRIS.

Note: Les secteurs d'activités ont été projetés en éléments supplémentaires.

Les régions ont été tracées manuellement. $\mathrm{N}=2923$. 
Tableau 5. Répartition des quatre fractions d'infirmières au sein des quatre régions de secteurs

\begin{tabular}{|l|l|c|c|c|c|c|}
\cline { 3 - 7 } \multicolumn{2}{c|}{} & $\begin{array}{l}\text { Région } \\
\text { élite }\end{array}$ & $\begin{array}{c}\text { Région } \\
\text { dominante }\end{array}$ & $\begin{array}{c}\text { Région } \\
\text { hétérodoxe }\end{array}$ & $\begin{array}{c}\text { Région } \\
\text { dominée }\end{array}$ & Total \\
\hline \multirow{2}{*}{$\begin{array}{l}\text { Élites } \\
\text { infirmières }\end{array}$} & $\mathrm{n}$ & 97 & 106 & 265 & 199 & 667 \\
\cline { 2 - 7 } $\begin{array}{l}\text { Dominantes } \\
\text { médicales }\end{array}$ & $\mathrm{n}$ & 58,8 & 16,7 & 25,0 & 18,7 & 22,8 \\
\cline { 2 - 7 } & $\%$ & 3,6 & 530 & 53 & 265 & 654 \\
\hline \multirow{2}{*}{ Hétérodoxes } & $\mathrm{n}$ & 49 & 33 & 463 & 135 & 680 \\
\cline { 2 - 7 } & $\%$ & 29,7 & 5,2 & 43,6 & 12,7 & 23,3 \\
\hline \multirow{2}{*}{$\begin{array}{l}\text { Dominées } \\
\text { médicales }\end{array}$} & $\mathrm{n}$ & 13 & 165 & 281 & 463 & 922 \\
\hline \multirow{2}{*}{ Total } & $\mathrm{n}$ & 7,9 & 26,0 & 26,5 & 43,6 & 31,5 \\
\cline { 2 - 7 } & $\%$ & 100 & 100 & 100 & 100 & 100 \\
\hline
\end{tabular}

Source: EPRIS.

simplifier le propos, nous parlerons dès à présent de «région élite» (en haut à gauche), de "région dominante» (en haut à droite), de «région hétérodoxe» (en bas à gauche) et de «région dominée» (en bas à droite).

Les infirmières de notre échantillon présentent des trajectoires plus ou moins complexes entre ces quatre régions ${ }^{80}$. Certaines (29\%) peuvent être considérées comme "sédentaires", car elles ont toujours exercé dans la région où elles travaillent actuellement. D'autres (42\%) présentent des trajectoires qui impliquent deux régions. D’autres encore (29\%) en présentent des plus complexes impliquant trois, voire les quatre régions ${ }^{81}$.

\footnotetext{
${ }^{80}$ Nous nous basons ici sur la question qui demandait aux infirmières dans quel secteur elles avaient exercé par le passé (elles avaient la possibilité de donner plusieurs réponses).

${ }^{81}$ Précisons que, concernant les trajectoires impliquant plusieurs régions, nous ignorons leur ordre chronologique ainsi que la temporalité séparant les différentes étapes.
} 


\section{Un mouvement général des secteurs hospitaliers vers les secteurs extrahospitaliers}

Selon Everett Hughes (1996), toute profession se caractérise par une combinaison entre une activité centrale et des activités accessoires. L'activité centrale est généralement considérée comme un passage obligé, indispensable à l'acquisition du métier: "on pense qu'il faut avoir fait son apprentissage dans l'activité centrale pour être capable de remplir des fonctions dans l'administration, la formation des générations suivantes, ou la recherche» (p. 183). Une carrière se définit ainsi en partie par une évolution du temps affecté aux différentes activités constitutives d'une profession: on observe typiquement que les individus consacrent davantage de temps à l'activité centrale en début de carrière, pour se consacrer ensuite toujours plus aux activités accessoires, certains abandonnant même complètement l'activité centrale.

Tout indique que les trajectoires infirmières se conforment à un tel modèle. De fait, le travail au chevet du patient en milieu hospitalier apparaît assurément comme l'activité centrale par laquelle l'immense majorité des infirmières (90\%) débutent leur carrière, les activités en milieu extrahospitalier, dans l'administration, la recherche ou l'enseignement apparaissant quant à elles comme des activités accessoires davantage recherchées en seconde partie de carrière. On observe ainsi que les trajectoires allant de la droite vers la gauche de l'espace infirmier (soit des secteurs hospitaliers vers les secteurs extrahospitaliers) sont beaucoup plus fréquentes ( $27 \%$ des infirmières) que celles allant en sens inverse $(10 \%)$. Commencer sa carrière à l'hôpital - et en particulier dans la région dominée ${ }^{82}$ - pour se diriger

\footnotetext{
${ }^{82}$ Près d'une infirmière sur cinq $(16 \%)$ est une «sédentaire de la région dominée». Cette région rassemble par ailleurs les secteurs dans lesquels la proportion d'infirmières occupant leur premier poste est la plus élevée: $23,0 \%$ en chirurgie, $21,4 \%$ en pédiatrie, $19,3 \%$ en médecine et $18,1 \%$ en rééducation. On peut ainsi considérer la région dominée comme la principale "porte d'entrée» de la profession.
} 
ensuite vers les secteurs extrahospitaliers apparaît donc comme une trajectoire typique.

Si le travail hospitalier au chevet du patient est considéré comme l'activité centrale, c'est aussi parce que l'hôpital est le lieu par excellence d'acquisition du capital médical, soit le capital le plus prestigieux de la profession. Alors que les formations d'infirmière contribuent à la valorisation des secteurs de soins aigus et à la dévalorisation correspondante des secteurs de soins chroniques (STEVENS, 2011), exercer directement dans les régions hétérodoxe ou élite au sortir des études semble être une option rejetée par la majorité des infirmières (seules 10\% sont dans ce cas), y compris par celles qui savent qu'elles souhaitent à terme exercer en milieu extrahospitalier. C'est le cas de Monsieur Pichoud qui, bien qu'attiré par la gériatrie, a cependant préféré commencer par la médecine:

"Je me suis dit: "Si je pars tout de suite en gériatrie, je risque de perdre un peu l'aspect aux soins aigus." [...] C'est vrai qu'en gériatrie, il y a beaucoup de soins de base, toilettes, pansements, beaucoup de relationnel, mais peu de pompes ou seringues, défibrillateur, sondes naso-gastriques. [...] Alors, j'ai réfléchi pis j'ai dit: "Bon, ben j'ai meilleur temps d'aller à l'hôpital quelque temps, et pis je verrai, je trouverai ma voie."”

C'est la même démarche qu'a adoptée Madame Maystre, qui exerce actuellement aux soins à domicile:

"J'avais déjà fait un stage à domicile, je savais que ça m'intéressait beaucoup; mais c'est vrai qu'il y a moins de technicité, il y a plus de relation, plus de... de gestion de situation, de réflexion et pis de soins relationnels. Alors je me suis dit: "je vais quand même aller tenter le bien aigu, les soins très techniques", pour voir si ça me plaisait.»

Après deux années d'exercice en médecine, son manque d'attirance pour les soins «techniques» ne fait que se confirmer: "C'était pas 
vraiment le truc qui me bottait.» Elle décide donc de quitter le milieu hospitalier pour se diriger vers les soins à domicile. Mais ce départ précoce (elle n'est alors âgée que de 23 ans $^{83}$ ) vers l'un des secteurs les moins prestigieux de la profession ne passe pas inaperçu, et Madame Maystre doit affronter le regard sceptique et parfois méprisant de ses collègues de médecine:

"Dans la profession, comment c'est vu les CMS?

- C'est vu comme la planque pour ne pas travailler [en souriant].

- C'est vrai?

- Ouais. Passablement. Quand j'ai dit que je venais ici [aux soins à domicile], j'étais à [l'hôpital] encore: "Pourquoi tu vas travailler là-bas?"

- Et vous en pensez quoi, vous?

- Je pense que c'est pas vrai, mais... Après, ça peut donner une image, parce que...

- Vous allez boire des cafés chez les gens, quoi.

- Voilà! Non mais c'est vrai, on a du temps avec les gens et pis aussi, ben on court pas pour poser 18 perfusions, enfin des choses comme ça." Mme Maystre, soins à domicile

Bien qu'habituelle, la sortie de l'hôpital ne doit donc pas se faire de manière trop précoce. Ni de manière trop abrupte: rares, très rares même $(1,4 \%)$, sont les infirmières qui empruntent la trajectoire diagonale reliant directement la région dominante à la région hétérodoxe. La transition par la région dominée apparaît donc comme un passage quasi obligé pour les infirmières des secteurs les plus médicalisés souhaitant rallier les secteurs extrahospitaliers. Il existe ainsi une véritable étanchéité entre la région la plus médicalisée et la région la moins médicalisée de l'espace infirmier.

${ }^{83}$ Les infirmières des régions dominante et dominée sont nettement plus jeunes (40 ans en moyenne) que celles des régions élite et hétérodoxe (46 ans). 


\section{Une dissociation entre trajectoires professionnelles et trajectoires sociales}

Reste à se demander quels sont les éléments qui tendent à expliquer ces trajectoires. D'une manière générale, trois dimensions déterminent les carrières professionnelles: l'âge biologique, l'âge social et le changement historique (Hughes, 1996).

Le changement historique se rapporte aux évolutions structurelles qui entraînent la disparition de certaines tâches ou professions au profit de nouvelles. Dans le cas de la profession infirmière, ce changement se manifeste par le processus de différenciation horizontale et verticale décrit en introduction: la multiplication des postes extrahospitaliers ainsi que l'académisation de la formation offrent de nouvelles opportunités de carrières pour les jeunes générations d'infirmières, en même temps qu'elles tendent à dévaluer certains postes et formations désormais considérés comme "anciens». Si ce changement historique constitue l'arrière-fond de notre recherche, il n'en forme cependant pas l'objet. Nos analyses se concentrent davantage sur les effets de l'âge biologique et de l'âge social, auxquels nous avons ajouté la dimension de genre qui, bien que faiblement thématisée par Everett Hughes, nous semble incontournable du fait de la composition majoritairement féminine de la profession.

Afin d'identifier les facteurs qui tendent à influencer les carrières professionnelles, concentrons-nous sur les neuf trajectoires les plus fréquentes - soit celles qui impliquent les régions dominante, dominée et hétérodoxe - représentées dans le graphique $7^{84}$. On y distingue trois trajectoires «sédentaires» (représentées par les flèches circulaires), deux trajectoires «ascendantes» (celles qui convergent

\footnotetext{
${ }^{84}$ Ces neuf trajectoires sont obtenues en éliminant toutes celles associées à la région élite (trop peu d'infirmières concernées) ainsi que toutes celles qui combinent plusieurs positions passées (dont l'interprétation est rendue difficile en raison de l'absence d'informations relatives à leur ordre chronologique). Ces neuf trajectoires comptabilisent 1995 individus (68,2\% de l'échantillon).
} 
vers la région dominante), deux trajectoires «descendantes» (celles qui émanent de la région dominante) et deux trajectoires «latérales» (celles qui relient les régions hétérodoxe et dominée).

Graphique 7. Neuf trajectoires professionnelles révélatrices

Axe 2 - Taux modifiés : 0.23

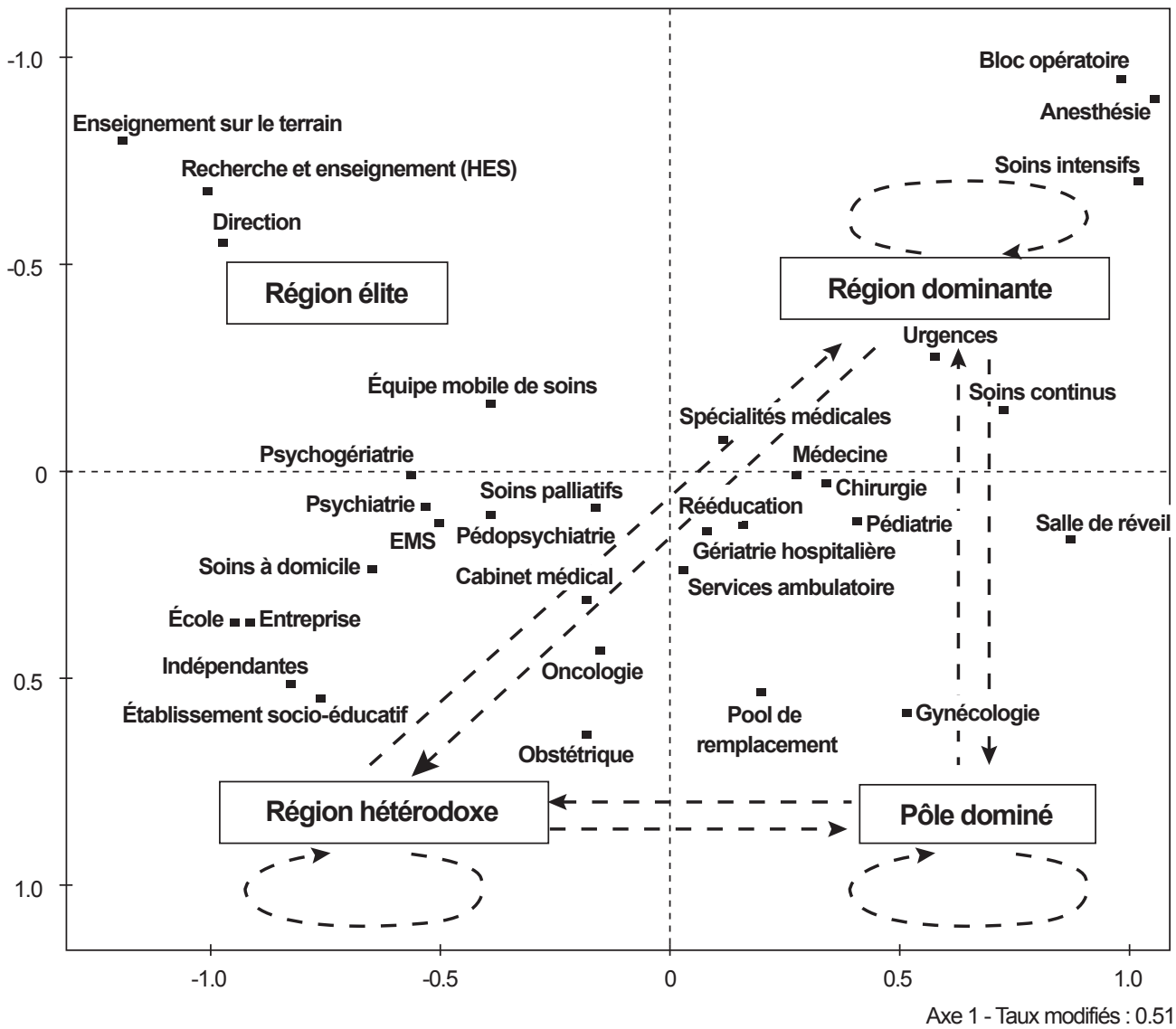

Source: EPRIS. 
En accord avec ce que relève Hughes, l'âge biologique est la dimension qui exerce le plus d'effet sur les trajectoires professionnelles des infirmières ${ }^{85}$. Alors que les plus jeunes se retrouvent davantage chez les sédentaires des régions dominée et dominante ou empruntent les trajectoires menant à ces deux régions, les plus âgées empruntent les trajectoires de sens inverse: celles qui vont vers le bas et vers la gauche de l'espace. L'avancée en âge entraîne donc une désertion des régions les plus médicalisées et de l'activité principale de la profession, à savoir le travail au chevet du patient en milieu hospitalier. Ce qui s'explique, nous l'avons vu, par le fait que l'hôpital constitue un "passage obligé» en début de carrière, une étape qui permet l'acquisition de capital médical. Mais cela s'explique sans doute aussi par le besoin des infirmières plus âgées d'alléger la pénibilité physique caractéristique du milieu hospitalier, qu'il s'agisse de la charge physique ${ }^{86}$ ou des horaires irréguliers. Il n'est à ce titre pas étonnant de constater que le taux d'activité, qui renvoie également à la pénibilité physique, suive la même tendance que l'avancée en âge: alors que les trajectoires menant vers la région dominante sont associées aux taux les plus élevés, la trajectoire allant de la région dominée à la région hétérodoxe est associée au taux le plus faible. La sortie de l'hôpital semble ainsi associée à un certain "désengagement" professionnel ${ }^{87}$.

\footnotetext{
${ }^{85}$ Les résultats qui suivent sont issus d'un modèle de régression multinomiale incluant neuf variables (âge, sexe, nombre d'enfants, nationalité, niveau de formation postgrade, taux d'activité, statut de cadre, revenu du partenaire et position sociale du partenaire). Cette régression, qui inclut 1413 individus, permet de dégager les différentes propriétés sociales des infirmières associées aux trajectoires professionnelles. Le détail du modèle de régression est disponible sur demande auprès des auteurs.

${ }^{86}$ La charge journalière d'une infirmière exerçant en milieu hospitalier peut être estimée à 1000 kilogrammes (Peneff, 1992).

${ }^{87}$ Selon Pao-Long Chang, Ying-Chyi Chou et Fei-Chun Cheng (2006), quatre phases peuvent être distinguées dans la carrière des infirmières: la "phase d'exploration» (2 premières années de pratique), la "phase d'établissement" (entre 2 et 5 ans de pratique), la "phase de maintenance" (entre 5 et 15 ans de pratique) et la "phase de désengagement" (au-delà de 15 ans de pratique).
} 
S'agissant d'une profession fortement féminisée, on pourrait s'attendre à une forte influence du nombre d'enfants sur les trajectoires ${ }^{88}$. Les résultats montrent toutefois que celle-ci n'est que très relative. S’il apparaît que les trajectoires se dirigeant vers la région hétérodoxe sont effectivement associées à un nombre d'enfants plus élevé, cette relation s'estompe pourtant dès lors que l'on contrôle le nombre d'enfants par d'autres variables: toutes choses égales par ailleurs, seules les rares infirmières qui empruntent la trajectoire menant de la région dominante à la région hétérodoxe se distinguent par un nombre d'enfants plus élevé que les autres. Tout semble donc indiquer que le nombre d'enfants produit une forme d'effet "retard et radical» sur les infirmières qui exercent dans la région dominante: si ledit effet ne se fait sentir que tardivement (lorsque le nombre d'enfants est beaucoup plus élevé que la moyenne), il pousse en revanche ces infirmières à quitter brutalement le milieu hospitalier et à emprunter la trajectoire menant directement à la région hétérodoxe sans transiter par la région dominée.

La deuxième dimension qui détermine les carrières professionnelles est ce que Hughes (1996) qualifie d' «âge social». Certains individus tendent en effet à délaisser l'activité centrale de leur profession pour se diriger vers une "voie qui confere prestige, revenus substantiels et réputation»(p. 183). Et il arrive alors que ces individus nourrissent un certain sentiment de supériorité par rapport à ceux qui n'empruntent pas la même direction: "il peut arriver que ceux qui n'abandonnent pas l'activité centrale soient regardés de haut par ceux qui l'ont délaissée»(p. 183). Un tel phénomène se vérifie-t-il dans le cas de la profession infirmière? Si la sortie de l'hôpital s'accompagne

\footnotetext{
${ }^{88}$ Everett Hughes (1996) considère que l'éducation des enfants fait partie d'une série d'éléments qui renvoient au vieillissement biologique et qui influence la carrière des femmes en particulier. Il reconnaît cependant qu' "il n'est pas aisé de distinguer ce qui est une conséquence directe des faits biologiques de ce qui relève des tabous et des définitions sociales" (p. 179).
} 
en effet d'une augmentation du revenu, cette dernière s'explique avant tout par l'ancienneté des infirmières qui exercent dans les secteurs extrahospitaliers. Quant au prestige, nous avons vu que c'est l'activité centrale qui s'en trouve auréolée, soit la pratique clinique au chevet du patient dans les secteurs les plus médicalisés. De ce point de vue, tout rapprochement de la région hétérodoxe s'accompagne d'une baisse de prestige. Mais il faut cependant tenir compte du fait que les trajectoires s'accompagnent bien souvent d'une progression du niveau de formation et d'un changement de statut professionnel. Qu'elles soient issues de la région dominante ou de la région dominée, les infirmières qui se dirigent vers la région hétérodoxe se distinguent ainsi par un niveau de formation beaucoup plus élevé que les autres, notamment en ce qui concerne les formations académiques. Ces mêmes infirmières, ainsi que les sédentaires de la région hétérodoxe, accèdent par ailleurs beaucoup plus souvent que celles des régions dominée et dominante à des postes de cadres ${ }^{89}$. Tout se passe ainsi comme si la perte de prestige professionnel résultant de l'éloignement des secteurs hospitaliers se voyait compensée par une certaine ascension sociale symbolisée par l'accumulation de capital culturel et l'accession aux postes de management. La profession infirmière présente ainsi un paradoxe: une ascension dans l'espace social peut s'accompagner d'un déclin dans l'ordre symbolique proprement professionnel.

Une telle dissociation entre trajectoires professionnelles et trajectoires sociales semble s'expliquer avant tout par la dimension genrée du métier - soit une dimension que Hughes ne fait qu'évoquer. Relevons d'abord que les hommes présentent une faible probabilité d'emprunter la trajectoire allant de la région dominée à la région hétérodoxe, soit la voie principale par laquelle

\footnotetext{
${ }^{89}$ Alors que le taux moyen d'infirmières cadres s'élève à $17 \%$, il est beaucoup plus élevé parmi certains secteurs de la région hétérodoxe: $43 \%$ en psychogériatrie et $48 \%$ en EMS.
} 
les infirmières quittent le milieu hospitalier. Ils tendent ainsi à demeurer sur la droite de l'espace infirmier, ne contribuant que faiblement au mouvement général allant du pôle médical au pôle infirmier ${ }^{90}$. Mais les effets du genre se révèlent pleinement dès lors que l'on prend en compte le statut social et le revenu du partenaire des infirmières: alors que les trajectoires qui mènent vers la région dominante sont empruntées par les infirmières dont le partenaire présente la position sociale la plus basse, les trajectoires qui mènent vers la région hétérodoxe sont au contraire empruntées par celles dont le partenaire présente la position sociale la plus élevée.

Quant au revenu des partenaires, sa prise en compte réduit celui des infirmières au statut de revenu d'appoint: à l'échelle de notre échantillon total, les infirmières présentent en effet un revenu inférieur à celui de leur partenaire ${ }^{91}$. Ce statut de revenu d'appoint se trouve encore renforcé par le fait que les infirmières tendent à diminuer leur taux d'activité - et donc leur revenu - à mesure que s'élève le salaire de leur partenaire ${ }^{92}$. Une tendance qu'il faut rapporter à leur statut de femme plutôt qu'à celui d'infirmière. Pour preuve: les hommes infirmiers échappent complètement à une telle logique ${ }^{93}$. Et c'est donc bel et bien la composition genrée de la profession qui se trouve au principe de sa position dominée.

\footnotetext{
${ }^{90}$ Relevons que les infirmières étrangères tendent aussi à demeurer sur la droite de l'espace infirmier.

${ }^{91}$ Alors que le revenu mensuel moyen des infirmières s'élève à 5190 francs (voir chapitre 2), celui de leur partenaire s'élève à 5631 francs.

${ }^{92} \mathrm{La}$ corrélation entre le revenu des infirmières et celui des partenaires est de $-0,087$ ( $\mathrm{p}=0,001)$. Celle entre le taux d'activité des infirmières et le revenu des partenaires est de $-0,222(\mathrm{p}=0,000)$.

${ }_{93}$ Alors que les femmes de la profession présentent un revenu nettement inférieur à celui de leur partenaire (4 996 vs 5922 francs), les hommes de la profession présentent au contraire un revenu largement supérieur à celui de leur conjointe (6 164 vs 4443 francs). Par ailleurs, et contrairement à ce que l'on observe parmi les femmes, le taux d'activité et le revenu des hommes de la profession ne présentent aucune corrélation avec le revenu de leur partenaire.
} 
$\mathrm{Au}$ final, tout se passe donc comme si les infirmières se trouvaient condamnées à devoir choisir entre ascension dans l'ordre symbolique infirmier et ascension sociale, tout gain sur l'une de ces échelles étant compensé par une perte sur l'autre. Les trajectoires qui mènent vers la région dominante de l'espace infirmier ont ainsi d'autant plus de probabilités d'être empruntées par des infirmières qui cumulent les caractéristiques sociales des dominées de l'espace social: jeunesse, nationalité étrangère, absence de statut de cadre, célibat ou partenaire associé aux classes populaires et moyennes. À l'inverse, les trajectoires qui mènent vers la région hétérodoxe (soit la moins prestigieuse d'un point de vue professionnel) sont empruntées par des infirmières qui, tout en réduisant leur taux d'activité, semblent entamer une ascension dans l'espace social, avec notamment l'accès à des postes de cadre sous-tendu par des formations académiques, la nationalité suisse et un partenaire appartenant aux classes supérieures. La diminution du prestige professionnel qu'impliquent ces trajectoires se voit ainsi doublement compensée: par un allègement de la pénibilité physique d'une part, par une forme d'ascension sociale d'autre part. 


\section{Partie III}

\section{Au cœur de l'ethos professionnel}

En révélant l'existence de différentes fractions aux représentations et pratiques spécifiques, la notion d'espace infirmier nous a permis de présenter la diversité qui caractérise la profession infirmière. Mais cette différenciation ne doit cependant pas faire oublier les dénominateurs communs à toutes les infirmières. Il existe en effet certains enjeux qui, tels la «dimension relationnelle» des soins ou le caractère éminemment humaniste de la profession, demeurent largement partagés au sein de la profession.

C'est à la description de ce véritable ethos professionnel, compris ici comme le "dénominateur commun à un ensemble d'individus pratiquant une activité similaire qui se reconnaissent et sont reconnus comme membres d'un groupe professionnel» (Fusulier, 2011, p. 104) qu'est consacrée cette troisième partie. Nous y montrons la permanence de certains mythes fondateurs et fédérateurs de la profession qui se révèlent notamment à l'examen des motivations sous-jacentes au choix professionnel ou à celui de la distinction entre les dimensions «technique» et «relationnelle» de l'activité.

Nous adoptons cependant ici une posture critique. Il ne faut en effet pas oublier que "l'ethos professionnel relève d'un 
segment social et, par ce fait même, il n'est pas sans rapport avec des ethos plus transversaux: de genre ou de position en particulier" (Fusulier, 2011, p. 105). Nous montrons ainsi que toutes les infirmières n'adhèrent pas avec la même ferveur à l'ethos professionnel infirmier. L'analyse des effets de caractéristiques telles que le genre, l'origine sociale ou encore la nationalité révèle non seulement des adhésions plus ou moins fortes, mais aussi l'existence de certaines minorités professionnelles qui, prenant leurs distances avec certains mythes fondateurs - les dimensions humaniste, vocationnelle et religieuse -, engagent un véritable processus de "normalisation" de la profession. L'analyse critique des termes employés par les infirmières pour désigner les valeurs qui les réunissent montre aussi que ces termes peuvent parfois recouvrir des acceptions distinctes selon les contextes professionnels, certains dénominateurs communs n'ayant alors de commun que le nom. 


\section{Chapitre 10}

\section{Les motivations sous-jacentes au choix professionnel}

La dimension "humaniste» de la profession fait sans doute partie des éléments les plus constitutifs de l'ethos infirmier. La question des motivations sous-jacentes au choix professionnel est à cet égard révélatrice: les motivations se référant à des valeurs humanistes sont en effet les plus mentionnées. Mais cela ne doit pourtant pas faire oublier que toutes les infirmières n'adhèrent pas à ces valeurs avec la même force. En témoigne le fait que le choix professionnel peut être motivé par des considérations beaucoup plus pragmatiques ou opportunistes. L'origine sociale, le sexe et la nationalité des infirmières permettent de rendre compte de cette adhésion plus ou moins forte à l'ethos infirmier.

Il existe de nombreuses études portant sur les motivations qui sous-tendent le choix professionnel des infirmières. Et toutes aboutissent au même résultat: le choix d'exercer la profession repose en premier lieu sur des motivations de type "humaniste». Se référant à des valeurs telles que l' «altruisme», la "noblesse», le "prendre soin», la «compassion» (Price, McGillis Hall, Angus \& Peter, 2013) ou encore la «bienveillance» de la figure nourricière (PRICE, 2009), une telle motivation renvoie bien souvent à une image traditionnelle et quelque peu stéréotypée de 
la profession. Dans une recherche menée auprès d'étudiantes et d'étudiants en soins infirmiers, Mary Mooney, Michele Glacken et Frances O’Brien (2008) montrent ainsi que le besoin intrinsèque de "prendre soin» ("to care») représente la principale motivation avancée pour justifier le choix professionnel. Et en dépit de leur décalage partiel avec la réalité des soins, les motivations de type humaniste ne se tarissent pas avec l'expérience, comme le montre une étude longitudinale également menée auprès d'étudiantes et d'étudiants (ANDERSSON, 1993).

Nos résultats sont pleinement convergents avec ces études: invitées à se prononcer sur une liste de douze motivations, près de neuf infirmières sur dix déclarent que c'est la dimension «relationnelle» qui les a motivées à exercer ce métier, et près de huit sur dix déclarent avoir embrassé la profession «afin de venir en aide aux personnes en souffrance» (tableau 6). Mais bien qu'elles soient les plus plébiscitées, ces motivations humanistes ne sont cependant pas les seules. On retrouve ainsi une forte adhésion à la dimension technique des soins - dimension dont nous verrons plus loin qu'elle forme un couple indissociable avec la dimension relationnelle puis, pour un peu plus de la moitié des infirmières, la motivation que l'on pourrait qualifier de "vocationnelle». Notons encore que près d'une infirmière sur cinq a choisi la profession suite à la maladie d'un membre de sa famille ou d'un proche.

Les différentes motivations que notre questionnaire nous a permis d'identifier peuvent être regroupées en quatre grandes aspirations sous-jacentes: "humaniste», "antivocationnelle», "promotionnelle» et "second choix ${ }^{94}$. Celle «humaniste» regroupe les motivations traditionnellement rattachées à la profession infirmière: "pour venir en aide aux personnes en souffrance", "la dimension relationnelle», "suite à la maladie d'un membre de ma famillelproche» et "partir à l'étranger»" 95.

\footnotetext{
${ }_{94}$ Ce regroupement résulte d'une analyse factorielle.

${ }^{95} \mathrm{Il}$ peut sembler surprenant que le souhait de partir à l'étranger soit associé à une motivation de type humaniste. Ceci se comprend pourtant
} 


\begin{tabular}{|c|c|c|c|}
\hline & $\begin{array}{l}\text { Ne s'applique } \\
\text { pas du tout/ } \\
\text { pas vraiment }\end{array}$ & $\begin{array}{l}\text { S'applique } \\
\text { partiellement/ } \\
\text { tout à fait }\end{array}$ & $\mathbf{N}$ \\
\hline $\begin{array}{l}\text { C'est la dimension } \\
\text { relationnelle qui m'a } \\
\text { motivée à devenir infirmière }\end{array}$ & 10,6 & 89,4 & 2848 \\
\hline $\begin{array}{l}\text { J'ai souhaité devenir } \\
\text { infirmière afin de venir } \\
\text { en aide aux personnes } \\
\text { en souffrance }\end{array}$ & 20,5 & 79,5 & 2820 \\
\hline $\begin{array}{l}\text { C'est la dimension technique } \\
\text { qui m'a motivée } \\
\text { à devenir infirmière }\end{array}$ & 28,4 & 71,6 & 2810 \\
\hline $\begin{array}{l}\text { Aussi loin que je me } \\
\text { souvienne, j'ai toujours } \\
\text { souhaité devenir infirmière }\end{array}$ & 48,3 & 51,7 & 2856 \\
\hline $\begin{array}{l}\text { J'ai souhaité devenir } \\
\text { infirmière pour partir } \\
\text { à l'étranger (CICR, ONG, etc.) }\end{array}$ & 63,3 & 36,7 & 2805 \\
\hline $\begin{array}{l}\text { J'ai souhaité devenir infirmière } \\
\text { pour accéder rapidement } \\
\text { à l'indépendance économique }\end{array}$ & 71,8 & 28,2 & 2786 \\
\hline $\begin{array}{l}\text { J'ai souhaité devenir } \\
\text { infirmière pour faire carrière }\end{array}$ & 78,1 & 21,9 & 2783 \\
\hline $\begin{array}{l}\text { C'est suite à la maladie } \\
\text { d'un membre de ma famille/ } \\
\text { proche que j'ai souhaité } \\
\text { devenir infirmière }\end{array}$ & 81,8 & 18,2 & 2791 \\
\hline $\begin{array}{l}\text { Je n'avais pas d'idée } \\
\text { de métier, alors j'ai choisi } \\
\text { infirmière }\end{array}$ & 84,7 & 15,3 & 2777 \\
\hline $\begin{array}{l}\text { Je souhaitais faire médecine, } \\
\text { mais j'ai échoué la formation } \\
\text { ou mes diplômes ne m'en } \\
\text { donnaient pas la possibilité }\end{array}$ & 90,4 & 9,6 & 2792 \\
\hline $\begin{array}{l}\text { Je souhaitais faire une autre } \\
\text { profession de la santé, } \\
\text { mais j'ai échoué } \\
\text { aux examens d'admission }\end{array}$ & 91,6 & 8,4 & 2790 \\
\hline $\begin{array}{l}\text { Ce sont mes parents } \\
\text { qui souhaitaient } \\
\text { que je devienne infirmière }\end{array}$ & 92,9 & 7,1 & 2784 \\
\hline
\end{tabular}

Source: EPRIS. 
L'aspiration "antivocationnelle» réunit les trois motivations sans lien direct avec la profession: "je n'avais pas d'idée de métier", "pour accéder rapidement à l'indépendance économique» et "ce sont mes parents qui souhaitaient que je devienne infirmière", auxquelles s'ajoute, avec une corrélation négative, la motivation vocationnelle "aussi loin que je me souvienne, j'ai toujours souhaité devenir infirmière». L'aspiration "promotionnelle» comprend l'item "pour faire carrière" ainsi que la "dimension technique». La raison pour laquelle ces deux motivations sont associées n'est pas évidente, mais on peut toutefois poser qu'elles regroupent un même schème générateur dans lequel la technique apparaît comme attribut indissociable du pouvoir. Enfin, l'aspiration "second choix" réunit les motivations qui renvoient à l'échec d'un premier choix ("faire médecine» et "faire une autre profession de la santé»).

On le voit, si les motivations de type humaniste sont les plus plébiscitées, elles coexistent cependant avec des motivations plus pragmatiques. Ce qui nous mène à poser deux questions illustrées dans la figure 1 .

Figure 1. Caractéristiques sociales, motivations, positions et prises de position

\begin{tabular}{|c|c|c|c|}
\hline $\begin{array}{c}\text { Caractéristiques sociales } \\
\text { des infirmières }\end{array}$ & $\longrightarrow$ & \begin{tabular}{|l|} 
Motivations à l'origine \\
du choix professionnel
\end{tabular} & $\begin{array}{c}\text { Positions et prises } \\
\text { de position professionnelles }\end{array}$ \\
\hline
\end{tabular}

Premièrement, ces motivations sont-elles influencées par des caractéristiques sociales antécédentes au choix professionnel, telles que l'origine sociale, le sexe et la nationalité? Deuxièmement, ces motivations produisent-elles à leur tour des effets sur la position occupée au sein de l'espace infirmier, et donc sur les prises de

lorsque l'on considère l'intitulé exact de notre question qui, par la mention d'organismes tels le CICR ou des ONG, exclut d'emblée le voyage de type "touristique» au profit d'une intervention auprès de populations en détresse. 
position y associées? En réinscrivant les agents professionnels dans l'espace social global, de telles questions permettent de saisir tant les modalités d'entrée dans le métier que les manières de l'exercer (Pichonnaz \& Toffel, 2018).

\section{L'influence des caractéristiques sociales sur les motivations}

Nous l'avons dit, les motivations de type vocationnel sont les plus caractéristiques de la profession. Davantage mentionnées par les femmes que par les hommes ${ }^{96}$, elles renvoient à l'image d'une infirmière au service de personnes dans le besoin. Ce type de motivations semble reposer sur une disposition empathique profonde, souvent acquise précocement à l'occasion d'un événement douloureux tel que la maladie d'un proche:

"Ben moi, ce qui m'a motivée dès le début, c’est que j'avais un frère qui était gravement malade. Donc déjà depuis que j'avais 4 ans je pense, j'étais déjà ici [à l'hôpital X], parce qu'il était hospitalisé. Et je pense que ça a déjà influencé mon choix, parce que j'étais déjà en contact avec les hôpitaux dès mon enfance. Donc là, il y avait plus quelque chose d'un sentiment, d'une intuition, que c'est quelque chose que j'avais envie de faire par rapport à... je sais pas moi, des odeurs que je me souvenais de l'hôpital, par rapport à l'ambiance qui y régnait, par rapport à l'image que j'avais de l'infirmière, de ce que j'en ai retenu, de mon expérience personnelle.» Mme Fabre, médecine

"-Quand je suis sortie de mon école obligatoire, je me destinais déjà dans une filière pour devenir infirmière. Donc plus ou moins à l'adolescence, le choix s'est fait.

\footnotetext{
${ }^{96}$ Ces résultats sont issus de quatre régressions linéaires prenant le sexe, l'origine sociale, et la nationalité pour variables indépendantes et le type de motivation pour variables dépendantes (cf. tableau A8 en annexe 3).
} 
Aussi, ben, parce que j'étais confrontée au monde de la santé en étant moi-même hospitalisée, en ayant mes grandsparents hospitalisés, et voilà. C'est un peu ça qui a été le déclencheur, j'ai été faire un stage, ça m'a plu et j'ai choisi cette filière-là.

- Vous dites que vous avez été hospitalisée, c'était dans quel...?

- C'était une histoire d'orthopédie, mais c'est vrai qu'après, dans le contact des infirmières, je trouvais que c'était une profession qui alliait bien, et le côté humain, en même temps il y a du côté technique, c'est varié, ça bouge bien. Enfin ce côté-là c'est ce qui m'a attirée. Mais le côté, ouais, relations humaines. » Mme Garcin, soins continus

Cette motivation de type humaniste peut notamment se manifester par le désir de partir à l'étranger afin de travailler dans le domaine de l'aide humanitaire:

"C'était ça l'idée en fait: c'était d'être infirmière, mais de pouvoir effectuer des missions et voyager dans le monde. C'était ça. C'était plutôt avec l'aide humanitaire on va dire. [...] Je crois que je me voyais sur le terrain avant d'être à l'hôpital, c'est clair, ouais.» Mme Morier, foyer pour requérants d'asile

Relativement éloignées de cette motivation humaniste, celles "antivocationnelle» et de «second choix» caractérisent avant tout les individus qui, en raison de leur sexe ou de leur origine sociale, n'avaient qu'une faible probabilité d'exercer la profession. Les infirmières issues des classes moyennes et supérieures adhèrent ainsi plus souvent à ces deux motivations que celles issues des classes populaires, et les hommes y adhèrent plus souvent que les femmes.

Fille d'une mère enseignante du secondaire et d'un père ingénieur, Madame Dunod (médecine) présente typiquement une motivation que l'on peut qualifier d'antivocationnelle. Occupée à limiter son investissement dans les études en dépit des attentes de 
ses parents, ce n'est que tardivement que son choix professionnel s'est porté sur la profession infirmière:

"J'ai fait l'école de commerce [...], ça c'était trois ans à l'école; après j'ai fait une année de maturité professionnelle [...]. Et puis, je me souviens qu'il y avait eu les dix ans de l'école de soins infirmiers à Delémont. Avec mon cousin, on est allés visiter l'expo et je m'étais dit: "Quelle horreur, je ne pourrais jamais faire ça!" J'étais dans un âge où j'avais pas forcément envie d'étudier. Donc j'avais choisi l'école de commerce parce que là on peut faire un prolongement, et passer par la suite au Lycée. C'est la voie longue ça s'appelle: on fait une année de plus que l'école de commerce et on peut se rallier. [...] Sous l'influence de mes parents, j'avais ça en disant que peut-être tout d'un coup en cours de route, je ferais quand même ça. [...] J'étais vraiment... je savais pas du tout quoi faire et puis je sais pas vraiment exactement pourquoi, j'avais déjà été intéressée par faire droguiste et puis après je me suis dit: "Non, on doit travailler le samedi, je veux pas." [...] Et puis après, à un moment donné, j'ai fait un stage [en soins infirmiers], après deux stages; et puis ça m'a vraiment... c'était ça quoi!; après j'ai plus jamais lâché le truc quoi!»

Quant à Monsieur Bonvin (soins palliatifs), issu d'une famille socialement favorisée et fortement versée dans la santé (une mère assistante dentaire, un père dentiste et une sœur médecin), ce n'est qu'après avoir connu un échec à l'examen d'entrée de l'école de TRM (technicien en radiologie médicale) qu'il s'est intéressé à la profession infirmière. Il s'agit donc clairement d'un "second choix" qu'il qualifie lui-même de choix "par défaut»:

"- Pouvez-vous nous raconter comment vous êtes devenu infirmier; comment ça s'est passé, depuis le départ?

- Euh... par défaut. Au départ, j'avais beaucoup de peine à trouver une idée de métier [...]. Et puis euh... [...] ma 
sour qui est médecin me disait: "TRM ça pourrait être intéressant" [...] Et puis, $j$ 'ai été faire un stage, j'ai dit: "Ok, j'y vais!" Et puis [...] j'ai été malheureusement en échec; j'avais malgré tout déjà signé un contrat pour l'école de radiologie. Il fallait faire un préstage, je crois trois mois, comme aide-infirmier et j'avais déjà signé le contrat; donc ben voilà, j'ai assumé; et là, j'ai été encadré par une infirmière assistante qui finissait sa formation, qui avait besoin d'avoir quelqu'un d'hyper zélé pour lui faire passer ses examens. Et puis là-dedans, elle m'a tellement bien formé [...] qu'elle m'a passé un tout petit peu... ça m'a donné envie. Et en même temps, je dois avouer, si je dis "par défaut", c'est parce qu'à l'époque ça m'arrangeait bien d'avoir quelque chose."

À l'opposé des infirmières issues des classes moyennes et supérieures dont le choix professionnel semble résulter d'une sorte d'errance, on trouve celles qui, issues des classes populaires, parlent de leur choix professionnel comme une manière de destin auquel elles se sentaient vouées dès le plus jeune âge: "Ben déjà pendant les études, je savais déjà que ce serait quelque chose dans le milieu euh... médical» (Mme Jacques, soins intensifs). Quant aux infirmières étrangères, elles présentent tout comme les hommes et les infirmières issues des classes moyennes et supérieures une affinité avec les motivations «antivocationnelle» et de "second choix». Mais s'y ajoute également celle "promotionnelle», fortement caractéristique de ce groupe, ce qui permet de penser que ces infirmières ne sont pas nécessairement représentatives des infirmières de leur pays d'origine (la France dans plus de $80 \%$ des cas), mais qu'il s'agit plus sûrement d'un "sous-échantillon biaisé» (PASSERON, 2006, p. 219) issu d'un recrutement historiquement situé privilégiant les infirmières les plus "carriéristes». 


\section{L'influence des motivations sur les positions professionnelles}

Reste à se demander si les différentes motivations qui sous-tendent les choix professionnels ont une influence sur les positions occupées au sein de l'espace infirmier et donc, indirectement, sur les prises de position associées. Les résultats montrent que ni la motivation "second choix" ni la motivation "humaniste» n'exercent d'influence significative sur l'appartenance aux quatre fractions ${ }^{97}$. La première sans doute en raison du nombre peu élevé d'infirmières concernées (moins de 10\% d'entre elles souhaitaient exercer la profession médicale ou une autre profession de la santé), la seconde probablement en raison de son caractère très consensuel: la motivation humaniste jouit d'un tel succès qu'elle n'exerce pas d'effet clivant sur les positions occupées.

Les motivations «antivocationnelle» et "promotionnelle» sont en revanche des éléments prédictifs des positions occupées. Ainsi, le fait de présenter une motivation "antivocationnelle» diminue significativement la probabilité d'appartenir à la fraction des dominées médicales. Autrement dit, les infirmières de cette dernière fraction sont celles qui ont le plus souvent embrassé la profession par "vocation", soit une motivation davantage plébiscitée par les classes populaires, les femmes et les infirmières de nationalité suisse. Quant à la motivation "promotionnelle», elle opère une claire distinction sur le deuxième axe de l'espace: alors que les infirmières qui adhèrent le plus à cette motivation occupent les positions dominantes, celles qui y adhèrent le moins occupent au contraire les positions dominées.

\footnotetext{
${ }^{97}$ Les résultats sont issus d'une régression logistique. L'effet des quatre motivations sur l'appartenance aux fractions est contrôlé par l'origine sociale, le sexe, la nationalité et l'âge (voir tableau A9 en annexe 3).
} 
La motivation "humaniste» demeure clairement la plus répandue, et en particulier parmi les femmes. Le consensus qui la sous-tend est si fort qu'elle ne détermine aucun profil infirmier spécifique. La motivation "antivocationnelle» est privilégiée par les infirmières issues des classes moyennes et supérieures, par les hommes et par les infirmières de nationalité étrangère, soit celles qui présentent les caractéristiques sociales dominantes. De manière cohérente, les infirmières fortes d'une telle motivation sont largement sous-représentées parmi les dominées médicales. Celles qui adhèrent à la motivation "second choix" présentent les mêmes caractéristiques que celles de la motivation précédente, à savoir qu'elles sont plus souvent issues des classes moyennes et supérieures, plus souvent de nationalité étrangère, et plus souvent de sexe masculin. Mais à la différence de la motivation précédente, embrasser la profession par «second choix" ne mène pas à occuper une position plutôt qu'une autre. La motivation "promotionnelle» enfin, est plébiscitée par les infirmières de nationalité étrangère, et mène à occuper une position de dominante médicale ou d'élite infirmière.

En posant la question des motivations à l'origine du choix professionnel à des infirmières qui pour certaines exercent depuis plusieurs décennies, notre enquête s'expose au risque de l'illusion rétrospective. Nos résultats montrent pourtant que les motivations mentionnées par les infirmières récemment diplômées ne diffèrent pas de celles exprimées par les infirmières plus expérimentées ${ }^{98}$. Ce qui laisse entendre que ces motivations, dont la constance durant les études en soins a déjà été démontrée

\footnotetext{
${ }^{98}$ Une comparaison des réponses aux douze motivations du questionnaire entre les infirmières ayant leur diplôme de base depuis deux ans ou moins d'une part, celles l'ayant depuis 3 ans ou plus d'autre part, permet d'exclure un effet d'illusion rétrospective: les deux groupes présentent des réponses identiques au seuil de significativité de $5 \%$. La seule exception concerne la motivation carriériste, davantage plébiscitée par les jeunes diplômées. Il n'est toutefois pas possible de déterminer si cette différence est liée à un effet d'âge ou à un effet de génération.
} 
par ailleurs (ANDERsson, 1993), demeurent extrêmement stables dans le temps, n'étant soumises ni à un effet d'âge ni à un effet de génération. Et sauf à supposer que la profession infirmière serait substantiellement associée à une motivation humaniste - supposition que la minorité d'infirmières ne se reconnaissant pas dans cette motivation suffit à démentir -, il faut se résoudre à constater que nous avons affaire à un puissant effet de période qui touche simultanément toutes les générations successives: la profession infirmière n'est pas encore sortie de son «âge humaniste». 



\section{Chapitre 11}

\section{Une profession comme les autres? Vision désenchantée et origine de classe}

L'examen des motivations à l'origine du choix professionnel a montré que l'ethos infirmier demeure fortement marqué par des valeurs humanistes et par un sentiment de vocation. Mais il a aussi révélé l'existence de valeurs concurrentes de type plus pragmatique, telles que le "second choix» ou le désir de promotion. Pour poursuivre cette piste d'analyse, nous proposons d'élargir le questionnement en nous intéressant non plus uniquement aux motivations professionnelles initiales, mais également à des prises de position sur le métier ou encore à des données objectives telles que l'héritage familial.

Pour ce faire, nous distinguons ici une conception «traditionnelle» et une conception "progressiste» de la profession. La conception "traditionnelle» se réfere à une infirmière de sexe féminin, dont le choix professionnel serait de type "vocationnel»: s'inscrivant dans un certain héritage familial (par exemple l'un des deux parents ayant exercé une profession de la santé; un proche victime de maladie; une tradition religieuse), le souhait d'exercer la profession se serait imposé dès l'enfance. La conception "progressiste» vise au contraire à «normaliser» la profession infirmière, notamment par l'ouverture à la gent masculine, par 
le rejet de la "vocation" et du bénévolat, et par la constitution d'un référentiel scientifique plutôt que religieux ou spirituel. En reprenant une expression que l'on doit à Max Weber et largement diffusée au sein des sciences sociales, nous qualifions une telle vision de la profession de "désenchantée ${ }^{99}$.

Qu'en est-il de ce processus de normalisation aujourd'hui? La conception "enchantée» de la profession a-t-elle complètement cédé la place à une vision "désenchantée»?

\section{Un processus de normalisation largement entamé}

Examinons d'abord le rôle de l'héritage familial. La proportion d'infirmières dont la mère ou le père exerce ou a exercé une profession de la santé s'élève à respectivement 12,5 et $4,2 \%{ }^{100}$. Cela dit, seules $7,1 \%$ des infirmières déclarent avoir choisi leur profession parce que leurs parents le souhaitaient, proportion à peine plus élevée $(7,9 \%)$ parmi celles dont la mère exerçait le métier. Autrement dit, qu'il prenne la forme d'une filiation ou d'encouragements explicites, l'héritage familial ne concerne qu'une minorité d'infirmières. Cet héritage prend en revanche plus souvent la forme d'un événement familial marquant: $18,2 \%$ des infirmières déclarent ainsi avoir choisi la profession suite à la maladie d'un membre de leur famille ou d'un proche.

S'agissant de la "vocation", la mention de ce terme semble provoquer une réaction plutôt défiante: seules 7,2\% des

\footnotetext{
${ }^{99}$ Max Weber (2003) parle de "désenchantement du monde» à propos du processus de rationalisation par la science et la technique qui accompagna la philosophie des Lumières.

${ }^{100}$ Plus précisément, 7,3\% des infirmières de notre échantillon ont une mère qui a elle-même exercé le métier d'infirmière (1,0\% pour les pères) et 5,2\% ont une mère qui exerçait une autre profession de la santé tel que médecin, sage-femme, aide-hospitalière ou aide-infirmier (3,2\% pour les pères).
} 
infirmières se déclarent en accord complet avec la proposition suivant laquelle "la profession infirmière est une vocation" tandis que $37,1 \%$ se disent en désaccord complet avec cette proposition. La dimension vocationnelle se révèle pourtant dès lors qu'elle est abordée de manière moins directe, avec un peu plus de la moitié des infirmières $(51,7 \%$ ) qui déclarent avoir souhaité exercer le métier depuis leur plus jeune âge.

En ce qui concerne la dimension religieuse enfin, plus de la moitié des infirmières (54\%) déclarent n'appartenir à aucune religion ou confession particulière, et près d'un quart $(23,9 \%)$ estiment que leur degré de religiosité ou de spiritualité est nul, contre 4,2\% seulement se situant au degré maximum.

Ce bref état des lieux permet de constater que le processus de normalisation de la profession est largement entamé: une très grande majorité d'infirmières est issue d'un milieu familial sans lien avec la santé du côté parental, et les encouragements à exercer le métier demeurent relativement rares, y compris lorsque la mère exerçait la profession. Le terme de "vocation" est largement rejeté, et la dimension religieuse ou spirituelle ne constitue de loin pas un socle identitaire pour la profession. La précocité avec laquelle apparaît le souhait d'exercer la profession chez beaucoup d'infirmières s'explique donc sans doute moins par un héritage familial ou une affinité religieuse que par la visibilité sociale de la profession - visibilité que la maladie d'un membre de la famille ou d'un proche ne fait que renforcer.

\section{Désenchantement et origine sociale}

Si la vision désenchantée de la profession est relativement répandue, un sous-groupe se distingue toutefois par une posture plus radicale: formant près de $30 \%$ de notre échantillon, ces infirmières répondent systématiquement par la négative aux quatre questions relatives à l'héritage familial et à la 
vocation ${ }^{101}$. Qui sont ces infirmières qui incarnent pleinement la «normalisation» de leur profession?

Il convient d'abord de relever qu'un tel rapport désenchanté à la profession n'est associé ni à la position occupée au sein de l'espace infirmier (ces infirmières pèsent le même poids - environ $30 \%$ - au sein des quatre fractions), ni à la nationalité, ni à l'âge. Autrement dit, il ne s'explique pas par une quelconque "culture nationale» ou un effet générationnel, et ne mène pas à privilégier une position professionnelle plutôt qu'une autre.

Ces infirmières se distinguent en revanche par leur genre (elles représentent près de la moitié de la population masculine, contre seulement un quart de la population féminine), par leur origine sociale (elles composent un tiers des infirmières issues de classes moyennes ou supérieures, contre seulement un quart de celles issues des classes populaires), par leur degré de religiosité ou spiritualité (elles composent un tiers des infirmières se déclarant très peu ou pas du tout religieuses ou spirituelles, contre seulement un cinquième de celles qui se déclarent très religieuses ou spirituelles) ${ }^{102}$.

Tout semble en fait indiquer que le rapport plus ou moins «enchanté» qu'une infirmière entretient avec la profession est directement lié à la probabilité qu'elle avait d'exercer cette profession. Alors que l'on connaît la concordance des attentes

${ }^{101}$ Réagissant à la proposition suivant laquelle "La profession infirmière est une vocation", ces infirmières se situent à moins de 3 sur une échelle allant de 0 "pas du tout d'accord" à 6 "tout à fait d'accord». Dans la même veine, elles répondent "ne s'applique pas du tout» ou "ne s'applique pas vraiment" aux trois propositions: "Aussi loin que je me souvienne, j'ai toujours souhaité devenir infirmière», "C'est suite à la maladie d'un membre de ma famille/proche que j'ai souhaité devenir infirmière", et "Ce sont mes parents qui souhaitaient que je devienne infirmière».

${ }^{102}$ Le caractère déterminant du degré de religiosité, du genre et de l'origine sociale est confirmé par une régression logistique retenant l'âge, la nationalité et la position au sein de l'espace infirmier comme variables de contrôle (voir tableau A10 en annexe 3). 
subjectives et des probabilités objectives (Bourdieu, 1974), il n'est en effet pas étonnant de constater que le sentiment de "vocation" sous-tendu par les encouragements parentaux s'observe le plus souvent parmi les femmes d'une part, parmi les infirmières issues des classes populaires d'autre part, soit parmi celles pour qui l'accès à la profession est non seulement plus probable qu'ailleurs (une comparaison entre les origines sociales des infirmières de notre échantillon et celles de la population résidente suisse montre que la probabilité pour un individu d'exercer la profession est d'autant plus faible que ses parents occupent une position sociale élevée), mais représente aussi une forme d'ascension sociale propice aux visions enchantées ${ }^{103}$. Et c'est à l'inverse parmi les infirmières issues des classes moyennes et surtout des classes supérieures, soit parmi celles pour qui l'accès à la profession est le moins probable et synonyme de stagnation ou de déclin social, que s'observe la plus forte part des professionnelles désenchantées.

\section{Une « avant-garde désenchantée »}

Si la part des infirmières désenchantées croît à mesure que s'élève l'origine sociale, c'est pourtant parmi celles issues de la fraction socioculturelle des classes moyennes que l'on retrouve la concentration la plus élevée. Identifiée par Daniel Oesch (2006), cette fraction de classe représente $6,9 \%$ de la population active

\footnotetext{
${ }^{103}$ L'ascension sociale des infirmières issues des classes populaires se manifeste encore par leur forte propension à considérer leur poste actuel comme étant favorable à leur progression professionnelle $(77 \%$, contre $70,6 \%$ parmi celles issues des classes supérieures), et par l'optimisme qu'affichent celles issues des fractions les moins qualifiées relativement à leurs perspectives professionnelles (sur une échelle de 0 à 6 , leur moyenne se situe à 4,28, contre 3,74 parmi les fractions socioculturelles des classes supérieures). Un optimisme qui s'exprime malgré des conditions de travail globalement moins favorables, qu'il s'agisse de l'accès aux formations post-graduées, du taux d'activité, de la pénibilité des horaires ou des charges physiques.
} 
en Suisse. Sa localisation (au sein des classes moyennes salariées), sa composition professionnelle (santé, éducation, travail social et culture) et genrée (une majorité de femmes) ainsi que son soutien massif aux valeurs de la "nouvelle gauche» (égalité des droits selon le genre, la «race» et l'orientation sexuelle; protection de l'environnement) en font une fraction en tous points semblable à la "petite bourgeoisie nouvelle» identifiée par Pierre Bourdieu (1979a) dans la France des années 1970.

Près de la moitié des infirmières issues de cette fraction de classe sont des désenchantées, contre un quart seulement parmi celles issues des classes populaires. Un tel résultat peut paraître surprenant, dans la mesure où ces infirmières se trouvent dans une situation de forte reproduction sociale intergénérationnelle (près de $80 \%$ d'entre elles ont au moins un parent ayant exercé une profession dans le domaine de la santé, dans celui de l'enseignement ou dans celui de l'assistance sociale et spirituelle) et semblent ainsi déroger à notre hypothèse d'un désenchantement associé aux trajectoires sociales déclinantes. Mais cette trajectoire de pente nulle est cependant trompeuse, et apparaît comme déclinante sitôt que l'on tient compte du capital culturel des parents: comparées aux infirmières issues des autres fractions des classes moyennes, celles issues de la fraction socioculturelle présentent bien plus souvent un père ou une mère de niveau universitaire ${ }^{104}$. Or l'on sait qu'un tel déclassement culturel est susceptible d'entraîner chez les individus une "lutte contre le déclassement» (Bourdieu, 1978) qui se traduit notamment par le choix de professions peu définies se laissant facilement adapter à leurs dispositions subversives et rétives à toute idée de hiérarchisation d'une part, par une soif de formations en cours d'emploi d'autre part (Bourdieu, 1979a).

\footnotetext{
${ }^{104}$ Les taux de pères et de mères universitaires s'élèvent à respectivement 15,7 et $9,3 \%$ parmi ces infirmières, contre $2,4 \%$ parmi celles issues des classes moyennes indépendantes ou des autres classes moyennes salariées.
} 
La forte concentration de désenchantées parmi ces infirmières trouve une explication supplémentaire dans les caractéristiques culturelles liées à leur origine sociale. On sait en effet que la fraction socioculturelle des classes moyennes se caractérise par un soutien massif aux valeurs dites de la "nouvelle gauche». Héritières de ces valeurs, ces infirmières présentent ainsi une probabilité élevée d'incarner l'avant-garde du processus de normalisation de la profession, notamment par un rejet de tout ce qui évoque "la sacro-sainte chape de plomb qui pèse sur [les infirmières]: l'obéissance, la soumission, la charité et le dévouement de la religieuse» (CALBÉRA, 2003, p. 56). Dans cette perspective, le faible degré de religiosité ou de spiritualité ${ }^{105}$ affiché par ces infirmières doit être considéré comme l'une de leurs caractéristiques majeures.

Nos résultats montrent que l'ethos infirmier traditionnel (qui privilégie la part féminine de la profession, les motivations de type vocationnel, un héritage familial pouvant prendre la forme d'une filiation ou d'encouragements explicites, et une certaine proximité avec la religion) ne constitue plus un véritable socle identitaire professionnel, une partie importante des infirmières n'y adhérant pas. Une telle vision enchantée de la profession est particulièrement rejetée par les infirmières issues des classes moyennes et supérieures, soit par celles pour qui la profession renvoie à une forme de stagnation ou de déclin social. On peut ainsi distinguer deux groupes infirmiers: celles issues des classes populaires, qui adhèrent davantage à l'ethos infirmier; et celles issues des classes moyennes et supérieures, qui la rejettent.

D'autres recherches historiques ou sociologiques ont mis en évidence l'existence de deux groupes distincts sous le

$\overline{105}$ Sur une échelle de 0 à 6 , le degré de religiosité/spiritualité des infirmières issues de la fraction socioculturelle des classes moyennes s'établit à 1,6, contre 2,1 pour l'échantillon. 
rapport de l'origine sociale au sein de la profession infirmière. Joëlle Droux (2000) montre ainsi que la lutte qui oppose, durant la première moitié $\mathrm{du} \mathrm{xx}^{\mathrm{e}}$ siècle en Suisse romande, les filières de formation de la Croix-Rouge Suisse à celle de l'Alliance Suisse des Garde-Malades recouvre une lutte des classes, les premières recrutant exclusivement parmi les femmes de classes moyennes et supérieures, alors que l'Alliance recrute avant tout parmi les hommes de classes populaires. Plus proche de nous, Christian de Montlibert (1980) montre comment la spécialisation de puéricultrice est divisée entre les «modernistes» issues de la petite bourgeoisie d'ascension d'une part, et les "traditionnalistes» issues de la bourgeoisie en déclin d'autre part. Quant à Geneviève Picot (2005), elle montre que les jeunes générations d'infirmières françaises introduisent de nouveaux types de rapports sociaux en raison du fait qu'elles sont à la fois plus masculinisées et davantage issues des classes populaires. Et c'est finalement à Danièle Senotier (1992) que peut être attribué le propos le plus fort sur la question, elle qui n'hésite pas à considérer l'hétérogénéité des origines sociales des infirmières comme "l'un des clivages les plus importants de la profession» (p. 24).

Nos résultats nous mènent à confirmer ces analyses tout en les nuançant. Relevons d'abord que la vision "progressiste» semble avoir changé de camp: autrefois portée par les infirmières issues des classes populaires (Droux, 2000) ou, plus tard, de la petite bourgeoisie d'ascension (De MontLIBERT, 1980), elle se retrouve aujourd'hui parmi celles issues des régions intermédiaires et supérieures de l'espace social, et en particulier parmi les individus subissant un déclin social sur le plan du capital culturel. Mais si cette vision progressiste a changé de camp, on peut toutefois relever une continuité: elle est toujours le fait des infirmières issues des groupes sociaux qui, d'un point de vue statistique, avaient la probabilité la plus faible d'embrasser la profession. Ici comme ailleurs, ce sont les groupes sociaux sous-représentés qui constituent une véritable force d'innovation. 
Le deuxième élément qu'il convient de souligner est que, bien que les infirmières issues des classes moyennes et supérieures soient davantage enclines à la normalisation de la profession que celles issues des classes populaires, l'opposition entre ces deux groupes ne peut en aucun cas être considérée comme "l'un des clivages les plus importants de la profession". Nous avons en effet montré que les principaux clivages professionnels sont déterminés par les positions occupées au sein de l'espace infirmier, et que ces positions, définies par le volume et la structure des formes de capital, demeurent indépendantes de l'origine sociale. Autrement dit, si l'origine sociale produit un effet déterminant sur le rapport plus ou moins enchanté qu'une infirmière entretient avec son activité, cet effet n'affecte que de manière marginale les principales prises de position relatives à la définition légitime des soins, à la nécessité de développer un savoir propre ou encore au positionnement à adopter face aux patients et aux autres professionnels.

\section{Encadré 4 : Déclassement culturel et désenchantement. Le cas de Madame Maceira}

Deux stratégies de lutte contre le déclassement s'offrent aux individus dont les diplômes ne permettent pas de renouer avec la position sociale de leurs parents: ils peuvent «ou bien produire de nouvelles professions plus ajustées à leurs prétentions [...] ou bien aménager conformément à leurs prétentions, par une redéfinition impliquant une réévaluation, les professions auxquelles leurs titres leur donnent accès» (Bourdieu, 1978, p. 14). Nos analyses statistiques ont permis de montrer que les infirmières issues de la fraction socioculturelle des classes moyennes sont particulièrement prédisposées à la deuxième stratégie: alors que leur diplôme en soins infirmiers est souvent synonyme de déclassement culturel par rapport à la formation de leurs parents, elles représentent l'avantgarde du processus de «normalisation » de la profession en rejetant tout ce qui se rapporte de près ou de loin à l'idée de "vocation». Par la mise à distance de tous les carcans liés à la tradition, une telle vision «désenchantée» leur permet de réévaluer la profession 
conformément à leurs aspirations culturelles et, par là, de renouer partiellement avec leurs origines.

Le cas de Madame Maceira est particulièrement révélateur de cette situation. Issue d'une famille de classes moyennes à fort capital culturel, rien ne la prédisposait à exercer les soins infirmiers dont elle raille même les aspects les plus traditionnels. Son choix de la filière psychiatrique et sa mise à distance du corps des patients et de leurs émanations; son insistance sur l'importance du raisonnement infirmier et son rejet corrélatif de toute idée de subordination; son abandon de l'activité clinique au profit de postes de formation et de direction; l'extension revendiquée du domaine d'intervention des soins infirmiers et le brouillage des frontières interprofessionnelles qui en résulte: tout dans sa posture renvoie à un fort investissement culturel et à une réévaluation de la profession. Au final, le processus de normalisation professionnelle auquel elle contribue favorise pour elle une forme de rédemption sociale.

\section{Déclassement et second choix}

Âgée de 37 ans, Madame Maceira est issue d'une famille d'universitaires: ses deux parents ont suivi des études de théologie avant d'exercer la profession de pasteur.

En suivant un apprentissage d'employée de commerce, Madame Maceira s'engage sur une voie professionnelle culturellement déclassée par rapport à celle de ses parents. Mais elle n'exercera pourtant jamais le métier d'employée de commerce, et toute la suite de son parcours professionnel peut être lue comme une tentative de renouer avec le pôle culturel de l'espace social, au travers de la profession infirmière qu'elle perçoit comme ouverte, évolutive et réflexive.

Alors qu'elle connaît une période de chômage à l'issue de son apprentissage, le souhait d'entreprendre une formation de bibliothécaire manifeste ainsi ses premières intentions de rapprochement avec une activité à connotation culturelle. Mais c'est à ce moment-là que s'offre à elle l'opportunité d'entrer en contact avec les soins: sa belle-mère, qui exerce comme soignante en milieu psychiatrique, lui propose de travailler avec elle comme aide-soignante durant quelque temps. Ce qui la mènera à abandonner son projet d'apprentissage de bibliothécaire:

"Je voulais commencer un apprentissage de bibliothécaire et puis en fait, finalement, je me suis dit: "Pourquoi pas l'école d'infirmière ?"» 
Elle se lance alors dans cette formation, en choisissant la filière de psychiatrie qui est encore proposée à l'époque ${ }^{106}$. Les propos de Madame Maceira («pourquoi pas») montrent que ce choix professionnel résulte clairement d'une opportunité très éloignée de ce que d'aucuns qualifieraient de "vocation». Car avant sa brève expérience en psychiatrie, elle n'avait jamais envisagé ce métier dont elle méprise même certains aspects pouvant être considérés comme les plus caricaturaux tels que le port de l'uniforme, le milieu hospitalier et le contact direct avec des émanations corporelles:

"J'ai jamais voulu être infirmière. Je savais pas ce que je voulais faire de ma vie, mais en tout cas pas infirmière. [...] Je voulais pas avoir une blouse, être dans un hôpital, faire des prises de sang. Enfin, je trouvais ça vraiment crétin comme métier!»

\section{"J'aime bien tout ce qui est de l'ordre de la culture"}

Près de vingt ans après avoir entrepris sa formation, et bien que la psychiatrie lui ait permis de se distancier de cette image repoussoir de l'infirmière hospitalière, elle tient à souligner que ses aspirations culturelles auraient très bien pu s'exprimer au travers d'une autre activité professionnelle:

"Je pense que si j'avais trouvé du travail [comme employée de commerce], peut-être que j'aurais souvent changé, pis je serais partie dans quelque chose de plus créatif, parce que j'aime bien tout ce qui est de l'ordre de la culture, de... artistique. Mais je pense pas qu'à un moment je me serais dit: "Oh, ça me manque la psychiatrie, je veux faire l'école!" Non, je pense pas, hein. Enfin, j'aurais même pas vu. Je pense que si j'avais trouvé du travail, je me serais pas retrouvée en psychiatrie. Donc j'aurais jamais su ce que c'était, donc ça m'aurait jamais intéressée.»

Et la manière dont elle s'efforce de modérer son enthousiasme lorsqu'elle recourt spontanément au terme de "passion » semble témoigner de la distance qu'elle entend maintenir avec une pratique qui, bien qu'elle lui permette d'actualiser ses dispositions,

\footnotetext{
106 Trois filières de formation étaient proposées jusqu'en 1993: soins généraux, psychiatrie, et hygiène maternelle et infantile.
} 
demeure toutefois inférieure au statut auquel elle aurait pu aspirer compte tenu de ses origines:

"Après, c'est devenu ma passion! Enfin, la psychiatrie, c'est ma passion, et puis les... Enfin, je trouve ça passionnant; je peux pas dire que c'est ma passion, j'ai d'autres passions dans la vie. Mais je trouve passionnant.»

\section{«Moi ce qui m'intéressait, c'était de soigner la tête, mais en tout cas pas le corps"}

La manipulation du corps des patients et de ses émanations représente clairement le «sale boulot» aux yeux de Madame Maceira, soit une activité dont elle tentera toujours de se distancier:

"Beaucoup d'infirmiers adorent ce côté-là: préparer un petit chariot, faire un pansement, regarder des plaies, faire des soins, poser une sonde, un drain, un cathéter. Mais alors moi, c'est le truc, pouh! Rien que la prise de sang, c'était déjà un cauchemar. J'ai jamais aimé ça.»

Ce rejet de la proximité corporelle lui fera même hésiter à entreprendre sa formation en soins. Car bien qu'elle ait choisi la filière de psychiatrie ${ }^{107}$, les deux premières années de formation font une large place aux soins somatiques. Contrainte de passer par là, elle s'arrangera tout de même pour déléguer à ses collègues les tâches qu'elle juge les plus rebutantes:

«ll y avait deux ans de soins physiques. Et je voulais pas faire l'école parce que je voulais pas faire ça, parce que ça m'intéressait pas. Mais je l'ai fait. [...] Il y avait que la dernière année qui était en psychiatrie. Donc j'ai fait deux ans de soins généraux, de chirurgie, de médecine, de ci, de ça. C'était vraiment pas mon truc. J'attendais impatiemment d'être en psychiatrie, et dès que j'étais en psychiatrie, dès qu'il y avait

\footnotetext{
${ }^{107}$ Précisons que, en comparaison avec celles d'autres secteurs, les infirmières de psychiatrie ne sont que rarement confrontées aux émanations corporelles des patients. À la question «à quelle fréquence êtes-vous personnellement occupée à nettoyer de l'urine et des selles", elles indiquent une fréquence faible et comparable à celle des infirmières scolaires, des infirmières d'entreprise, de cabinet médical et d'établissements socio-éducatifs. Elles se situent à l'opposé des infirmières exerçant dans des secteurs tels que les soins intensifs, la pédiatrie, les soins palliatifs et, surtout, la gériatrie.
} 
un soin, ne serait-ce qu'une prise de sang, [...] il y avait toujours des collègues qui venaient des soins généraux qui étaient enchantés de faire des soins, donc moi je leur laissais. Moi ce qui m'intéressait, c'était de soigner la tête, mais en tout cas pas le corps!»

\section{La psychiatrie, lieu d'apesanteur sociale}

Son diplôme de soins infirmiers en poche, et en cohérence avec ses aspirations, Madame Maceira évite ce lieu d'initiation que constituent les secteurs hospitaliers somatiques ${ }^{108}$ pour se diriger directement vers un secteur psychiatrique dans lequel elle exercera durant douze ans. Dans ce milieu où le travail sur le corps du patient est réduit à sa portion congrue, les statuts hiérarchiques sont euphémisés, non seulement par l'effacement des signes externes liés à l'uniforme ("C'est pour ça que je suis en psychiatrie: où j'ai travaillé, il y a pas de blouse»), mais aussi parce que les nombreuses formations continues offertes aux infirmières favorisent leur sentiment d'autonomie à l'égard des médecins:

"Le fait de travailler à [hôpital psychiatrique] était valorisé, du fait que l'infirmière avait un rôle très très autonome. [...]. On avait parfois des médecins psychiatres qui parlaient pas français, qui avaient aucune expérience de la psychiatrie, et où nous en tant qu'infirmiers, ben on soufflait un peu au médecin. [...] On leur apprenait les choses, donc on les formait. [...] On avait trop de responsabilités, mais c'est ça qui me plaisait aussi. Je pense que j'aurais pas pu travailler dans les soins si j'avais été dans un hôpital avec visite médicale le matin, on va au pied de chaque patient, euh: "Ah, ouais, celui-là vous lui donnerez un peu plus de ci, celui-là vous lui donnerez un peu plus ou moins de ça, merci au revoir!" Ça m'aurait pas plus. Là, c'était génial, parce qu'il y avait des groupes, parce qu'on travaillait avec les patients, parce qu'on se posait des questions, parce qu'on savait les traitements, parce qu'on les apprenait, parce qu'on avait de la formation continue, parce qu'on était formés en systémique, pour faire des évaluations de troubles anxieux, pour la thérapie cognitive, pour utiliser un tas d'outils, on était formés pour ça et on utilisait ça toute la journée.»

\footnotetext{
${ }^{108}$ Parmi les infirmières qui exercent en psychiatrie, 5,7\% ont déjà exercé aux soins intensifs, 4,8\% aux soins continus, 8,3\% aux urgences, 14,0\% en médecine et $16,6 \%$ en chirurgie.
} 
Madame Maceira manifeste alors une véritable soif pour les formations continues qui semblent lui offrir l'opportunité de renforcer son capital culturel et de vivre pleinement ce sentiment d'apesanteur sociale typiquement recherché par les déclassés sociaux. Durant ses douze années de travail en milieu psychiatrique, ces formations la mèneront d'abord à assurer l'encadrement de la formation continue de ses collègues, pour finalement la faire accéder au statut de praticienne formatrice qui lui permet d'encadrer les étudiantes et étudiants effectuant un stage en milieu psychiatrique.

\section{"Je n'avais plus tellement envie de m'occuper des gens"}

Ces nombreuses formations entraînent un double mouvement de Madame Maceira au sein de l'espace infirmier. Un mouvement vertical d'une part, qui la mène à s'éloigner toujours davantage du contact direct avec les patients pour se diriger davantage vers des tâches d'enseignement et d'encadrement; un mouvement horizontal d'autre part, qui contribue à brouiller son identité professionnelle pour aboutir à une sorte d'interchangeabilité des rôles avec les professionnels issus de la psychiatrie, de la psychologie et du travail social.

C'est ainsi qu'après douze années d'exercice en milieu psychiatrique, Madame Maceira souhaite un changement d'environnement: «Parce qu'en fait, je n'avais plus tellement envie de m'occuper des gens.» Elle intègre alors un centre ambulatoire de traitement des addictions. Si son intention est dès le départ d'abandonner les soins pour se consacrer exclusivement à la prévention, elle va cependant devoir partager son temps entre prévention et intervention psychosociale auprès des patients:

"Mon objectif en arrivant à [institution de traitement des addictions] c'était de tout de suite faire que de la prévention. [...] Donc ça a duré trois ans jusqu'à ce que ça se mette en place, mais c'était mon objectif quand même.»

Mais bien qu'elle doive maintenir une activité clinique, le passage d'un service de psychiatrie à un centre ambulatoire de traitement des addictions lui permet de se distancier encore davantage du corps des patients, notamment par l'abandon de toute distribution de médicaments ou de prises de sang: "La personne vient pour des entretiens, donc on prescrit pas, on n'a pas de pharmacie, on prescrit pas de traitement, on fait pas de prises de sang. » Et tout en prenant l'apparence d'une pratique libérale, son 
activité clinique se confond de plus en plus avec celle d'autres professionnels:

"On a nos patients et on les accueille, on fait des suivis, des entretiens, des prises en charge comme un psychologue ou un médecin psychiatre, mais à notre niveau d'infirmier.»

Car bien qu'elle parle d'une intervention se situant «à notre niveau d'infirmier», force est pourtant de constater que sa pratique semble largement superposable avec celle d'autres professionnels. Cette interchangeabilité des rôles se manifeste en particulier par les critères d'attribution de nouveaux patients, où la disponibilité, l'affinité personnelle ou encore le genre apparaissent comme plus déterminants que la spécificité professionnelle:

"-Qu'est-ce qui fait qu'un patient va être dirigé vers une infirmière plutôt que vers un éducateur, une assistante sociale, enfin, comment ça se passe?

- Ça, ça dépend souvent des disponibilités, ou alors ça peut dépendre aussi de la demande de la personne, selon l'histoire du parcours des gens. Peut-être même le médecin traitant nous appelle, fait la demande pour le patient, et pis là on peut voir si c'est peut-être mieux que ce soit un homme, ce serait peut-être mieux que ce soit une femme, ou ce serait mieux...

- C'est effectivement interchangeable alors, avec les éducateurs?

- Oui.»

Tout se passe en fait comme si les formations continues tendaient à prendre le pas sur la formation de base, pour petit à petit former le véritable socle commun entre ces professionnels: "Parce qu'on a tous la même formation, et puis on offre le même type de prise en charge.»

\section{"On est infirmière, et pis après on peut vraiment tout faire!"}

Après avoir partagé durant trois ans son temps entre prévention et intervention psychosociale, Madame Maceira abandonne totalement son travail auprès des patients: au moment où nous menons l'entretien, et alors qu'elle est âgée de 37 ans, elle vient d'intégrer un poste à responsabilité au sein d'une section de l'ASI.

En pleine cohérence avec sa trajectoire et sa position, Madame Maceira livre une définition de la profession dans laquelle le diplôme en 
soins apparaît comme une sorte de socle commun à partir duquel s'ouvre un espace infini des possibles:

"Le métier d'infirmier est passionnant parce que, justement, il est ouvert sur tout, ça veut dire que quand je vais à des formations ou bien à des journées thématiques dans les écoles, et que je vois des infirmières qui à la base étaient infirmières, qui maintenant suivent justement des masters... en sociologie, en ci, en ça, je trouve ça génial parce que c'est vraiment une base, voilà, on est infirmière, et pis après [...] on peut vraiment tout faire! [...] Être infirmière, c'est aussi participer au projet de santé en général, de la vie et de la société en général, vraiment de la santé avec un grand "S", [...] pas seulement sur le terrain, mais aussi au niveau politique, au niveau des formations, enfin c'est vraiment participer au projet santé général pour tout un chacun. C'est pas juste être au lit du patient et pis soigner la personne, c'est vraiment participer à tout ce qui va permettre à la personne d'être soignée. »

Dans une telle perspective, la profession infirmière ne peut se résumer à un ensemble de tâches, mais se définit au contraire comme une posture réflexive transversale à tous les domaines d'activité:

"Les soins infirmiers, c'est pas juste faire des piqûres pis juste prendre les ordres du médecin et puis faire ce que le médecin a dit. Il y a tout le fait d'être auprès du malade dans les hôpitaux et puis de se dire: "Tiens, ce matin il y avait ça, maintenant il est comme ça, hier c'était comme ça, tiens je lis son dossier, ah, oui, il a eu ci, il a eu ça". C'est pas le docteur House, mais y a tout ce schéma que l'infirmière se fait. [...] D'avoir toute cette démarche qui se fait dans la tête. Donc c'est très, très vaste. "

Selon Madame Maceira, cette posture réflexive est précisément ce qui distingue la profession infirmière des professions subalternes. Elle considère ainsi d'un œil critique l'arrivée massive d'assistantes en soins et santé communautaire (ASSC ${ }^{109}$ ) ou encore la menace

${ }^{109}$ Les infirmières issues de la fraction culturelle des classes moyennes sont celles qui se montrent les moins optimistes par rapport à l'arrivée des ASSC. Sur une échelle de 1 à 7 , elles présentent la moyenne la plus faible sur la proposition "L'arrivée des ASSC dans mon service/secteur d'activité me permet (ou me permettra) de consacrer davantage de temps à mon rôle professionnel». 
d'une implantation d'écoles de niveau ES en Suisse romande. Ces professions subalternes sont en effet susceptibles à ses yeux de remplacer les infirmières de niveau HES, alors que c'est justement la posture réflexive qui leur fait défaut. D'où un risque, selon elle, d'une baisse de la qualité des soins:

"Pour l'instant, la prise de position est plutôt de valoriser les HES et de ne pas entrer en matière par rapport aux formations ES, par la crainte d'avoir une formation à double vitesse avec ceux qui ont été refusés en HES qui vont en ES et puis de nouveau [...] d'avoir des infirmières à deux, trois, quatre niveaux. Parce que maintenant, il y a déjà les Assistantes en soins communautaires, enfin... II y a déjà plein de formations qui sont là et s'il y a encore les ES, ça fait encore une branche d'infirmières qui n'est pas... enfin, je peux pas dire des sous-infirmières, c'est pas ça, c'est pas juste. Mais avec le risque comme il y a déjà maintenant, dans les homes, vous avez une infirmière diplômée, et puis dix assistantes en soins communautaires. [...] Donc ça veut dire qu'il y a une perte au niveau quand même des connaissances, il y a une perte au niveau de la qualité du travail, et puis il y a une perte aussi au niveau de l'offre en soins par rapport par exemple au home qui va offrir dix assistantes en soins communautaires qui sont formées et qui sont bien formées, mais pour leur travail à elles. Sans qu'il y ait plus l'infirmière diplômée, l'infirmière HES qui, elle, a une autre formation, a d'autres compétences et qui est plus aussi par rapport à tout ce qui est de l'ordre de la réflexion, de la pensée, qui n'est pas seulement dans I'action. Et avec les ES, il y a le risque voilà, il y aura peut-être même plus d'infirmières diplômées, il y aura des ES, il y aura plus des HES, il y aura des ES et des assistantes en soins communautaires. Ce qui veut dire qu'il y a de nouveau une perte de la qualité au niveau des soins infirmiers, parce que les soins infirmiers c'est pas juste faire des toilettes, des prises de sang et donner à manger et distribuer des médicaments. C'est pas ça. Les soins infirmiers, c'est beaucoup plus vaste, c'est beaucoup plus complexe.»

Le cas de Madame Maceira éclaire l'effet de l'origine sociale sur un mode particulier d'appropriation de la profession infirmière: celui que nous avons qualifié de "désenchanté» et dont les analyses statistiques révèlent qu'il est avant tout le fait des infirmières issues de la fraction socioculturelle des classes moyennes. 
Souvent au bénéfice d'un fort capital culturel hérité, ces infirmières n'éprouvent pas d'attirance a priori pour la profession. Leur choix s'effectue tardivement, et apparaît comme une opportunité leur permettant de conformer leur poste à leurs (pré)dispositions. Une conformation qui s'exprime avant tout par une mise à distance du corps du patient et de ses émanations. Car non seulement le maniement des corps s'apparente sans doute à une activité trop «manuelle» relativement à leurs aspirations, mais il suscite de surcroît leur méfiance du fait qu'il implique des tâches qui, manifestement «médico-déléguées», renvoient à la subordination aux médecins.

Les formations continues représentent pour ces infirmières le plus sûr moyen de redéfinir leur profession non plus comme une série de tâches, mais comme un type de raisonnement pouvant se déployer dans tous les secteurs de l'activité humaine. Cette ouverture complète de la profession se manifeste d'abord par un rejet de tout repli disciplinaire: militant pour une forme d'interdisciplinarité, ces infirmières ne se montrent pas spécialement attachées à la constitution d'un corps de savoirs proprement infirmier. Dans cette perspective, l'interchangeabilité des rôles avec d'autres professionnels leur apparaît comme une manifestation de l'ouverture qu'elles revendiquent.

Ainsi affranchie de toute frontière, la profession infirmière leur permet d'éprouver un sentiment d'apesanteur sociale et de conformer leur activité quotidienne à leurs aspirations culturelles. Mais une telle définition de la profession comporte un risque, celui de laisser à d'autres catégories professionnelles le champ libre pour une définition plus circonscrite et manuelle de l'activité. Symboles d'une telle définition, les ASSC autant que les infirmières de niveau ES apparaissent ainsi comme une menace contre laquelle s'érigent ces professionnelles en défendant une formation de niveau académique. 


\section{Chapitre 12}

\section{"Technique et relationnel »: les dessous d'une doxa professionnelle}

La distinction - pour ne pas dire l'opposition - entre le «technique» et le «relationnel» constitue un élément incontournable de l'ethos infirmier. Omniprésente dans les discours professionnels, elle fonctionne comme principe de classement des actes autant que des personnes: il existerait ainsi des actes «techniques» et des actes «relationnels», ou encore des infirmières «techniciennes» et des infirmières «relationnelles» (VÉGA, 1997).

Selon Anne Paillet (2007), les dimensions «techniques» et «relationnelles» recouvrent un enjeu fondamental, celui d'une "humanisation» des soins qui représente une véritable "mission» pour la profession infirmière: "le risque de délivrer des soins déshumanisés, centrés sur la seule dimension technique, doit être en quelque sorte limité grâce à l'exercice des rôles complémentaires» (p. 193). La profession se retrouve ainsi confrontée à une situation paradoxale dans laquelle elle tend à minimiser la part la plus prestigieuse de son activité («technique») au profit de la dimension «relationnelle» qui, bien que s'inscrivant dans son projet d'humanisation, demeure toutefois faiblement valorisée. Et c'est ainsi que la dimension «relationnelle» en vient à remplir une véritable fonction de socle identitaire, ou encore de «ciment 
tempérant l'hétérogénéité manifeste de la profession" (РетітAт, 1994, p. 245).

Deux principes ont orienté la manière dont nous avons analysé nos données relatives aux dimensions "relationnelle" et «technique». Premièrement, il nous semble que ces deux dimensions ne peuvent être pensées séparément. Tout indique en effet que l'évocation de l'une se réfère nécessairement à l'autre, même implicitement. Nous allons même plus loin, en considérant que le couple «technique/relationnel» et le subtil équilibre dont se réclame chaque infirmière pour justifier de soins "humanisés» peuvent être considérés comme formant une doxa au sens de Pierre Bourdieu, soit l'une de ces "grandes oppositions obligées qui, paradoxalement, unissent ceux qu'elles opposent, puisqu'il faut avoir en commun de les admettre pour être en mesure de s'opposer à leur propos, ou par leur entremise, et de produire ainsi des prises de position immédiatement reconnues comme pertinentes et sensées par ceux-là mêmes à qui elles s'opposent et qui s'opposent à elles" (Bourdieu, 2003, p. 145).

Le deuxième principe qui a guidé nos analyses est la distance critique face au consensus apparent qui semble englober la définition de ces deux dimensions au sein de la profession. Nos analyses révèlent en effet que l'expression «relationnel» recouvre en fait des acceptions différentes selon les secteurs de l'espace infirmier.

\section{Humanisation des soins et valorisation de la dimension « relationnelle »}

Notre questionnaire contenait les deux propositions suivantes: "C'est la dimension relationnelle qui m'a motivée à devenir infirmière "; "C'est la dimension technique qui m'a motivée à devenir infirmière». La manière dont les infirmières 
Tableau 7. Composante relationnelle et technique de la motivation à devenir infirmière

\begin{tabular}{|l|c|c|c|}
\cline { 3 - 4 } \multicolumn{2}{l|}{} & $\begin{array}{c}\text { La dimension } \\
\text { relationnelle }\end{array}$ & $\begin{array}{c}\text { La dimension } \\
\text { technique }\end{array}$ \\
\hline $\begin{array}{l}\text { Ne s'applique pas } \\
\text { du tout/pas vraiment }\end{array}$ & $\%$ & 10,6 & 28,4 \\
\hline $\begin{array}{l}\text { S'applique } \\
\text { partiellement/tout à fait }\end{array}$ & $\%$ & 89,4 & 71,6 \\
\hline \multirow{2}{*}{ Total } & $\mathrm{n}$ & 2848 & 2810 \\
\cline { 2 - 4 } & $\%$ & 100 & 100 \\
\hline
\end{tabular}

Source : EPRIS.

Note: les deux propositions étaient «C'est la dimension relationnelle qui m'a motivée à devenir infirmière » et «C'est la dimension technique qui m’a motivée à devenir infirmière».

se positionnent face à ces deux propositions ${ }^{110}$ livre plusieurs enseignements relatifs à la doxa infirmière.

Relevons d'abord que ces propositions récoltent une très forte adhésion: les dimensions technique et relationnelle ont joué un rôle dans le choix professionnel pour respectivement sept et neuf infirmières sur dix (tableau 7). Ce qui souligne le rôle déterminant que jouent ces deux dimensions dans le rapport qu'entretiennent les infirmières avec leur profession ${ }^{111}$.

\footnotetext{
110 Pour chacune de ces propositions, les infirmières étaient invitées à se positionner sur une échelle à quatre modalités: "ne s'applique pas du tout", "ne s'applique pas vraiment", "s'applique partiellement» et "s'applique tout à fait». Dans les analyses qui suivent, ces quatre modalités ont été regroupées en deux modalités.

${ }^{111}$ Le questionnaire proposait une liste de douze motivations différentes pour devenir infirmière. Seule "Venir en aide aux personnes en souffrance" récolte une adhésion comparable à celle aux dimensions technique et relationnelle. Les neuf motivations restantes récoltent des adhésions bien moindres.
} 
Il faut ensuite s'intéresser à la relation qu'entretiennent les dimensions «relationnelle» et «technique» dans les réponses des infirmières. Alors qu'une représentation spontanée pourrait laisser entendre qu'une infirmière adhérant fortement à l'une devrait avoir tendance à rejeter l'autre ${ }^{112}$, les résultats montrent que ces deux dimensions sont au contraire fortement associées: plus une infirmière adhère à la dimension relationnelle et plus elle adhère aussi à la dimension technique et inversement ${ }^{113}$. Au niveau individuel, ces deux dimensions apparaissent donc comme cumulatives plutôt qu'exclusives.

Mais il ne faudrait toutefois pas en conclure que ces dimensions revêtent une égale importance aux yeux des infirmières: alors que plus de quatre infirmières sur dix affichent un profil "plus relationnel que technique», elles ne sont même pas une sur dix à afficher un profil inverse. Et si l'on s'intéresse aux infirmières qui présentent un profil «radical» (qui affichent une adhésion maximale à l'une des dimensions et un rejet maximal à l'autre), on s'aperçoit que 136 infirmières (soit près de $5 \%$ de l'échantillon) présentent un profil «radicalement relationnel», alors qu'elles ne sont que $12(0,4 \%)$, soit 10 fois moins, à présenter un profil «radicalement technique». Conformément à la doxa professionnelle, le profil infirmier modal est donc celui qui associe une très forte dimension relationnelle à une dimension technique égale ou moindre (voire absente). Le profil qui présente une tendance inverse est rare et, en son sein, l'absence de dimension relationnelle n'est qu'anecdotique ${ }^{114}$.

112 Une telle exclusivité entre les dimensions «relationnelle» et "technique» est non seulement suggérée par le langage professionnel commun, mais aussi par les classifications opérées par certains sociologues. En montrant que les infirmières évoluent dans un espace social compris comme un continuum, nos analyses présentées au chapitre 3 évitent un tel écueil: à l'échelle individuelle, chaque infirmière dispose à la fois de capital infirmier et de capital médical, certaines étant même également dotées de ces deux ressources (elles occupent alors une position située à équidistance des pôles infirmier et médical).

${ }^{113}$ Le coefficient de corrélation de Pearson qui mesure la force de la relation indique une relation positive et significative $(\mathrm{r}=0.35, \mathrm{p}<0.001)$.

${ }^{114}$ Relevons que l'adhésion à toutes les propositions du questionnaire relatives à la dimension "relationnelle» des soins est d'autant plus forte que 
Ces résultats nous permettent de préciser ce que recouvre le projet d' "humanisation» de la profession mentionné par Anne Paillet: tout se passe en fait comme si ce projet supposait une association spécifique entre les dimensions "relationnelle» et «technique». Alors que la première semble pouvoir se suffire à elle-même pour fonder une identité professionnelle, la seconde semble au contraire ne pouvoir tirer sa légitimité que de son association «minoritaire» à la première.

\section{Le couple "relationnel/technique » comme principe de classement et de déclassement : un dénigrement en cascade}

L'analyse des entretiens révèle toute la force de cette doxa qui fonctionne comme principe de classement et de déclassement. Nous n'avons en effet rencontré aucune infirmière revendiquant ouvertement le statut de "technicienne». Au contraire, chacune mentionne la forte composante "relationnelle» de sa pratique, y compris celles qui exercent dans les secteurs les plus médicalisés. Et l'on retrouve ici ce processus où l'idéal relationnel devient un moyen "de se dénigrer entre sous-groupes infirmiers" (VÉGA, 1997, p. 114). Plus précisément, on assiste à une sorte de «dénigrement en cascade», dans lequel chaque infirmière s'estime être "très relationnelle» ou, pour tout le moins, "plus relationnelle que d'autres» jugées «trop techniciennes». Exerçant en médecine, Madame Dunod décrit ainsi les infirmières de soins intensifs

la formation de la mère est faible, et ce indépendamment de la formation du père, de l'origine sociale ou de l'appartenance à l'une des quatre fractions. Il semble donc que l'adhésion à la dimension «relationnelle» soit fortement liée à une transmission de mère en fille: moins une mère est dotée sur le plan culturel et plus est grande la probabilité qu'elle encourage sa descendance à embrasser la profession infirmière tout en insistant sur la dimension relationnelle du soin. 
comme éloignées du véritable «rôle» infirmier en raison de leur profil trop «technique»:

"Eux [les soins intensifs], ils sont complètement axés sur le technique et ils valident pas du tout tout ce qui est notre rôle, [...] parce que pour eux le technique c'est beaucoup plus important que [...] tout ce qui est réseau, de tout ce qui est de globalité du patient.»

Pourtant, et pour peu que l'on donne la parole aux infirmières de soins intensifs, on s'aperçoit que ces dernières ne revendiquent aucunement un tel profil de pures techniciennes, insistant au contraire autant que les autres sur l'importance de la dimension relationnelle. Les propos de Madame Jacques semblent ainsi faire directement écho à ceux de Madame Dunod:

"On dit qu'aux soins intensifs il n'y a pas de relationnel, mais s'il n'y a pas de relationnel là, c'est horrible, parce qu'ils [les patients] traversent des crises, des moments de stress. Donc je me dis qu'il faut savoir être aidant et des fois... en un temps court en fait. Moi j'aime ça!»

Et tout comme Madame Dunod, elle mentionne un secteur dans lequel le relationnel n'est pas assez présent à ses yeux: "Par exemple, je savais que le bloc opératoire, voir les patients tout endormis, ça m'intéressait pas."

Le bloc opératoire, c'est précisément le secteur dans lequel exerce Monsieur Bideau en tant qu'infirmier anesthésiste. Loin d'estimer qu'il n'a affaire qu'à des patients "tout endormis», il revendique également la part «relationnelle» de son activité. Une relation dont l'intensité ne semble avoir d'égale que sa brièveté:

"Même si c'est une intervention en anesthésie locorégionale, l'infirmier anesthésiste ou le médecin anesthésiste est toujours là. C'est pas parce que le patient est conscient qu'on est pas là, on est toujours là. Donc on va causer avec, voir comment il va, essayer de le distraire aussi [...]. On a aussi cette part de relation qui 
doit être... qui est très intense, qui n'est pas très longue, qui est souvent très directe: "Au revoir", et toc! le patient il dort."

Le bloc opératoire se situant probablement en «bout de chaîne relationnelle», Monsieur Bideau ne mentionne pas de secteur qui lui semble moins «relationnel» que le sien. Ce qui ne l'empêche pas toutefois de mentionner une activité - le prélèvement d'organes sur des patients décédés - qui, en raison de son absence totale de dimension relationnelle, constitue un cas limite:

"Il y a aussi des interventions qui sont un peu plus délicates. Par exemple, quand on a des patients qu'on doit prendre en charge pour des dons d'organe. Ça, j'aime moins [...]. C'est très technique, ça, j'aime moins."

S’il ne correspond pas nécessairement à une opposition objective, le couple «relationnel/technique» constitue donc bel et bien une doxa professionnelle qui, inscrite au plus profond des consciences, agit comme schème de perception et comme principe de classement, de déclassement, voire de disqualification: il existe toujours des infirmières "moins relationnelles que soi», et celles qui sont décrites comme abandonnant cette dimension au profit de la seule dimension "technique» semblent faire office d'image repoussoir de la profession.

La dimension «relationnelle» apparaît ainsi à la fois comme un socle identitaire favorisant, au moins chez les nouvelles venues, un rapport enchanté à la profession ${ }^{115}$, et comme principe de classement. Ce qui suppose un certain consensus quant à ce qui fait ou défait une "véritable» infirmière.

Si un tel consensus semble effectif en ce qui concerne la définition de la dimension "technique» des soins (elle se réfère à l'usage d'appareils ou d'instruments venant médiatiser le contact entre l'infirmière et le corps du patient), il en va

${ }^{115}$ Sur le désenchantement que vivent souvent les étudiantes et étudiants en soins infirmiers relativement à cette dimension, voir THOuvenin (2004). 
différemment en ce qui concerne la dimension "relationnelle». Tout se passe ici comme si l'on avait affaire à un effet d'allodoxia, "quiproquo qui porte à prendre une chose pour une autre, en toute bonne foi" (Bourdieu, 1984a, p. 277). Le terme de «relationnel» prend en effet deux acceptions distinctes qui, jamais explicitées, semblent étroitement liées à la position occupée dans l'espace infirmier.

\section{Relationnel expressif et relationnel instrumental}

Caractéristique des infirmières du pôle infirmier de l'espace, la première acception est celle que nous qualifions de «relationnel expressif». Faisant écho à une conception humaniste du care largement véhiculée dans les représentations du rôle de l'infirmière, le "relationnel expressif» fait apparaître la relation comme une fin en soi, l'attention au corps devenant alors le support de cette relation:

"Je fais volontiers des massages du dos, pieds, épaules. [...] Parce que ça permet de rentrer dans une relation différente, dans la relation au corps, [...] c'est un autre chemin pour rentrer avec les gens. Des fois, on va prendre une pression chez quelqu'un, [...] et en prenant la pression et en discutant avec la personne, on la connaît sous un angle. Et puis un jour, on est amenée à faire la douche de cette personne et on la connaît sous un autre angle. Ça permet de compléter un peu la relation qu'on a.» Mme Maystre, soins à domicile

Dans le cadre d'un tel "relationnel expressif», la relation établie avec le patient est sous-tendue par un schème psychologique, qui peut être considéré comme une version sécularisée du schème religieux qui prévalait jusqu'au milieu $\mathrm{du} \mathrm{xx}^{\mathrm{e}}$ siècle parmi une certaine fraction des infirmières. Joëlle Droux (2000) rappelle en effet que, dans la perspective de la charité chrétienne, les soins du corps ne représentaient pas 
une fin en soi, mais bien un "préalable nécessaire» pour accéder à l'âme des personnes secourues: "Vous ne pouvez arriver à l'âme de vos pauvres qu'en soignant leur corps", rappelle ainsi un texte daté de 1888 et servant de base à l'enseignement des novices des Filles de la Charité à Fribourg (p. 93). À cette mission d'évangélisation qui, dans une perspective de contrôle social, charrie un ensemble de stratégies visant à faire accepter aux pauvres leur condition, le relationnel «expressif» substitue une mission de conversion des schèmes de perception corporels des personnes souffrantes:

"Même quelqu'un qui est malade, même qui a une leucémie, la personne âgée, ben elle peut être en santé quelque part dans son sentimental, et bien vivre avec ça quelque part.» Mme Dunod, médecine

"Je dirais que [l'infirmière] est un agent du mieux-être. Pour moi, cela doit être un agent de changement. Un agent qui fait que la personne va changer. [...] Donc une bonne infirmière va faire que l'autre change." Mme Emery, psychiatrie

Le psychisme succède à l'âme, la maladie chronique à la pauvreté chronique, mais l'essentiel demeure: les infirmières sont des "agents du mieux-être» qui permettent aux patients de "bien vivre avec ça», par-delà leurs souffrances physiques ou matérielles.

À ce «relationnel expressif» répond, du côté du pôle médical, un «relationnel» que l'on peut qualifier d'«instrumental». On assiste ici à un renversement des priorités: la relation avec le patient ne constitue plus une fin en soi, mais plutôt un moyen de rendre possible la prise en charge médicale. Nous avons vu plus haut que Madame Jacques, qui exerce aux soins intensifs, accorde une grande importance au "relationnel». La suite de son propos permet de constater qu'elle considère avant tout cette dimension des soins comme un acte de réorientation des patients agités, acte 
rassurant qui permet d'éviter d'avoir à «sauter sur la seringue» pour les endormir:

"On dit qu'aux soins intensifs il n'y a pas de relationnel. Mais s'il n'y a pas de relationnel là, c'est horrible! [...]. Par exemple, les accidentés de la route, les polytraumatisés ou les traumatismes crâniens, [...] c'est important à ces phases-là d'essayer de communiquer avec eux [...] Parce que c'est un peu comme un trou noir, ils ne savent plus ce qui s'est passé. Donc de les resituer; de dire où ils sont, de redire la date, de dire ce qui leur est arrivé [...] Ça aide à ce qu'ils se calment, une fois qu'ils ont compris où ils sont, qu'est-ce qui leur est arrivé, qu'ils sont pris en charge, que c'est normal qu'ils ne peuvent pas parler avec le tube, et ben il y a des fois, ça les rassure, et puis on est pas obligés de sauter sur la seringue."

Loin de viser une parole cathartique censée «libérer» la charge émotionnelle du patient, le "relationnel instrumental» consiste plutôt en un échange d'informations entre les professionnels et les patients. Et la fonction de cet échange semble bien de favoriser une certaine compliance médicale, comme l'explicite le propos de Monsieur Ropars (urgences), qui restitue au terme de "patient" sa double acception, nom propre et adjectif:

"Si on explique aux patients ce qui se passe, ils sont calmes, ils attendent, ils comprennent et ils remercient. [...] Moi j'utilise le relationnel [...] S'ils sont en confiance, on a tout gagné. Il va être patient."

Derrière l'apparent consensus que semble recouvrir le recours spontané et permanent au «relationnel»se cachent ainsi des pratiques qui révèlent deux acceptions différentes de cette notion. Si notre enquête laisse supposer que la distinction entre le «relationnel expressif» et le "relationnel instrumental» recouvre celle entre le "pôle infirmier» et le "pôle médical» de l'espace, il n'en demeure pas moins que chaque infirmière est 
susceptible de recourir à ces deux acceptions, en fonction des situations de soins qui se présentent à elle.

En tant que doxa professionnelle, le couple «techniquel relationnel» peut sans doute être considéré comme le socle identitaire de la profession infirmière. Qu'elles exercent au pôle médical ou au pôle infirmier de l'espace, l'immense majorité des infirmières se réclame en effet d'une pratique composée d'une forte composante relationnelle mâtinée d'une composante technique. Mais derrière cet apparent consensus se dissimule une lutte de légitimité, chaque infirmière ayant tendance à dénigrer des collègues dont la pratique «manque de relationnel» à ses yeux. Si une telle vision professionnelle sous-entend l'existence d'un continuum allant du «moins relationnel» au "plus relationnel», l'examen des discours et pratiques conclut à l'impossibilité d'un tel continuum, en raison d'acceptions distinctes du terme «relationnel». 



\section{Conclusion}

Alors que la profession infirmière jouit d'une forte visibilité dans l'espace public et qu'elle est fortement implantée dans l'imaginaire collectif (qui n'a jamais eu affaire à une infirmière?), elle demeure paradoxalement très mal connue. Du côté des services publics, il n'existe par exemple aucun registre statistique en Suisse permettant de recenser le nombre d'infirmières en exercice ni leur répartition dans les différents secteurs de soins. Du côté de la recherche, on alterne généralement entre d'une part des analyses très localisées et le plus souvent hospitalocentrées, et d'autre part des analyses très générales décrivant la profession comme un ensemble homogène.

L'objectif de notre recherche était de dépasser cette alternative analytique, en s'attachant à livrer une véritable "photographie" de la profession, de saisir ce qui fait son unité mais aussi sa diversité, à une échelle globale autant que locale. Notre point de départ est le constat d'un double processus de différenciation de la profession au cours des dernières décennies. Une différenciation horizontale d'abord, avec la multiplication des secteurs extrahospitaliers faisant suite à l'augmentation des maladies chroniques et aux politiques de "rationalisation» des coûts de la santé. Une différenciation verticale ensuite, avec l'académisation de la formation ainsi que l'institutionnalisation 
d'une hiérarchie propre à la profession. Comme nous l'avons montré, ce double mouvement de différenciation entraine un renforcement de la lutte pour la définition légitime de la pratique soignante, lutte qui distingue plusieurs fractions d'infirmières au sein d'un espace professionnel diversifié, inégalitaire et dynamique.

\section{Un espace infirmier diversifié, inégalitaire et dynamique}

L'espace infirmier que nous avons proposé est structuré selon deux dimensions. Une dimension horizontale d'abord, qui distingue les infirmières selon la structure de leur capital, c'està-dire selon les proportions respectives des capitaux infirmier et médical en leur possession. Cette dimension tend à distinguer les infirmières exerçant dans les secteurs hospitaliers de celles exerçant dans les secteurs extrahospitaliers. Une dimension verticale ensuite, qui distingue les infirmières selon le volume plus ou moins important de leur capital, et qui les distribue sur une échelle hiérarchique, cette hiérarchie étant matérialisée par le statut professionnel et par le prestige associé au secteur d'activité.

Quatre fractions d'infirmières composent cet espace: les dominantes médicales, les dominées médicales, les hétérodoxes et les élites infirmières. Trois de ces fractions se caractérisent par des modalités spécifiques de la lutte pour une définition légitime des soins. Les dominantes médicales d'abord, qui incarnent une stratégie que l'on pourrait qualifier de "conservatisme pragmatique». Exerçant dans un contexte de soins aigus qui rend nécessaire une certaine indifférenciation des rôles (Liberati, 2017), l'infirmière se présente ici comme le «bras droit" du médecin. Bien qu'étant peu autonome, elle retire un certain prestige de cette proximité physique et symbolique entretenue avec le corps médical. Si elle peut à l'occasion 
avancer des revendications, ces dernières portent davantage sur les conditions du travail que sur son contenu: il s'agit avant tout de lutter pour l'obtention d'une meilleure place au sein de la hiérarchie médicale. Les élites infirmières ensuite, qui tentent de mettre en œuvre une autonomie de type «scientificomanagériale» en développant un capital infirmier composé de savoirs scientifiques produits par et pour les infirmières d'une part, de connaissances managériales d'autre part. L'infirmière se présente ici non seulement comme celle qui produit les savoirs favorisant l'amélioration des pratiques, mais aussi comme celle qui assure la coordination entre les multiples professionnels qui gravitent autour des patients. Investissant les terrains scientifique et managérial, elle délaisse quelque peu le terrain de la prise en charge clinique, échappant ainsi à la mise en concurrence directe avec les médecins. Les hétérodoxes enfin, qui luttent pour une autonomie du même nom en s'appuyant sur un capital infirmier composé de savoirs paramédicaux d'une part, de sciences humaines et sociales d'autre part. L'infirmière propose ici une prise en charge alternative du patient, et remet ainsi en question la hiérarchie médicale par l'instauration d'une logique spécifiquement infirmière à l'aune de laquelle serait référé le contenu de son activité ${ }^{116}$. Ce faisant, elle court le risque de se retrouver en porte-à-faux tant avec les médecins qu'avec les plus orthodoxes de ses collègues infirmières, risque qu'elle atténue par une mise en œuvre plus ou moins clandestine de certaines de ses pratiques et par un exil vers la région la moins médicalisée de l'espace.

\footnotetext{
${ }^{116}$ Les hétérodoxes sont sans doute les infirmières les mieux prédisposées à la contestation, cette dernière pouvant prendre des formes plus radicales que la «mobilisation consensuelle» typique de la profession décrite par Ivan Sainsaulieu (2012). Mais elles bénéficient en même temps de conditions matérielles relativement avantageuses, notamment en raison d'un revenu plus élevé du conjoint qui leur permet de réduire leur taux d'activité (voir chapitre 9). Ainsi s'explique, peut-être, que leurs prédispositions subversives ne rencontrent que partiellement les conditions de leurs mises en œuvre.
} 
Les trois modalités de lutte que nous avons mises en évidence recouvrent en partie les trois dimensions constitutives du travail infirmier identifiées par certains auteurs: spécialisation et technique, coordination et interprofessionnalité, polyvalence et relation. Mais en montrant que ces représentations et pratiques sont incarnées par des fractions qui occupent des positions distinctes au sein de l'espace infirmier, nos résultats viennent prolonger et compléter ceux qui, issus d'approches plus microsociologiques, ont mis en évidence le "malaise structurel» que ces représentations et pratiquent entraînent à l'échelle individuelle (DuBET, 2002), ou encore les tensions interindividuelles qu'elles suscitent à l'échelle des collectifs de travail (ACKer, 1995; Feroni \& Kober, 1995; VÉGA, 1997). Notre approche nous a ainsi permis de dépasser les perspectives parcellaires autant que les visions par trop homogénéisantes, et d'identifier les éléments qui à la fois distinguent et unissent les membres de la profession infirmière.

Au-delà des différences, l'espace infirmier recouvre aussi des inégalités qui se manifestent par les rendements très inégaux des formes de capital. Nos analyses nous ont permis de remonter le fil de ces inégalités, en montrant que la hiérarchisation des secteurs suivant leur prestige s'explique directement par leur degré de médicalisation. Fortement intériorisées, les oppositions reposant sur les critères médicaux qui structurent les rapports sociaux au sein de la profession infirmière "tendent [ainsi] à s'imposer comme les principes fondamentaux des pratiques et de la perception des pratiques» (Bourdieu, 1979a, p. 191).

Cette perméabilité à des enjeux exogènes nous rappelle qu'aucun des deux types de capital identifiés n'est spécifique à l'espace infirmier. Pour ce qui est du capital médical, il est issu du champ qui domine et englobe l'espace infirmier. S'agissant du capital infirmier, nous avons vu qu'il est largement fondé sur une compétence relationnelle, compétence qui «repose sur un savoir pratique ordinaire [qui] n'est pas établi comme savoir ésotérique professionnel» (MILBuRn, 2002, p. 70). Plus 
précisément, la modalité "hétérodoxe» de ce capital ne peut être considérée comme spécifique à la profession, puisque largement fondée sur des savoirs et savoir-faire issus des sciences humaines et sociales. Quant à sa modalité «scientificomanagériale", les élites infirmières produisent certes un corps de savoirs reconnu comme appartenant en propre à la profession. Mais force est pourtant de constater que ce processus demeure embryonnaire, notamment parce que les sciences infirmières ne se sont implantées que récemment en Europe continentale - et en particulier en Suisse romande. Au total, il résulte de cette trop faible autonomie que la profession infirmière n'est pas à considérer comme un champ, mais bien plutôt comme un espace social inséré dans le champ médical dont il reproduit certains principes de classement.

Loin d'être figé, l'espace infirmier possède une dynamique propre, animée par l'importante mobilité professionnelle des infirmières. Alors que l'hôpital apparaît comme un "passage obligé» permettant l'accumulation de capital médical, l'avancée en âge entraîne une migration massive vers les secteurs extrahospitaliers, migration qui s'accompagne souvent d'une baisse importante du taux d'activité.

\section{Généralisation des résultats et enjeux pour l'avenir de la profession}

Dans quelle mesure nos résultats sont-ils généralisables? L'espace infirmier étant inscrit dans un champ médical dont on sait par ailleurs qu'il est fortement soumis aux standards internationaux (VASSY, 1999), on est en droit de s'attendre à retrouver des tendances similaires dans la plupart des pays occidentaux. Il est cependant probable qu'une comparaison internationale révélerait certaines variations relatives aux rapports de force entre les différentes fractions, en lien avec le processus d'académisation de la formation. On sait en effet que ce 
processus, relativement récent en Suisse romande, est déjà très avancé dans les pays anglo-saxons et en Europe du Nord. Or l'introduction de ces nouvelles formations académiques est susceptible d'influencer non seulement l'opposition primaire entre les infirmières du pôle médical et celles du pôle infirmier (soit l'opposition entre «conservatisme pragmatique» et "autonomisme») mais aussi, et peut-être surtout, l'opposition secondaire entre les élites infirmières et les hétérodoxes (soit celle entre l'autonomie "scientifico-managériale» et l'autonomie «hétérodoxe»). Comme l'illustre le cas particulier des infirmières titulaires du master, tout porte en effet à croire que l'académisation de la formation bénéficie prioritairement aux élites infirmières par un renforcement identitaire.

Ces considérations nous mènent à nous interroger sur l'évolution future de l'espace infirmier. Le double processus de différenciation est appelé à se poursuivre: alors que les politiques de raccourcissement des séjours hospitaliers continueront à renforcer la différenciation horizontale en augmentant le poids relatif des secteurs extrahospitaliers, l'académisation des formations contribuera à accentuer la différenciation verticale, par la hiérarchie qu'elle institue entre les infirmières respectivement titulaires d'un bachelor, d'un master et d'un doctorat. Et c'est donc sans doute au renforcement de la fraction des élites infirmières que conduira ce double processus. Alors que ces professionnelles n'avaient jusqu'ici accès qu'à des formations très hétérogènes en Suisse romande, l'ouverture de filières master et doctorat leur donne à présent la possibilité de suivre une formation académique homogène et spécifique à leur profession.

Si elle contribuera sans doute à la reconnaissance et à l'autonomie professionnelle, l'expansion de la fraction des élites infirmières doit aussi être considérée avec un recul critique. De fait, et comme nous l'avons montré, cette fraction qui cherche à développer la coordination et l'interprofessionnalité entretient une relation ambivalente avec le management, ce qui la rapproche de la fraction "réformatrice» identifiée par 
Patrice Pinell (2008) au sein de la profession médicale. Et l'on est en droit de se demander si, telles les infirmières laïques de la fin du XIX ${ }^{\mathrm{e}}$ siècle qui, voulant échapper au pouvoir ecclésiastique, furent récupérées par le pouvoir médical (SENOTIER, 1992), les élites infirmières contemporaines ne courent pas le risque d'être récupérées par le pouvoir managérial en voulant échapper au pouvoir médical ${ }^{117}$.

Afin de se prémunir contre un tel risque, il convient à notre sens d'éviter que les élites infirmières ne se coupent des trois autres fractions de la profession. Car une telle perte de contact pourrait avoir comme conséquence directe une dévaluation de l'activité clinique, activité qui se verrait alors davantage déléguée aux professions subalternes. En privant la profession infirmière de la "position unique» que lui procure sa proximité avec les patients (Svensson, 1996), cette délégation serait susceptible de l'affaiblir. Mais une déconnexion entre les élites infirmières et les hétérodoxes aurait aussi pour conséquence la dévaluation de la culture critique qu'incarnent ces dernières. Marginale au sein des sciences infirmières (Browne, 2001; ElziNGA, 1990), une telle culture critique demeure pourtant indispensable à l'identification et à l'analyse des rapports de pouvoir et donc à une meilleure prévention de toute nouvelle mise sous tutelle.

Le rapport que les élites infirmières entretiennent avec l'activité clinique représente donc un enjeu majeur pour le futur de la profession. Dans cette perspective, une sous-fraction devra être observée avec attention: celle des infirmières titulaires d'un master en sciences infirmières. Bien que peu nombreuses pour l'instant, ces infirmières pourraient cristalliser les enjeux entourant la pratique clinique. De fait, le rôle de "pratique infirmière avancée» auquel elles sont destinées semble intégrer pleinement cette activité, notamment par la mise en avant du

${ }^{117}$ Pour un développement sur ces questions, voir Toffel \& TAwfik (à paraître). 
soin direct aux patients en situation complexe. Ce nouveau rôle infirmier pourrait de ce fait contribuer à limiter la déconnexion entre les élites et les autres fractions.

Nous l'avons dit, le renforcement des élites infirmières aura une influence directe sur les hétérodoxes, dont le capital infirmier fortement teinté de sciences humaines et sociales pourrait subir une dévaluation relative. Il est en effet probable qu'une partie des infirmières qui auraient autrefois opté pour ce genre de formation se dirigent à présent vers les nouvelles filières de master et de doctorat en sciences infirmières. Cette dévaluation relative vient se conjuguer à un autre phénomène mis en évidence par nos analyses: celui du "double cloisonnement" des secteurs extrahospitaliers. Nous avons vu en effet que ces secteurs se trouvent relativement isolés vis-à-vis de la formation de base d'une part - rares sont les infirmières qui débutent directement leur carrière professionnelle dans ces secteurs -, vis-à-vis des secteurs les plus médicalisés d'autre part - plus rares encore sont celles qui quittent ces secteurs pour rallier un secteur extrahospitalier. Associé à la perspective de dévaluation relative, ce "double cloisonnement" est d'autant plus préoccupant que les secteurs extrahospitaliers constituent une région stratégique de l'espace infirmier, dans la mesure où ils sont destinés à une forte croissance de leurs effectifs. La valorisation du capital infirmier spécifiquement associé à ces secteurs - par exemple par une intégration plus marquée qu'elle ne l'est actuellement des savoirs issus des sciences humaines et sociales dans les formations de bachelor, master et doctorat - pourrait contribuer au décloisonnement de ces secteurs.

Une attention particulière devrait également être accordée aux dominées médicales, soit aux infirmières qui exercent majoritairement dans les secteurs hospitaliers semi-aigus. À la «misère de condition» qui caractérise cette région de l'espace (repérable notamment à l'intensité des soins de confort et d'hygiène synonyme de pénibilité physique) s'ajoute parfois 
ici une «misère de position" liée à l'abaissement relatif que ces infirmières peuvent éprouver au contact direct de leurs collègues dominantes médicales qui exercent dans les secteurs les plus prestigieux. En accentuant les inégalités, le processus de différenciation vertical qui touche la profession pourrait venir renforcer ce sentiment d'abaissement, ces infirmières risquant d'intérioriser le statut d' «infirmières de base» dont elles se voient parfois affublées par certaines de leurs collègues. Les politiques de formation ont sans doute leur rôle à jouer pour limiter un tel sentiment de déclassement, notamment par le refus d'un système de formation à deux vitesses et par la défense de l'accès de toutes les infirmières à une formation de base de niveau académique.

\section{Tout ça pour ça ? De quelques réflexions sur la réception de ce livre}

Penser la profession infirmière comme un espace social permet d'appréhender certains de ses enjeux fondamentaux. Reste à savoir comment ces résultats peuvent être perçus et transférés au sein des milieux professionnels, qu'il s'agisse de l'enseignement ou de la pratique.

La réponse à cette question suppose de tenir compte des inévitables difficultés de réception que peuvent susciter les résultats des recherches en sciences sociales auprès des personnes étudiées, en particulier lorsque ces recherches se réclament d'une posture critique. Parmi ces difficultés, on relèvera d'abord le sentiment d'évidence qui peut accompagner la découverte des résultats: "Était-il nécessaire de mener une telle enquête pour aboutir à des résultats qu'un peu de bon sens aurait suffi à révéler?" "L'espace infirmier, les quatre fractions qui le composent et l'échelle de prestige des secteurs ne relèvent-ils pas de l'évidence?" Classique de la réception des travaux sociologiques, ce sentiment d'évidence n'est en fait bien souvent 
qu'une illusion d'évidence. Car ces résultats ne semblent évidents qu'une fois établis. En témoigne le fait que, lors de la phase de préparation de notre recherche, nous n'avons trouvé aucune étude ni aucune personne capable de nous livrer une image d'ensemble de la profession ${ }^{118}$. Si nos résultats peuvent sans doute susciter à l'occasion un sentiment de réminiscence du fait qu'ils rendent explicite ce qui affleure parfois à la conscience sous forme d'intuition, ils ne font pas pour autant double emploi avec le sens commun, ne serait-ce que parce qu'ils mettent au jour les principes organisateurs d'une réalité que le sens commun ne fait qu'effleurer.

Les résultats de la recherche sociologique peuvent aussi susciter à l'occasion un sentiment de rejet. Toute démarche de recherche suppose en effet un parti pris, un point de vue sur la réalité qui, n'étant pas nécessairement superposable avec l'expérience subjective des personnes étudiées, peut avoir quelque chose de déstabilisant. Le fait même de se trouver "objectivé» par les instruments de la recherche - en particulier lorsqu'il s'agit d'instruments quantitatifs - comporte toujours une forme de violence liée à la réduction de la réalité que suppose tout travail d'objectivation: "même la description du plus petit fragment de la réalité ne peut jamais être pensée de manière exhaustive", disait Max Weber (1992, p. 156, souligné par l'auteur). Défini par l'infinité des caractéristiques qui forment sa singularité, l'«individu empirique» se trouve ici réduit à un "individu épistémique» incarné par les seules propriétés retenues pour l'analyse (Bourdieu, 1984a). Le sentiment de décalage que peut susciter une telle réduction se voit encore redoublé lorsque le sociologue se donne pour tâche de construire des typologies - ici

${ }^{118}$ À l'entame de notre projet, nous souhaitions rencontrer une infirmière porteuse d'une vision d'ensemble des différents secteurs de la profession et qui aurait pu intégrer notre équipe de recherche. Suite à de nombreuses entrevues, nous sommes finalement arrivés au constat qu'une telle personne n'existait probablement pas. Si ce constat nous a déçus, il nous a aussi définitivement convaincus de la pertinence de notre recherche. 
des fractions d'infirmières - qui peuvent être perçues comme

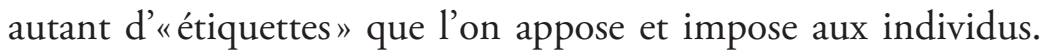
Et lorsque, non content de décrire des différences, le sociologue met à profit sa typologie pour révéler des inégalités symbolisées par des appellations explicites (dominantes médicales, dominées médicales, élites infirmières), l'incompréhension peut alors atteindre son paroxysme.

Par-delà les malentendus qui peuvent entourer la démarche sociologique, la méfiance à l'égard de certains résultats de recherche peut aussi s'enraciner dans des considérations d'ordre politique. Les infirmières qui se donnent pour projet d'émanciper leur profession ont en effet tout intérêt à la présenter comme une réalité indivisible, tout entière tournée vers l'émancipation. Dans cette perspective, la présentation d'un espace infirmier hétérogène et inégalitaire peut sembler contre-productive. Pire, la mise en évidence de prises de position étrangères à ce projet émancipatoire - comme on a pu l'observer en particulier parmi les dominantes médicales - peut être perçue comme une forme de contestation des arguments en faveur d'une émancipation ${ }^{119}$.

Afin de prévenir ces difficultés de réception classiquement rattachées à la sociologie, il convient d'abord de préciser que notre représentation de l'espace infirmier n'exclut nullement les nuances liées à des positions intermédiaires. Comme le suggère l'homogénéité du nuage de points figurant sur les graphiques 3 et 5 présentés dans cet ouvrage (chaque point représentant un individu), les différentes fractions ne constituent pas des blocs homogènes dans lesquels seraient enfermés les individus, mais

\footnotetext{
${ }^{119}$ C'est sans doute parmi la fraction des élites infirmières, avant-garde d'une autonomie scientifico-managériale, que la probabilité d'une telle méfiance à l'égard de nos résultats est la plus élevée. Et ce n'est peut-être pas le moindre des paradoxes que la fraction dont le développement contribue le plus à la diversification de la profession soit précisément celle qui trouve intérêt à la présenter comme un ensemble homogène.
} 
incluent au contraire un continuum de positions et de prises de position. En second lieu, cette représentation de l'espace infirmier ne propose jamais qu'une sorte de paire de lunettes qui, bien qu'offrant une vision nécessairement sélective, permet toutefois de révéler des aspects qui sans elle demeureraient invisibles et dont la profession infirmière aurait, de notre point de vue, tout avantage à prendre la pleine mesure. Car «si le sociologue a un rôle, ce serait plutôt de donner des armes que de donner des leçons" (Bourdieu, 1984b, p. 95). Et la connaissance de l'espace infirmier et des rapports sociaux qui le structurent nous semble pouvoir constituer une arme particulièrement efficace, précisément pour favoriser l'émancipation de la profession.

La prise en compte de la diversité et des inégalités qui composent la profession infirmière passe nécessairement par l'intégration de ces thématiques dans l'enseignement. Visualisables sous forme de schémas, l'espace infirmier, l'espace des positions, les quatre fractions ainsi que l'échelle de prestige des secteurs peuvent facilement être présentés aux étudiantes et étudiants, dans une perspective à la fois pragmatique et critique. Pragmatique, car en visualisant cette sorte de "photographie» de la profession et les enjeux qui caractérisent les différents secteurs, les élèves peuvent non seulement adosser le choix de leur futur lieu de pratique à des informations concrètes, mais aussi anticiper le type de trajectoire dont ce lieu de pratique pourrait être le point de départ. Critique, car l'espace infirmier ne devrait jamais être considéré comme un simple "manuel d'orientation" permettant de repérer les "meilleurs secteurs", mais plutôt comme un état transitoire de rapports de force qu'il est possible de changer. Dans cette perspective, c'est aux enseignantes et enseignants qu'il revient de ne jamais céder à "la tentation de transformer des lois ou des régularités historiques en lois éternelles" (BOURDIEU, 1984b, p. 95) : les tendances que révèle notre recherche ont beau s'inscrire dans une histoire longue, elles n'en gardent pas moins un caractère contingent, et peuvent de ce fait être contestées 
et modifiées. C'est donc en intégrant pleinement l'étude des rapports de pouvoir dans la formation en soins que la profession infirmière aura les meilleures chances de modifier les rapports de pouvoir qui la traversent et d'éviter toute nouvelle mise sous tutelle. 



\section{Postface de Patrice Pinell}

L'ouvrage de Philippe Longchamp, Kevin Toffel, Felix Bühlmann et Amal Tawfik est l'aboutissement d'une recherche sur la profession infirmière qui fera date pour tous ceux qui s'intéressent à la sociologie de la santé et cela pour au moins deux raisons. D'abord pour la façon, parfaitement maitrisée, dont les auteurs articulent le maniement des concepts avec un travail d'enquête pour lequel ils mobilisent les principaux outils méthodologiques de la discipline. Ensuite pour leur ambition théorique de sortir des chemins balisés de la sociologie des professions pour mettre en œuvre une approche inspirée par la théorie des champs de Pierre Bourdieu. Le défi n'était pas mince tant, depuis Parsons, l'univers des travaux sur les professions de santé est historiquement dominé par les productions des grands courants de la sociologie nord-américaine. Ce sont elles, d'ailleurs, qui ont orienté les recherches menées, en France notamment, sur les groupes professionnels des infirmières et des aide-soignantes. C'est pourquoi l'intérêt de ce livre, loin de se limiter aux connaissances qu'il apporte sur les infirmières de Suisse romande, repose aussi à mes yeux sur le pari, réussi, de montrer l'intérêt heuristique de l'utilisation du concept de champ. L'analyse proposée aura permis de mettre en évidence des choses qui échappaient aux recherches menées jusqu'à présent sur ce groupe professionnel. 
La démarche suivie par les auteurs montre leur souci d'éviter les travers du théoricisme. Leur texte ne s'ouvre pas sur un exposé synthétisant ce qu'il en serait de la théorie des champs, mais par une interrogation sur les différents usages sociologiques du concept qui ont été fait depuis les travaux princeps de Bourdieu et en ont étendu le domaine d'application. Comme ils le soulignent à juste titre, Bourdieu construit son cadre conceptuel «champ/ habitus» dans une série de recherches portant sur des groupes sociaux engagés chacun dans des activités relativement spécifiques - scientifiques, juristes, ecclésiastiques, représentants politiques, écrivains, grands couturiers, industriels, économistes -, mais qui ont en commun d'appartenir aux classes dominantes. Son ambition intellectuelle est d'élaborer une théorie du pouvoir, qu'il n'aura pas eu le temps de mener à son terme, laissant en friche son analyse de ce "champ des champs" qu'il appelait le champ du pouvoir. D'où l'interrogation, quelque peu rhétorique, des auteurs sur ce qu'il pourrait y avoir de paradoxal dans leur projet de mettre en œuvre une analyse en termes de champs pour rendre compte d'un groupe professionnel que le sens commun et la sociologie classent dans la catégorie des groupes socialement dominés. Rhétorique parce que leur idée, dans la suite de réflexions que j'avais esquissées (PINELL, 2012), était précisément de regarder de plus près les usages possibles du concept dans sa version «incomplète». Du cadre conceptuel élaboré par Bourdieu, on peut distinguer la démarche méthodologique - à savoir la mise en ouvre d'une analyse structurale et relationnelle des positions occupées par les agents dans le champ étudié - du travail théorique de définition des propriétés qui caractérise ce qu'est un champ. La démarche méthodologique est un outil sociologique pour rendre compte de la production par des agents particuliers de biens symboliques et/ou matériels spécifiques de tous ordres (des biens culturels, des biens de salut, des normes juridiques, des produits de haute couture, des réformes politiques...). La production de ces biens spécifiques est ainsi analysée comme le produit d'un espace social formé par les positions de leurs producteurs, sachant que ces positions ne peuvent être définies et étudiées que dans 
leurs interrelations. Mais les espaces sociaux ainsi construits ne sont considérés par Bourdieu comme des champs que s'ils possèdent, en outre, un ensemble de caractéristiques, la plus discriminative étant de disposer d'un haut degré d'autonomie par rapport aux autres champs avec lesquels ils sont en relation. Même si l'autonomie d'un champ est toujours relative et qu'il n'est pas imperméable aux logiques de fonctionnement d'autres champs (en particulier à celle du champ économique), ce qui caractérise un champ, c'est sa capacité à réfracter, à retraduire les logiques extérieures qui s'exercent sur lui, dans sa propre logique ${ }^{120}$. À l'aune de ce critère d'autonomie, le présupposé théorique d'un "champ infirmier» apparaît d'emblée pour le moins discutable. Parce qu'en toute rigueur théorique, il implique l'idée d'une autonomie vis-à-vis du champ médical, ce qui signifie l'existence d'une production symbolique spécifique, d'un savoir infirmier irréductible à toute autre forme de savoir, à la fois indépendant du savoir médical et des savoirs produits par d'autres disciplines scientifiques (sciences humaines et sociales notamment). Un tel présupposé va trop à l'encontre des résultats de toute une littérature centrée sur l'étude des formes de domination sociale et symbolique subies par les infirmières, pour ne pas être écarté, au moins au départ, par les auteurs. Mais il leur reste - et c'est ce qui les intéresse - la possibilité de construire leur objet d'étude en se servant du concept de champ dans sa modalité "faible», dans sa dimension d'outil méthodologique, pour analyser le

${ }^{120}$ Ainsi, la définition de la bonne littérature, et partant la hiérarchisation symbolique des écrivains, se fait sur des critères propres au champ littéraire et non sur des critères économiques. Si la logique du champ économique est très influente dans le pôle dit de "littérature commerciale», ce pôle reste un pôle dominé du champ et ses productions sont, la plupart du temps, un objet de mépris pour les écrivains dominants. Il en va tout autant de la production de biens de santé comme la définition des entités cliniques, de leur diagnostic et leur traitement qui dans une très large mesure, restent spécifiques au savoir médical. Même si ces biens de santé sont susceptibles de générer des intérêts économiques, la production de ces biens symboliques obéit à une logique avec ses normes et ses règles qui n'est pas la logique de production du champ économique. 
groupe professionnel des infirmières. Dans cette perspective, le groupe est traité sociologiquement comme un espace social de production de biens spécifiques, des «biens infirmiers» (dont la nature et les propriétés restaient en partie à préciser, ce que fera leur recherche). Un espace qui est aussi et indissociablement un espace de lutte pour la définition de ce que sont les spécificités de la profession infirmière, à la fois dans ses savoirs, ses pratiques, ses relations avec les autres soignants, en premier lieu les médecins et les patients. Et cette prise de position méthodologique est assortie de l'hypothèse qu'il s'agit d'un espace dominé du champ médical. Toutefois, il ne s'agit pas d'une hypothèse figée dans les représentations de ce qu'avait pu être la domination des médecins sur les infirmières durant la majeure partie du siècle passé. Une reconstruction historique est entreprise, qui montre les évolutions de la place occupée par le groupe professionnel au sein du champ médical. Celles qui touchent à la formation (son "académisation" et la création de masters en soins infirmiers) et aux statuts, mais aussi celles qui sont la conséquence des transformations du champ médical: spécialisation de plus en plus poussée, part croissante des sciences expérimentales dans la production du savoir, politique néolibérale de rationalisation des coûts budgétaires, nouvelle gouvernance managériale de l'hôpital et tout ce qu'avec Alain Supiot (2015), on peut appeler «la gouvernance par les nombres» et qui sont autant d'éléments modifiant les rapports de pouvoir entre les médecins hospitalo-«universitaires» et l'administration gestionnaire. C'est donc par rapport à ces évolutions que les modalités de la domination s'exerçant sur les infirmières sont interrogées tout au long de la recherche.

Une remarque encore sur les conséquences de la problématique adoptée par les auteurs. Dans la mesure où elle traite la profession infirmière comme un espace de luttes entre des agents occupant des positions spécifiques et qui s'opposent sur la définition de ce qu'est cette profession, une telle problématique interroge, pour la déconstruire, la représentation, dominante dans la société, d'un groupe professionnel homogène. Une représentation, ancrée dans 
une histoire ancienne, d'un groupe dont l'homogénéité tient à ce qu'il est tout entier confondu dans notre sens commun avec son image archétypale de "la femme infirmière auxiliaire de l'homme médecin». Mais il se trouve que cette représentation du groupe comme entité sociale homogène est aussi au cœur du discours de celles qui, se présentant comme les porte-parole des intérêts de la profession, remettent en cause cette conception d'une division du travail médical réduisant la fonction infirmière à un simple auxiliariat. Ces dernières sont souvent les mêmes qui défendent l'idée qu'il existe un savoir infirmier propre, indépendant et complémentaire du savoir médical, ce qui, pour elles, fonde en légitimité la revendication d'autonomie de la profession. Et la connaissance qu'elles peuvent avoir de la sociologie des professions les prédispose à attendre d'une recherche sociologique la confirmation du bien-fondé de leur discours. D'où la difficulté particulière pour les chercheurs de trouver la bonne distance vis-à-vis des enquêtées en évitant les deux écueils de l'objectivation froide - se limitant à renvoyer les infirmières à leur position dominée - et de l'empathie acritique, faisant d'eux des alliés mettant leur expertise sociologique au service de la cause de l'autonomie professionnelle. Une difficulté que les auteurs, et ce n'est pas là leur moindre mérite, ont su résoudre.

Étudier le groupe des infirmières en tant qu'il constitue un espace au sein duquel chacune occupe une position sociale à la fois particulière et en relation avec les positions des autres infirmières, exige pour être fait correctement un important travail empirique. Qu'on en juge: après une phase exploratoire consistant en une vingtaine d'entretiens semi-directifs, les auteurs ont réalisé une enquête par questionnaire portant sur un échantillon représentatif de près de 3000 infirmières de Suisse romande exerçant dans 32 secteurs d'activités, services hospitaliers, établissements extrahospitaliers, structures de direction et de formation, puis ont complété leurs données par une dizaine d'entretiens avec des infirmières titulaires d'un master en soins. Ce dispositif méthodologique a permis 
de recueillir des données, d'une part sur les postes occupés et les trajectoires sociales et professionnelles, d'autre part sur les représentations que chaque infirmière se fait de la profession, de ses valeurs, des qualifications qu'elle exige, du degré de prestige attaché aux différents secteurs d'activité, de sa propre place dans la hiérarchie de la profession, de son autonomie dans le travail, des rapports avec l'encadrement, avec les médecins...

Parce que l'occupation d'une position donnée dans un espace social quel qu'il soit, est liée à la possession par l'agent d'un certain type et d'une certaine quantité de ressources (de capital) rendant possible son accession à cette position, il revenait aux auteurs de repérer la "nature» de ces ressources et trouver les moyens d'évaluer leur importance. Ceux-ci ont identifié deux formes de capital culturel particulièrement efficient (et donc valorisé) dans l'espace infirmier, le "capital médical» et le "capital infirmier». Le premier est un capital spécifique au champ médical dont l'importance est un élément déterminant de la hiérarchisation symbolique et sociale du champ. Non seulement il n'est pas spécifique à l'espace infirmier, mais les ressources qui le composent - connaissances en physiopathologie, en pharmacologie, maîtrise de gestes techniques impliquant très souvent la manipulation d'instruments et de machines - ne font pas partie de celles qui, relevant des compétences à poser un diagnostic, sont les plus valorisées dans le champ médical. Autrement dit, le fait qu'une composante du capital médical pas ou peu efficiente pour accéder à des positions dominantes dans le champ médical, puisse l'être dans l'espace infirmier, signe son caractère d'espace dominé. Le second type de capital est qualifié par les auteurs "d'infirmier" avec des guillemets, dans la mesure où, si les ressources auxquelles il renvoie sont mises en avant par les porte-parole de la profession infirmière comme des formes spécifiques de savoir et de pratiques, elles empruntent pour l'essentiel aux sciences humaines et sociales et/ou aux dites «sciences» du management et de gestion. 
La construction del'espace infirmier par la méthode de l'analyse des correspondances multiples donne à voir l'hétérogénéité du groupe professionnel. La répartition des positions dans l'espace montre un premier principe de différenciation selon qu'elles correspondent à une prédominance du «capital médical» sur le «capital infirmier» ou à la prédominance inverse. Cette ligne de partage se combine avec un second principe de différenciation selon le volume et le type des capitaux requis. Il ressort au final une partition de l'espace en quatre groupes. Là où le capital médical prévaut, une bipolarisation tend à différencier entre des positions "dominantes" requérant un volume élevé de capital et des positions "dominées» où ce volume est faible. Les premières correspondent à des positions dans les services hospitaliers considérés par les infirmières comme les plus prestigieux - réanimation, urgence, chirurgie - les secondes renvoient aux services "dépréciés», de moindre technicité comme la gériatrie, la gynécologie ou encore la pédiatrie. Dans le cas des positions à "capital infirmier», la bipolarisation obéit à une logique quelque peu différente. Elle oppose les positions des "élites infirmières» - cadres gestionnaires, enseignantes, infirmières "recherche» supposant un volume important de "capital infirmier» légitime - diplôme post-gradué en sciences infirmières, compétences en gestion, en recherches en soins infirmiers - à des positions compatibles avec un capital infirmier "hétérodoxe» fait de savoirs et de pratiques se référant à l'univers des sciences humaines et sociales et des médecines dites parallèles. Positions que l'on retrouve dans les secteurs extrahospitaliers, entre autres du médico-social et des soins à domiciles.

Un reproche que l'on a pu faire à certaines analyses se référant à la théorie des champs est de ne présenter qu'une image figée de la réalité étudiée (la métaphore de la photographie étant alors convoquée). On peut dire qu'ici, il n'en est rien. En effet, l'étude des trajectoires individuelles d'infirmières anime le tableau d'un espace organisé autour de quatre pôles. Elle rappelle non seulement que l'occupation d'une position donnée est inscrite 
dans une évolution de carrière, mais elle éclaire aussi sur les différentes stratégies qui conduisent nombre d'entre elles à circuler dans l'espace infirmier, comme sur le sens que celles-ci donnent à leur parcours professionnel. De fait, dans leur majorité (et en rapport avec leur ancienneté) les infirmières ont changé au moins une fois (souvent deux, parfois même trois) de secteur de l'espace. Mais tous les changements de position ne se font pas avec la même fréquence. Dans la mesure où la grande majorité des carrières commence à l'hôpital, l'occupation d'une position d' "élite infirmière» ou d' "hétérodoxe» vient le plus souvent après un passage par un service hospitalier. En revanche, le passage d' «élite infirmière» à "dominante médicale» reste exceptionnel. De même, les trajectoires menant d'un secteur hospitalier au pôle hétérodoxe extrahospitalier sont beaucoup plus fréquentes que celles allant dans le sens inverse. $\mathrm{Si}$, en conséquence, une fois sorti de l'hôpital, on n'y revient pas, c'est que cette sortie de l'hôpital vient très souvent sanctionner chez les infirmières un désir de modifier le cours de leur existence, en changeant de cadre institutionnel, pour trouver des conditions de travail moins contraignantes, moins soumises à la hiérarchie, permettant de combiner plus harmonieusement leur vie professionnelle avec leur vie privée. Ce passage vers le pôle «hétérodoxe» semble aussi aller de pair avec des dispositions à contester l'ordre hospitalier dont la manifestation a pu se traduire par des rapports difficiles avec l'encadrement ou les médecins. Mais il est intéressant de voir que le passage du secteur dominant "médical» à une position extrahospitalière, se fait très rarement de façon directe. Sans doute parce que les conditions pour un tel choix sont rarement réunies en ce qu'il implique, pour l'infirmière, une prise de distance par rapport à la hiérarchie des valeurs que, jusque-là, elle faisait sienne. C'est pourquoi, comme le montre la recherche, la trajectoire se fait en deux temps, avec un séjour intermédiaire dans un service de faible prestige du pôle médical dominé.

L'analyse proposée dans l'ouvrage permet également de rendre compte d'une évolution en cours, liée à l'impact sur 
le groupe professionnel de la réforme inscrivant les études en soins dans un cadre universitaire et créant des facultés de soins infirmiers avec, à la clef, des possibilités de carrières académiques. Les auteurs portent une attention particulière à l'émergence récente d'une nouvelle sous-catégorie des élites infirmières, distincte des cadres gestionnaires - avec qui elles peuvent être en opposition - et qui a vocation à développer l'enseignement et la recherche en soins infirmiers. Encore très minoritaire, mais se distinguant par la proportion nettement supérieure d'agents issus des classes supérieures ou moyennes supérieures, ce sous-groupe occupe des positions qui, du fait des fonctions qui leur sont attachées, peuvent être déterminantes pour l'avenir de la profession. Les activités de ce sous-groupe combinent la transmission des connaissances, la production de travaux en "sciences infirmières", ainsi que la surveillance des «bonnes pratiques» cliniques ayant pour finalité une meilleure prise en charge des patients (démarche qualité). D'où l'hypothèse qui s'ouvre à la fin de l'ouvrage et fait des "élites infirmières» le possible vecteur d'un ancrage des logiques du New Public Management au sein de l'ethos professionnel infirmier, à défaut d'être le pôle de production d'un savoir infirmier spécifique et autonome tant par rapport au savoir médical que par rapport à celui des sciences humaines. Objectif qui, à l'heure actuelle, continue de poser problème. 



\section{Remerciements}

Appréhender la profession infirmière dans son ensemble à l'échelle de la Suisse romande est un objectif ambitieux qui n'aurait pu être atteint sans l'appui et la générosité de nombreuses personnes.

En acceptant de nous accorder un entretien au tout début de cette recherche, Dominique Bidet-Dazin (Haute École de Santé Genève) et Anne Gerber (Haute École de Santé Vaud) nous ont grandement aidés à nous familiariser avec le monde complexe des soins infirmiers. Elles ont ainsi joué pour nous le rôle d'«informatrices privilégiées». Qu'elles en soient ici chaleureusement remerciées. Nous tenons également à remercier Isabelle Gindrat (Haute École de Santé Arc), qui nous a transmis plusieurs contacts pour la phase des entretiens.

Lors de l'élaboration d'un questionnaire, rédiger des questions qui ont du sens pour les sociologues est une chose; en rédiger qui ont du sens pour les personnes auxquelles il s'adresse en est une autre. Nous adressons nos plus vifs remerciements à nos collègues Annick Anchisi, Véronique de Goumoëns, Michèle Lilla, Murielle Pott, Corinne Schaub et Alexia Stantzos pour leur relecture attentive de la première version de notre questionnaire, et pour leurs remarques et commentaires qui nous ont permis de grandement améliorer la formulation de certaines questions. 
La saisie des questionnaires papier est un travail important qui dépasse de loin une simple exécution. Nous tenons à remercier Johanna Anglade, Shadya Monteiro, Katia Tâche et Benoît Pierroz, étudiants de la filière soins infirmiers de HESAV, pour avoir contribué à la réussite de cette étape de la recherche.

Nous remercions les directions des soins des différentes institutions romandes qui ont activement collaboré à la diffusion de notre questionnaire. L'Association Suisse des Infirmières (ASI) nous a également été d'une grande aide, et nous remercions en particulier Roswitha Koch pour son ouverture et son appui.

C'est grâce au financement octroyé par le Fonds national suisse de la recherche scientifique (subside n ${ }^{\circ} 100017 \_146042$ ) et aux infrastructures offertes par la Haute École de Santé Vaud (HESAV) et l'Université de Lausanne (UNIL) que ce travail a pu être réalisé dans d'excellentes conditions. Nous adressons des remerciements particuliers à Christine Pirinoli, responsable de la recherche à HESAV de 2008 à 2018, pour avoir toujours cru en ce travail.

Enfin, nous tenons à remercier toutes celles et tous ceux qui, réduit.e.s à l'anonymat, ont accepté de répondre à notre enquête: les 31 infirmières et infirmiers qui ont partagé avec nous leurs expériences et connaissances de la profession à l'occasion de longs entretiens, ainsi que les 3935 personnes qui se sont donné la peine de remplir notre questionnaire. 


\section{Bibliographie}

Аввотт, A. (1988). The systeme of professions. An essay on the division of expert labor. Chicago: University of Chicago Press.

Acker, F. (1995). Informatisation des unités de soins et travail de formalisation de l'activité infirmière. Sciences sociales et santé, 13(3), 69-92.

ACKer, F. (2005). Les reconfigurations du travail infirmier à l'hôpital. Revue française des affaires sociales, 1(1), 161-181.

Addor, V., Schwendimann, R., Gauthier, J.-A., Wernli, B., Jäckel, D., \& Paignon, A. (2016). L'étude nurses at work: Parcours professionnels des infirmières/infirmiers au cours des 40 dernières années en Suisse. Bulletin de l'Obsan, (8).

Allen, D. (2014). Re-conceptualising holism in the contemporary nursing mandate. From individual to organisational relationships. Social science \& medicine, 119, 131-138.

Andersson, E. P. (1993). The perspective of student nurses and their perceptions of professional nursing during the nurse training programme. Journal of Advanced Nursing, 18, 808-815.

Barlet, M., \& Cavillon, M. (2011). La profession d'infirmière: Situation démographique et trajectoires professionnelles. Paris: DREES.

Bastien, C., \& Rick, O. (2009). Hiérarchie des spécialités (para)médicales et marginalisation des vieux. TRANSEO, 1. Consulté à l'adresse http://www.transeo-review.eu/Hierarchie-des-specialites-para.html 
BASZANGer, I. (1986). Les maladies chroniques et leur ordre négocié. Revue française de sociologie, 27(1), 3-27.

Belorgey, N. (2010). L'hôpital sous pression: Enquête sur le "nouveau management public». Paris: La Découverte.

Blanchet, A. \& Gotman, A. (2001). L'enquête et ses méthodes: l'entretien. Paris : Nathan.

Boltanski, L. (1969). Prime éducation et morale de classe. La Haye: Mouton \& Cie.

Bourdieu, P. (1974). Avenir de classe et causalité du probable. Revue française de sociologie, 15(1), 3-42.

Bourdieu, P. (1978). Classement, déclassement, reclassement. Actes de la recherche en sciences sociales, 24, 2-22.

Bourdieu, P. (1979a). La Distinction. Critique sociale du jugement. Paris : Éditions de Minuit.

Bourdieu, P. (1979b). Les trois états du capital culturel. Actes de la recherche en sciences sociales, 30, 3-6.

Bourdieu, P. (1984a). Homo Academicus. Paris: Éditions de Minuit.

Bourdieu, P. (1984b). Questions de sociologie. Paris: Éditions de Minuit.

Bourdieu, P. (1989). La noblesse d'État. Grandes écoles et esprit de corps. Paris: Éditions de Minuit.

Bourdieu, P. (1992). Les règles de l'art. Genèse et structure du champ littéraire. Paris: Éditions du Seuil.

Bourdieu, P. (1993). La misère du monde. Paris: Éditions du Seuil.

Bourdieu, P. (1998). La domination masculine. Paris: Éditions du Seuil.

Bourdieu, P. (2003). Méditations pascaliennes. Paris: Éditions du Seuil.

Browne, A. J. (2001). The influence of liberal political ideology on nursing science. Nursing Inquiry, 8(2), 118-129.

Calbéra, J.-B. (2003). La profession infirmière. L'historique et le mythe. Vie sociale et traitements, 79(3), 55-57.

Castra, M. (2004). Faire face à la mort: Réguler la «bonne distance» soignants-malades en unité de soins palliatifs. Travail et Emploi, 97, 53-64. 
Chambaz, C., Maurin, E., \& Torelli, C. (1998). L'évaluation sociale des professions en France. Construction et analyse d'une échelle des professions. Revue française de sociologie, 39(1), 177-226.

Chang, P.-L., Chou, Y.-C., \& Cheng, F.-C. (2006). Designing career development programs through understanding of nurses' career needs. Journal of nurses in staff development, 22(5), 246-253.

Chapman, C. M. (1982). Degrees in nursing. In P. Allan \& M. Jolley (Éd.), Nursing, Midwifery \& Health Visiting Since 1900, p. 174-179. Londres: Faber and Faber.

Chaves, G. (2005). Étude sur l'image que la profession "infirmière» a d'elle-même. Lausanne: Service de la Santé publique de l'État de Vaud.

Chinn, P., \& Kramer, M. (2008). Integrated theory and knowledge development in nursing. St. Louis: Elsevier.

Collière, M.-F. (1982). Promouvoir la vie. De la pratique des femmes soignantes aux soins infirmiers. Paris: Interéditions.

Dallaire, C. (2015). La difficile relation des soins infirmiers avec le savoir. Recherche en soins infirmiers, 121(2), 18-27.

De Certeau, M. (1980). L'invention du quotidien. Paris: Gallimard.

De Montlibert, C. (1980). L'éducation morale des familles. L'extension du métier de puéricultrice. Actes de la recherche en sciences sociales, 32-33, 65-76.

Donaldson, S. K. (2003a). Commentary: The Science of Human Health - Domain Structure and Future Vision. Biological Research for Nursing, 4(3), 165-169.

Donaldson, S. K. (2003b). It's About Health, not Nursing. Journal of Professional Nursing, 19(4), 180-181.

Douguet, F., \& Vilbrod, A. (2007). Le métier d'infirmière libérale. Portrait sociologique d'une profession en pleine mutation. Paris: Éditions Seli Arslan. 
Dreyfus, M. (Éd.). (2009). Les assurances sociales en Europe. Rennes: Presses Universitaires de Rennes.

Droux, J. (2000). L'attraction céleste. La construction de la profession d'infirmière en Suisse Romande (XIX'-XX' siècles), thèse de doctorat en Lettres. Genève: Université de Genève.

Dubet, F. (2002). Le déclin de l'institution. Paris: Éditions du Seuil.

Ehrenreich, B., \& English, D. (1976). Sorcières, sages-femmes et infirmières. Une histoire des femmes et de la médecine. Montréal: Les éditions du remue-ménage.

Elzinga, A. (1990). The knowledge aspect of professionalization. The case of science-based nursing education in Sweden. In R. Torstendahl \& M. Burrage (Éd.), The formation of professions. Knowledge, State and Strategy (p. 151-173). London: Sage Publications.

FAure, O. (1994). Histoire sociale de la médecine en France XVIII'-XXe siècle. Paris: Économica.

Feroni, I., \& Kober, A. (1995). L'autonomie des infirmières. Une comparaison France/Grande-Bretagne. Sciences sociales et santé, 13(3), 35-68.

Feroni, I., \& Kober-Smith, A. (2005). La professionnalisation des cadres infirmiers: L'effet de l'action publique en France et en Grande-Bretagne. Revue française de sociologie, 46(3), 469-494.

Freidson, E. (1963). Medical care and the public: Case study of a medical group. Annals of the American Academy of Political and Social Science, 346, 57-66.

Freidson, E. (2007). Professionnal Dominance. The Social Structure of Medical Care. New Brunswick: Transaction Publishers.

Fusulier, B. (2011). Le concept d'ethos. De ses usages classiques à un usage renouvelé. Recherches sociologiques et anthropologiques, 42 (1), 97-109.

Ganzeboom, H. B. G., \& Treiman, D. J. (1996). Internationally comparable measures of occupational status for the 1988 international standard classification of occupations. Social science research, 25, 201-239.

Gaspar, J.-F. (2012). Tenir! Les raisons d'être des travailleurs sociaux. Paris : La Découverte. 
Giauque, D. (2014). Quête identitaire des cadres intermédiaires hospitaliers. Société Suisse des Sciences Administratives, 20, 97-109.

Gortner, S. R. (2000). Knwoledge Development in Nursing: Our Historical Roots and Future Opportunities. Nurs Outlook, 48, 60-67.

Grignon, C., \& Passeron, J.-C. (1989). Le savant et le populaire. Misérabilisme et populisme en sociologie et en littérature. Paris: Éditions du Seuil.

Hartmann, M. (2007). Eliten und Macht in Europa: Ein internationaler Vergleich. Frankfurt: Campus Verlag.

Holmes, D., \& Perron, A. (2008). L'infirmière praticienne en soins de première ligne: Entre colonisation et affranchissement. In C. Dallaire (Éd.), Le savoir infirmier. Au cour de la discipline et de la profession (p. 403-414). Montréal: Les Éditions de la Chenelière.

Hout, M., \& DiPrete, T. A. (2006). What we have learned: RC28's contributions to knowledge about social stratification. Research in Social Stratification and Mobility, 24, 1-20.

Hughes, A. (2010). The challenge of contributing to policy making in primary care: The gendered experiences and strategies of nurses. Sociology of Health \& Illness, 32(7), 977-992.

Hughes, E. C. (1996). Le regard sociologique. Essais choisis. Paris: EHESS.

Jaccard Ruedin, H., Weaver, F., Roth, M., \& Widmer, M. (2009). Personnel de santé en Suisse-État des lieux et perspectives jusqu'en 2020. Neuchâtel: Observatoire suisse de la santé.

Judd, D. (2010). Nursing in the United States From the 1980s to the Present. New Opportunity, Technical Advances, Nursing Autonomy and Globalization. In D. Judd, K. Sitzman, \& M. Davis (Éd.), A History of American Nursing (p. 180-209). Sudbury: Jones \& Bartlett.

Knibiehler, Y. (2008). Histoire des infirmières en France au XX' siècle. Paris: Hachette.

Lafarge, G., \& Marchetti, D. (2011). Les portes fermées du journalisme. L'espace social des étudiants des formations "reconnues». Actes de la recherche en sciences sociales, 189(4), 72-99. 
Laget, P.-L., \& Salaün, F. (2004). Aux origines de l'hôpital moderne, une évolution européenne. Les Tribunes de la santé, 3(2), 19-28.

Lahire, B. (2001). Le travail sociologique de Pierre Bourdieu. Dettes et critiques. Paris: La Découverte \& Syros.

Lahtinen, P., Leino-Kilpi, H., \& Salminen, L. (2014). Nursing education in the European higher education area-Variations in implementation. Nurse Education Today, 34(6), 1040-1047.

Lambert, V. A., \& Lambert, C. E. (2005). The Evolution of Nursing Education and Practice in the U.S. In J. Daly, S. Speedy, D. Jackson, V. A. Lambert, \& C. E. Lambert (Éd.), Professional Nursing: Concepts, Issues, and Challenges (p. 17-50). New-York: Springer.

Lanza, D., Longchamp, P., Seferdjeli, L., \& Müller, R. (2004). Entre paroles et actions, appréhender la complexité de la coopération en milieu hospitalier. In L. Gajo (Éd.), Langue de l'hôpital, pratiques communicatives et pratiques de soins (p. 97-129). Lausanne: Cahiers de l'ILSL, Vol. 16.

Le Roux, B., \& Rouanet, H. (2010). Multiple Correspondence Analysis. Los Angeles/London/New Delhi/Singapore/Washington DC: SAGE.

LerT, F. (1996). Le rôle propre peut-il fonder l'autonomie professionnelle des infirmières? (Commentaire). Sciences sociales et santé, 14(3), 103-115.

Liberati, E. G. (2017). Separating, replacing, intersecting. The influence of context on the construction of the medical-nursing boundary. Social science \& medicine, 172, 135-143.

Lindsay, S. (2007). Gendering Work: The Masculinization of Nurse Anesthesia. The Canadian Journal of Sociology, 32(4), 429-448.

LongChamp, P. (2009). Des infirmières scolaires dans l'espace social. Carnets de bord en sciences humaines, 16, 45-66.

Longchamp, P., \& Toffel, K. (2016). Un espace infirmier? Éléments pour une analyse structurale de la profession infirmière. Working Paper, Lausanne: Haute École de Santé Vaud: https://www.academia. edu/36087180/Un_espace_infirmier_\%C3\%891\%C3\%A9ments_ pour_une_analyse_structurale_de_la_profession_infirmi\%C3\%A8re. 
Longchamp, P., Toffel, K., Bühlmann, F., \& Tawfik, A. (2016). De la sociologie de l'innovation à l'imagination sociologique. La théorie des champs à l'épreuve de la profession infirmière. Cahiers de recherche sociologique, 59-60, 135-156.

Longchamp, P., Toffel, K., Bühlmann, F., \& Tawfik, A. (2018). L'espace professionnel infirmier. Une analyse à partir du cas de la Suisse romande. Revue française de sociologie, 59(2), 219-258.

Marichalar, P., \& Pitti, L. (2013). Réinventer la médecine ouvrière? Retour sur des mouvements médicaux alternatifs dans la France post-1968. Actes de la recherche en sciences sociales, 196-197(1), 114-131.

Martin, O. (2012). L'enquête et ses méthodes: l'analyse des données quantitatives. Paris: Armand Colin.

Mathieu, L. (2000). L'espace de la prostitution. Eléments empiriques et perspectives en sociologie de la déviance. Sociétés Contemporaines, $38,99-116$.

Matonti, F., \& Poupeau, F. (2004). Le capital militant. Essai de définition. Actes de la recherche en sciences sociales, 155(5), 4-11.

McPherson, K. (2003). Bedside Matters. The Transformation of Canadian Nursing, 1900-1990. Toronto Buffalo London: University of Toronto Press.

Meleis, A. I. (1991). Directions for Nursing Theory Development in the 21st Century. Nursing Science Quarterly, 5(3), 112-117.

Mendy, A. (2018). Recruitment policies of Non-EU/EEA nurses and ethical issues. A comparison of the UK, France, and Switzerland. In M. Czaika (Éd.), High-Skilled Migration: Drivers and Policies (p. 320-336). Oxford: Oxford University Press.

Milburn, P. (2002). La compétence relationnelle: Maîtrise de l'interaction et légitimité professionnelle. Revue française de sociologie, 43(1), 47-72.

Mooney, M., Glacken, M., \& O’Brien, F. (2008). Choosing nursing as a career. A qualitative study. Nurse Education Today, $28,385-392$.

Neveu, E. (2013). Les sciences sociales doivent-elles accumuler les capitaux? Revue française de science politique, 63(2), 337-358. 
OCDE. (2017). Panorama de la santé 2017. Les indicateurs de l'OCDE. Paris: OCDE.

Oesch, D. (2006). Coming to Grips with a Changing Class Structure. An Analysis of Employment Stratification in Britain, Germany, Sweden and Switzerland. International Sociology, 21(2), 263-288.

Oesch, D. (2013). Occupational Change in Europe. How Technology and Education transform the Job Structure. Oxford: Oxford University Press.

OFS. (2007). L'emploi dans le domaine de la santé. Faits et tendances à la lumière des chiffres des recensements des entreprises de 1995 à 2005. Neuchâtel: Office Fédéral de la Statistique.

OFS. (2019). Statistique des frontaliers. Neuchâtel: Office Fédéral de la Statistique.

Orfàli, K. (2002). L'ingérence profane dans la décision médicale: Le malade, la famille et l'éthique. Revue Française des Affaires sociales, 3(3), 103-124.

Paillet, A. (2007). Sauver la vie, donner la mort. Paris: La Disputel Snédit.

Passeron, J.-C. (2006). Le raisonnement sociologique. Un espace non poppérien de l'argumentation. Paris: Albin Michel.

PenefF, J. (1992). L'hôpital en urgence. Étude par l'observation participante. Paris: Métailié.

Petitat, A. (1989). Les infirmières. De la vocation à la profession. Montréal: Éditions du Boréal.

Petitat, A. (1994). La profession infirmière. Un siècle de mutations. In P. Aïach \& D. Fassin (Éd.), Les métiers de la santé. Enjeux de pouvoir et quête de légitimité (p. 227-259). Paris: Anthropos.

Pichonnaz, D., \& Toffel, K. (2018). Pour une analyse dispositionnelle des pratiques professionnelles. Émulations. Revue de sciences sociales, 25, 7-21.

Picot, G. (2005). Entre médecins et personnel infirmier à l'hôpital public: Un rapport social instable. Revue Française des Affaires sociales, 1(1), 83-100.

Pierru, F. (2008). La santé au régime néolibéral. Savoir/Agir, 5(3), $13-24$. 
Pinell, P. (2005). Champ médical et processus de spécialisation. Actes de la recherche en sciences sociales, 1(156-157), 4-36.

Pinell, P. (2008). Qu'en est-il de l'autonomie du champ médical? Savoir/Agir, 5, 57-63.

Pinell, P. (2009). La genèse du champ médical: Le cas de la France (1795-1870). Revue française de sociologie, 50(2), 315-349.

Pinell, P. (2012). À propos du champ médical: quelques réflexions sur les usages sociologiques du concept de champ. In Mauger, G. et Lebaron, F. (Éd.), Lectures de Bourdieu (p. 305-318). Paris: Éditions Ellipses.

Pinto, L. (2007). La vocation et le métier de philosophe. Pour une sociologie de la philosophie dans la France contemporaine, Paris: Seuil.

Poisson, M. (2008). Le pansement et la pensée: Splendeurs et misères du rôle propre. Recherche en soins infirmiers, 93, 56-60.

Price, S. L. (2009). Becoming a nurse. A meta-study of early professional socialization and career choice in nursing. Journal of Advanced Nursing, 65(1), 11-19.

Price, S. L., McGillis Hall, L., Angus, J. E., \& Peter, E. (2013). Choosing nursing as a career. A narrative analysis of millennial nurses' career choice of virtue. Nursing Inquiry, 20(4), 305-316.

Pudal, R. (2016). Retour de flammes. Les pompiers, des héros fatigués? Paris : La Découverte.

RadCliffe, M. (2000). Doctors and nurses: New game, same result. British Medical Journal, 320(7241), 1085.

Resenterra, F., Siggen, M., \& Giauque, D. (2013). Les cadres intermédiaires entre contraintes managériales et défense des identités professionnelles: L'exemple des hôpitaux de Suisse romande. Humanisme et entreprise, 315(5), 1-24.

Sainsaulieu, I. (2012). La mobilisation collective à l'hôpital: Contestataire ou consensuelle? Revue française de sociologie, 53(3), 461-492.

Schwendimann, R., Jäckel, D., Paignon, A., Gauthier, J.-A., Wernli, B., \& AdDor, V. (2016). Étude sur la fidélisation des infirmières: Premiers résultats. Soins infirmiers, (5), 76-77. 
Sena, B. (2017). Professionalization without Autonomy. The Italian Case of Building the Nursing Profession. Professions and Professionalism, 7(3).

Senotier, D. (1992). Les infirmières, image d'une profession. Cent ans d'évolution de la profession. In D. Kergoat, F. Imbert, H. Le Doaré, \& D. Senotier (Éd.), Les infirmières et leur coordination (p. 23-55). Paris: Éditions Lamarre.

Stevens, J. A. (2011). Student nurses' career preferences for working with older people. A replicated longitudinal survey. International Journal of Nursing Studies, 48, 944-951.

Supiot, A. (2015). La gouvernance par les nombres, Institut d'études avancées de Nantes: Arthème Fayard.

Svensson, R. (1996). The interplay between doctors and nurses-A negotiated order perspective. Sociology of Health \& Illness, 18(3), 379-398.

Terraneo, F., Seferdjeli, L., \& Diby, M. (2010). Le «tour de lit» aux soins intensifs: Représentations et prise d'information. Le travail humain, 73(4), 339-359.

Thouvenin, P. (2004). Ethnographie de la formation des infirmières françaises. Regards sociologiques, 29, 35-53.

Toffel, K. (2018). Positions et prises de position dans la profession infirmière. La structuration d'un espace professionnel au prisme de la théorie des champs (Thèse de doctorat en sciences sociales). Lausanne: Université de Lausanne.

Tofrel, K. (2020). Les enjeux symboliques d'un diplôme académique. Reconfiguration des rapports sociaux et nouvelles tensions au sein de la profession infirmière. Revue suisse de sociologie, 46(1), 73-95.

Toffel, K., \& Longchamp, P. (2017). Importer, transformer, diffuser les savoirs infirmiers. Trois figures de la lutte pour l'autonomisation d'une profession. Anthropologie \& Santé, 14. Consulté à l'adresse https://anthropologiesante.revues.org/2536

Toffel, K., \& TAwfik, A. (à paraître). Le new public management et la redéfinition de l'activité infirmière. De la fabrique des nouvelles élites professionnelles. Revue française d'administration publique. 
Treiman, D. J. (1977). Occupational Prestige in Comparative Perspective. New York/San Francisco/London: Academic Press.

Tronto, J. C. (2008). Du care. Revue du Mauss, 32(2), 243-265.

VAssy, C. (1999). Travailler à l'hôpital en Europe. Apport des comparaisons internationales à la sociologie des organisations. Revue française de sociologie, 40(2), 325-356.

VÉGA, A. (1997). Les infirmières hospitalières françaises: L'ambiguïté et la prégnance des représentations professionnelles. Sciences sociales et santé, 15(3), 103-132.

VÉGA, A. (2000). Une ethnologue à l'hôpital. L'ambiguïté du quotidien infirmier. Paris: Éditions des archives contemporaines.

Weber, M. (1992). Essais sur la théorie de la science. Paris: Pocket.

Weber, M. (2003). Le savant et le politique. Paris: La Découverte. 



\section{Annexes}

Cet ouvrage étant destiné à un large public, nous nous sommes efforcés d'alléger l'exposé des aspects techniques de la recherche dans le corps du texte. Ces annexes permettent cependant au lecteur de se faire une idée plus précise de la façon dont les résultats ont été obtenus. Elles contiennent l'exposé des modalités de l'enquête, en particulier la population et son recrutement (annexe 1), les étapes d'opérationnalisation des capitaux médical et infirmier (annexe 2), les différents tableaux qui présentent les statistiques mobilisées dans l'ouvrage (annexe 3) et finalement l'ACM de l'espace infirmier, accompagnée des tableaux des variables et modalités actives et supplémentaires (annexe 4).

\section{Annexe 1. L'enquête}

L'enquête a été conduite en Suisse romande durant cinq années, de 2012 à 2017. Dans un premier temps, nous avons mené des entretiens semi-dirigés avec 21 infirmières au printemps 2012 (16 femmes et 5 hommes). Âgées de 24 à 55 ans et exerçant dans les 7 cantons de Suisse romande, elles ont été sélectionnées de manière à couvrir les secteurs les plus emblématiques de la 
profession $^{121}$. D'une durée comprise entre 1 et 2 heures, les entretiens se sont déroulés sur les lieux professionnels. Ils ont été intégralement retranscrits et soumis à une analyse thématique catégorielle (Blanchet \& Gotman, 2001).

C'est sur la base des résultats issus de l'analyse des entretiens que nous avons élaboré un questionnaire. Celui-ci a d'abord été testé auprès d'infirmières exerçant à la Haute École de Santé Vaud (HESAV), puis auprès de plusieurs infirmières qui avaient participé à notre enquête par entretiens. Il a ensuite été distribué auprès d'un échantillon représentatif d'infirmières de Suisse romande. La récolte des données s'est déroulée de mai à septembre 2014. Comme il n'existe pas en Suisse de registre statistique regroupant l'ensemble de la population infirmière, nous avons dû renoncer à un échantillonnage aléatoire et privilégier un échantillonnage par grappes qui permet précisément de pallier l'absence d'une base de sondage complète (MARTIN, 2012). Dans un premier temps, nous avons construit une liste des institutions susceptibles d'employer des infirmières en Suisse romande. À l'exception des Établissements médicaux sociaux (EMS) (institutions nombreuses et structurellement équivalentes dont nous avons sélectionné une dizaine par canton) et des cabinets médicaux (que nous n'avons pas directement contactés) ${ }^{122}$, les autres institutions ont été sélectionnées de manière exhaustive. Soit l'ensemble des hôpitaux universitaires, cantonaux et régionaux (auxquels sont rattachés certains secteurs carcéraux ainsi que la plupart des centres de traitement et de réadaptation), des services d'aide et

\footnotetext{
${ }^{121}$ Les infirmières interviewées exercent dans les secteurs suivants: psychiatrie, anesthésie, soins à domicile, médecine, centre de traitement des addictions, éducation, oncologie, établissement médico-social, soins palliatifs, soins intensifs, milieu carcéral, soins continus, urgences adultes, urgences pédiatriques, indépendante, néonatologie, chirurgie, foyer pour requérants d'asile, santé scolaire et institut universitaire.

${ }^{122}$ Les 33 infirmières de cabinet médical qui figurent dans notre échantillon ont été sollicitées par le mailing de l'ASI.
} 
de soins à domicile, des associations d'infirmières indépendantes et d'infirmières d'entreprise, des cliniques privées, des centres médicaux, des services de santé scolaire ou de la jeunesse, des institutions sociales et éducatives, des laboratoires d'analyses et des filières de formation et de recherche. Notre liste comprenait 292 institutions au total. Parmi ces institutions, 66 nous ont déclaré ne pas employer d'infirmières. Sur les 226 institutions restantes, 157 (soit 69,5\%) ont répondu favorablement à notre demande, 62 n'y ont pas donné suite malgré un rappel de notre part, et 7 l'ont déclinée. L'ensemble des infirmières exerçant dans les institutions ayant répondu favorablement ont reçu notre questionnaire (en version papier ou en ligne) ${ }^{123}$ via les directions d'établissement ou les directions de soins.

Parallèlement à cet échantillonnage, l'Association suisse des infirmières (ASI) a procédé à un mailing auprès de tous ses membres francophones, soit 2738 personnes. Un flyer invitant à remplir le questionnaire a également été joint à la revue de l'ASI (5000 exemplaires), et un texte d'annonce a été publié sur le site internet ainsi que sur la page Facebook de l'association. Au total, 3935 questionnaires ont été récoltés. Les individus présentant trop de données manquantes sur les questions prioritairement exploitées pour l'analyse ainsi que ceux livrant systématiquement la même réponse à des séries de questions ont été éliminés ${ }^{124}$. Au total, les analyses présentées ici portent sur 2923 individus. Les institutions qui nous ont communiqué le nombre d'infirmières

\footnotetext{
${ }^{123}$ Bien que coûteuse (frais postaux, déplacements, saisie manuelle), la version papier du questionnaire a probablement contribué à une meilleure représentativité de notre échantillon. Les $10 \%$ d'infirmières qui ont opté pour ce mode de remplissage présentent en effet des caractéristiques spécifiques par rapport à celles qui ont répondu en ligne: plus jeunes, plus souvent issues du secteur privé et plus souvent de nationalité étrangère.

${ }^{124}$ Une comparaison entre l'échantillon retenu pour les analyses et l'échantillon des individus éliminés montre que ce dernier ne représente que très peu de différences sous le rapport de l'âge (+ 2,2 ans), de la proportion d'hommes (1 point de moins), de la proportion d'étrangers (1,8 point de plus) et de la répartition dans les différents secteurs (écart moyen de 0,7 point).
} 
auxquelles elles ont distribué notre questionnaire nous permettent d'estimer le taux de réponse individuel entre 35 et $40 \%{ }^{125}$.

Bien que nous ne disposions pas de point de comparaison, on peut émettre l'hypothèse d'une certaine sous-représentation des infirmières de cabinet médical, les seules à ne pas avoir été contactées directement par nos soins. Il est aussi possible que les infirmières affiliées à l'ASI soient surreprésentées du fait que certaines d'entre elles ont pu recevoir plusieurs sollicitations (par leurs directions, par le mailing de l'ASI ainsi que par le flyer joint à la revue de l'association).

Tous les secteurs de la profession sont représentés dans l'échantillon (nous en distinguons 32 au total, voir tableau A1 en annexe 3). La majorité se situe en milieu hospitalier (70,6\% des individus). Parmi les secteurs extrahospitaliers, on peut mentionner les EMS (5,5\%), les soins à domicile (6\%), la santé scolaire (4,6\%), l'enseignement et la recherche (4,0\%), l'établissement socio-éducatif $(2,2 \%)$ ou encore la pratique indépendante $(1 \%)^{126}$.

Après avoir mené les analyses quantitatives, il est apparu souhaitable d'avoir des données complémentaires que l'enquête par questionnaire n'avait pas permis d'obtenir, notamment concernant une sous-population de la profession: celle des infirmières qui ont suivi une formation de type master en sciences infirmières. Par un retour à une stratégie qualitative, dix entretiens semi-directifs avec des infirmières au bénéfice d'un master acquis à l'Institut universitaire de formation et de recherche en soins (IUFRS) ont

\footnotetext{
${ }^{125}$ Après élimination des questionnaires non exploitables, notre échantillon compte $35,4 \%$ (102 individus sur un total de 288) des infirmières qui exercent en HES dans les cantons de Genève, Neuchâtel, Fribourg et Vaud, 35,5\% (973 sur 2 738) des membres francophones de l'ASI, 40,9\% (135 sur 330) des infirmières scolaires de Romandie et 42,6\% (49 sur 115) des infirmières d'entreprise de Romandie.

${ }^{126}$ Remarquons que la France compte une proportion nettement plus élevée d'infirmières libérales - $15 \%$ selon Florence Douguet et Alain Vilbrod (2007) - que la Suisse romande où les soins à domicile se sont surtout développés sous une forme salariée.
} 
été menés de décembre 2016 à février 2017. Ces infirmières ont été sélectionnées en raison de l'académisation de la profession qu'elles incarnent tout particulièrement. On peut en effet faire l'hypothèse que ces infirmières font figure d'un certain «avenir de la profession». Il était donc important de recueillir des informations, notamment sur leurs motivations à entreprendre le master, leur conception du métier et de l'avenir de la profession, et enfin sur le contenu de leur travail quotidien. Afin d'avoir des profils aussi différents que possible, les infirmières ont été sélectionnées en fonction de leur secteur d'activité et de leur responsabilité d'abord, de leur sexe ensuite et de leurs régions d'exercice enfin. Les entretiens ont donc été effectués avec des infirmières travaillant en tant qu'enseignantes dans les Hautes écoles de santé (4), des infirmières cliniciennes spécialisées (4), une cadre supérieure (1) et une gestionnaire (1). On précisera enfin que deux hommes font partie de ces interviewées qui exercent dans trois cantons (Vaud, Genève, Fribourg).

\section{Annexe 2. De la théorie à la mesure. Opérationnalisation des capitaux médical et infirmier}

Une fois les capitaux médical et infirmier définis, il nous fallait préciser la manière dont nous entendions les opérationnaliser, c'est-à-dire les décomposer en questions concrètes et mesurables soumises aux infirmières dans le cadre de notre questionnaire. Notre démarche s'inspire pour bonne partie de celle proposée par Pierre Bourdieu (1979b) pour le capital culturel ${ }^{127}$. Trois dimensions ont été retenues (Figure A1).

${ }^{127}$ Des trois dimensions du capital culturel identifiées par P. Bourdieu, seule la dimension incorporée (manières de se tenir, de marcher, de parler, de percevoir et ressentir) est absente de notre modèle quantitatif. Ceci s'explique par la difficulté à la mesurer par un questionnaire (elle supposerait des indicateurs tels que l'habileté, la dextérité, les "tours de main", les schèmes de perception visuels, olfactifs et sensitifs, etc.). 
Figure A1. Dimensions et indicateurs des capitaux médical et infirmier

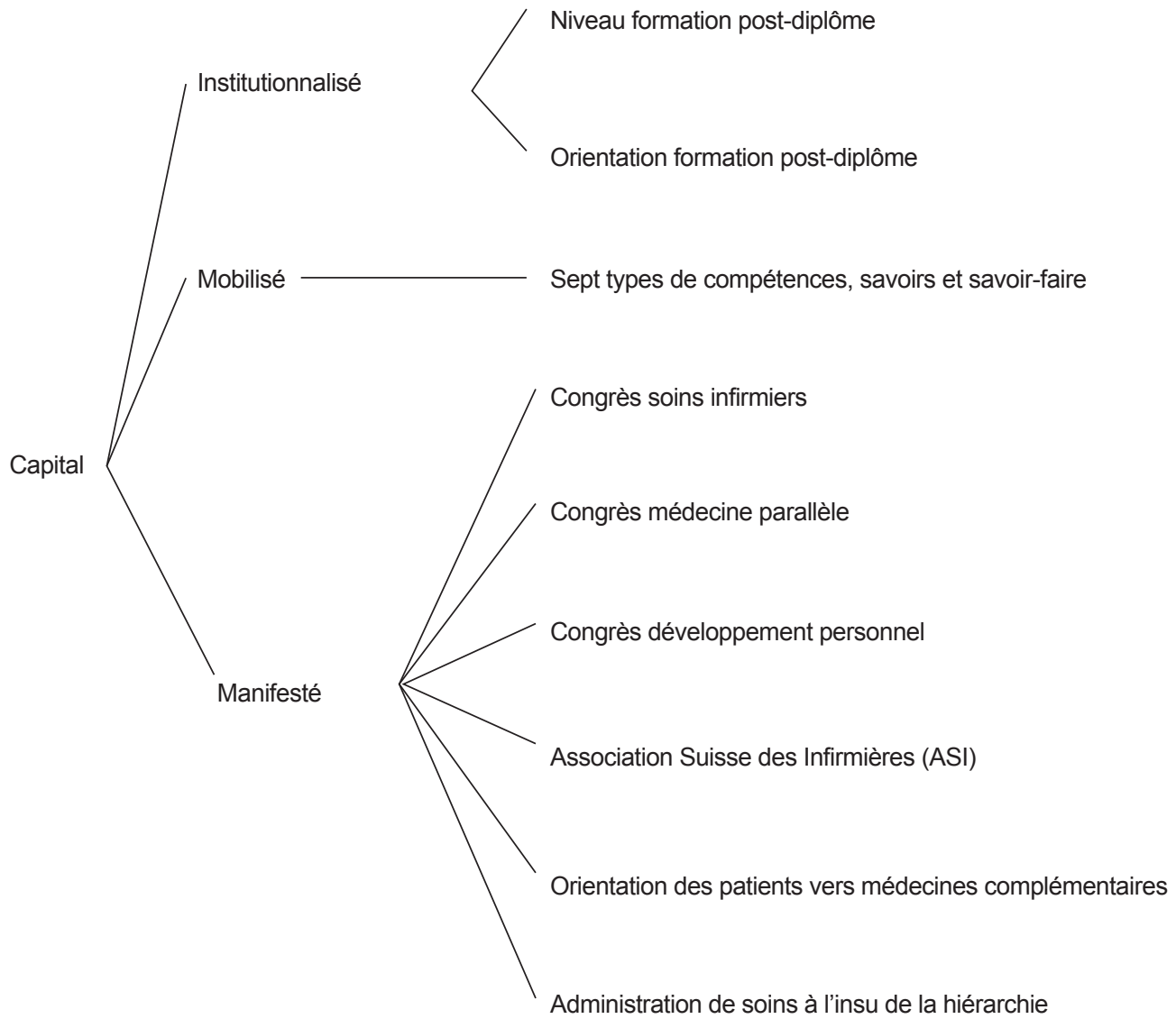

La première est la dimension institutionnalisée, identique à celle identifiée par Pierre Bourdieu pour le capital culturel. Nous retenons comme indicateur la formation la plus élevée suivie par les infirmières après leur diplôme de base. Cette formation est considérée d'une part selon son niveau que nous avons défini à partir d'une question ouverte codée en quatre niveaux: base (aucune formation suivie après le diplôme de base), court (formation dont la durée n'excède pas quelques jours), long (Diploma of Advanced 
Studies, Certificate of Advanced Studies ou assimilés), et académique (bachelor, master, doctorat). La formation est d'autre part considérée selon son orientation définie sur la base d'une question ouverte codée en six orientations: très médicalisée (advanced cardiac life support, anesthésie, soins intensifs, etc.); moyennement médicalisée (hygiène hospitalière, médecine tropicale, soins palliatifs, santé scolaire, etc.); recherche et enseignement (méthodes de recherche clinique, sciences infirmières, etc.); management et encadrement (management, gestion d'équipe, administration publique, etc.); paramédical, développement personnel et religion (aromathérapie, hypnose, "affirmation de soi", "mindfulness", accompagnement pastoral, spiritualité dans les soins, etc.); psychologie et social (psychanalyse, thérapie cognitive et comportementale, philosophie, sciences sociales, "interculturalité», gestion de la violence, santé communautaire, etc.).

La deuxième est la dimension mobilisée. Les indicateurs sont issus d'une même question demandant aux infirmières de préciser à quelle fréquence elles mobilisent une liste de sept compétences, savoirs et savoir-faire qui, sans prétendre à l'exhaustivité, sont néanmoins typiques du travail infirmier. Parmi ces sept variables, deux se rapportent au capital médical et cinq, au capital infirmier. Les indicateurs de capital médical sont les connaissances en physiopathologie et en utilisation de machines ou appareils techniques. Ceux pour le capital infirmier sont les connaissances en modèles théoriques de soins infirmiers, en recherche (bases de données, analyses d'articles, transfert de connaissances), la mobilisation des compétences relationnelles, la mobilisation de l'intuition et la mobilisation des valeurs personnelles. Quatre modalités de réponse ("jamais», "parfois», "souvent» et «toujours») étaient proposées pour chacune des variables. En raison d'une faible sélection de la modalité «jamais", nous avons procédé à un recodage en trois modalités: "rare» (qui recouvre les modalités «jamais» et "parfois»), «souvent» et "toujours». Seule la question relative aux compétences relationnelles a fait l'objet d'un codage différent: les réponses étant concentrées sur les modalités «souvent» et «toujours», nous avons procédé à 
un codage en deux modalités, "plus» (qui recouvre la modalité "toujours») et "moins" (qui recouvre les trois autres modalités). Cette dimension est proche de la dimension objectivée du capital culturel tel que défini par Bourdieu, puisque l'un de ses indicateurs renvoie à des machines et appareils techniques. Elle s'en distingue cependant par le fait qu'elle renvoie à l'usage de ces objets, et non à leur possession (ce qui n'aurait pas de sens dans un contexte de travail le plus souvent salarié, où les individus ne sont pas propriétaires des objets qu'ils utilisent).

Parce qu'en voie de constitution, le capital infirmier intègre une dimension contestataire de l'ordre médical orthodoxe, et est par conséquent faiblement institutionnalisé. Lorsqu'il s'agit de mesurer une telle forme de capital, on ne peut donc se contenter d'indicateurs renvoyant à des possessions instituées (titres, diplômes, compétences ou objets); il faut également en repérer les traces non institutionnalisées qui se manifestent notamment par des pratiques ${ }^{128}$. C'est précisément ce que nous proposons au travers d'une troisième dimension, la dimension manifestée. Les indicateurs retenus sont les différents types de congrès auxquels les infirmières sont susceptibles d'assister ${ }^{129}$, l'affiliation à l'ASI, l'orientation de patients vers des spécialistes de médecine complémentaire ${ }^{130}$ et l'administration de soins allant à l'encontre des consignes de l'employeur ou de la

\footnotetext{
${ }^{128}$ Décrivant le «capital clinique» en voie de constitution au sein du champ médical au XIX ${ }^{\mathrm{e}}$ siècle, Patrice PInell (2009) privilégie également des indicateurs relatifs à des pratiques. Les médecins fortement dotés en capital clinique sont ceux qui se distinguent par des pratiques de production académique: "enrichissement de la sémiologie, caractérisation d'une nouvelle entité pathologique, élaboration d'une nouvelle classification, publication d'ouvrages de synthèse, description d'une nouvelle méthode opératoire» (p. 329).

${ }^{129}$ Il s'agit des congrès sur les soins infirmiers, sur le développement personnel et sur les médecines complémentaires. Il était demandé aux infirmières d'indiquer si elles avaient assisté à de tels congrès au cours des 12 mois précédant l'enquête.

${ }^{130} \mathrm{La}$ question était: "Vous arrive-t-il de conseiller à un patient d'avoir recours à de la médecine complémentaire (homéopathie, acupuncture, chiropratique, etc.) ?» Les modalités de réponse étaient «jamais», "parfois",
} 
hiérarchie $^{131}$. Ces pratiques ont en commun de manifester une spécificité professionnelle distincte de l'orthodoxie médicale et des injonctions managériales. Alors que certaines (assister à des congrès de soins infirmiers, être affiliée à l'ASI) sont légitimes, car issues d'une lutte pour la reconnaissance professionnelle, d'autres (administrer des soins à l'encontre des consignes de son employeur) sont illégitimes, car leur reconnaissance n'est pas encore acquise. Si ces pratiques dissimulées semblent a priori peu compatibles avec l'idée d'un capital censé offrir des avantages à leurs possédants, il faut toutefois rappeler que, tel que nous le définissons, le capital infirmier est en voie de constitution, et ne fait donc pas tant l'enjeu d'une lutte de possession que d'une lutte de reconnaissance. Bien qu'elle puisse sans doute tendre à l'occasion vers une forme de "capital militant» (Matonti \& Poupeau, 2004) et mener à des formes explicites de contestation ${ }^{132}$, la dimension manifestée du capital infirmier ne s'y réduit en aucun cas. Elle sous-tend plus sûrement des pratiques hétérodoxes individuelles et dissimulées, ou des mobilisations consensuelles qui, typiques des soins infirmiers, se définissent comme des actions sans adversaire explicite et reposant sur "l'agencement d'une morale pratique (le care) avec des contextes de travail locaux, autour du thème fédérateur, plus

"souvent", «tous les jours ou presque». Pour l'analyse, les deux dernières modalités ont été regroupées sous l'appellation "souvent».

${ }^{131}$ La question était: "Vous arrive-t-il de proposer à vos patients des soins qui ne respectent pas les consignes de votre employeur et/ou hiérarchie (médicaments homéopathiques, guérisseurs ou faiseurs de secrets, etc.) ?» Les modalités de réponse étaient "jamais", "parfois", "souvent», "tous les jours ou presque». Ces modalités ont été regroupées en deux modalités, la modalité «jamais» (recodée en "non») d'une part, les trois autres modalités (recodées en "oui») d'autre part. Bien qu'il s'agisse d'une question potentiellement sensible, pas moins de $33 \%$ des infirmières ont répondu par l'affirmative.

${ }^{132}$ On sait que les formes collectives de contestation sont rares au sein de la profession. Ce qu'Ivan Sainsaulieu (2012) explique par les contraintes logistiques (obligation de continuité de service), morales (relation au patient) et familiales (prise en charge des enfants) ainsi que par la socialisation historiquement genrée des infirmières qui "ne les prédispose pas à la contestation politique» (p. 475). 
ou moins explicite, de "l'amélioration de la qualité des soins"” (SAinsaulieu, 2012, p. 486-487).

À ces trois dimensions du capital médical et du capital infirmier, nous avons ajouté le taux d'activité ${ }^{133}$. Notre enquête exploratoire nous a en effet permis de constater que, dans une profession fortement féminisée où le temps partiel constitue la norme ${ }^{134}$, celles qui exercent à un taux d'activité élevé disposent d'avantages décisifs, qu'il s'agisse de la possibilité de suivre les formations les plus longues ou de l'accès aux postes à responsabilité. Le taux d'activité élevé peut ainsi être considéré comme une ressource qui favorise l'accumulation de capital.

\section{Annexe 3. Tableaux}

Tous les tableaux suivants reposent sur les données de l'EPRIS.

Tableau A1. Secteurs, cadre et taux d'occupation selon le sexe (\%)

\begin{tabular}{|l|l|c|c|c|r|}
\cline { 3 - 6 } \multicolumn{2}{c|}{} & Femme & Homme & Total & \multicolumn{1}{c|}{$\mathbf{n}$} \\
\hline Ensemble & & 84,3 & 15,7 & 100 & 2913 \\
\cline { 2 - 6 } Secteurs & Anesthésie & 41,2 & 58,8 & 100 & 34 \\
\cline { 2 - 6 } & Bloc & 70,9 & 29,1 & 100 & 86 \\
\cline { 2 - 6 } & Cabinet & 93,9 & 6,1 & 100 & 33 \\
\cline { 2 - 6 } & Chirurgie & 84,7 & 15,3 & 100 & 242 \\
\cline { 2 - 6 } & Direction & 68,2 & 31,8 & 100 & 22 \\
\cline { 2 - 6 } & Santé scolaire & 95,6 & 4,4 & 100 & 135 \\
\cline { 2 - 6 } & EMS & 87,9 & 12,1 & 100 & 157 \\
\cline { 2 - 6 } & Enseignement & 83,3 & 16,7 & 100 & 12 \\
\hline
\end{tabular}

${ }^{133}$ Cette variable a été codée en trois modalités: 0-60\%, 61-80\% et $81-100 \%$.

${ }^{134}$ Dans notre échantillon, $65 \%$ des infirmières travaillent à temps partiel. 


\begin{tabular}{|c|c|c|c|c|c|}
\hline & Femme & Homme & Total & $\mathbf{n}$ \\
\hline & Entreprise & 93,9 & 6,1 & 100 & 49 \\
\hline & Équipe mobile & 93,3 & 6,7 & 100 & 15 \\
\hline & ES, HES & 83,6 & 16,4 & 100 & 116 \\
\hline & \begin{tabular}{|l} 
Gériatrie \\
hospitalière
\end{tabular} & 89,2 & 10,8 & 100 & 65 \\
\hline & Gynécologie & 94,9 & 5,1 & 100 & 39 \\
\hline & Indépendantes & 92,6 & 7,4 & 100 & 27 \\
\hline & Médecine & 83,6 & 16,4 & 100 & 171 \\
\hline & Obstétrique & 97,1 & 2,9 & 100 & 34 \\
\hline & Oncologie & 94,5 & 5,5 & 100 & 55 \\
\hline & Pédiatrie & 93,8 & 6,2 & 100 & 273 \\
\hline & Pédopsychiatrie & 81,3 & 18,8 & 100 & 16 \\
\hline & Pool & 96,6 & 3,4 & 100 & 29 \\
\hline & Psychiatrie & 66,4 & 33,6 & 100 & 229 \\
\hline & Psychogériatrie & 86,4 & 13,6 & 100 & 44 \\
\hline & Rééducation & 82,6 & 17,4 & 100 & 115 \\
\hline & Salle de réveil & 84,4 & 15,6 & 100 & 32 \\
\hline & \begin{tabular}{|l} 
Service \\
ambulatoire
\end{tabular} & 83,9 & 16,1 & 100 & 93 \\
\hline & Socio-éducatif & 82,5 & 17,5 & 100 & 63 \\
\hline & Soins continus & 85,7 & 14,3 & 100 & 70 \\
\hline & Soins à domicile & 93,2 & 6,8 & 100 & 176 \\
\hline & Soins intensifs & 81,3 & 18,7 & 100 & 166 \\
\hline & Soins palliatifs & 87,5 & 12,5 & 100 & 40 \\
\hline & $\begin{array}{l}\text { Spécialités } \\
\text { médicales }\end{array}$ & 81,6 & 18,4 & 100 & 136 \\
\hline & Urgences & 74,1 & 25,9 & 100 & 139 \\
\hline Cadre & Non & 86,3 & 13,7 & 100 & 2362 \\
\hline & Oui & 74,1 & 25,9 & 100 & 494 \\
\hline $\begin{array}{l}\text { Taux } \\
\text { d'occupation }\end{array}$ & Moyenne & 78,1 & 92,6 & 80,4 & 2904 \\
\hline & Écart-type & 19,4 & 12,9 & 19,2 & \\
\hline
\end{tabular}


Tableau A2. Quelques caractéristiques sociodémographiques des infirmières

\begin{tabular}{|c|c|c|c|}
\hline Variables & Modalités & $\mathbf{N}$ & Pourcentage \\
\hline \multirow[t]{6}{*}{$\begin{array}{l}\text { Formation } \\
\text { la plus élevée }\end{array}$} & $\begin{array}{l}\text { Diplôme de base (équ. } \\
\text { HES ou ES) }\end{array}$ & 527 & 18,6 \\
\hline & Postgrade court & 534 & 18,8 \\
\hline & Postgrade long & 1585 & 55,9 \\
\hline & Postgrade Bachelor & 144 & 5,1 \\
\hline & Master & 44 & 1,6 \\
\hline & $\mathrm{PhD}$ & 3 & 0,1 \\
\hline \multirow[t]{2}{*}{ Genre } & Femme & 2455 & 84,3 \\
\hline & Homme & 458 & 15,7 \\
\hline \multirow[t]{2}{*}{ Cadre } & Oui & 497 & 17,3 \\
\hline & Non & 2369 & 82,7 \\
\hline \multirow[t]{2}{*}{ Nationalité } & Suisse & 1766 & 61,7 \\
\hline & Étranger & 1094 & 38,3 \\
\hline \multirow[t]{13}{*}{ Fonction } & Cadre supérieur & 82 & 2,8 \\
\hline & Clinicienne & 75 & 2,6 \\
\hline & Consultante & 17 & 0,6 \\
\hline & Diplômée & 1559 & 53,3 \\
\hline & Enseignante chercheuse & 122 & 4,2 \\
\hline & Enseignement de terrain & 18 & 0,6 \\
\hline & ICLS & 25 & 0,9 \\
\hline & ICS & 66 & 2,3 \\
\hline & ICUS & 267 & 9,1 \\
\hline & ISC & 49 & 1,7 \\
\hline & Liaison & 8 & 0,3 \\
\hline & Recherche & 35 & 1,2 \\
\hline & Spécialisée & 600 & 20,5 \\
\hline
\end{tabular}




\begin{tabular}{|c|c|c|c|}
\hline Variables & Modalités & $\mathbf{N}$ & Pourcentage \\
\hline \multirow[t]{8}{*}{$\begin{array}{l}\text { Type } \\
\text { d'établissement }\end{array}$} & $\begin{array}{l}\text { Hôpital universitaire } \\
\text { ou cantonal }\end{array}$ & 1148 & 39,7 \\
\hline & Santé scolaire, entreprise & 162 & 5,6 \\
\hline & EMS & 206 & 7,1 \\
\hline & $\begin{array}{l}\text { Enseignement } \\
\text { et recherche }\end{array}$ & 98 & 3,4 \\
\hline & $\begin{array}{l}\text { Hôpital de zone } \\
\text { ou régional }\end{array}$ & 619 & 21,4 \\
\hline & Privé & 193 & 6,7 \\
\hline & Psy/Social & 199 & 6,9 \\
\hline & Soins à domicile & 268 & 9,3 \\
\hline \multirow[t]{15}{*}{ Secteurs } & Anesthésie & 34 & 1,2 \\
\hline & Bloc opératoire & 86 & 2,9 \\
\hline & Cabinet & 33 & 1,1 \\
\hline & Chirurgie & 243 & 8,3 \\
\hline & Direction & 22 & 0,8 \\
\hline & Santé scolaire & 135 & 4,6 \\
\hline & EMS & 160 & 5,5 \\
\hline & $\begin{array}{l}\text { Enseignement } \\
\text { sur le terrain }\end{array}$ & 12 & 0,4 \\
\hline & Entreprise & 49 & 1,7 \\
\hline & Équipe mobile & 15 & 0,5 \\
\hline & ES, HES & 116 & 4,0 \\
\hline & Gériatrie hospitalière & 65 & 2,2 \\
\hline & Gynécologie & 40 & 1,4 \\
\hline & Indépendantes & 27 & 0,9 \\
\hline & Médecine & 171 & 5,9 \\
\hline
\end{tabular}




\begin{tabular}{|c|c|c|c|}
\hline Variables & Modalités & $\mathbf{N}$ & Pourcentage \\
\hline & Obstétrique & 34 & 1,2 \\
\hline & Oncologie & 56 & 1,9 \\
\hline & Pédiatrie & 273 & 9,3 \\
\hline & Pédopsychiatrie & 16 & 0,5 \\
\hline & Pool & 29 & 1,0 \\
\hline & Psychiatrie & 229 & 7,8 \\
\hline & Psychogériatrie & 44 & 1,5 \\
\hline & Rééducation & 116 & 4,0 \\
\hline & Salle de réveil & 32 & 1,1 \\
\hline & Service ambulatoire & 93 & 3,2 \\
\hline & Socio-éducatif & 63 & 2,2 \\
\hline & Soins continus & 70 & 2,4 \\
\hline & Soins à domicile & 176 & 6,0 \\
\hline & Soins intensifs & 167 & 5,7 \\
\hline & Soins palliatifs & 40 & 1,4 \\
\hline & Spécialités médicales & 136 & 4,7 \\
\hline & Urgences & 141 & 4,8 \\
\hline Âge & Moyenne (écart-type) & 2897 & $42,8(10,3)$ \\
\hline Ensemble & & 2923 & \\
\hline
\end{tabular}


Tableau A3. Distribution des infirmières de nationalité étrangère selon le canton, le secteur d'activité, le sexe et l'âge (\%).

\begin{tabular}{|c|c|c|c|c|}
\hline Variables & Modalités & $\begin{array}{c}\text { Infirmières } \\
\text { étrangères } \\
n=1094 \\
(38,3 \%)\end{array}$ & $\begin{array}{c}\text { Infirmières } \\
\text { suisses } \\
n=1766 \\
(61,7 \%)\end{array}$ & Ensemble \\
\hline \multirow[t]{7}{*}{ Canton } & Genève & 58,5 & 41,5 & 25,0 \\
\hline & Neuchâtel & 39,7 & 60,3 & 7,4 \\
\hline & Jura & 36,6 & 63,4 & 3,3 \\
\hline & Vaud & 32,3 & 67,7 & 47,6 \\
\hline & Valais & 27,8 & 72,2 & 7,0 \\
\hline & Fribourg & 24,0 & 76,0 & 6,9 \\
\hline & Berne & 19,8 & 80,2 & 2,9 \\
\hline \multirow{17}{*}{$\begin{array}{l}\text { Secteur } \\
\text { d'activité }\end{array}$} & Anesthésie & 57,6 & 42,4 & 1,2 \\
\hline & Bloc opératoire & 64,7 & 35,3 & 3,0 \\
\hline & Cabinet & 21,2 & 78,8 & 1,2 \\
\hline & Chirurgie & 42,3 & 57,7 & 8,2 \\
\hline & Direction & 57,1 & 42,9 & 0,7 \\
\hline & Santé scolaire & 7,5 & 92,5 & 4,7 \\
\hline & EMS & 34,4 & 65,6 & 5,4 \\
\hline & $\begin{array}{l}\text { Enseignement } \\
\text { sur le terrain }\end{array}$ & 18,2 & 81,8 & 0,4 \\
\hline & Entreprise & 27,1 & 72,9 & 1,7 \\
\hline & Équipe mobile & 40,0 & 60,0 & 0,5 \\
\hline & ES, HES & 21,1 & 78,9 & 4,0 \\
\hline & Gériatrie hospitalière & 64,6 & 35,4 & 2,3 \\
\hline & Gynécologie & 55,3 & 44,7 & 1,3 \\
\hline & Indépendantes & 14,8 & 85,2 & 0,9 \\
\hline & Médecine & 36,3 & 63,7 & 5,9 \\
\hline & Obstétrique & 44,1 & 55,9 & 1,2 \\
\hline & Oncologie & 34,5 & 65,5 & 1,9 \\
\hline
\end{tabular}




\begin{tabular}{|c|c|c|c|c|}
\hline Variables & Modalités & $\begin{array}{c}\text { Infirmières } \\
\text { étrangères } \\
n=1094 \\
(38,3 \%)\end{array}$ & $\begin{array}{c}\text { Infirmières } \\
\text { suisses } \\
n=1766 \\
(61,7 \%) \\
\end{array}$ & Ensemble \\
\hline & Pédiatrie & 34,5 & 65,5 & 9,3 \\
\hline & Pédopsychiatrie & 43,8 & 56,3 & 0,6 \\
\hline & Pool & 37,9 & 62,1 & 1,0 \\
\hline & Psychiatrie & 38,8 & 61,2 & 7,9 \\
\hline & Psychogériatrie & 43,8 & 56,3 & 0,6 \\
\hline & Rééducation & 46,8 & 53,2 & 3,9 \\
\hline & Salle de réveil & 45,2 & 54,8 & 1,1 \\
\hline & Service ambulatoire & 36,7 & 63,3 & 3,1 \\
\hline & Socio-éducatif & 6,6 & 93,4 & 2,1 \\
\hline & Soins continus & 40,0 & 60,0 & 2,4 \\
\hline & Soins à domicile & 24,1 & 75,9 & 6,1 \\
\hline & Soins intensifs & 57,7 & 42,3 & 5,7 \\
\hline & Soins palliatifs & 33,3 & 66,7 & 1,4 \\
\hline & Spécialités médicales & 49,3 & 50,7 & 4,7 \\
\hline & Urgences & 48,9 & 51,1 & 4,8 \\
\hline \multirow[t]{2}{*}{ Sexe } & Homme & 48,8 & 51,2 & 15,6 \\
\hline & Femme & 36,3 & 63,7 & 84,4 \\
\hline \multirow[t]{5}{*}{ Âge } & $<30$ ans & 42,2 & 57,8 & 12,1 \\
\hline & $30-39$ ans & 46,4 & 53,6 & 27,2 \\
\hline & $40-49$ ans & 41,3 & 58,7 & 30,5 \\
\hline & $50-59$ ans & 28,9 & 71,1 & 25,8 \\
\hline & 60 ans et plus & 12,8 & 87,2 & 4,4 \\
\hline
\end{tabular}


Tableau A4. Revenu équivalent plein temps (CHF) selon les caractéristiques sociodémographiques et socioprofessionnelles

\begin{tabular}{|c|c|c|c|}
\hline & Moyenne & $\mathbf{N}$ & Écart-type \\
\hline Ensemble & 6434 & 2420 & 1423 \\
\hline \multicolumn{4}{|l|}{ Fractions } \\
\hline Élites infirmières & 7035 & 556 & 1573 \\
\hline Hétérodoxes & 6743 & 562 & 1515 \\
\hline Dominées médicales & 6029 & 754 & 1181 \\
\hline Dominantes médicales & 6065 & 548 & 1160 \\
\hline \multicolumn{4}{|l|}{ Statut professionnel } \\
\hline Non-cadre & 6207 & 1946 & 1282 \\
\hline Cadre & 7455 & 430 & 1552 \\
\hline \multicolumn{4}{|c|}{ Lieu d'obtention du diplôme } \\
\hline En Suisse & 6525 & 1369 & 1479 \\
\hline À l'étranger & 6310 & 1030 & 1341 \\
\hline \multicolumn{4}{|l|}{ Sexe } \\
\hline Femme & 6404 & 2017 & 1349 \\
\hline Homme & 6573 & 394 & 1743 \\
\hline \multicolumn{4}{|l|}{ Nationalité } \\
\hline Suisse & 6614 & 1496 & 1483 \\
\hline Étrangère & 6133 & 882 & 1252 \\
\hline \multicolumn{4}{|l|}{ Lieu de résidence } \\
\hline Réside en Suisse & 6518 & 1773 & 1499 \\
\hline Réside en France & 6206 & 630 & 1142 \\
\hline \multicolumn{4}{|l|}{ Canton de travail } \\
\hline Vaud & 6287 & 1159 & 1441 \\
\hline Berne & 6140 & 70 & 1548 \\
\hline Fribourg & 7012 & 169 & 1535 \\
\hline Genève & 6682 & 577 & 1287 \\
\hline
\end{tabular}




\begin{tabular}{|c|c|c|c|}
\hline & Moyenne & $\mathbf{N}$ & Écart-type \\
\hline Jura & 6354 & 83 & 1197 \\
\hline Neuchâtel & 6415 & 180 & 1335 \\
\hline Valais & 6210 & 168 & 1491 \\
\hline \multicolumn{4}{|c|}{ Formation après le diplôme de base } \\
\hline Non & 5721 & 419 & 1140 \\
\hline Oui & 6581 & 1965 & 1430 \\
\hline \multicolumn{4}{|l|}{ Type d'établissement } \\
\hline Hôpital universitaire cantonal & 6427 & 954 & 1206 \\
\hline Santé scolaire, Entreprises & 6730 & 131 & 1154 \\
\hline EMS & 6655 & 183 & 1611 \\
\hline Enseignement, recherche & 8659 & 75 & 1626 \\
\hline $\begin{array}{l}\text { Hôpital de zone } \\
\text { ou régional }\end{array}$ & 6106 & 516 & 1291 \\
\hline Cabinet médical, clinique & 5791 & 150 & 1446 \\
\hline $\begin{array}{l}\text { Établ. psychiatrique, socio- } \\
\text { éduc., ou carcéral }\end{array}$ & 6511 & 168 & 1514 \\
\hline Soins à domicile & 6517 & 220 & 1616 \\
\hline \multicolumn{4}{|l|}{ Ancienneté } \\
\hline $\begin{array}{l}\text { Nombre d'années depuis } \\
\text { l'obtention du diplôme }\end{array}$ & $73^{a}$ & 2398 & $2^{\mathrm{b}}$ \\
\hline
\end{tabular}

Note: Toutes les relations bivariées sont significatives avec une valeur $p<0,001$, à l'exception du sexe avec une valeur $p=0,031$. 
Tableau A5. Modèle explicatif du revenu équivalent plein temps

\begin{tabular}{|l|c|}
\cline { 2 - 2 } \multicolumn{1}{c|}{} & Coefficients \\
\hline Constante & $5088^{* * *}$ \\
\hline Cadres & $867^{* * *}$ \\
\hline Diplôme obtenu à l'étranger & $-226^{* *}$ \\
\hline Nombre d'années depuis l'obtention du diplôme & $66^{* * *}$ \\
\hline Homme & $311^{* * *}$ \\
\hline Étranger & -109 \\
\hline Résidence en France & $-177^{*}$ \\
\hline Canton de travail: BE & -156 \\
\hline Canton de travail: FR & $332^{* * *}$ \\
\hline Canton de travail: GE & $366^{* * *}$ \\
\hline Canton de travail: JU & -22 \\
\hline Canton de travail: NE & 31 \\
\hline Canton de travail: VS & -33 \\
\hline Formation après diplôme & $209^{* * *}$ \\
\hline Santé scolaire, entreprise & $-285^{* *}$ \\
\hline EMS & $-239^{*}$ \\
\hline Enseignement, recherche & $1454^{* * *}$ \\
\hline Hôpital de zone ou régional & $-293^{* * *}$ \\
\hline Cabinet médical, clinique & $-674^{* * *}$ \\
\hline Établ. psychiatrique, socio-éducatif ou carcéral & $-252^{* *}$ \\
\hline Soins à domicile & $-442^{* * *}$ \\
\hline N & 2228 \\
\hline R² & 0,477 \\
\hline$R^{2}$ ajusté & 0,472 \\
\hline
\end{tabular}

Notes: ${ }^{* * *} p<0,001 ;{ }^{* *} p<0,01 ;{ }^{*} p<0,05$. Modèle de régression linéaire. Les catégories de références sont: non-cadre, diplôme obtenu en Suisse, femme, nationalité suisse, résidence en Suisse, canton de Vaud, hôpital universitaire ou cantonal. 
Tableau A6. Le prestige des secteurs selon certaines caractéristiques classiques

\begin{tabular}{|c|c|}
\hline & Coefficients \\
\hline Constante & $-3,98$ \\
\hline \multicolumn{2}{|l|}{ Stabilité de la situation contractuelle } \\
\hline Ancienneté moyenne & 0,13 \\
\hline Taux d'emploi secteur public & 0,38 \\
\hline Revenu moyen & 0,00 \\
\hline \multicolumn{2}{|l|}{ Qualifications personnelles } \\
\hline Formation & 3,23 \\
\hline Âge moyen & $-0,03$ \\
\hline \multicolumn{2}{|l|}{ Autonomie } \\
\hline Part des tâches médico-déléguées & $2,08 * * *$ \\
\hline \multicolumn{2}{|l|}{ Autorité } \\
\hline Taux de cadre & $-5,15 *$ \\
\hline $\mathrm{N}$ & 20 \\
\hline $\mathrm{R}^{2}$ & 0.84 \\
\hline $\mathrm{R}^{2}$ ajusté & 0.75 \\
\hline
\end{tabular}

Notes: ${ }^{* *} \mathrm{p}<0,001 ;{ }^{* *} \mathrm{p}<0,01 ;{ }^{*} \mathrm{p}<0,05$. Modèle de régression linéaire. 
Tableau A7. Le prestige des secteurs selon certaines caractéristiques spécifiques

\begin{tabular}{|l|c|c|c|}
\cline { 2 - 4 } \multicolumn{1}{c|}{} & \multicolumn{3}{c|}{ Coefficients } \\
\cline { 2 - 4 } \multicolumn{1}{c|}{} & Modèle 1 & Modèle 2 & Modèle 3 \\
\hline Constante & $21,74^{* * *}$ & $20,52^{* * *}$ & 2,62 \\
\hline Indice soins relationnels & $-2,91^{* * *}$ & $-3,56 * * *$ & $-0,32$ \\
\hline Indice soins confort et hygiène & & 0,36 & $-0,00$ \\
\hline Indice soins médico-techniques & & & $1,05 * *$ \\
\hline $\mathrm{N}$ & 20 & 20 & 20 \\
\hline $\mathrm{R}^{2}$ & 0,52 & 0,59 & 0,77 \\
\hline Valeur $\mathrm{p} \Delta \mathrm{R}^{2}$ & - & 0,106 & 0,003 \\
\hline $\mathrm{R}^{2}$ ajusté & 0,49 & 0,54 & 0,73 \\
\hline RMSE & 0,89 & 0,85 & 0,66 \\
\hline
\end{tabular}

Notes: ${ }^{* *} p<0,001 ;{ }^{* *} p<0,01 ;{ }^{*} p<0,05$. Modèles de régression linéaire. 
Tableau A8. Motivations selon les caractéristiques sociales antécédentes

\begin{tabular}{|c|c|c|c|c|}
\hline & $\begin{array}{l}\text { Motivation } \\
\text { humaniste }\end{array}$ & $\begin{array}{c}\text { Motivation } \\
\text { Antivocationnelle }\end{array}$ & \begin{tabular}{|c|} 
Motivation \\
promotionnelle
\end{tabular} & $\begin{array}{c}\text { Motivation } \\
\text { second choix }\end{array}$ \\
\hline \multicolumn{5}{|c|}{ Origine sociale } \\
\hline $\begin{array}{l}\text { Classes } \\
\text { moyennes }\end{array}$ & -0001 & $0071^{*}$ & -0039 & $0092^{* * *}$ \\
\hline $\begin{array}{l}\text { Classes } \\
\text { supérieures }\end{array}$ & -0026 & $0100^{* *}$ & -0056 & $0135^{* * *}$ \\
\hline \multicolumn{5}{|l|}{ Sexe } \\
\hline Homme & $-0273^{* * *}$ & $0295^{* * *}$ & -0015 & $0226^{* * *}$ \\
\hline \multicolumn{5}{|l|}{ Nationalité } \\
\hline Étranger & -0052 & $0154^{* * *}$ & $0117^{* * *}$ & $0161^{* * *}$ \\
\hline $\mathrm{N}$ & 2587 & 2587 & 2587 & 2587 \\
\hline $\mathrm{R}^{2}$ & 0,012 & 0,031 & 0,007 & 0,042 \\
\hline $\mathrm{R}^{2}$ ajusté & 0,010 & 0,030 & 0,006 & 0,040 \\
\hline
\end{tabular}

Notes: ${ }^{* * *} p<0,001 ;{ }^{* *} p<0,01 ;{ }^{*} p<0,05$. Modèles de régression linéaire. Les catégories de références sont: classes populaires, femme, Suisse. 
Tableau A9. Appartenance aux 4 fractions selon les types de motivations

\begin{tabular}{|c|c|c|c|}
\hline & $\begin{array}{c}\text { Élites infirmières } \\
\text { vs } \\
\text { Dominées } \\
\text { médicales }\end{array}$ & \begin{tabular}{|c|} 
Hétérodoxes \\
vs \\
Dominées \\
médicales
\end{tabular} & $\begin{array}{c}\text { Dominantes médicales } \\
\text { vs } \\
\text { Dominées médicales }\end{array}$ \\
\hline \multicolumn{4}{|l|}{ Motivations } \\
\hline $\begin{array}{l}\text { Motivation } \\
\text { Humaniste }\end{array}$ & $-0,043$ & 0,053 & $-0,083$ \\
\hline $\begin{array}{l}\text { Motivation } \\
\text { Antivocationnelle }\end{array}$ & $0,224^{* * *}$ & $0,183^{* *}$ & $0,187^{* *}$ \\
\hline $\begin{array}{l}\text { Motivation } \\
\text { Promotionnelle }\end{array}$ & $0,374^{* * *}$ & $-0,035$ & $0,354^{* * *}$ \\
\hline $\begin{array}{l}\text { Motivation } \\
\text { Second choix }\end{array}$ & 0,145 & $-0,008$ & 0,071 \\
\hline \multicolumn{4}{|l|}{ Origine sociale } \\
\hline $\begin{array}{l}\text { Classes } \\
\text { moyennes }\end{array}$ & 0,017 & $0,245^{*}$ & 0,190 \\
\hline $\begin{array}{l}\text { Classes } \\
\text { supérieures }\end{array}$ & $-0,057$ & 0,197 & 0,156 \\
\hline \multicolumn{4}{|l|}{ Sexe } \\
\hline Homme & $0,423^{* * *}$ & $-0,156$ & $0,539 * * *$ \\
\hline \multicolumn{4}{|l|}{ Nationalité } \\
\hline Étranger & $-0,165$ & $-0,770 * * *$ & $0,204^{*}$ \\
\hline Âge & $0,057^{* * *}$ & $0,065^{* * *}$ & $-0,020^{* * *}$ \\
\hline $\mathrm{N}$ & \multicolumn{3}{|l|}{2574} \\
\hline $\begin{array}{l}\text { Pseudo-R } \\
\text { de Nagelkerke }\end{array}$ & \multicolumn{3}{|l|}{0,183} \\
\hline
\end{tabular}

Notes: ${ }^{* * *} p<0,001 ;{ }^{* *} p<0,01 ;{ }^{*} p<0,05$. Modèle de régression logistique multinomiale. Les catégories de références sont: classes populaires, femme, nationalité suisse. 
Tableau A10. Rapport «désenchanté» à la profession selon le degré de religiosité, le genre et l'origine sociale

\begin{tabular}{|l|c|}
\cline { 2 - 2 } \multicolumn{1}{l}{} & \multicolumn{1}{c|}{ Coefficients } \\
\hline Degré religiosité/spiritualité & $-0,119^{* * *}$ \\
\hline Sexe & $1,025^{* * *}$ \\
\hline Homme & $0,260^{* *}$ \\
\hline Origine sociale & $0,504^{* * *}$ \\
\hline Classes moyennes & 0,075 \\
\hline Classes supérieures & $-0,113$ \\
\hline Fractions & $-0,122$ \\
\hline Hétérodoxes & \multicolumn{2}{|c|}{} \\
\hline Dominées médicales & 0,087 \\
\hline Dominantes médicales & 0,143 \\
\hline Âge & 0,066 \\
\hline $36-49$ ans & $-0,049$ \\
\hline 50 ans et plus & 2374 \\
\hline Nationalité & \\
\hline Étranger & \\
\hline N & \\
\hline Pseudo-R ${ }^{*}$ de Nagelkerke & \\
\hline
\end{tabular}

Notes: ${ }^{* *} p<0,001 ;{ }^{* *} p<0,01 ;{ }^{*} p<0,05$. Modèle de régression logistique binomiale. Les catégories de références sont: classes populaires, femme, élites infirmières, nationalité suisse, moins de 36 ans. 


\section{Annexe 4. L'espace infirmier}

Le graphique et les tableaux suivants reposent sur les données de l'EPRIS.

Graphique A1. L'espace infirmier (modalités actives relatives aux capitaux médical et infirmier dans le plan factoriel 1-2)

Axe 2 - Taux modifiés : 0.23

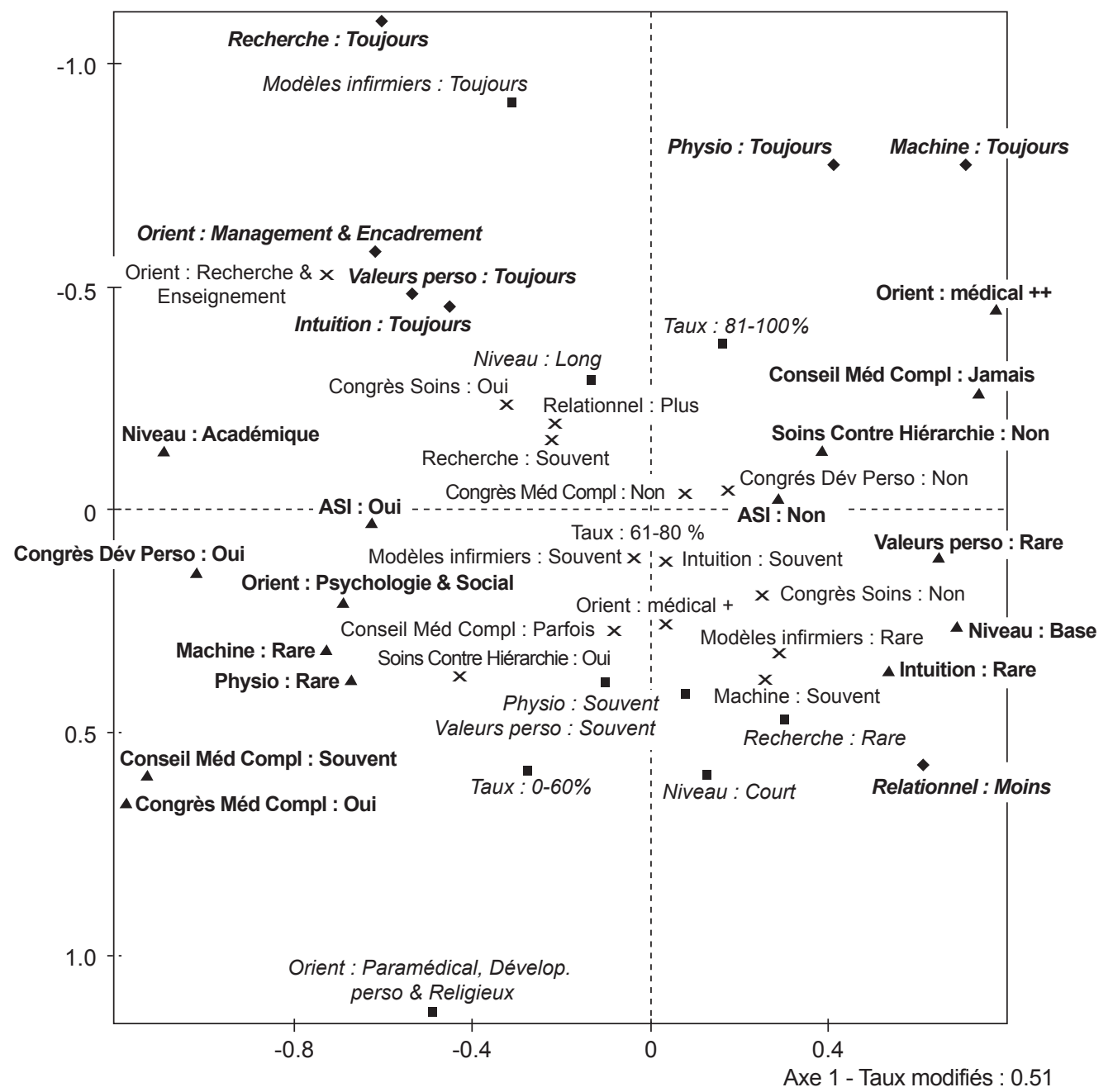

Source: EPRIS.

Note: $\mathrm{N}=2$ 923. Les modalités en gras (triangles) sont celles retenues pour l'interprétation du premier axe. Les modalités en italique (carrés) sont celles retenues pour l'interprétation du deuxième axe. Les modalités en gras italique (losanges) sont celles retenues pour l'interprétation des deux axes. Les modalités en petite police (croix) sont celles dont la contribution est inférieure à la contribution moyenne et qui n'ont donc pas été retenues pour l'interprétation des axes.

En gras: variables et modalités dont la contribution est égale ou supérieure à la moyenne (pour les variables: $100 / 16=6,25$; pour les modalités: $100 / 46=2,17$ ). 
Tableau A11. Contributions (en \%) des variables et modalités actives de l'ACM

\begin{tabular}{|c|c|c|c|c|c|}
\hline & Variable & Modalité & Axe 1 & Axe 2 & $\mathbf{N}$ \\
\hline \multirow{9}{*}{ 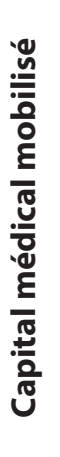 } & \multirow{4}{*}{$\begin{array}{l}\text { Mobilisation } \\
\text { des connaissances en } \\
\text { utilisation de machines/ } \\
\text { appareils techniques }\end{array}$} & Rare Machine & 6,8 & 1,7 & 1276 \\
\hline & & Souvent Machine & 0,8 & 1,6 & 831 \\
\hline & & Toujours Machine & 5,8 & 8,5 & 801 \\
\hline & & Total & 13,4 & 11,8 & 2908 \\
\hline & \multirow{4}{*}{\begin{tabular}{|l} 
Mobilisation \\
des connaissances \\
en physiopathologie
\end{tabular}} & Rare Physio & 2,1 & 1,5 & 673 \\
\hline & & Souvent Physio & 0,1 & 2,5 & 1361 \\
\hline & & Toujours Physio & 2,5 & 9,4 & 877 \\
\hline & & Total & 4,7 & 13,4 & 2911 \\
\hline & Total & & 18,1 & 25,2 & -- \\
\hline \multirow{20}{*}{ 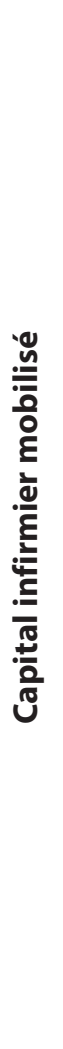 } & \multirow{4}{*}{$\begin{array}{l}\text { Mobilisation } \\
\text { des connaissances } \\
\text { en recherche }\end{array}$} & Rare Recherche & 2,0 & 4,2 & 1518 \\
\hline & & Souvent Recherche & 0,7 & 0,4 & 989 \\
\hline & & Toujours Recherche & 2,1 & 8,7 & 408 \\
\hline & & Total & 4,9 & 13,3 & 2915 \\
\hline & \multirow{4}{*}{$\begin{array}{l}\text { Mobilisation des valeurs } \\
\text { personnelles }\end{array}$} & Rare Valeurs perso & 4,0 & 0,2 & 656 \\
\hline & & Souvent Valeurs perso & 0,2 & 2,7 & 1193 \\
\hline & & $\begin{array}{l}\text { Toujours Valeurs } \\
\text { perso }\end{array}$ & 4,4 & 4,5 & 1057 \\
\hline & & Total & 8,6 & 7,3 & 2906 \\
\hline & \multirow{4}{*}{$\begin{array}{l}\text { Mobilisation } \\
\text { des connaissances } \\
\text { en modèles théoriques } \\
\text { infirmiers }\end{array}$} & Rare Modèles Inf & 1,5 & 1,9 & 1256 \\
\hline & & Souvent Modèles Inf & 0,5 & 0,2 & 1128 \\
\hline & & Toujours Modèles Inf & 0,7 & 7,6 & 517 \\
\hline & & Total & 2,8 & 9,7 & 2901 \\
\hline & \multirow{3}{*}{$\begin{array}{l}\text { Mobilisation } \\
\text { des compétences } \\
\text { relationnelles }\end{array}$} & Moins Relationnel & 4,1 & 3,5 & 753 \\
\hline & & Plus Relationnel & 1,4 & 1,2 & 2167 \\
\hline & & Total & 5,5 & 4,7 & 2920 \\
\hline & \multirow{4}{*}{$\begin{array}{l}\text { Mobilisation } \\
\text { de l'intuition }\end{array}$} & Rare Intuition & 2,1 & 1,2 & 630 \\
\hline & & Souvent Intuition & 0,1 & 0,4 & 1422 \\
\hline & & Toujours Intuition & 2,5 & 3,1 & 858 \\
\hline & & Total & 4,7 & 4,7 & 2910 \\
\hline & Total & & 26,5 & 39,7 & -- \\
\hline
\end{tabular}




\begin{tabular}{|c|c|c|c|c|c|}
\hline & Variable & Modalité & Axe 1 & Axe 2 & $\mathbf{N}$ \\
\hline \multirow{20}{*}{ 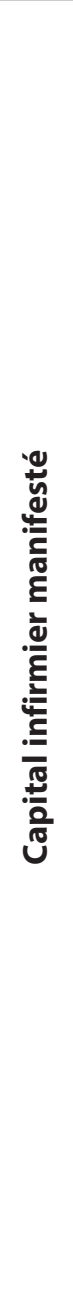 } & \multirow{4}{*}{$\begin{array}{l}\text { Conseiller aux patients } \\
\text { d'avoir recours } \\
\text { à de la médecine } \\
\text { complémentaire }\end{array}$} & $\begin{array}{l}\text { Jamais } \\
\text { ConseilMédCompl }\end{array}$ & 7,3 & 1,1 & 929 \\
\hline & & $\begin{array}{l}\text { Parfois } \\
\text { ConseilMédCompl }\end{array}$ & 0,2 & 1,5 & 1348 \\
\hline & & $\begin{array}{l}\text { Souvent } \\
\text { ConseilMédCompl }\end{array}$ & 3,4 & 1,2 & 184 \\
\hline & & Total & 10,9 & 3,7 & 2461 \\
\hline & \multirow[t]{3}{*}{ Affiliation à I'ASI } & ASI: Non & 2,3 & 0,0 & 1950 \\
\hline & & ASI: Oui & 4,6 & 0,0 & 953 \\
\hline & & Total & 6,9 & 0,0 & 2923 \\
\hline & \multirow{3}{*}{$\begin{array}{l}\text { Congrès } \\
\text { de développement } \\
\text { personnel }\end{array}$} & Non CongrèsDévPerso & 0,8 & 0,0 & 2530 \\
\hline & & Oui CongrèsDévPerso & 5,4 & 0,2 & 393 \\
\hline & & Total & 6,2 & 0,2 & 2923 \\
\hline & \multirow[t]{3}{*}{$\begin{array}{l}\text { Congrès de médecine } \\
\text { complémentaire }\end{array}$} & $\begin{array}{l}\text { Non } \\
\text { CongrèsMédComp }\end{array}$ & 0,2 & 0,1 & 2764 \\
\hline & & $\begin{array}{l}\text { Oui } \\
\text { CongrèsMédComp }\end{array}$ & 3,2 & 1,2 & 159 \\
\hline & & Total & 3,4 & 1,3 & 2923 \\
\hline & \multirow[t]{3}{*}{ Congrès de soins } & Non CongrèsSoins & 1,0 & 0,8 & 1725 \\
\hline & & Oui CongrèsSoins & 1,4 & 1,2 & 1198 \\
\hline & & Total & 2,4 & 2,0 & 2923 \\
\hline & \multirow{3}{*}{$\begin{array}{l}\text { Proposer aux patients } \\
\text { des soins qui ne } \\
\text { respectent pas } \\
\text { les consignes } \\
\text { de l'employeur } \\
\text { et/ou hiérarchie }\end{array}$} & $\begin{array}{l}\text { Non } \\
\text { SoinsContreHiérarchie }\end{array}$ & 3,6 & 0,1 & 1654 \\
\hline & & $\begin{array}{l}\text { Oui } \\
\text { SoinsContreHiérarchie }\end{array}$ & 1,7 & 1,8 & 816 \\
\hline & & Total & 5,2 & 1,9 & 2470 \\
\hline & Total & & 35,0 & 9,1 & -- \\
\hline
\end{tabular}




\begin{tabular}{|c|c|c|c|c|c|}
\hline & Variable & Modalité & Axe 1 & Axe 2 & $\mathbf{N}$ \\
\hline \multirow{13}{*}{ 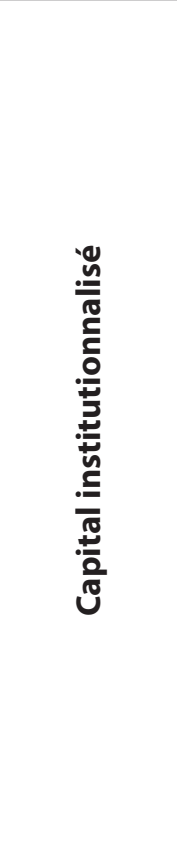 } & \multirow{7}{*}{$\begin{array}{l}\text { Orientation } \\
\text { de la formation } \\
\text { après le diplôme } \\
\text { de base }\end{array}$} & Orient: Médical ++ & 5,0 & 2,0 & 578 \\
\hline & & Orient: Médical + & 0,0 & 0,6 & 437 \\
\hline & & $\begin{array}{l}\text { Orient: Recherche } \\
\text { et Enseignement }\end{array}$ & 1,6 & 1,3 & 273 \\
\hline & & $\begin{array}{l}\text { Orient: Management } \\
\text { et Encadrement }\end{array}$ & 2,1 & 2,3 & 387 \\
\hline & & $\begin{array}{l}\text { Orient: Paramédical, } \\
\text { Développement } \\
\text { personnel et Religieux }\end{array}$ & 0,8 & 4,9 & 217 \\
\hline & & $\begin{array}{l}\text { Orient: Psychologie } \\
\text { et Social }\end{array}$ & 3,0 & 0,3 & 456 \\
\hline & & Total & 12,4 & 11,4 & 2348 \\
\hline & \multirow{5}{*}{$\begin{array}{l}\text { Niveau de la formation } \\
\text { après le diplôme } \\
\text { de base }\end{array}$} & Niveau: base & 3,1 & 0,7 & 527 \\
\hline & & Niveau: court & 0,1 & 3,4 & 534 \\
\hline & & Niveau: long & 0,4 & 2,6 & 1585 \\
\hline & & Niveau: académique & 3,3 & 0,1 & 190 \\
\hline & & Total & 6,9 & 6,9 & 2836 \\
\hline & Total & & 19,3 & 18,3 & -- \\
\hline \multirow{4}{*}{ 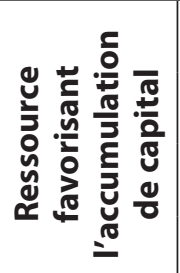 } & \multirow[t]{4}{*}{ Taux d'activité } & $0-60 \%$ & 0,8 & 4,3 & 691 \\
\hline & & $61-80 \%$ & 0,0 & 0,1 & 928 \\
\hline & & $81-100 \%$ & 0,5 & 3,2 & 1295 \\
\hline & & Total & 1,3 & 7,6 & 2914 \\
\hline
\end{tabular}

Note: En accord avec les recommandations de Brigitte Le Roux et Henry RouAnet (2010), toutes les modalités des variables actives contiennent au minimum $5 \%$ de l'échantillon. Seules les modalités dont les contributions sont égales ou supérieures à la contribution moyenne $(100 / 46=2,17)$ sont retenues pour l'interprétation des axes. Elles figurent en gras dans le tableau. 
Tableau A12. Variables et modalités actives caractéristiques des fractions

\begin{tabular}{|c|c|c|c|c|c|c|c|}
\hline & Variable & Modalité & \begin{tabular}{|c|} 
Dominantes \\
médicales \\
$n=654$ \\
$(22,37 \%)$ \\
\end{tabular} & $\begin{array}{c}\text { Dominées } \\
\text { médicales } \\
n=922 \\
(31,54 \%)\end{array}$ & $\begin{array}{c}\text { Élites } \\
\text { infirmières } \\
n=667 \\
(22,82 \%)\end{array}$ & $\begin{array}{c}\text { Hétérodoxes } \\
n=680 \\
(23,26 \%)\end{array}$ & \\
\hline & & & \multicolumn{4}{|c|}{$\%$ colonne par fraction } & $\begin{array}{c}\% \\
\text { colonne } \\
\text { total }\end{array}$ \\
\hline \multirow{6}{*}{ 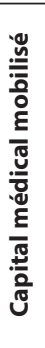 } & \multirow{3}{*}{\begin{tabular}{|l|} 
Mobilisation \\
des connaissances \\
en utilisation \\
de machines/ \\
appareils \\
techniques \\
\end{tabular}} & Rare Machine & 5,2 & 41,1 & 49,2 & 79,6 & 43,9 \\
\hline & & Souvent Machine & 17,6 & 48,1 & 23,6 & 17,5 & 28,6 \\
\hline & & Toujours Machine & 77,2 & 10,8 & 27,2 & 2,9 & 27,5 \\
\hline & Mobilisation & Rare Physio & 3,8 & 27,5 & 20,8 & 38,1 & 23,1 \\
\hline & $\begin{array}{l}\text { des connalssances } \\
\text { en physio- }\end{array}$ & Souvent Physio & 22,9 & 63,2 & 41,6 & 52,6 & 46,8 \\
\hline & pathologie & Toujours Physio & 73,3 & 9,3 & 37,6 & 9,3 & 30,1 \\
\hline \multirow{14}{*}{ 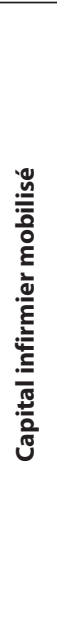 } & \multirow{3}{*}{$\begin{array}{l}\text { Mobilisation } \\
\text { des connaissances } \\
\text { en recherche }\end{array}$} & Rare Recherche & 52,5 & 77,8 & 20,4 & 47,9 & 52,1 \\
\hline & & Souvent Recherche & 35,3 & 21,1 & 40,2 & 43,8 & 33,9 \\
\hline & & Toujours Recherche & 12,3 & 1,1 & 39,4 & 8,3 & 14,0 \\
\hline & \multirow{3}{*}{\begin{tabular}{|l} 
Mobilisation \\
des valeurs \\
personnelles
\end{tabular}} & Rare Valeurs perso & 35,9 & 31,7 & 7,8 & 11,8 & 22,5 \\
\hline & & Souvent Val, perso & 37,2 & 55,4 & 20,9 & 45,2 & 41,1 \\
\hline & & Toujours Val, perso & 27,9 & 12,9 & 71,3 & 43,0 & 36,4 \\
\hline & \multirow{3}{*}{\begin{tabular}{|l|} 
Mobilisation \\
des connaissances \\
en modèles \\
théoriques \\
infirmiers \\
\end{tabular}} & Rare Modèles Inf & 50,6 & 59,1 & 18,7 & 38,9 & 43,3 \\
\hline & & Souvent Modèles Inf & 28,8 & 37,6 & 38,3 & 50,9 & 38,9 \\
\hline & & Toujours Modèles Inf & 20,6 & 3,3 & 43,0 & 10,2 & 17,8 \\
\hline & \multirow{2}{*}{\begin{tabular}{|l|} 
Mobilisation \\
des compétences \\
relationnelles
\end{tabular}} & Moins Relationnel & 31,0 & 44,5 & 5,6 & 15,1 & 25,8 \\
\hline & & Plus Relationnel & 69,0 & 55,5 & 94,4 & 84,9 & 74,2 \\
\hline & \multirow{3}{*}{$\begin{array}{l}\text { Mobilisation } \\
\text { de l'intuition }\end{array}$} & Rare Intuition & 24,6 & 33,5 & 10,4 & 13,9 & 21,6 \\
\hline & & Souvent Intuition & 49,5 & 54,6 & 37,5 & 51,5 & 48,9 \\
\hline & & Toujours Intuition & 25,9 & 11,9 & 52,1 & 34,6 & 29,5 \\
\hline
\end{tabular}




\begin{tabular}{|c|c|c|c|c|c|c|c|}
\hline & Variable & Modalité & \begin{tabular}{|c|} 
Dominantes \\
médicales \\
$n=654$ \\
$(22,37 \%)$ \\
\end{tabular} & $\begin{array}{c}\text { Dominées } \\
\text { médicales } \\
n=922 \\
(31,54 \%)\end{array}$ & $\begin{array}{c}\text { Élites } \\
\text { infirmières } \\
n=667 \\
(22,82 \%)\end{array}$ & $\begin{array}{c}\text { Hétérodoxes } \\
n=680 \\
(23,26 \%)\end{array}$ & \\
\hline & & & & $\%$ colonne & par fraction & & $\begin{array}{c}\% \\
\text { colonne } \\
\text { total }\end{array}$ \\
\hline \multirow{12}{*}{ 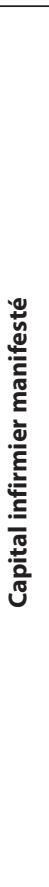 } & \multirow{3}{*}{$\begin{array}{l}\text { Conseiller } \\
\text { aux patients } \\
\text { d'avoir recours } \\
\text { à de la médecine } \\
\text { complémentaire }\end{array}$} & $\begin{array}{l}\text { Jamais ConseilMéd } \\
\text { Compl }\end{array}$ & 68,9 & 37,2 & 28,3 & 10,7 & 37,7 \\
\hline & & $\begin{array}{l}\text { Parfois ConseilMéd } \\
\text { Compl }\end{array}$ & 30,8 & 60,0 & 65,7 & 65,3 & 54,8 \\
\hline & & $\begin{array}{l}\text { Souvent ConseilMéd } \\
\text { Compl }\end{array}$ & 0,3 & 2,8 & 6,0 & 24,0 & 7,5 \\
\hline & \multirow{2}{*}{ Affiliation à I'ASI } & ASI: Non & 83,2 & 79,7 & 58,0 & 41,8 & 66,7 \\
\hline & & ASI: Oui & 16,8 & 20,3 & 42,0 & 58,2 & 33,3 \\
\hline & \multirow{2}{*}{$\begin{array}{l}\text { Congrès } \\
\text { de développement } \\
\text { personnel }\end{array}$} & $\begin{array}{l}\text { Non CongrèsDév } \\
\text { Perso }\end{array}$ & 97,4 & 94,0 & 84,6 & 67,9 & 86,6 \\
\hline & & $\begin{array}{l}\text { Oui CongrèsDév } \\
\text { Perso }\end{array}$ & 2,6 & 6,0 & 15,4 & 32,1 & 13,4 \\
\hline & \multirow{2}{*}{$\begin{array}{l}\text { Congrès } \\
\text { de médecine } \\
\text { complémentaire }\end{array}$} & $\begin{array}{l}\text { Non CongrèsMéd } \\
\text { Comp }\end{array}$ & 99,7 & 97,4 & 96,4 & 84,0 & 94,6 \\
\hline & & $\begin{array}{l}\text { Oui CongrèsMéd } \\
\text { Comp }\end{array}$ & 0,3 & 2,6 & 3,6 & 16,0 & 5,4 \\
\hline & \multirow{2}{*}{ Congrès de soins } & Non CongrèsSoins & 65,9 & 71,5 & 40,5 & 53,7 & 59,0 \\
\hline & & Oui CongrèsSoins & 34,1 & 28,5 & 59,5 & 46,3 & 41,0 \\
\hline & $\begin{array}{l}\text { Proposer aux } \\
\text { patients des soins } \\
\text { qui ne respectent } \\
\text { pas les consignes } \\
\text { de l'employeur et/ } \\
\text { ou hiérarchie }\end{array}$ & $\begin{array}{l}\text { Non SoinsContre } \\
\text { Hiérarchie } \\
\text { Oui SoinsContre } \\
\text { Hiérarchie }\end{array}$ & 86,0 & 69,3 & 62,7 & 54,7 & 33,0 \\
\hline
\end{tabular}




\begin{tabular}{|c|c|c|c|c|c|c|c|}
\hline & Variable & Modalité & $\begin{array}{c}\text { Dominantes } \\
\text { médicales } \\
n=654 \\
(22,37 \%)\end{array}$ & $\begin{array}{c}\text { Dominées } \\
\text { médicales } \\
n=922 \\
(31,54 \%)\end{array}$ & $\begin{array}{c}\text { Élites } \\
\text { infirmières } \\
n=667 \\
(22,82 \%)\end{array}$ & $\begin{array}{c}\text { Hétérodoxes } \\
n=680 \\
(23,26 \%)\end{array}$ & \\
\hline & & & & $\%$ colonne & par fraction & & $\begin{array}{c}\% \\
\text { colonne } \\
\text { total }\end{array}$ \\
\hline \multirow{10}{*}{ 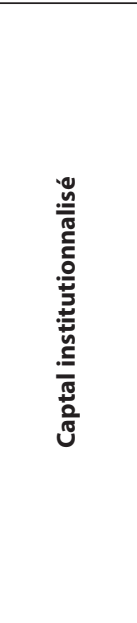 } & \multirow[t]{6}{*}{$\begin{array}{l}\text { Orientation } \\
\text { de la formation }\end{array}$} & Orient: Médical ++ & 65,5 & 25,4 & 11,7 & 4,5 & 24,6 \\
\hline & & Orient: Médical + & 15,5 & 30,5 & 11,5 & 16,3 & 18,6 \\
\hline & & $\begin{array}{l}\text { Orient: Recherche } \\
\text { et Enseignement }\end{array}$ & 5,9 & 5,8 & 21,7 & 12,0 & 11,7 \\
\hline & & $\begin{array}{l}\text { Orient: Management } \\
\text { et Encadrement }\end{array}$ & 6,6 & 7,3 & 35,6 & 14,7 & 16,5 \\
\hline & & \begin{tabular}{|l|} 
Orient: Paramédical, \\
Développement \\
personnel et Religieux
\end{tabular} & 1,4 & 14,7 & 1,6 & 17,4 & 9,2 \\
\hline & & $\begin{array}{l}\text { Orient: Psychologie } \\
\text { et Social }\end{array}$ & 5,1 & 16,3 & 17,9 & 35,1 & 19,4 \\
\hline & \multirow{4}{*}{\begin{tabular}{|l|} 
Niveau \\
de la formation
\end{tabular}} & Niveau: base & 24,3 & 32,3 & 6,6 & 6,4 & 18,6 \\
\hline & & Niveau: court & 15,7 & 29,0 & 7,3 & 19,4 & 18,8 \\
\hline & & Niveau: long & 59,2 & 36,9 & 74,3 & 60,1 & 55,9 \\
\hline & & Niveau: académique & 0,8 & 1,8 & 11,8 & 14,1 & 6,7 \\
\hline \multirow{3}{*}{ 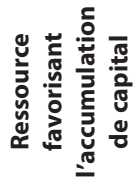 } & \multirow[t]{3}{*}{ Taux d'activité } & $0-60 \%$ & 10,4 & 28,6 & 12,8 & 40,6 & 23,7 \\
\hline & & $61-80 \%$ & 27,0 & 36,8 & 28,4 & 33,1 & 31,8 \\
\hline & & $81-100 \%$ & 62,6 & 34,6 & 58,8 & 26,3 & 44,5 \\
\hline
\end{tabular}

Note: En gras figurent les modalités dont la valeur test est égale ou supérieure à 2 en valeur absolue. 
Tableau A13. Variables et modalités supplémentaires caractéristiques des fractions

\begin{tabular}{|c|c|c|c|c|c|c|c|}
\hline & Variable & Modalité & $\begin{array}{c}\text { Dominantes } \\
\text { médicales } \\
n=654 \\
(22,37 \%)\end{array}$ & $\begin{array}{c}\text { Dominées } \\
\text { médicales } \\
n=922 \\
(31,54 \%)\end{array}$ & $\begin{array}{c}\text { Élites } \\
\text { infirmières } \\
n=667 \\
(22,82 \%)\end{array}$ & \begin{tabular}{|c|} 
Hétérodoxes \\
$n=680$ \\
$(23,26 \%)$
\end{tabular} & \\
\hline & & & \multicolumn{4}{|c|}{$\%$ colonne par fraction } & $\begin{array}{c}\% \text { colonne } \\
\text { total }\end{array}$ \\
\hline \multirow{15}{*}{ 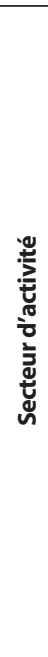 } & \multirow[t]{15}{*}{ Secteur } & Soins intensifs & 17,4 & 3,4 & 3,0 & 0,3 & 5,7 \\
\hline & & Bloc opératoire & 9,8 & 0,9 & 1,9 & 0,1 & 2,9 \\
\hline & & Urgences & 9,3 & 5,5 & 3,3 & 1,0 & 4,8 \\
\hline & & Soins continus & 5,2 & 2,6 & 1,2 & 0,6 & 2,4 \\
\hline & & Pédiatrie & 13,0 & 12,7 & 6,0 & 4,6 & 9,3 \\
\hline & & Chirurgie & 9,6 & 11,4 & 7,3 & 3,8 & 8,3 \\
\hline & & Rééducation & 3,5 & 5,4 & 4,2 & 2,2 & 4,0 \\
\hline & & Ambulatoire & 2,4 & 4,4 & 2,5 & 2,8 & 3,2 \\
\hline & & Médecine & 6,3 & 7,5 & 5,4 & 3,7 & 5,9 \\
\hline & & $\begin{array}{l}\text { Enseignement } \\
\text { et recherche }\end{array}$ & 0,6 & 0,7 & 10,5 & 5,3 & 4,0 \\
\hline & & Psychiatrie & 2,0 & 6,7 & 10,5 & 12,4 & 7,8 \\
\hline & & Santé scolaire & 0,3 & 2,1 & 3,3 & 13,5 & 4,6 \\
\hline & & Soins à domicile & 1,2 & 4,1 & 7,5 & 11,8 & 6,0 \\
\hline & & $\begin{array}{l}\text { Établissement } \\
\text { socio-éducatif }\end{array}$ & 0,5 & 1,7 & 1,3 & 5,1 & 2,2 \\
\hline & & EMS & 1,7 & 4,6 & 6,4 & 9,4 & 5,5 \\
\hline \multirow{7}{*}{ 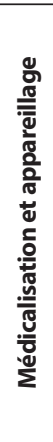 } & \begin{tabular}{|l|} 
Indice \\
de médicalisation
\end{tabular} & - & & & 31,8 & 50,2 & 25,5 \\
\hline & $\begin{array}{l}\text { (fréquence } \\
\text { des contacts directs }\end{array}$ & - & 16,7 & 30,0 & 27,2 & 26,0 & 25,4 \\
\hline & $\begin{array}{l}\text { ou indirects } \\
\text { entre infirmières }\end{array}$ & + & 33,5 & 33,1 & 20,8 & 17,2 & 26,7 \\
\hline & et médecins) & ++ & 44,4 & 19,6 & 20,2 & 6,6 & 22,4 \\
\hline & \begin{tabular}{|l|} 
Indice \\
d'appareillage
\end{tabular} & -- & 8,4 & 21,5 & 41,0 & 62,1 & 30,6 \\
\hline & & +- & 28,8 & 49,9 & 36,4 & 31,0 & 38,1 \\
\hline & $\begin{array}{l}\text { d'une liste } \\
\text { de } 8 \text { appareils) }\end{array}$ & ++ & 62,8 & 28,6 & 22,6 & 6,9 & 31,3 \\
\hline \multirow{6}{*}{ 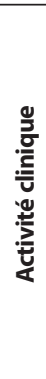 } & \multirow{2}{*}{\begin{tabular}{|l} 
Sans vs, \\
avec patients
\end{tabular}} & Sans & 3,2 & 3,5 & 35,2 & 16,3 & 13,6 \\
\hline & & Avec & 96,8 & 96,5 & 64,8 & 83,7 & 86,4 \\
\hline & \begin{tabular}{|l|} 
Indice de soins \\
de confort \\
et d'hygiène
\end{tabular} & -- & 8,7 & 18,5 & 29,5 & 48,2 & 24,7 \\
\hline & $\begin{array}{l}\text { (fréquence } \\
\text { de dispensation }\end{array}$ & - & 25,8 & 23,9 & 28,2 & 23,8 & 25,1 \\
\hline & $\begin{array}{l}\text { de } 8 \text { types de soins } \\
\text { impliquant }\end{array}$ & + & 33,8 & 29,8 & 18,1 & 14,0 & 25,2 \\
\hline & $\begin{array}{l}\text { une manipulation } \\
\text { du corps du patient) }\end{array}$ & ++ & 31,7 & 27,8 & 24,2 & 14,0 & 25,0 \\
\hline
\end{tabular}




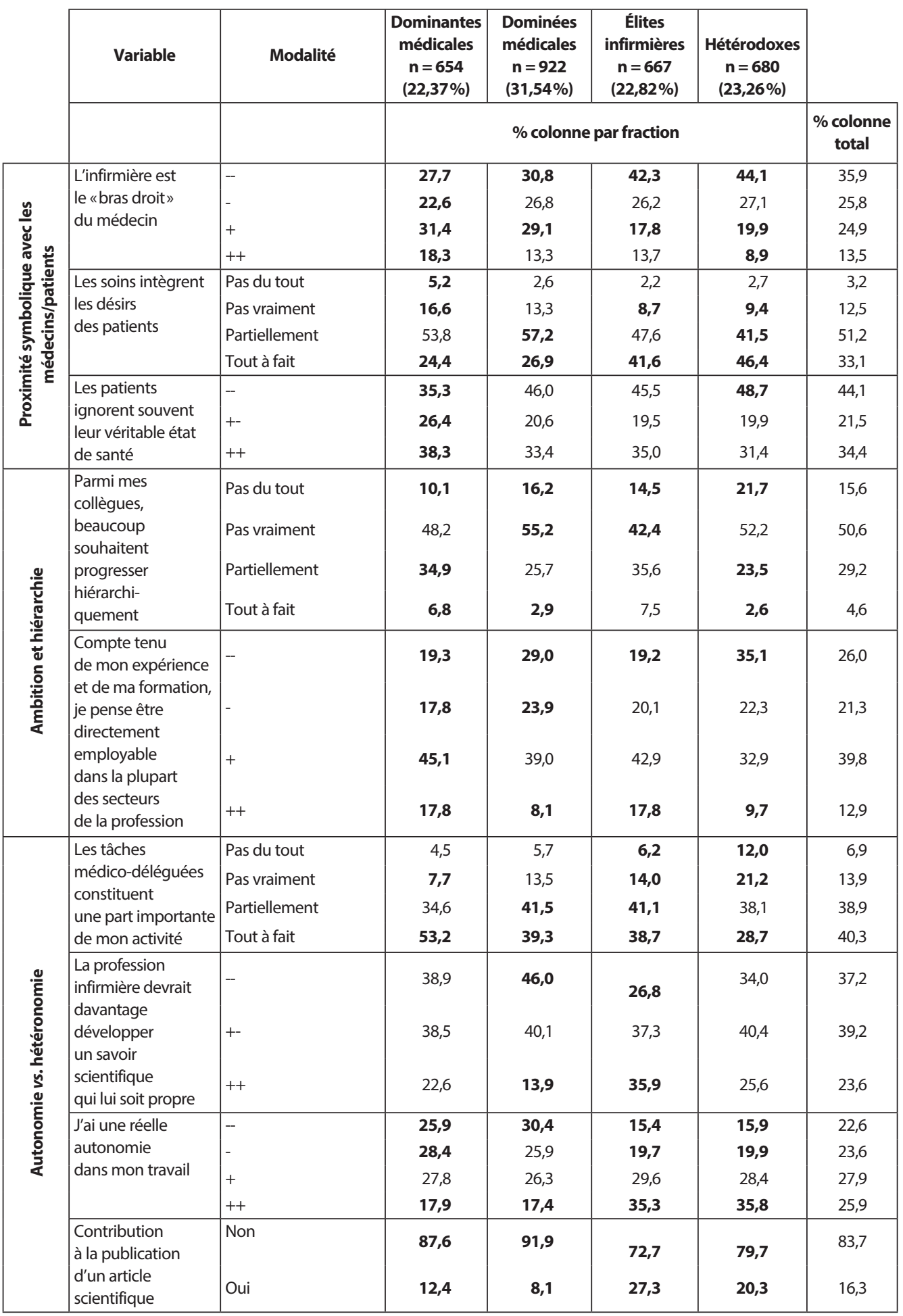




\begin{tabular}{|c|c|c|c|c|c|c|c|}
\hline & Variable & Modalité & \begin{tabular}{|c|} 
Dominantes \\
médicales \\
$n=654$ \\
$(22,37 \%)$ \\
\end{tabular} & $\begin{array}{c}\text { Dominées } \\
\text { médicales } \\
n=922 \\
(31,54 \%)\end{array}$ & $\begin{array}{c}\text { Élites } \\
\text { infirmières } \\
n=667 \\
(22,82 \%)\end{array}$ & $\begin{array}{c}\text { Hétérodoxes } \\
n=680 \\
(23,26 \%)\end{array}$ & \\
\hline & & & \multicolumn{4}{|c|}{$\%$ colonne par fraction } & $\begin{array}{c}\% \text { colonne } \\
\text { total }\end{array}$ \\
\hline \multirow{8}{*}{ 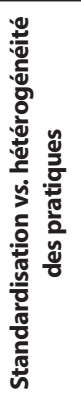 } & \multirow{4}{*}{\begin{tabular}{|l|} 
Chaque infirmière \\
a la possibilité \\
de développer \\
sa propre manière \\
de donner les soins \\
\end{tabular}} & Pas du tout & 16,9 & 12,4 & 9,8 & 5,6 & 11,6 \\
\hline & & Pas vraiment & 30,9 & 31,4 & 22,9 & 15,0 & 26,2 \\
\hline & & Partiellement & 42,5 & 46,0 & 48,2 & 54,9 & 47,4 \\
\hline & & Tout à fait & 9,7 & 10,2 & 19,1 & 24,5 & 14,8 \\
\hline & \begin{tabular}{|l|} 
Dans mon service/ \\
secteur d'activité,
\end{tabular} & & & & 14,0 & 7,8 & 17,2 \\
\hline & \begin{tabular}{|l|} 
I'expérience de vie \\
est aussi importante
\end{tabular} & - & 31,3 & 33,5 & 32,6 & 27,2 & 31,4 \\
\hline & que la formation & + & 32,5 & 36,6 & 35,2 & 44,3 & 37,1 \\
\hline & des soins & ++ & 8,7 & 10,9 & 18,2 & 20,7 & 14,3 \\
\hline \multirow{8}{*}{ 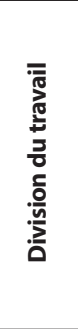 } & \multirow{4}{*}{\begin{tabular}{|l|} 
Je dois rappeler \\
aux autres \\
professionnels \\
quel est mon rôle
\end{tabular}} & Jamais & 30,2 & 34,3 & 25,3 & 21,5 & 28,9 \\
\hline & & Parfois & 58,0 & 56,1 & 53,7 & 50,3 & 54,9 \\
\hline & & Souvent & 10,5 & 9,3 & 16,0 & 23,5 & 13,8 \\
\hline & & Toujours & 1,3 & 0,3 & 5,0 & 4,7 & 2,4 \\
\hline & \multirow{4}{*}{\begin{tabular}{|l} 
Je dois effectuer \\
des tâches \\
pour lesquelles \\
je m'estime \\
surqualifiée
\end{tabular}} & Jamais & 21,1 & 25,1 & 18,0 & 26,3 & 22,8 \\
\hline & & Parfois & 42,4 & 46,0 & 44,7 & 50,7 & 46,0 \\
\hline & & Souvent & 31,7 & 26,2 & 32,5 & 21,7 & 27,8 \\
\hline & & Toujours & 4,8 & 2,7 & 4,8 & 1,3 & 3,4 \\
\hline \multirow{2}{*}{ 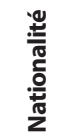 } & \multirow[t]{2}{*}{ Suisse vs. Étranger } & Suisse & 49,3 & 57,9 & 62,2 & 78,4 & 61,7 \\
\hline & & Étranger & 50,7 & 42,1 & 37,8 & 21,6 & 38,3 \\
\hline
\end{tabular}

Note: En gras figurent les modalités dont la valeur test est égale ou supérieure à 2 en valeur absolue. Les secteurs dont la valeur test est inférieure à 2 pour toutes les fractions ne figurent pas dans le tableau. 


\section{Table des matières}

Introduction .......................................................

Partie I. Une Profession homogène? ........................... 15

Chapitre 1. Éléments d'histoire

de la profession infirmière ............................................ 17

De la profession de foi à la profession ........................ 17

Le mythe de la «femme soignante» ........................... 18

Au service de l'Église ................................................. 19

L'avènement d'une professionnelle des soins ................ 21

Un processus de laïcisation en demi-teinte ................... 22

De la domination religieuse

à la domination médicale.......................................... 24

L'avènement d'une discipline infirmière:

un pas de plus vers l'autonomie de la profession ?.......... 24

Chapitre 2. Quelques éléments descriptifs de la profession...... 27

Genre, âge et formation.......................................... 27

Une force de travail étrangère.................................... 29

Le revenu............................................................. 31

L'origine sociale................................................... 36 
Chapitre 3. L'espace infirmier.

L'espace infirmier et le champ médical:

un rapport de domination

Deux formes de capital efficientes

au sein de l'espace infirmier

L'espace infirmier

Un espace de positions

Chapitre 4. Le prestige des secteurs d'activité

Le prestige des secteurs infirmiers

selon certaines caractéristiques classiques

Le prestige des secteurs infirmiers selon certaines

caractéristiques spécifiques

L'échelle de prestige: un consensus absolu entre les infirmières

Partie II. Visions ET Divisions

Chapitre 5. Les dominantes médicales

Des secteurs de référence .......................................... 66

La volatilité du capital médical ................................. 69

Une relative indifférenciation des tâches entre infirmières et médecins....................................... 71

Une autonomie spécifique ........................................ 74

Une distance avec les subalternes et les patients............ 76 Encadré 1 : Sandrine Jacques, une «dominante médicale».

Chapitre 6. Les dominées médicales............................... 87

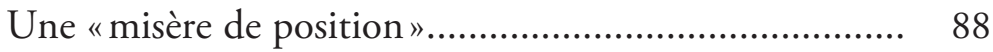

Une position d'intermédiaire entre patients et médecins ........................................ 90

Chapitre 7. Les élites infirmières................................... 93

En lutte pour une autodétermination professionnelle.... 94 
Les infirmières titulaires d'un master:

entre émancipation et tensions professionnelles ............ 97

Encadré 2: Claire Fabre, une «élite infirmière»............ 102

Chapitre 8. Les hétérodoxes ............................................ 109

Capital importé et mises en œuvre clandestines............ 110

Une hétérogénéité des pratiques ................................ 113

Une proximité avec les subalternes et les patients .......... 114

Encadré 3: Monique Emery, une «hétérodoxe» ........... 118

Chapitre 9. La dynamique de l'espace infirmier.............. 125

Un mouvement général des secteurs hospitaliers vers les secteurs extrahospitaliers ............................... 129

Une dissociation entre trajectoires professionnelles et trajectoires sociales................................................. 132

Partie III. Au Ceeur de l'ethos Professionnel................ 139

Chapitre 10. Les motivations sous-jacentes au choix professionnel .............................................. 141

L'influence des caractéristiques sociales sur les motivations .................................................. 145

L'influence des motivations sur les positions professionnelles

Chapitre 11. Une profession comme les autres?

Vision désenchantée et origine de classe........................ 153

Un processus de normalisation largement entamé ......... 154

Désenchantement et origine sociale........................... 155

Une «avant-garde désenchantée »............................... 157

Encadré 4: Déclassement culturel

et désenchantement. Le cas de Madame Maceira.......... 161

Chapitre 12. "Technique et relationnel»:

les dessous d'une doxa professionnelle............................. 171 
Humanisation des soins et valorisation

de la dimension « relationnelle " .................................. 172

Le couple «relationnel/technique»

comme principe de classement et de déclassement:

un dénigrement en cascade........................................ 175

Relationnel expressif et relationnel instrumental........... 178

Conclusion ........................................................... 183

Un espace infirmier diversifié, inégalitaire

et dynamique........................................................ 184

Généralisation des résultats et enjeux pour l'avenir de la profession..................................... 187

Tout ça pour ça ? De quelques réflexions

sur la réception de ce livre ........................................ 191

Postface de Patrice Pinell .............................................. 197

Remerciements ....................................................... 207

Bibliographie .......................................................... 209

Annexes ................................................................. 221

Annexe 1. L'enquête................................................. 221

Annexe 2. De la théorie à la mesure.

Opérationnalisation des capitaux médical et infirmier ............................................................. 225

Annexe 3. Tableaux..................................................... 230

Annexe 4. L'espace infirmier ..................................... 245 

Achevé d'imprimer

en avril 2020

Pour le compte des Éditions Livreo-Alphil

Responsable de production: Anne-Caroline Le Coultre 
J'ai jamais voulu être infirmière! Je voulais pas avoir une blouse, être dans un hôpital, faire des prises de sang...

Rien ne semblait prédisposer Madame Maceira à devenir infirmière. Voilà pourtant 15 ans qu'elle exerce cette profession avec passion en psychiatrie. Mais sans blouse, sans faire de prises de sang, et loin de l'hôpital. Elle n'est donc pas une infirmière comme les autres.

Mais qui sont donc ces autres infirmières? Existe-t-il une infirmière type? Est-ce nécessairement une femme qui, touchée par une vocation précoce, exerce dans un hôpital au chevet des patients?

En répondant à ces questions, les auteurs dressent un portrait totalement inédit de la profession qui rompt avec son apparente homogénéité. S'appuyant sur plusieurs dizaines d'entretiens et près de 3000 questionnaires, ils révèlent l'existence d'un espace infirmier fortement différencié et hiérarchisé, dans lequel les différents points de vue professionnels sont étroitement liés aux positions occupées.

À l'heure où la profession se trouve confrontée à des enjeux majeurs (pénurie de personnel, vieillissement de la population, rationalisation des soins, académisation des formations et développement de la recherche), ce livre constitue un outil de compréhension précieux pour tous les professionnels de santé et les étudiants. En mobilisant la théorie des champs de Pierre Bourdieu, il contribue plus largement au renouvellement de l'analyse sociologique des professions.

\section{Postface de Patrice Pinell}

Philippe Longchamp est professeur de sociologie à la Haute École de Santé Vaud (HESAV/HES-SO). Ses travaux portent sur la profession infirmière, les relations entre les professionnels de santé et leurs publics, les enjeux sociaux du corps et les inégalités sociales de santé.

Kevin Toffel est sociologue, enseignant-chercheur à la Haute École de Santé Vaud (HESAV/HES-SO). À la croisée des sociologies des professions, du corps et de la sexualité, ses travaux portent en particulier sur les rapports sociaux dans les professions de santé, ainsi que sur les questions que pose la sexualité dans les soins.

Felix Bühlmann est professeur associé en sociologie à l'Université de Lausanne. Ses intérêts de recherche portent sur les parcours de vie, la sociologie politique et la sociologie économique.

Amal Tawfik est sociologue, enseignant-chercheur à la Haute École de Santé Vaud (HESAV/HES-SO). Spécialiste des méthodes quantitatives, ses intérêts de recherche portent sur les inégalités sociales, les professions de santé, les parcours de vie et le comportement politique. 